\title{
Front propagation into unstable states: universal algebraic convergence towards uniformly translating pulled fronts
}

\author{
Ute Ebert $^{\mathrm{a}, \mathrm{b}, *}$, Wim van Saarloos ${ }^{\mathrm{a}}$ \\ a Instituut-Lorentz, Universiteit Leiden, Postbus 9506, 2300 RA Leiden, Netherlands \\ ${ }^{\mathrm{b}}$ CWI, Postbus 94079, 1090 GB Amsterdam, Netherlands \\ Received 1 April 1999; received in revised form 1 February 2000; accepted 14 March 2000 \\ Communicated by A.C. Newell
}

\begin{abstract}
Fronts that start from a local perturbation and propagate into a linearly unstable state come in two classes: pulled fronts and pushed fronts. The term "pulled front" expresses that these fronts are "pulled along" by the spreading of linear perturbations about the unstable state. Accordingly, their asymptotic speed $v^{*}$ equals the spreading speed of perturbations whose dynamics is governed by the equations linearized about the unstable state. The central result of this paper is the analysis of the convergence of asymptotically uniformly traveling pulled fronts towards $v^{*}$. We show that when such fronts evolve from "sufficiently steep" initial conditions, which initially decay faster than $\mathrm{e}^{-\lambda^{*} x}$ for $x \rightarrow \infty$, they have a universal relaxation behavior as time $t \rightarrow \infty$ : the velocity of a pulled front always relaxes algebraically like $v(t)=v^{*}-3 /\left(2 \lambda^{*} t\right)+\frac{3}{2} \sqrt{\pi} D \lambda^{*} /\left(D \lambda^{*} t\right)^{3 / 2}+\mathrm{O}\left(1 / t^{2}\right)$. The parameters $v^{*}, \lambda^{*}$, and $D$ are determined through a saddle point analysis from the equation of motion linearized about the unstable invaded state. This front velocity is independent of the precise value of the front amplitude, which one tracks to measure the front position. The interior of the front is essentially slaved to the leading edge, and develops universally as $\phi(x, t)=\Phi_{v(t)}\left(x-\int^{t} \mathrm{~d} t^{\prime} v\left(t^{\prime}\right)\right)+\mathrm{O}\left(1 / t^{2}\right)$, where $\Phi_{v}(x-v t)$ is a uniformly translating front solution with velocity $v<v^{*}$. Our result, which can be viewed as a general center manifold result for pulled front propagation is derived in detail for the well-known nonlinear diffusion equation of type $\partial_{t} \phi=\partial_{x}^{2} \phi+\phi-\phi^{3}$, where the invaded unstable state is $\phi=0$. Even for this simple case, the subdominant $t^{-3 / 2}$ term extends an earlier result of Bramson. Our analysis is then generalized to more general (sets of) partial differential equations with higher spatial or temporal derivatives, to PDEs with memory kernels, and also to difference equations such as those that occur in numerical finite difference codes. Our universal result for pulled fronts thus implies independence (i) of the level curve which is used to track the front position, (ii) of the precise nonlinearities, (iii) of the precise form of the linear operators in the dynamical equation, and (iv) of the precise initial conditions, as long as they are sufficiently steep. The only remainders of the explicit form of the dynamical equation are the nonlinear solutions $\Phi_{v}$ and the three saddle point parameters $v^{*}, \lambda^{*}$, and $D$. As our simulations confirm all our analytical predictions in every detail, it can be concluded that we have a complete analytical understanding of the propagation mechanism and relaxation behavior of pulled fronts, if they are uniformly translating for $t \rightarrow \infty$. An immediate consequence of the slow algebraic relaxation is that the standard moving boundary approximation breaks down for weakly curved pulled fronts in two or three dimensions. In addition to our main result for pulled fronts, we also discuss the propagation and convergence of fronts emerging from
\end{abstract}

\footnotetext{
${ }^{*}$ Corresponding author. Present address: CWI, Postbus 94079, 1090 GB Amsterdam, Netherlands
} 
initial conditions which are not steep, as well as of pushed fronts. The latter relax exponentially fast to their asymptotic speed. (c) 2000 Elsevier Science B.V. All rights reserved.

Keywords: Pulled and pushed fronts; Universal relaxation behavior; Unstable states; Algebraic convergence

\section{Introduction}

\subsection{Outline of the problem}

In this paper (see Scheme 1), we address the rate of convergence or "relaxation" of the velocity and profile of a front that propagates into an unstable state. The particular fronts that we analyze separate two non-equilibrium homogeneous states, one of which is stable and one of which is unstable, and are such that the asymptotic front solution is a uniformly translating one. We assume that the unstable state is initially completely unperturbed in a large part of space, and that thermal and other noise are negligible. Examples of such situations arise in one form or another in physics [1-29,110-113,120], chemistry [29-35], ${ }^{1}$ and biology $[30,32,36,114]$. If the unstable state domain is not perturbed by imperfect initial conditions or thermal noise, it can only disappear through invasion by the stable state domain. We analyze the propagation of fronts formed in this process, in particular the temporal convergence towards an asymptotic front shape and velocity, and show that it is characterized by a universal power law behavior in the so-called pulled regime. We concentrate on planar fronts, which thus can be represented in one spatial dimension. However, our results for these and for the dynamical mechanism also have important implications [37] for the derivation of moving boundary approximations [38,39] for weakly curved fronts in higher dimensions, as well as for the evaluation of the effects of noise on fronts [40-46], especially the effect of multiplicative noise $[47,48]$.

The problem of front propagation into an unstable state has a long history, which dates back ${ }^{2}$ to the pioneering work by Kolmogoroff, Petrovsky and Piscounoff (KPP) [49] and by Fisher [50] on the nonlinear diffusion equation

$$
\partial_{t} \phi=\partial_{x}^{2} \phi+f(\phi)
$$

where $f(\phi)$ is such that it has a homogeneous stable state $\phi=1$ and a homogeneous unstable state $\phi=0$. The early work on this equation $[49,50]$ was motivated by the biological problem of gene spreading in a population. Since this work, the nonlinear diffusion equation (1.1), in particular the one with a simple nonlinearity of the type

$$
f=f_{\mathrm{KPP}}(\phi)=\phi-\phi^{k}, \quad k>1, \text { e.g., } k=2 \text { or } 3,
$$

has become a standard problem in the mathematical literature $[30,32,36,51-53,114] .3,4$ For the F-KPP equation defined by (1.1) and (1.2), there exist dynamically stable uniformly translating front solutions $\phi(x, t) \equiv \Phi_{v}(x-v t)$ for every velocity $v \geq v^{*}=2 f^{\prime}(0)^{1 / 2}$, and hence every one of these solutions is a possible attractor of the dynamics for long times $t$. The resulting dynamical behavior or "velocity selection" depends on the initial conditions and has been investigated by a variety of methods [49-51,53]; essentially all its relevant properties have been derived rigorously [51]. For example, following the lines of KPP [49], Aronson and Weinberger [51] proved rigorously that every initial condition, that decays spatially at least as fast as $\mathrm{e}^{-\lambda^{*} x}\left(\lambda^{*}=\frac{1}{2} v^{*}\right)$ into the unstable state for

\footnotetext{
${ }^{1}$ See especially the article by Shaul and Showalter in Ref. [31].

2 As mentioned by Murray [[36], p. 277], the equation was apparently already considered in 1906 by Luther, who obtained the same analytical form as Fisher for the wave front.

${ }^{3}$ For some recent more mathematical advances within the physics literature, see $[54,115]$.

${ }^{4}$ For a recent extension to multidimensional cases, and for an entry into the mathematical literature, see, e.g., [55].
} 
Table of content

\section{Introduction}

1.1 Outline of the problem

1.2 Pushed versus pulled fronts, selection and convergence

1.3 Sketch of method and results on front relaxation in the pulled regime

1.4 Organization of the paper

2 Stability, selection and convergence in the nonlinear diffusion equation

2.1 Statement of problem and essential concepts

2.2 Uniformly translating fronts: candidates for attractors and transients

2.3 Linear stability analysis of moving front solutions

2.4 Consequences of the stability analysis for selection and rate of convergence; marginal stability

2.5 The dynamics of the leading edge of a front

2.5.1 Equation linearized about $\phi=0$

2.5.2 Leading edge representation of the full equation

2.6 Concluding remarks - interior and edge dominated dynamics

3 Universal pulled convergence of steep initial conditions in the nonlinear diffusion equation

3.1 Observations which motivate our approach

3.1.1 Asymptotic steepness of leading edge determines rate of convergence

3.1.2 Interior follows leading edge: uniform convergence

3.1.3 Choose proper frame and subtraction for the interior

3.1.4 Choose proper expansions and match leading edge to interior

3.2 Expansion in the interior region

3.3 Interior shape expanded towards the leading edge

3.4 Analysis of the leading edge

3.5 Summarizing remarks

4 Simulations of pulled fronts in the nonlinear diffusion equation

4.1 Numerical features specific to pulled fronts

4.1.1 Effect of finite difference code

4.1.2 Effect of finite system size

4.2 Simulation data

4.2.1 $f(\phi)=\phi-\phi^{3}$ : pulled fronts

4.2.2 $f(\phi)=\epsilon \phi+\phi^{3}-\phi^{5}$ : pushed versus pulled fronts

4.3 Comparison of simulations and analytical predictions

4.3.1 Analysis of the velocity data

4.3.2 Analysis of the shape data

5 Generalization of pulling to higher order (sets of) equations

5.1 Introduction

5.2 Basic assumptions underlying the relaxation analysis of pulled fronts; generalization of Table 4

5.3 Pulled front relaxation in single p.d.e.'s of first order in time 5.3.1 The pulled velocity $v^{*}$ 
5.3.2 Uniformly translating solutions $\Phi_{v}$

5.3.3 The leading edge representation

5.3.4 The relaxation analysis

5.4 Generalization to single p.d.e.'s of higher order in time

5.5 Further generalizations

5.5.1 Long time asymptotics of the Green's function via a Fourier-Laplace transformation

5.5.2 The case of a single field

5.5.3 The case of a set of fields and possible projections

5.5.4 The freedom of projection and the universality of Tables 2 and 5

5.6 Applications

5.6.1 The EFK equation

5.6.2 The streamer equations

5.6.3 A difference-differential equation

5.6.4 Diffusion equation with second onder time derivative

5.6.5 An extension of the F-KPP equation with a memory kernel

5.6.6 Exact results for numerical finite difference schemes

6 Summary and outlook

6.1 Summary of the main results

6.2 Summary of the main conceptual steps of the analysis

6.3 Open problems

6.4 The multiplicity of front solutions and of solutions of the saddle point equations

6.5 A step by step guideline for applying these results

6.6 The subtle role of the nonlinearities: an alternative intuitive explanation

\section{APPENDICES}

A An upper bound for $v_{c}$ in the nonlinear diffusion equation

$B$ The generalized nonlinear diffusion equation

C Analytical solutions for pushed nonlinear diffusion fronts and transition to pulling

D Linear stability analysis of moving front solutions D.1 Schrödinger stability analysis

D.2 Linear perturbations outside the Hilbert space

E Stability analysis, selection and rate of convergence E.1 Pushed regime: $v_{c}=v^{\dagger}$

E.2 Fronts into metastable states

E.3 Pulled regime: $v_{c}=v^{*}$

$F$ General integration of $g_{n / 2}^{s p}(z)$

G Algebraic convergence at the pushed/pulled transition

$H$ Multiplicity of fronts and linear eigenmodes for reflection symmetric equations of first order in time

I Strongly heteroclinic orbits and change of stability at $v^{\dagger}$

J Relation between the generalized diffusion constants $D_{n}$ and the dispersion relation

K Edge analysis of uniformly translating pulled fronts with $M=1$

$L$ Leading edge projections for coupled equations: an example

$\mathrm{M}$ Pinch point versus saddle point analysis 
$x \rightarrow \infty$, approaches for large times the front solution $\Phi_{v^{*}}\left(x-v^{*} t\right)$ with the smallest possible velocity $v^{*}$. Most of the rigorous mathematical methods, however, cannot be extended to higher order equations. ${ }^{5}$

In physics, the interest in front propagation into unstable states initially arose from a different angle. Since the late 1950s, the growth and advection of linear perturbations about a homogeneous unstable state has been analyzed through an asymptotic large time expansion of the Green's function of the linear equations [56-58]. Only $10-15$ years ago it become fully clear in the physics community $[59-69,116]$ that there was actually an empirical but deep connection between the rigorous results for the second order equations and some aspects of the more general and exact but non-rigorous results for the growth of linear perturbations. This has given rise to a number of reformulations and intuitive scenarios aimed at understanding the general front propagation problem into unstable states $[60,61,63,65-69,116]$.

Although our results bear on many of these approaches, our aim is not to introduce another intuitive or speculative scenario. Rather, we will introduce what we believe to be the first systematic analysis of the rate of convergence or "relaxation" of the front velocity and profile in the so-called "linear marginal stability" $[63,65]$ or "pulled" $[59,68,69]$ regime. In this regime, the asymptotic front velocity is simply the linear spreading speed determined by the Green's function of the linearized equations. Quite surprisingly, our analysis even yields a number of new and exact results for the celebrated nonlinear diffusion equation (1.1), but it applies equally well to (sets of) higher order partial differential equations that admit uniformly translating fronts, to difference equations or to integro-differential equations. We will discuss such equations in general, and then illustrate our results on the example equations from Table 1.

For all such equations, our results have a remarkable degree of simplicity and universality: pulled fronts always converge in time with universal power laws and prefactors that are independent of the precise form of the equations and independent of the precise initial conditions as long as they obey a certain steepness criterion. To be precise, for equations such that the dynamically selected asymptotic front is a uniformly translating pulled front, and for the so-called sufficiently steep initial conditions defined such that $\lim _{x \rightarrow \infty} \phi(x, 0) \mathrm{e}^{\lambda x}=0$ for some $\lambda>\lambda^{*}$, we derive that the asymptotic velocity convergence is given by the universal law

$$
v(t)=v^{*}+\dot{X}, \quad \dot{X}=-\frac{3}{2 \lambda^{*} t}\left(1-\left(\frac{\pi}{\left(\lambda^{*}\right)^{2} D t}\right)^{1 / 2}\right)+\mathrm{O}\left(\frac{1}{t^{2}}\right) .
$$

The velocity $v^{*}$, the inverse length $\lambda^{*}$ and the diffusion constant $D$ are in general obtained from a saddle point expansion [58] for the equation of motion linearized about the unstable state. In a frame moving with velocity $v^{*}$ the quickest growing mode $k^{*}$ is identified by the complex saddle point equation $\left.\partial_{k}\left[\omega(k)-v^{*} k\right]\right|_{k=k^{*}}=0$, where $\omega(k)$ is the dispersion relation of a Fourier mode $\exp \{-i \omega t+i k x\}$. In the more usual decomposition into real functions this implies that $[56,61,63,65]$

$$
\left.\frac{\partial \operatorname{Im} \omega}{\partial \operatorname{Im} k}\right|_{k^{*}}=v^{*},\left.\quad \frac{\partial \operatorname{Im} \omega}{\partial \operatorname{Re} k}\right|_{k^{*}}=0 .
$$

The speed of the frame is asymptotically the same as the speed of the front if

$$
\frac{\operatorname{Im} \omega\left(k^{*}\right)}{\operatorname{Im} k^{*}}=v^{*}
$$

For the uniformly translating fronts that we will analyze here, we have

$$
\operatorname{Im} k^{*} \equiv \lambda^{*}>0, \quad \operatorname{Re} k^{*}=0, \quad \operatorname{Re} \omega\left(k^{*}\right)=0,
$$

\footnotetext{
${ }^{5}$ For a discussion of the few mathematically precise results that are available for more complicated or higher order equations, we refer to the book by Collet and Eckmann [52].
} 
Table 1

Summary of the equations studied in detail in Section 5.6 as examples of the general validity of our results for higher order equations, coupled equations, difference equations, and equations with a kernel. All these equations have pulled front solutions whose asymptotic speed relaxes according to (1.3)

The EFK equation:

The streamer equations:

\section{A difference equation from kinetic theory:}

A second order extension of the F-KPP equation:

An equation with a memory kernel:

Finite difference versions of the F-KPP equation like:

$$
\partial_{t} \phi=-\gamma \partial_{x}^{4} \phi+\partial_{x}^{2} \phi+f(\phi) \text { with } 0<\gamma<1 / 12
$$

$$
\left\{\begin{array}{l}
\partial_{t} \sigma=D \partial_{x}^{2} \sigma+\partial_{x}(\sigma E)+\sigma|E| e^{-1 /|E|} \\
\partial_{t} E=-D \partial_{x} \sigma-\sigma E
\end{array}\right.
$$

$$
\partial_{t} C_{i}(t)=-C_{i}+C_{i-1}^{2}
$$

$$
\begin{gathered}
\tau_{2} \partial_{t}^{2} \phi+\partial_{t} \phi=\partial_{x}^{2} \phi+\phi-\phi^{3} \\
\partial_{t} \phi(x, t)=\partial_{x}^{2} \phi(x, t)+\int_{0}^{t} d t^{\prime} K\left(t-t^{\prime}\right) \phi\left(x, t^{\prime}\right)-\phi^{k}(x, t) \\
\frac{u_{j}(t+\Delta t)-u_{j}(t)}{\Delta t}=\frac{u_{j+1}(t)-2 u_{j}(t)+u_{j-1}(t)}{(\Delta x)^{2}} \\
+u_{j}(t)-u_{j}^{k}(t)
\end{gathered}
$$

and a real positive diffusion coefficient $D$

$$
D=\left.\frac{\mathrm{i} \partial^{2} \omega}{2 \partial k^{2}}\right|_{k^{*}}=\left.\frac{\partial^{2} \operatorname{Im} \omega}{2 \partial \lambda^{2}}\right|_{k^{*}} .
$$

While the velocity of a front is converging to its asymptotic value, so is the profile shape. Note that $v(t)(1.3)$ does not depend on the "height" $\phi=h$, which is being tracked. In fact, if we define the velocity $v_{\phi}$ of the fixed amplitude $\phi=h$ through $\phi\left(x+\int^{t} \mathrm{~d} \tau v_{\phi}(\tau), t\right)=h$, then up to order $1 / t^{2}$ the velocity $v_{\phi}(t)=v^{*}+\dot{X}$ is independent of the "height" $\phi=h$. Moreover, it is determined solely by properties of the equation linearized about the unstable state, as Eqs. (1.4)-(1.7) show. In this sense, we can indeed speak of pulling of the front by the leading edge of the front.

The above expression for $v(t)$ contains all the universal terms, since the next $1 / t^{2}$ term in the long time expansion does depend on initial conditions. The above analytic results for the universal velocity convergence as well as related ones for the relaxation of the front profile which are summarized in Table 2 and discussed in more detail below, are fully confirmed by extremely precise numerical simulations. Taken together, this study therefore yields the understanding of the pulled front mechanism that so many authors $[8,61,63-65,67-70]$ have sought.

In this paper, first the asymptotic long time behavior is worked out in detail and to high orders for the F-KPP equation (1.1) and (1.2) in two matched asymptotic expansions in $1 / \sqrt{t}$. Once we have laid out the structure of this expansion, it is clear that essentially the same matched expansions can be applied to other more complicated types of equations, provided that they admit a family of uniformly translating front solutions in the neighborhood of the asymptotic "pulled" velocity $v^{*}$. Moreover, the two lowest order equations in the $1 / \sqrt{t}$ expansion in the 
so-called leading edge region together with a boundary condition suffice to calculate the universal convergence. The structure of these equations is virtually independent of the precise form of the dynamical equation. For more general equations, we hence limit the discussion to the motivation and analysis of these two equations. Although we will give some discussion of the assumptions that underly the expansion (like the one that there is a nearby family of moving front solutions), a full analysis of these as well as of the extension to non-uniformly translating fronts, such as those arising in the EFK equation of Table 1 for $\gamma>\frac{1}{12}$, in the Swift-Hohenberg equation [71], or in the complex Ginzburg-Landau equation [66], will be left to future publications [72].$^{6,7}$

For Eqs. (1.1) and (1.2), we simply have $v^{*}=2, \lambda^{*}=D=1$. The first term in (1.3) then reduces to a well-known result of Bramson [74], who rigorously proved that the convergence to the asymptotic velocity $v^{*}$ is $v(t)=v^{*}-3 /\left(2 \lambda^{*} t\right)$ uniformly, i.e., independent of the amplitude $\phi$ whose position one tracks. The factor $\frac{3}{2}$ in this expression has often been considered puzzling, since the linear diffusion equation with localized initial conditions yields $v(t)=v^{*}-1 /\left(2 \lambda^{*} t\right)+\cdots$. In [65], it was argued that the factor $\frac{3}{2}$ in this result applies more generally to higher order equations as well, but a systematic analysis or an argument for why the convergence is uniform, was missing. Apart from this and a recent rederivation [70] of Bramson's result along lines similar in spirit to ours ${ }^{8}$ and a few papers similar in spirit to that of Bramson $[53,76,77]^{9}$, we are not aware of systematic calculations of the velocity and profile relaxation. Even for the convergence of the velocity in the celebrated nonlinear diffusion equation, our $1 / t^{3 / 2}$ term appears to be new.

From a different perspective, Powell et al. [67] also considered the convergence properties of pulled fronts. These authors studied the shapes of the front profiles in the nonlinear diffusion equation and argued that they relax along the family of unstable uniformly translating front solutions. Although they realized that the velocity relaxation was algebraic and from below, they did not seem to realize that the dominant $-3 /(2 t)$ velocity correction was known from earlier work $[65,74]$. As we shall see below when we will discuss the shape relaxation of fronts, our derivation is the first analytic derivation and confirmation of the picture of Powell et al., and identifies the connection with the velocity relaxation.

Our results are not only of interest in their own right, but they also have important implications. Since the asymptotic convergence towards the attractor $\Phi^{*}$ is algebraic in time, the attractor alone might not give sufficient information about the front after long but finite times, since algebraic convergence has no characteristic timescale. In particular, there is no time beyond which convergence can be neglected. Such slow convergence means that in many cases, experimentally as well as theoretically, one observes transients and not the asymptotic behavior. In fact, in the very first explicit experimental test of front propagation into unstable states in a pattern forming system [2], viz. Taylor-Couette flow, the initial discrepancy between theory and experiment was later shown to be related to the existence of slow transients [16]. The slow convergence is important for theoretical studies as well: it is a common experience (see, e.g., $[12,64,78])^{10}$ that when studying front propagation in the "pulled" regime numerically, the measured transient front velocity is often below $v^{*}$. This is so even though the asymptotic front speed can never be below $v^{*}$, because no slower attractor of the dynamics exists. This observation finds a natural explanation in our finding that the rate of convergence is always power law slow, and that the front speed is always approached from below.

\footnotetext{
${ }^{6} \mathrm{~A}$ new and simple proof that fronts in the Swift-Hohenberg equations are pulled, and a new mode expansion that leads to a generalization of (1.3) for pattern forming fronts which asymptotically are periodic in the comoving frame, such as those arising in the Swift-Hohenberg equation, will be given in a future publication by Spruijt et al. [99].

${ }^{7}$ The convergence towards fronts whose dynamics remains non-periodic in the comoving frame, such as those in the complex Ginzburg-Landau equation for some values of the parameters [12,62,66,116] is discussed in [73].

${ }^{8}$ The main focus of the work by Brunet and Derrida [70] is actually the correction to the asymptotic velocity if the function $f(\phi)$ has a cutoff $h$ such that $f_{h}(\phi)=0$ for $\phi<h$. The method the authors use to derive this, is actually closely related to the one they use to rederive Bramson's result, and to our approach. See in this connection also the recent paper by Kessler et al. [75].

${ }^{9}$ We thank F.M. Hekking for bringing Ref. [76] to our attention.

${ }^{10}$ See, e.g., Fig. 6 of [78] which is a full numerical study of the predictions of Ref. [9].
} 
A second important implication of the absence of an intrinsic timescale of the front convergence is the following. When we consider the propagation of such fronts in more than one dimension in which there is a coupling to another slow field (as, e.g., in the phase field models $[39,79]),{ }^{11}$ the front dynamics does not adiabatically decouple from the dynamics of the other field and from the evolution of the curvature and shape of the front itself. This implies that the standard moving boundary approximation $[38,39,81]$ (which actually rests on the assumption that the convergence on the "inner scale" is exponential) cannot be made. Though this is intuitively quite obvious from the power law behavior of the front convergence process, the connection between the convergence and the breakdown of a moving boundary approximation also emerges at a technical level: the divergence of the solvability integrals that emerge when deriving a moving boundary approximation turns out to be related to the continuity of the stability spectrum of pulled fronts [37]. The break down of the solvability analysis for perturbations of the asymptotic front in the pulled regime also has consequences for the evaluation of multiplicative noise in such equations $[37,48]$.

\subsection{Pushed versus pulled fronts, selection and convergence}

Let us return to the well understood nonlinear diffusion equation (1.1) and discuss to which nonlinearities $f(\phi)$ our prediction of algebraic convergence applies and why. If $f^{\prime}(0)<0$, the invaded state $\phi=0$ is linearly stable, and the construction of a uniformly translating front $\phi(x, t)=\Phi_{v}(x-v t)$ posses a nonlinear eigenvalue problem. The solution with the largest eigenvalue $v$ is the unique stable and dynamically relevant solution (unique up to a translation, of course). As is well known and discussed in Section 2, any initial front that separates the (meta)stable state $\phi=0$ at $x \rightarrow \infty$ from another stable state at $x \rightarrow-\infty$ will converge exponentially in time to this unique attractor $\Phi_{v}$. However, whenever $f^{\prime}(0)>0, \phi=0$ is unstable, there is not a unique asymptotic attractor $\Phi_{v}$, but a continuous spectrum of nonlinear eigenvalues $v$ which constitute the velocities of possible attractors $\Phi_{v}$. The existence of a continuum of attractors of the dynamics posses a so-called selection problem: from which initial conditions will the front dynamically approach which attractor? The attractor with the smallest velocity plays a special role, as its basin of attraction are all "sufficiently steep" initial conditions, as defined in Section 2 . It therefore will be referred to as the selected front solution.

When we concentrate on these "sufficiently steep" initial conditions and analyze the dependence on the nonlinearity $f$ in (1.1), the transition from exponential to algebraic convergence does not coincide with the transition from stability to instability of the invaded state $\phi=0$, but with the transition between two different mechanisms of front propagation into unstable states. Indeed, it is known (see also Section 2), that for $f^{\prime}(0)>0$, there are two different mechanisms for how the selected front $\Phi_{\text {sel }}$ and its speed $v_{\text {sel }}$ are determined. Either $\Phi_{\text {sel }}$ is found by constructing a so-called strongly heteroclinic orbit for $\Phi_{v}$ from the full nonlinear equation. This case is also known as Case II [61] or nonlinear marginal stability [63,65], or as pushing [59,68,69]. Or, the selected velocity $v_{\text {sel }}$ is determined by linearizing about the unstable state $\phi=0$, which case is known as Case I or linear marginal stability, or as pulling. We, henceforth, will use the terms "pushing" and "pulling" for the two different propagation mechanisms of a selected front evolving from steep initial conditions, since they, very literally, express the different dynamical mechanisms.

In a pushed front just like in a front propagating into a (meta)stable state, the dynamics is essentially determined in the nonlinear "interior part" of the front, where $\phi$ varies from close to $\phi=0$ to close to the stable state. The construction of the selected front as a strongly heteroclinic orbit in the pushed case continuously extends into the construction of the heteroclinic orbit of the unique attractor if the invaded state is (meta)stable $\left(f^{\prime}(0)<0\right)$. For both pushed fronts and fronts propagating into linearly stable states, the spectrum of linear perturbations is bounded away from zero, so that convergence towards the asymptotic front is exponential in time.

\footnotetext{
${ }^{11}$ An entry into the more mathematically oriented literature is the paper by Bates et al. [80].
} 
In a pulled front, the dynamics is quite different: as we shall see, it is determined essentially in the region linearized about the unstable state. We call this region the leading edge of the front. Eq. (1.1) is appropriate for analyzing the front interior. We will see in Section 2.4 , that a stability analysis performed in this representation is not able to capture the convergence of a steep initial condition towards a pulled front. Rather the substitution

$$
\psi=\phi \mathrm{e}^{\lambda^{*} \xi}, \quad \xi=x-v^{*} t,
$$

which we shall term the leading edge representation, transforms (1.1) into

$$
\begin{aligned}
& \partial_{t} \psi=\partial_{\xi}^{2} \psi+\bar{f}(\psi, \xi), \\
& \bar{f} \equiv \mathrm{e}^{\lambda^{*} \xi}\left[f\left(\psi \mathrm{e}^{-\lambda^{*} \xi}\right)-f^{\prime}(0) \psi \mathrm{e}^{-\lambda^{*} \xi}\right]=o\left(\psi^{2} \mathrm{e}^{-\lambda^{*} \xi}\right) .
\end{aligned}
$$

This equation will turn out to be appropriate for analyzing a leading edge dominated dynamics. Note that $\bar{f}$ is at least of order $\psi^{2}$ with an exponentially small coefficient as $\xi \rightarrow \infty$. For large $\xi$, the dynamics is purely diffusive. If the nonlinearity obeys $f(\phi)-f^{\prime}(0) \phi<0$ for all $\phi>0$, which is known as a sufficient criterion for pulling, the nonlinearity $\bar{f}$ is always negative. Then $\bar{f}$ purely damps the dynamics in the region of smaller $\xi$. The dynamics evolving under (1.9) is equivalent to simply linearizing (1.1) about the unstable state in the large $\xi$ region - there is only one subtle but important ingredient from the requirement that the dynamics in the linear region crosses over smoothly to the nonlinear front behavior at smaller $\xi$ that actually enters our leading edge analysis in the form of a boundary condition. In the leading edge representation (1.9), this is brought out by the presence of the sink-type term $\bar{f}$ which is non-zero in a localized region behind the leading edge. With this small caveat, ${ }^{12}$ we can conclude that the leading edge of the front "pulls the rest of the front along", which is precisely the mechanism that gives rise to the universal algebraic convergence behavior. In a pushed front, in contrast, the nonlinearity "pushes the leading edge forward" and convergence is exponential.

To illustrate this discussion by a concrete example, we note that when the function $f(\phi)$ in the nonlinear diffusion equation is of the form

$$
f=f_{\epsilon}(\phi)=\epsilon \phi+\phi^{n+1}-\phi^{2 n+1}, \quad n>0 .
$$

we can rely on known analytic solutions for $\Phi_{v}$. In this case, the state $\phi=0$ is (meta)stable for $\epsilon<0$. For $0<\epsilon<(n+1) / n^{2}$, the selected front is pushed, and for $\epsilon>(n+1) / n^{2}$, it is pulled (see Section 2 and Appendix C).

At this point, a brief explanation of our use of the word "metastable" may be appropriate. For systems with a Lyapunov function, the word metastable is often used in physics to denote a linearly stable state, which does not correspond to the absolute minimum of the Lyapunov function or "free energy". A domain wall or front between the absolutely stable and a metastable state then moves into the metastable domain; one may therefore loosely call a linearly stable state "metastable", if it is invaded by another "more stable" state through the motion of a domain wall or front.

The understanding of the two different dynamical mechanisms of pushing and pulling in the nonlinear diffusion equation (1.1) lays the basis for the analysis of equations like those listed in Table 1. The essential step towards a generalization of the leading edge representation (1.9) is done by a saddle point analysis, that identifies which Fourier modes of linear perturbations of the unstable state will dominate the long time dynamics. This analysis yields the parameters $v^{*}, \lambda^{*}$, the diffusion constant $D$ and possible higher order terms required for the leading edge representation.

\footnotetext{
12 Note though, that this subtle point is quite important - as we shall see, the saddle point or pinch point analysis gives precisely the wrong prefactor for the leading $1 / t$ convergence term because this boundary condition is not satisfied.
} 


\subsection{Sketch of method and results on front relaxation in the pulled regime}

Bramson's method [74] to calculate algebraic convergence is specifically adapted to equations of type (1.1). It is based on a representation of the diffusion equation by Brownian processes, which are evaluated probabilistically. Instead, we construct the asymptotic convergence trajectory towards a known asymptotic state by solving the differential equations in a systematic asymptotic expansion which, though non-rigorous, extends immediately to higher order equations. Our approach leads to exact results since the expansion parameter are inverse powers of the time $t$, so these terms become arbitrarily small in the asymptotic regime.

The idea of the method is that in a pulled front, the speed is essentially set in the leading edge, where linearization of the equation of motion about the unstable state is justified. This leading edge has to be connected to what we will refer to as the interior part of the front, defined to be the region where we have to work with the full nonlinear equation. For the interior, we use the fact that for large times the shape of the converging front will resemble the asymptotic front, and thus can be expanded about it. We also explicitly make use of the fact that the initial state $\phi(x, 0)$ for large $x$ is steeper than the asymptotic front profile $\Phi^{*}=\Phi_{v^{*}}$ in the leading edge, i.e., $\phi(x, 0) \mathrm{e}^{\lambda^{*} x} \rightarrow 0$ as $x \rightarrow \infty$. The structure of the problem then dictates the expansion in $1 / \sqrt{t}$.

The structure of the expansion in $1 / \sqrt{t}$ is the only real input of the analysis; its self-consistency becomes clear a posteriori and it can be motivated from the earlier work on the long time expansion of the Green's function of the linearized equations. Equivalently, the self-consistency emerges from the observation that the equation governing the convergence towards the asymptotic front profile (1.9) reduces essentially to a diffusion equation in the leading edge of the front. The derivation of the exact results summarized in Table 2 is essentially based on this ansatz.

The shape convergence is also obtained explicitly from our analysis. The crucial input for the analysis is the right frame and structure to linearize about. At first sight, a natural guess would be that for large times, the actual shape of the front $\phi(x, t)$ should be linearizable about the shape of the asymptotic front $\Phi^{*}\left(x-v^{*} t\right)$. However, the algebraic velocity convergence (1.3) implies that if a converging front profile $\phi$ is close to the asymptotic uniformly translating front profile $\Phi^{*}\left(x-v^{*} t\right)$ at sometime $t_{0}$, the distance between the actual profile and $\Phi^{*}$ will diverge at large times $t$ as $X(t)=-\left(3 / 2 \lambda^{*}\right) \ln \left(t / t_{0}\right)+\cdots$. This result which is illustrated in Fig. 1 implies that if we want to linearize $\phi$ about $\Phi^{*}$ at all times, we have to move $\Phi^{*}$ along with the non-asymptotic velocity $v(t)(1.3)$ of the converging front. A crucial step for the analysis is thus to linearize about $\Phi^{*}\left(\xi_{X}\right)$ in a coordinate system:

$$
\left(\xi_{X}, t\right), \quad \xi_{X} \equiv \xi-X(t)=x-v^{*} t-X(t),
$$

moving with the converging front. If we expand $\phi$ about $\Phi^{*}\left(\xi_{X}\right)$ with $\xi_{X}$ from (1.11) and then resum, we find that

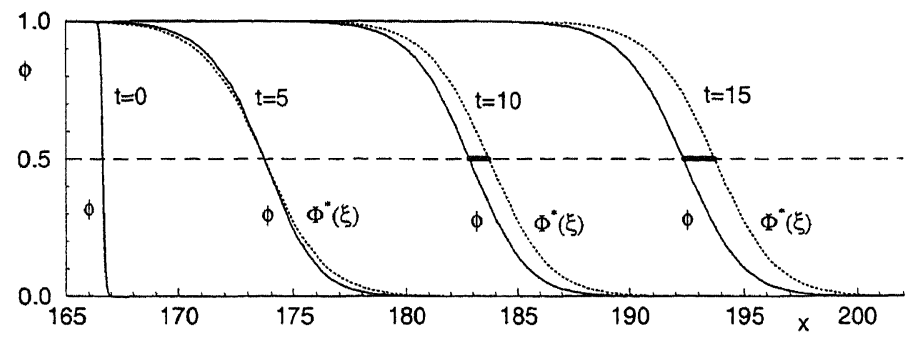

Fig. 1. Illustration of the fact, that even though the shape of a front profile is quite close to $\Phi^{*}$, the position of a front is shifted logarithmically in time relative to the uniformly translating profile $\Phi^{*}(\xi)$. Solid lines: evolution of some initial condition $\phi(x, 0)$ of the form $(4.2)$ under $\partial_{t} \phi=\partial_{x}^{2} \phi+\phi-\phi^{3}$ at times $t=5,10,15$. Dotted lines: evolution of $\phi(x, t)=\Phi^{*}(\xi), \xi=x-2 t$, at times $t=5,10,15$. $\Phi^{*}$ is placed such that the amplitude $\phi=\frac{1}{2}$ coincides with that of $\phi(x, t)$ at time $t=5$. The logarithmic temporal shift is indicated by the fat line. 
the interior shape of the front is given by

$$
\phi(x, t)=\Phi_{v(t)}\left(\xi_{X}+x_{0}\right)+\mathrm{O}\left(\frac{1}{t^{2}}\right)
$$

for $\xi_{X} \ll \sqrt{4 D t}$. $x_{0}$ expresses the translational degree of freedom of the front. The uniformly translating front $\Phi_{v}(\xi)$ is a solution of the ordinary differential equation for the uniformly translating profile $\phi(x, t)=\Phi_{v}(x-v t)$, but with $v$ replaced by the instantaneous value $v(t)$ of the velocity. For example, for the nonlinear diffusion equation $(1.1), \Phi_{v}(\xi)$ is the solution of

$$
-v \partial_{\xi} \Phi_{v}(\xi)=\partial_{\xi}^{2} \Phi_{v}(\xi)+f\left(\Phi_{v}(\xi)\right)
$$

Eq. (1.12) also confirms that to leading order the interior is slaved to the slow dynamics of the leading edge. The transient profiles $\Phi_{v(t)}$ in (1.12) propagate with velocity $v(t)$ smaller than $v^{*}$ according to (1.3).

For the special case of Eq. (1.1) it is well known (see also Section 2) that when constructing a front $\Phi_{v}$ starting from $\Phi_{v}=1$ at $\xi \rightarrow-\infty$, it eventually will become negative for finite $\xi$, whenever $v<v^{*}$, and that globally such fronts either do not exist or are dynamically unstable, depending on the properties of $f$ for negative $\phi$. However, only the positive part of $\Phi_{v(t)}$ from $\xi \rightarrow-\infty$ up to $\xi \ll \sqrt{t}$ plays a role as a transient. That the convergence trajectory is approximately given by $\Phi_{v(t)}$, was already observed numerically in equations of type (1.1) by Powell et al. [67]. Our analytical derivation of this result actually holds for a larger class of equations, but at the same time we find that it only holds up to a correction term of order $1 / t^{2}$. This non-universal correction is always non-vanishing.

For $\xi_{X} \gg \sqrt{4 D t}$, the transient crosses over to

$$
\phi(x, t)=\alpha \xi_{X} \exp \left\{-\lambda^{*} \xi_{X}-\frac{\xi_{X}^{2}}{4 D t}\right\}\left(1+\mathrm{O}\left(\frac{1}{\sqrt{t}}\right)+\mathrm{O}\left(\frac{1}{\xi_{X}}\right)\right) .
$$

The analytical expression for the universal correction of order $1 / \sqrt{t}$ in (1.14) is given by Eqs. (3.65) and (3.67) for the nonlinear diffusion equation and is generalized by Eqs. (5.39) or (5.69), while the correction of order $1 / t$ will depend on initial conditions, and is thus non-universal.

A crucial insight implemented above is that the front consists of different dynamical regions which have to be matched to each other. The situation is sketched in Fig. 2. For a pulled front, the Gaussian region (1.14) of the leading edge essentially determines the velocity while the front interior (1.12) is slaved to leading order. The Gaussian region might be preceded by a region of "steepness" $\lambda$ being conserved in time which for sufficiently steep initial conditions $\lambda>\lambda^{*}$ has no dynamical importance (where the steepness $\lambda$ is defined in Eq. (2.6) below). Likewise, for flat initial conditions, the dynamics is dominated by the conserved $\lambda$ region, while pushed dynamics is dominated by the front

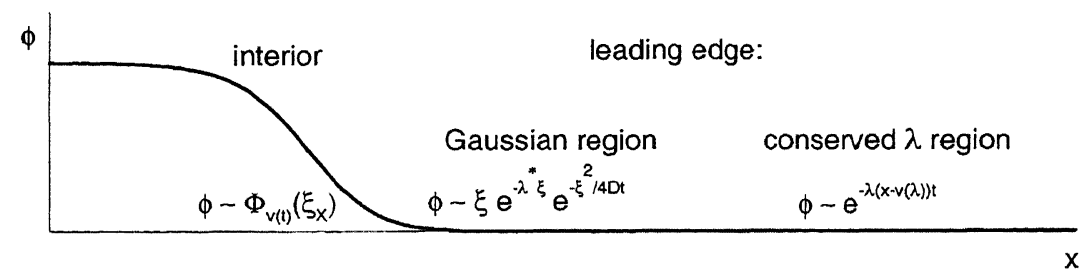

Fig. 2. Sketch of a relaxing pulled front with the different dynamical regions: the interior is the nonlinear region, the leading edge is the region linearized about the unstable state. Depending on the initial conditions, the leading edge might still consist of two different regions: a Gaussian region and a region of conserved steepness $\lambda$. For $\lambda>\lambda^{*}$ (defining "sufficiently steep" initial conditions), the (intermediate) asymptotic Gaussian region determines the velocity relaxation. 
Table 2

The central results on the universal algebraic relaxation towards uniformly translating pulled fronts, see also Fig. 2 . These results apply to steep initial conditions in the nonlinear diffusion equation in the pulled regime (Case IV of Table 4, see Sections 3 and 4) and to more general equations (see Section 5)

Height independent velocity:

$\Rightarrow$ Use the coordinate:

Front for $\xi_{X} \ll \sqrt{4 D t}$ (front interior)

Front for $\xi_{X} \gg \sqrt{4 D t}$ (leading edge)

$$
\begin{aligned}
& \begin{aligned}
v(t) & =v^{*}+\dot{X} \\
& =v^{*}-\frac{3}{2 \lambda^{*} t}\left(1-\sqrt{\frac{\pi}{\left(\lambda^{*}\right)^{2} D t}}\right)+O\left(\frac{1}{t^{2}}\right)
\end{aligned} \\
& \text { where the saddle point analysis of the linearized } \\
& \text { equation yields } v^{*}, \lambda^{*}, D, \text { cf. Table } 5 \text { or Eqs. (1.4)- }
\end{aligned}
$$
(1.7).

$\xi_{X}=x-v^{*} t-X(t)$

$$
\begin{aligned}
& \begin{aligned}
\phi(x, t) & =\Phi_{v(t)}\left(\xi_{X}\right)+O\left(\frac{1}{t^{2}}\right) \\
& =\Phi^{*}\left(\xi_{X}\right)+\dot{X} \eta_{\mathrm{sh}}\left(\xi_{X}\right)+O\left(\frac{1}{t^{2}}\right)
\end{aligned} \\
& \text { where } \Phi_{v}(\xi) \text { solves } \phi(x, t)=\Phi_{v}(x-v t) \\
& \text { and } \eta_{\mathrm{sh}}(\xi)=\delta \Phi_{v}(\xi) /\left.\delta v\right|_{v^{*}}
\end{aligned}
$$

$\phi(x, t)=\alpha \xi_{X} e^{-\lambda^{*} \xi X} e^{-\xi_{X}^{2} /(4 D t)}+\ldots$

interior. In both of these cases, the intermediate Gaussian region is absent. For the nonlinear diffusion equation (1.1), the different cases are discussed in Section 2 and summarized in Table 4. Our results (1.3)-(1.14) are universal in four ways:

- They are independent of which "height" or level curve is being tracked to define the front velocity.

- The predicted convergence behavior is independent of the precise initial conditions, provided they decay quicker than $\mathrm{e}^{-\lambda^{*}|x|}$ far in the unstable regime.

- The leading edge behavior (1.3) and (1.14) is independent of the precise nonlinearities. For Eq. (1.1), the constants $v^{*}, \lambda^{*}$ and $D$ depend on $f^{\prime}(0)$ only. For the more general equations, these constants are completely determined by the saddle point expansion in the equation linearized about the unstable state.

- If we analyze general equations like those listed in Table 1, our prediction for the interior part of the front (1.12) stays unchanged, as long as the front speed stays determined by the linearization about the unstable state, i.e., the front stays pulled, and as long as the state behind the front stays homogeneous. The effect of the nonlinearities just gets absorbed in appropriate functions $\Phi_{v}$.

The results summarized in this section are the most central new results of this paper. They are summarized, for easy reference in Table 2.

\subsection{Organization of the paper}

Before embarking on our explicit calculation of the velocity and shape convergence in the pulled regime, we review in Section 2 rather well known results on the multiplicity, stability and convergence of pushed fronts in the nonlinear diffusion equation, and discuss how far these results can be extended to pulled fronts or fronts emerging from "flat" initial conditions. Since the convergence towards pulled fronts cannot be derived by linear stability 
analysis, we set the stage for Section 3 by introducing the leading edge transformation. In the central Section 3 , the detailed analysis of pulled front relaxation in the nonlinear diffusion equation (1.1) is given. The detailed numerical simulations that fully confirm our analytical predictions are presented in Section 4 . In this section, we also pay attention to the specific problems of spatial discretization and system size arising in the numerical solution of pulled front propagation. In Section 5, we extend our analysis to more general equations, discuss the example equations listed in Table 1, and present numerical results, again in excellent agreement with our analytical predictions. Here the picture of a new center manifold theorem for pulled front propagation emerges. We then close the main body of the paper with a summary and outlook in Section 6.

Since this is a long paper with a large number of detailed results of various types, and since we have made an attempt to make our results accessible for readers from different fields, we introduce Table 3 as a "helpdesk" for the

Table 3

A guide through the paper for the efficient reader who wants to read about specific results only, or who already has some background knowledge on the problem of front propagation into unstable states

\begin{tabular}{|c|c|}
\hline \multicolumn{2}{|l|}{ THE READER'S HELPDESK } \\
\hline If $\ldots . .$. & then our advice is...... \\
\hline $\begin{array}{l}\text {... you want to focus right away on the } \\
\text { relaxation calculation without analyzing } \\
\text { what a stability calculation tells and does } \\
\text { not tell about the relaxation of pulled } \\
\text { fronts, }\end{array}$ & $\begin{array}{l}\text {... if you know what is meant with the } \\
\text { "pulled" velocity } v^{*} \text { you can start with } \\
\text { Section } 3 \text { immediately; if not, read Sec- } \\
\text { tion } 2.5,2.6 \text { and possibly Section } 5.3 .1 \\
\text { first. }\end{array}$ \\
\hline $\begin{array}{l}\text {... you are already familiar with previ- } \\
\text { ous ideas concerning front selection in } \\
\text { the physics literature, but want to get } \\
\text { an idea of our change of emphasis and of } \\
\text { the new detailed results of this paper, }\end{array}$ & $\begin{array}{l}\text {... to skim Section } 2.1 \text { for notation and } \\
\text { a summary of the most important back- } \\
\text { ground material, to then check the ap- } \\
\text { propriate Sections of } 2 \text { on points which } \\
\text { are not clear from Section } 2.1 \text {, and then } \\
\text { to proceed to Section } 3 \text {. }\end{array}$ \\
\hline $\begin{array}{l}\text {... you (mainly) want to read about the } \\
\text { connection between stability, selection } \\
\text { and relaxation, }\end{array}$ & $\begin{array}{l}\ldots \text { to read Section } 2 \text { with Table } 4 \text { and for } \\
\text { the generalization Sections } 5.2 \text { and } 5.3 \\
\text { with their appendices. }\end{array}$ \\
\hline $\begin{array}{l}\text {... you only want to get an idea of the } \\
\text { conceptual basis of the algebraic conver- } \\
\text { gence, }\end{array}$ & $\begin{array}{l}\text {... to read Section } 3.1 \text { and possibly Sec- } \\
\text { tions } 5.3-5.5 \text { for the arguments concern- } \\
\text { ing higher order partial differential equa- } \\
\text { tions or other types of equations. }\end{array}$ \\
\hline $\begin{array}{l}\text {... you are unfamiliar with the concept of } \\
\text { pulled velocity } v^{*} \text { for higher order equa- } \\
\text { tions and want to know how it is deter- } \\
\text { mined, }\end{array}$ & $\begin{array}{l}\text {... to read Sections } 3.1 \text { and } 5.3 .1 \text { (and } \\
\text { possibly parts of Sections } 5.4 \text { and } 5.5 \text { ). }\end{array}$ \\
\hline $\begin{array}{l}\text {... you just want to see the numerical sup- } \\
\text { port for the algebraic relaxation predic- } \\
\text { tion from Tables } 2 \text { and } 5 \text {, or want to read } \\
\text { about the numerical intricacies of study- } \\
\text { ing the pulled front convergence, }\end{array}$ & $\begin{array}{l}\text {... read Section } 4 \text { on the nonlinear diffu- } \\
\text { sion equation and Section } 5.6 \text { for higher } \\
\text { order and coupled equations. }\end{array}$ \\
\hline $\begin{array}{l}\text {... you just want a toolkit for when to } \\
\text { apply the predictions from Tables } 2 \text { and } \\
5 \text {, }\end{array}$ & ... to read Section 6.3 . \\
\hline
\end{tabular}


reader who wants to focus on a particular aspect of the front propagation problem only, or who wants to get only an idea of the essential ingredients of our approach and the main results.

We finally note that a brief sketch of our results can be found in [82] and the lecture notes [83]. Later extensions of the present work can be found in $[37,46,48,72,73,99]$.

\section{Stability, selection and convergence in the nonlinear diffusion equation}

In this section, we provide the necessary background information on fronts propagating into unstable states by reviewing a number of results on the multiplicity and stability of uniformly translating front solutions of the nonlinear diffusion equation $[32,36,38,49-51,54,61,63,65-70,84,85,87,88,90-92,114-116] .{ }^{13}$ We also summarize to what extent the linear stability analysis of these uniformly translating fronts allows us to solve the selection problem, i.e., to determine the basins of attraction of these solutions in the space of initial conditions and for different nonlinearities $f$, and to what extent it allows us to answer the related question of the convergence rate and mechanism. It will turn out that the linear stability analysis fails to explain how pulled fronts emerging from sufficiently steep initial conditions relax to their asymptotic speed and profile. This sets the stage for a different approach to pulled fronts by introducing the leading edge representation.

\subsection{Statement of problem and essential concepts}

In Sections 2-4, we analyze the nonlinear diffusion equation

$$
\partial_{t} \phi(x, t)=\partial_{x}^{2} \phi+f(\phi),
$$

where $f(\phi)$ is assumed to be continuous and differentiable. For studying front propagation into unstable states, it is convenient to take

$$
f(0)=0=f(1), \quad f^{\prime}(0)=1, \quad f(\phi)>0 \text { for all } 0<\phi<1,
$$

so that in the interval $[0,1], f(\phi)$ has one unstable state at $\phi=0$ and only one stable state at $\phi=1$. Eq. (2.2) implies that $f^{\prime}(1)<0$. Note, that we have specified the behavior of $f(\phi)$ only on the interval $0 \leq \phi \leq 1$. This is all we need since it can be shown by comparison arguments [51 ${ }^{14}$ that an initial state with $0 \leq \phi(x, 0) \leq 1$ for all $x$ conserves this property in time under the dynamics of (2.1) and (2.2).

In passing, we note that for a nonlinearity like (1.10), a general equation of the form

$$
\partial_{\tau} \varphi=D \partial_{y}^{2} \varphi+F_{\epsilon}(\varphi), \quad F_{\epsilon}(0)=0=F_{\epsilon}\left(\varphi_{s}\right), \quad F_{\epsilon}^{\prime}(0)=\epsilon, \quad \varphi_{s}>0,
$$

results. It allows $\epsilon$ to take either sign. For $\epsilon<0$, the state $\phi=0$ is linearly stable, for $\epsilon>0$, it is unstable. Fronts propagating into metastable states $(\epsilon<0)$ will sometimes also be discussed briefly for comparison. If $\epsilon>0,(2.3)$ transforms to the normal form $(2.1)$ as

$$
t=\epsilon \tau, \quad x=\left(\frac{\epsilon}{D}\right)^{1 / 2} y, \quad \phi=\frac{\varphi}{\varphi_{s}}, \quad f(\phi)=\frac{F_{\epsilon}(\varphi)}{\epsilon \varphi_{s}} .
$$

Hence velocities transform as $\mathrm{d} x / \mathrm{d} t=[\mathrm{d} y / \mathrm{d} \tau] /(D \epsilon)^{1 / 2}$.

\footnotetext{
${ }^{13}$ See, e.g., Section 3.2 in [86].

${ }^{14} \mathrm{~A}$ brief overview of comparison type arguments can be found in the Appendix of Ref. [61].
} 
The front propagation problem can now be stated as follows. Consider some initial condition $0 \leq \phi(x, 0) \leq 1$ with

$$
\lim _{x \rightarrow \infty} \phi(x, 0)=0, \quad \phi(x, 0)>0 \text { for some } x,
$$

that evolves under the equation of motion (2.1) with (2.2) into a front propagating to the right. Which time-independent profile and which velocity will this front approach asymptotically as time $t \rightarrow \infty$, if any? How quick will the convergence to this asymptotic front be? Can we identify the mechanisms that generate such dynamical behavior? Can we rephrase it in such terms that we can generalize results to equations other than (2.1)? These questions essentially concern the nature of the front selection mechanism.

As is well known, the answers to these questions depend on more specific properties of the initial condition as well as of the nonlinearity $f(\phi)$. For the nonlinear diffusion equation, the answer to the selection problem is known in full rigor, but we will only review here those concepts which are important in a more general context and which play a role in the subsequent relaxation analysis. We now briefly outline these main concepts and results and explain them in more detail in the rest of Section 2.

Existence of a family of front solutions. For front propagation into unstable states, the selection problem is different and more intricate than for bistable fronts (fronts between two linearly stable states), since when one solves the ODE for the uniformly translating profile $\phi(x-v t)=\Phi_{v}(\xi)$ one finds that there is a family of fronts solutions parametrized by the continuous variable $v$ that are possible attractors of the dynamics. This is in contrast to the situation for bistable fronts where the selected velocity $v$ is obtained simply as a nonlinear eigenvalue problem.

Steepness of a front. Most of our discussion focuses more than earlier work on the central and unifying role of the steepness $\lambda$ of the leading edge of a front, defined as the asymptotic exponential decay rate:

$$
\phi(x, t) \stackrel{x \rightarrow \infty}{\sim} \mathrm{e}^{-\lambda x} \Leftrightarrow \lambda=-\lim _{x \rightarrow \infty}\left(\frac{\partial \ln \phi}{\partial x}\right) .
$$

When $\phi(x, t)$ decays faster than exponentially as $x \rightarrow \infty$, this implies $\lambda=\infty$.

Pulled and pushed fronts. The family of uniformly translating and dynamically stable fronts $\Phi_{v}$ can be uniquely parametrized either by the velocity $v$ or by the spatial decay rate or steepness $\lambda$. The difference between pushed and pulled solutions is especially clear if we characterize them by $\lambda$. A given nonlinearity $f$ defines two particular steepnesses: $\lambda_{\text {sel }}$ which characterizes the pushed and pulled front solutions and $\lambda_{\text {steep }}$ which characterizes the basin of attraction of these so-called selected fronts. The front solution with $\lambda=\lambda_{\text {sel }}>1$ defines the pushed front, while the pulled one has $\lambda=\lambda_{\text {sel }}=\lambda^{*}=1$. The continuous family of dynamically stable front solutions that exists in addition to these selected fronts is parametrized by $\lambda<\lambda_{\text {steep }} \leq 1$. The nature and construction of the fronts is discussed in more detail in Section 2.2, together with a simple property of pulled fronts which will play an important role in our later relaxation analysis, namely the fact that the asymptotic large time profile of a pulled front is as $\Phi_{v^{*}}(\xi) \sim \xi \mathrm{e}^{-\lambda^{*} \xi}$ for $\xi \gg 1$.

We will characterize also an initial condition by its steepness $\lambda$ and call it a sufficiently steep initial condition, if $\phi(x, t=0)$ decays to zero exponentially faster than $\mathrm{e}^{-\lambda_{\text {steep }} x}$ for some $\lambda_{\text {steep }} \leq 1$, i.e.,

$$
\text { sufficiently steep : } \quad \phi(x, 0) \stackrel{x \rightarrow \infty}{<} \mathrm{e}^{-\lambda x} \text { for some } \lambda>\lambda_{\text {steep }},
$$

otherwise we call it flat:

$$
\text { flat : } \quad \phi(x, 0) \stackrel{x \rightarrow \infty}{\sim} \mathrm{e}^{-\lambda x}, \quad \lambda<\lambda_{\text {steep }}
$$

How $\lambda_{\text {steep }}$ is determined by $f(\phi)$, will be discussed in Section 2.4 . We will see that always $0<\lambda_{\text {steep }} \leq 1$ for 
Eq. (2.1), and in particular that for pulled fronts

pulled fronts : $\quad \lambda_{\text {steep }}=\lambda^{*}=1$,

while for pushed fronts $\lambda_{\text {steep }}<1$. The criterion (2.7) for steepness includes all initial conditions with bounded support or, e.g., the initial condition $\phi(x, 0)=\theta(-x)$ with $\theta$ the step function.

Note that the intermediate case $\phi(x, 0) \sim x^{-\nu} \mathrm{e}^{-\lambda^{*} x}$ is neither sufficiently steep nor flat, according to our definitions. In Section 3, we shall recover Bramson's [74] observation that such special initial conditions also lead to a $1 / t$ relaxation of the velocity profile, but with a $\nu$-dependent prefactor for $v<2$.

Conservation of steepness. In Section 2.5, we discuss what we term conservation of steepness: if an initial condition is characterized by a steepness $\lambda$, then at any finite time the steepness of $\phi(x, t)$ is the same as that of the initial condition $\phi(x, t=0)$. (Note that the limits $t \rightarrow \infty$ and $x \rightarrow \infty$ do not commute.)

The linear stability analysis of front solutions can be performed in detail for the nonlinear diffusion equation. As summarized in Section 2.3, pushed fronts have a gapped spectrum, while pulled fronts have a gapless spectrum within their natural Hilbert space. In the selection analysis, we in general also need perturbations from outside this Hilbert space.

Stability and selection. In Section 2.4, we discuss the connection between the stability of front solutions and the selection mechanism; this connection, which underlies much of the marginal stability scenario $[61,63,65]$, hinges on the fact that the conservation of steepness allows one to relate the steepness of the initial condition to the steepness of the late stage evolution of the front that can be decomposed into an asymptotic front profile plus a linear perturbation. The spectral decomposition of this perturbation is largely determined by the steepness of the initial and the asymptotic state.

Basins of attraction and rate of convergence are also discussed in Section 2.4. Flat initial conditions (2.8) approach a front characterized by their initial $\lambda$. Sufficiently steep initial conditions $(2.7)$ in the pushed regime $\left(\lambda_{\text {sel }}>1\right)$ evolve at late times into a pushed front corrected by linear perturbations that can be represented by eigenfunctions of the stability operator, whose spectrum has a gap. Hence the convergence of a pushed front is exponential in time. In contrast, the rate of convergence of pulled fronts $\left(\lambda_{\text {sel }}=1\right)$ cannot simply be obtained from the spectrum, as it is gapless, and generic perturbations are not spanned by the "natural" eigenfunctions of the spectrum.

Leading edge and interior dominated dynamics. Both the stability analysis and our relaxation analysis bring out the importance of distinguishing leading edge dominated from interior dominated dynamics. The most obvious form of leading edge dominated dynamics results from flat exponential initial conditions (2.8) with finite steepness $\lambda$. In this case, the asymptotic front speed is just the speed

$$
v(\lambda)=\lambda+\frac{1}{\lambda}
$$

with which the exponential tail $\mathrm{e}^{-\lambda x}$ propagates according to the linear dynamical equation

$$
\partial_{t} \phi=\partial_{x}^{2} \phi+\phi+o\left(\phi^{2}\right)
$$

This equation is obtained by linearizing about the unstable state $\phi=0$, and is appropriate in the leading edge region. The more important leading edge dominated dynamics occurs, however, for sufficiently steep initial conditions (2.7) converging to a pulled front. As already mentioned, for pulled fronts the asymptotic front speed is just the linear spreading velocity $v^{*}$ determined in the leading edge where the dynamics is essentially governed by the linearized evolution equation. This type of leading edge dominated pulled dynamics occurs when the nonlinearities in $f(\phi)$ are mostly saturating so that they slow down the growth. In passing, we note that 
we rederive in Appendix A the well-known sufficient criterion for pulling in the nonlinear diffusion equation, viz.

$$
f^{\prime}(0)=\max _{0 \leq \phi \leq 1} \frac{f(\phi)}{\phi},
$$

with the help of a transformation that we call the leading edge transformation $[73,82]$. This form of a proof is generalizable to some other equations $[72,73,99]$. Pulled fronts are actually at the margin of leading edge domination: although the linearized equation (2.11) is sufficient to determine $v_{\text {sel }}=v^{*}=2$, we will see in Section 3 that the convergence towards this velocity is governed by a non-trivial interplay of the dynamics in the leading edge and the "slaved" interior.

Leading edge dominated dynamics contrasts with interior dominated dynamics, which occurs when the nonlinear function $f(\phi)$ is such that steep initial conditions give rise to pushed fronts. For interior dominated or pushed dynamics, $v_{\text {sel }}$ is associated with the existence of a strongly heteroclinic orbit in the phase space associated with $\Phi_{v}(\xi)$ (Section 2.2). This means that the whole nonlinearity $f(\phi)$ is needed for constructing $v_{\text {sel }}$, not only the linearization $f^{\prime}(0)$ about the unstable state. The linear stability analysis of Section 2.3 implies that pushed fronts converge exponentially in time to their asymptotic speed (Section 2.4). This type of dynamics extends smoothly towards fronts propagating into metastable states, i.e., towards $\epsilon<0$ in (2.3).

While in this section, we consider the nonlinear diffusion equations (2.1) and (2.2) only, the straightforward extension to generalized PDEs of the form $F\left(\phi, \partial_{x} \phi, \partial_{x}^{2} \phi, \partial_{t} \phi\right)=0$ can be found in Appendix B.

In the following subsections, the above assertions are further substantiated. Readers familiar with most of the concepts and results listed above can proceed to Section 3 .

\subsection{Uniformly translating fronts: candidates for attractors and transients}

In this section, we recall some well known properties $[49-51,61,63,65,69,93,94,117]^{15}$ of uniformly translating front solutions of the nonlinear diffusion equations (2.1) and (2.2). We transform to a coordinate system moving with uniform velocity $v:(x, t) \rightarrow(\xi, t), \xi=x-v t$, so that the temporal derivative transforms as $\left.\partial_{t}\right|_{x}=\left.\partial_{t}\right|_{\xi}-\left.v \partial_{\xi}\right|_{t}$. For a front $\phi(x-v t)=\Phi_{v}(\xi)$ translating uniformly with velocity $v$, the time derivative vanishes in the comoving frame $\left.\partial_{t}\right|_{\xi} \Phi_{v}=0$, and so $\Phi_{v}(\xi)$ obeys the ordinary differential equation

$$
\partial_{\xi}^{2} \Phi_{v}+v \partial_{\xi} \Phi_{v}+f\left(\Phi_{v}\right)=0 .
$$

In view of the initial condition (2.5), throughout this paper we will focus on the right-moving front and hence we impose the boundary conditions

$$
\Phi_{v}(\xi) \rightarrow 1 \text { for } \xi \rightarrow-\infty, \quad \Phi_{v}(\xi) \rightarrow 0 \text { for } \xi \rightarrow \infty .
$$

Close to the stable state $\phi=1$, the differential equation can be linearized about $\phi=1$ and solved explicitly. The general local solution is a linear combination of $\exp \left\{-\tilde{\lambda}_{ \pm} \xi\right\}$ with

$$
\tilde{\lambda}_{ \pm}=\frac{1}{2}\left(v \pm\left(v^{2}-4 f^{\prime}(1)\right)^{1 / 2}\right) .
$$

According to (2.2), $f^{\prime}(1)$ is negative. Thus for any real $v, \tilde{\lambda}_{+}$is positive and $\tilde{\lambda}_{-}$is negative. With the convention (2.14), only the negative root is acceptable. So

$$
\Phi_{v}(\xi)=1 \pm \exp \left\{-\tilde{\lambda}_{-}\left(\xi-\xi_{0}\right)\right\}+o\left(\exp \left\{-2 \tilde{\lambda}_{-} \xi\right\}\right) \text { for } \xi \rightarrow-\infty .
$$

\footnotetext{
${ }^{15}$ We stress that we claim no originality here. In the physics literature, this type of analysis has appeared in various places, quite often in relation to Ginzburg-Landau or mean-field type approaches (see, e.g., Refs. $[79,95,97]$ ).
} 
The free integration constant multiplying $\exp \left\{-\tilde{\lambda}_{-} \xi\right\}$ here has been decomposed into a sign \pm and a free parameter $\xi_{0}$ accounting for translation invariance. Apart from translation invariance, there are two solution for $\Phi_{v}$ close to $\phi=1$ distinguished by \pm .

A global view of the nature and multiplicity of solutions can be obtained with a well-known simple particle-in-apotential analogy. This analogy has of course been exploited quite often in various types of approaches [63,95-97] and only works for the nonlinear diffusion equation, not for equations with higher spatial derivatives; for these, we have to rely on a construction of solutions as trajectories in phase space as sketched around Eq. (2.24).

The particle-in-a-potential analogy is based on the identification of Eq. (2.13) with the equation of motion of a classical particle with friction in a potential. One identifies $\Phi_{v}$ with a spatial coordinate, $\xi$ with time, $v$ with a friction coefficient, and $f$ with the negative force, $f=-$ force $=\partial_{\phi} V(\phi)$ derived from the potential $V(\phi)=\int^{\phi} \mathrm{d} \phi^{\prime} f\left(\phi^{\prime}\right)$. The potential has a maximum at $\phi=1$ and a minimum at $\phi=0$. The construction of $\Phi_{v}$ is equivalent to the motion of a classical particle with "friction" $v$ in this potential, where at "time" $-\infty$ the particle is at rest at the maximum of $V$. Obviously, for any positive "friction" $v>0$, the particle will never reach the minimum at $\phi=0$, if it takes off from the maximum at $\phi=1$ towards $\phi>1$. It will always reach $\phi=0$ if it takes off towards $\phi<1$. Thus for every $v>0$, there is a unique uniformly translating front (unique up to a translation), that starts as (2.14) and reaches $\phi=0$ monotonically. Close to $\phi=1$ it is given by the - branch in Eq. (2.16).

Let us be more specific on how $\phi=0$ is approached. If the "friction" $v$ is sufficiently large, the motion of the particle will be overdamped when it first approaches $\phi=0$, it will reach $\phi=0$ only for "time" $\xi \rightarrow \infty$, and form a monotonic front over the whole $\xi$ axis. This behavior continues down to a critical value of the "friction" $v_{\mathrm{c}}$. It defines the critical velocity $v_{\mathrm{c}}$ as the smallest velocity at which $\Phi_{v}(\xi)$ monotonically reaches $\Phi_{v}(\xi) \rightarrow 0$ at $\xi \rightarrow \infty$. (As we will discuss in Section 2.3, a uniformly translating front $\Phi_{v}$ is dynamically stable if and only if $v \geq v_{\mathrm{c}}$.) If $v<v_{\mathrm{c}}$, the particle will reach $\phi=0$ at a finite "time" $\xi$ and cross it. What then happens, depends on $f(\phi)$ for negative arguments. If $f^{\prime}(0)=1$ for both positive and negative arguments $\phi$ as in the case of the nonlinearities (1.2) or (1.10), the particle might oscillate a finite or an infinite number of times through $\phi=0$ and reach $\phi=0$ asymptotically for $\xi \rightarrow \infty$ as

$$
\Phi_{v}(\xi)= \begin{cases}A_{v} \mathrm{e}^{-\lambda_{-} \xi}+B_{v} \mathrm{e}^{-\lambda_{+} \xi} & \text { for } v>2, \\ (\alpha \xi+\beta) \mathrm{e}^{-\lambda^{*} \xi} & \text { for } v=v^{*}=2, \\ C_{v} \mathrm{e}^{-\lambda_{0} \xi} \cos k\left(\xi-\xi_{2}\right) & \text { for }|v|<2,\end{cases}
$$

where

$$
\begin{aligned}
& \lambda_{ \pm}(v)=\lambda_{0}(v) \pm \mu(v) \quad(v>2), \quad \lambda_{0}(v)=\frac{1}{2} v \quad(\text { all } v), \\
& \mu(v)=\frac{1}{2}\left(v^{2}-4\right)^{1 / 2} \quad(v>2), \quad k(v)=\frac{1}{2}\left(4-v^{2}\right)^{1 / 2} \quad(v<2), \\
& \lambda^{*}=\lambda_{0}\left(v^{*}\right)=\lambda_{ \pm}\left(v^{*}\right)=1 \quad\left(v=v^{*}=2\right) .
\end{aligned}
$$

The solution (2.17) of the equation linearized about $\phi=0$ contains two free parameters for every $v$. These parameters are determined by the unique approach of the front $\Phi_{v}$ from $\phi=1$ and will, in general, both be non-vanishing. The special value $v^{*}=2$ is determined by linearization about the unstable state. As can be seen from (2.17), it is a lower bound on the critical velocity $v_{c}$. At this value of the velocity, the two roots $\lambda_{+}$and $\lambda_{-}$coincide. As a result, the asymptotic profile is not the sum of two exponentials, but an exponential times a first order polynomial in $\xi$.

Depending on the nonlinearity $f$, the critical $v_{c}$ can be determined by two different mechanisms that turn out to distinguish pushed $\left(v_{\mathrm{c}}>v^{*}\right)$ or pulled $\left(v_{\mathrm{c}}=v^{*}\right)$ fronts. Suppose first that upon lowering $v$ the front solutions $\Phi_{v}$ remain monotonic till $v=v^{*}$. In this case, $v_{\mathrm{c}}=v^{*}$ is determined by the equation linearized about the unstable 
state, and we will see, that sufficiently steep initial conditions (2.7) evolve into pulled fronts. A second possibility is the following. At very large $v$, the front solution is certainly monotonic, since in the particle-on-the-hill analogy the particle slowly creeps to the minimum of the potential for large "friction" $v$. Hence $A_{v}$ in (2.17) is positive for large $v$. Now, depending on the nonlinearities, it may happen upon lowering $v$ that at some velocity $v=v^{\dagger}, A_{v^{\dagger}}=0$. The front is non-monotonic for $v<v^{\dagger}$ as $A_{v}$ will be negative for $v<v^{\dagger}$. Hence in this case $v_{\mathrm{c}}=v^{\dagger}$ and pushed fronts result. The pushed velocity $v^{\dagger}$ thus emerges from the global analysis of the whole nonlinear front, and not only from linearization about the unstable state.

For uniformly translating pulled fronts, we will use the short-hand notation $\Phi^{*} \equiv \Phi_{v^{*}}$. For large $\xi$ they are asymptotically

$$
\Phi^{*}(\xi) \equiv \Phi_{v^{*}}(\xi) \stackrel{\xi \rightarrow \infty}{\sim} \xi \mathrm{e}^{-\xi}
$$

since in general the coefficient $\alpha$ in (2.17) is non-zero. This particular form will in Section 3 turn out to have important consequences for the convergence of pulled fronts: it determines the prefactor of the $1 / t$ relaxation term.

For fronts with velocity $v>v^{*}$, the smaller $\lambda$ will dominate the large $\xi$ asymptotics, so generically

$$
\Phi_{v}(\xi) \stackrel{\xi \rightarrow \infty}{\sim} \mathrm{e}^{-\lambda-\xi}
$$

However, for a front solution with velocity $v^{\dagger}$, we have $A_{v} \dagger=0$, and so

$$
\Phi^{\dagger}(\xi) \equiv \Phi_{v} \dagger(\xi)^{\xi \rightarrow \infty} \mathrm{e}^{-\lambda+\xi}
$$

An alternative formulation that can be generalized to higher order equations is the following. A construction of front solutions of Eq. (2.13) is equivalent to a construction of trajectories in a phase space $\left(\Phi_{v}, \Psi_{v} \equiv \partial_{\xi} \Phi_{v}\right)$ in which the flow is given by

$$
\partial_{\xi}\left(\begin{array}{c}
\Phi_{v} \\
\Psi_{v}
\end{array}\right)=\left(\begin{array}{c}
\Psi_{v} \\
-v \Psi_{v}-f\left(\Phi_{v}\right)
\end{array}\right) .
$$

Front solutions correspond to trajectories between the fixed points $\left(\Phi_{v}, \Psi_{v}\right)=(1,0)$ and $(0,0)$. These are thus heteroclinic orbits in phase space. Out of the $(1,0)$ fixed point come two trajectories in opposite directions along one eigenvector according to (2.16). When we follow the direction for which $\Phi_{v}$ decreases for increasing $\xi$, its behavior near the $(0,0)$ fixed point is given by (2.17). Now, since the flow depends continuously on $v$, so will $A_{v}$ and $B_{v}$ in (2.17). For large $v, A_{v}$ is positive, and from the construction of the flow in phase space one sees that $A_{v}$ may change sign on lowering $v$. The largest $v$ with $A_{v}=0$ determines the change from monotonic to non-monotonic fronts. At $v=v^{\dagger}$, the trajectory flows into the stable $(0,0)$ fixed point along the most strongly contracting eigendirection this is precisely what is expressed in (2.23). For this reason, the solution $\Phi^{\dagger}$ is referred to by Powell et al. [67] as a strongly heteroclinic orbit. In [66], this solution was referred to as "the nonlinear front solution".

In summary, the main results of the preceding analysis are:

1. For every $v \geq v_{\mathrm{c}}$, there is a uniformly translating front $\Phi_{v}$ with velocity $v$, which monotonically connects $\phi=1$ at $\xi \rightarrow-\infty$ to $\phi=0$ at $\xi \rightarrow \infty$. All $\Phi_{v}$ with these properties are uniquely determined by $v$ up to translation invariance.

2. For every $0<v<v_{\mathrm{c}}$, there is a unique front solution $\Phi_{v}$ that translates uniformly with velocity $v$, and that monotonically connects $\phi=1$ at $\xi \rightarrow-\infty$ to $\phi=0$ at some finite $\xi=\bar{\xi}$.

3. Depending on the nonlinearities, the change from monotonic to non-monotonic behavior can either occur at the velocity $v^{*}$, with $v^{*}=2$ for (2.1) and (2.2), or at a larger velocity $v^{\dagger}: v_{\mathrm{c}}=\max \left[v^{*}, v^{\dagger}\right]$. If $v^{\dagger}$ exists, it is the largest velocity at which there is a strongly heteroclinic orbit. 


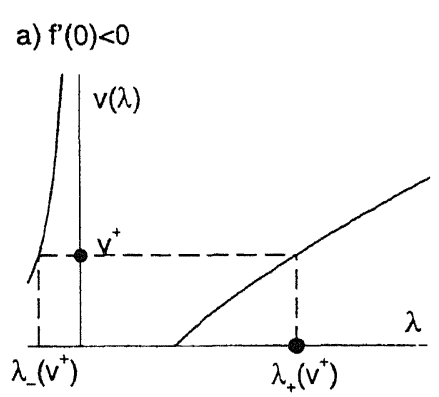

b) $f^{\prime}(0)>0, v_{c}=v^{+}>v^{*}$

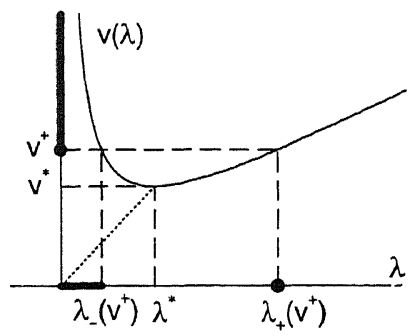

c) $f^{\prime}(0)>0, v_{c}=v^{*}$

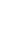

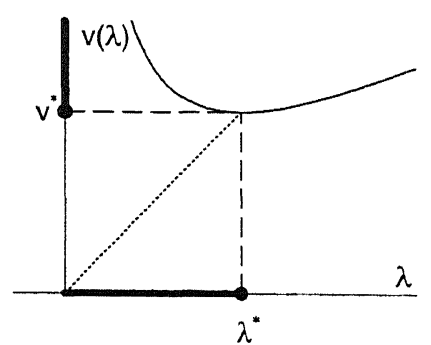

Fig. 3. Steepness $\lambda(2.6)$ versus velocity $v(\lambda)=\lambda+f^{\prime}(0) / \lambda$ with solid line for real $\lambda$ and dotted line for real part of complex $\lambda$. $v^{\dagger}$ is the pushed velocity derived from global analysis, $v^{*}$ the linear spreading velocity. A fat line or point on the axes denotes the possible attractors $\Phi_{v}(x-v t)$ of the dynamics, parametrized either by velocity $v$ or by steepness $\lambda$ : (a) the case $f^{\prime}(0)<0$ corresponding to front propagation into a (meta)stable state. In this case, there is a unique attractor with velocity $v_{\text {sel }}=v^{\dagger}$ and steepness $\lambda_{\text {sel }}=\lambda_{+}\left(v^{\dagger}\right)$; (b) and (c) the case $f^{\prime}(0)>0$ corresponding to front propagation into an unstable state. In this case there is a continuum of attractors parametrized by $v \geq v_{\mathrm{c}}$; (b) the pushed regime: $v_{\text {sel }}=v_{\mathrm{c}}=v^{\dagger}>v^{*}$. The steepness $\lambda_{\text {sel }}=\lambda_{+}\left(v^{\dagger}\right)$ of the steepest attractor is isolated just as in case (a). There is a continuous family of fronts parametrized by $0<\lambda<\lambda_{\text {steep }}=\lambda_{-}\left(v^{\dagger}\right)$; (c) the pulled regime: $v_{\text {sel }}=v_{\mathrm{c}}=v^{*}$. The steepness $\lambda^{*}=\lambda_{\text {steep }}=\lambda_{\text {sel }}$ of the steepest attractor is at the margin of the $\lambda$-continuum of attractors.

The results for invasion into either metastable $\left(f^{\prime}(0)<0\right)$ or unstable states $\left(f^{\prime}(0)>0\right)$ and for $v_{\mathcal{c}}=v^{\dagger}>v^{*}$ and $v_{c}=v^{*}$ are summarized in $v(\lambda)$ plots in Fig. 3, which show the multiplicity of stable uniformly translating fronts $\Phi_{v}$ parametrized by either $v$ or $\lambda$.

The results of this section play a role in the subsequent analysis:

- There are important connections [61] between the properties of the uniformly translating front solutions and the stability of these fronts (see Section 2.3). In particular, front solutions with velocity $v \geq v_{c}$ are dynamically stable and possible attractors of the long-time dynamics. Fronts with velocity $v<v_{c}$ either do not exist or are unstable.

- The results for front selection can be easily formulated in terms of the properties of these uniformly translating solutions $[61,63,65]$ : for sufficiently steep initial conditions, the dynamically selected velocity coincides with $v_{\mathrm{c}}: v_{\mathrm{sel}}=v_{\mathrm{c}}$. If $v_{\text {sel }}=v^{\dagger}$, we speak of the pushed regime, while if $v_{\text {sel }}=v^{*}$ we speak of pulled fronts.

- We will see in Section 3 that the positive monotonic part of the front solutions $\Phi_{v}(\xi)$ with velocity $v<v^{*}$ plays a role in the convergence behavior in the interior region of pulled fronts. Note, however, that while a solution $\Phi_{v}(\xi)$ of the ODE has according to (2.17) an oscillatory leading edge for large $\xi$, that causes the dynamic instability of these solutions, the relaxing front is approximated by $\Phi_{v(t)}$ only in the interior front region and crosses over to a different functional form in the leading edge. This behavior is in agreement with the conservation of positivity of the solution in a nonlinear diffusion equation, if the initial condition was positive.

All arguments essentially also apply to higher order equations, though then the positivity and monotonicity properties of the solutions loose their distinguished role.

\subsection{Linear stability analysis of moving front solutions}

To study the linear stability of a uniformly translating front $\Phi_{v}$, we linearize about it in the frame $\xi=x-v t$ moving with the constant velocity $v$, by writing

$$
\phi(\xi, t)=\Phi_{v}(\xi)+\eta(\xi, t) .
$$

Inserting (2.25) into (2.1), we find to linear order the equation of motion for $\eta(\xi, t)$

$$
\partial_{t} \eta=\mathcal{L}_{v} \eta+\mathrm{O}\left(\eta^{2}\right)
$$


with the linear operator

$$
\mathcal{L}_{v}=\partial_{\xi}^{2}+v \partial_{\xi}+f^{\prime}\left(\Phi_{v}(\xi)\right)
$$

$\mathcal{L}_{v}$ is not self-adjoint, so left-and right eigenfunctions will differ. The trouble is caused by the linear derivative $v \partial_{\xi}$. It can be removed by the following transformation $[61,85]$ :

$$
\begin{aligned}
& \psi=\mathrm{e}^{v \xi / 2} \eta, \\
& \mathcal{H}_{v}=-\mathrm{e}^{v \xi / 2} \mathcal{L}_{v} \mathrm{e}^{-v \xi / 2} .
\end{aligned}
$$

$\mathcal{H}_{v}$ is the linear Schrödinger operator

$$
\mathcal{H}_{v}=-\partial_{\xi}^{2}+V(\xi), \quad V(\xi)=\frac{1}{4} v^{2}-f^{\prime}\left(\Phi_{v}(\xi)\right)
$$

and the equation of motion (2.26) transforms to

$$
-\partial_{t} \psi=\mathcal{H}_{v} \psi+\mathrm{O}\left(\psi^{2} \mathrm{e}^{-v \xi / 2}\right) .
$$

This Schrödinger problem is, of course, well known to physicists [86] (see also [85,87-91]), as long as $\psi$ lies in the natural Hilbert space of $\mathcal{H}_{v}$.

However, the transformation (2.28) and (2.29) increases the weight of the leading edge $(\xi \rightarrow \infty)$ by a factor $\mathrm{e}^{v \xi / 2}$, while it enhances convergence at $\xi \rightarrow-\infty$. Therefore, only perturbations with

$$
\lim _{\xi \rightarrow \infty}|\eta| \mathrm{e}^{\lambda_{0}(v) \xi}<\infty \quad \text { with } \quad \lambda_{0}(v)=\frac{1}{2} v
$$

are spanned by the eigenfunctions within the conventional Hilbert space of $\mathcal{H}_{v}$. For the selection analysis in Section 2.4 below, this function space in general is not sufficient. As it is discussed in detail in Appendix D, one can construct eigenmodes of $\mathcal{L}_{v}$ outside the Hilbert space defined by (2.32). With this extension of the function space, scalar products of arbitrary eigenfunctions might be divergent, so one looses the efficient tool of projection onto eigenfunctions by taking inner products. Nevertheless, in most cases generic perturbations still can be decomposed into these eigenfunctions, except in the case of pulled fronts: the linear perturbation $\eta$ of a sufficiently steep front $\phi \sim \mathrm{e}^{-\lambda \xi}$ with $\lambda>1(2.7)$ about the asymptotic pulled front $\Phi^{*} \sim(\alpha \xi+\beta) \mathrm{e}^{-\xi}(2.17)$ and (2.21) will decay asymptotically as

$$
\eta=\phi-\Phi^{* \xi}{ }^{\xi 1}-(\alpha \xi+\beta) \mathrm{e}^{-\xi}
$$

Since there is only one zero mode of translation with a slightly different asymptotic behavior

$$
\mathcal{L}_{v} \eta_{0}=0, \quad \eta_{0}=\partial_{\xi} \Phi^{* \xi} \stackrel{1}{\sim}-(\alpha \xi+\beta-\alpha) \mathrm{e}^{-\xi},
$$

the asymptotics of $\eta$ cannot fully be decomposed into eigenfunctions. Here the double root structure of the leading edge with $\alpha \neq 0$ plays a crucial role, as it later will do again.

The most important conclusions from the present discussions and the detailed Appendix D are:

1. Non-monotonic fronts are intrinsically unstable, and generically will not be approached by any initial condition.

2. Monotonic fronts propagating with velocity $v$ are stable against perturbations steeper than $\mathrm{e}^{-\lambda_{-}(v) \xi}$.

3. Perturbations $\eta$ about pushed fronts $\Phi^{\dagger}$ that decay more rapidly than $\lambda_{-}\left(v^{\dagger}\right)$ have a gapped spectrum (see Eq. (D.14)). The same holds for perturbations about fronts $\Phi_{v}$ with a velocity $v>v^{\dagger}$, if their steepness is larger than $\lambda_{-}(v)$. 
4. The spectrum of pulled fronts is gapless and cannot be decomposed into eigenfunctions of $\mathcal{H}_{v}$ even outside the conventional Hilbert space.

Before closing this section, we note that although the particle-on-a-hill analogy for $\Phi_{v}$ or the mapping onto the Schrödinger equation for $\eta_{\sigma}$ are insightful and very efficient ways to arrive at our results for existence and stability of uniformly translating front solutions, the analysis by no means relies on these. In fact, much of the phase space analysis can easily be generalized to higher order equations as those shown in Table 1. For example, in the stability analysis of non-monotonic fronts, the discrete set of solutions with $A_{v}=0$ plays a particular role. For equations like the EFK equation from Table 1, monotonicity ceases to be a criterium, but conditions like $A_{v}=0$ defining the so-called strongly heteroclinic solutions continue to play a central role in the stability analysis, as discussed in Appendix $\mathrm{H}$ to Section 5 .

\subsection{Consequences of the stability analysis for selection and rate of convergence; marginal stability}

Suppose now, that we start with an initial condition $\phi(x, 0)$ in the nonlinear diffusion equation (2.1) with a given nonlinearity $f(\phi)$, and then study the ensuing dynamics. What will the linear stability analysis tell us about the asymptotic $(t \rightarrow \infty)$ state and the rate of convergence? It turns out that the issue of selection is more closely related to that of stability than one might expect at first sight. The reason is the conservation of steepness discussed in more detail in Section 2.5 below: If initially at $t=0$ the steepness $\lambda$ defined in (2.6) is non-zero (finite or infinite), then at any finite time $t<\infty$ the steepness is conserved:

$$
\phi(x, 0)^{x \rightarrow \infty} \mathrm{e}^{-\lambda x} \Rightarrow \phi(x, t)^{x \rightarrow \infty} \mathrm{e}^{-\lambda x} \text { for all } t<\infty .
$$

Note that the limits $x \rightarrow \infty$ and $t \rightarrow \infty$ do not commute. We characterize the initial condition by its steepness $\lambda_{\text {init }}$ defined by

$$
\phi(x, t=0) \stackrel{x \rightarrow \infty}{\sim} \mathrm{e}^{-\lambda_{\text {init }} x} .
$$

As a consequence of (2.35), we can use $\lambda_{\text {init }}$ to characterize not only the initial conditions but also the profile at any later time $0 \leq t<\infty$, when the front velocity might be already close to its asymptotic value.

The conservation of steepness (2.35) entails that a front characterized by an initial steepness $\lambda_{\text {init }}$, will be characterized by the same steepness after any finite time, so also at a late stage when the velocity and shape of a front are close to their asymptotic limits. At such a late stage, the front $\phi$ can be decomposed into a possible attractor $\Phi_{v}(x-v t)$ of the dynamics plus a linear perturbation $\eta$ as in (2.25). We characterize the attractor $\Phi_{v}$, that we

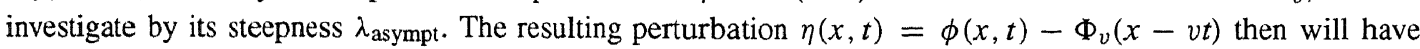
steepness

$$
\lambda_{\eta}=\min \left[\lambda_{\text {init }}, \lambda_{\text {asympt }}\right] .
$$

Whether the perturbation $\eta$ will grow or decay, that means, whether $\Phi_{v}$ with a particular velocity $v$ is the attractor of the evolution of $\phi$ or not, is determined by the decomposition of the perturbation $\eta$ into eigenmodes of the linear operator. Whether this spectrum has growing eigenmodes, depends on the operator and the function space defined by the steepness $\lambda_{\eta}$. With the tools of the stability analysis from Appendix D, the selection question can therefore be rephrased purely in terms of $\lambda_{\eta}, \lambda_{\text {asympt }}$ and the two steepnesses $\lambda_{\text {steep }}$ and $\lambda_{\text {sel }}$ characterizing the nonlinearity $f:$ for pushed fronts

$$
\lambda_{\text {steep }}=\lambda_{-}\left(v^{\dagger}\right), \quad \lambda_{\text {sel }}=\lambda_{+}\left(v^{\dagger}\right), \quad v_{\text {sel }}=v^{\dagger},
$$


Table 4

Table of initial conditions and nonlinearities, resulting in relaxation cases I-IV from Appendix E. Fronts at all times $t$ are characterized by their steepness $\lambda(2.6)$ in the leading edge, and an arrow $\rightarrow$ indicates the evaluation of the quantity for $t \rightarrow \infty$. The nonlinearity only enters through the existence of a strongly heteroclinic orbit $\Phi_{v}(x-v t)$ with $v_{\text {sel }}=v^{\dagger}>v^{*}$ (see Section 2.2) or its non-existence (then $v_{\text {sel }}=v^{*}$ ). $v_{\text {sel }}$ determines $\lambda_{ \pm, 0}\left(v_{\mathrm{sel}}\right)$ as in (2.18), which in turn classifies the initial conditions. Pushed or pulled dynamics are special cases of interior or leading edge dominated dynamics for steep initial conditions. Cases I-III are treated in Appendix E with stability analysis methods and generically show exponential relaxation. Case IV is not amenable to stability analysis methods. It shows algebraic relaxation and is treated from Section 3 on

\begin{tabular}{|c|c|c|c|}
\hline \multirow{3}{*}{$\begin{array}{c}\text { INITIAL } \\
\text { CONDITION } \\
\phi(x, 0) \stackrel{x \rightarrow \infty}{\sim} e^{-\lambda_{\text {init }} x} \\
(\text { definition: } \\
\left.\lambda_{0, \pm}=\lambda_{0, \pm}\left(v_{\text {sel }}\right)\right)\end{array}$} & \multicolumn{3}{|c|}{$\begin{array}{c}\text { NONLINEARITY } \\
f(\phi)\end{array}$} \\
\hline & $\begin{array}{l}\text { metastable } \\
\left(f^{\prime}(0)<0\right) \text { : }\end{array}$ & \multicolumn{2}{|c|}{$\begin{array}{c}\text { unstable } \\
\left(f^{\prime}(0)>0\right)\end{array}$} \\
\hline & $\begin{array}{c}v_{s e l}=v^{\dagger}>0 \\
v^{*}=0\end{array}$ & $\begin{array}{c}\text { pushed regime: } \\
v_{\text {sel }}=v^{\dagger}>v^{*} \\
v^{*}=2 \sqrt{f^{\prime}(0)}\end{array}$ & $\begin{array}{c}\text { pulled regime: } \\
v_{\text {sel }}=v^{*} \\
v^{*}=2 \sqrt{f^{\prime}(0)}\end{array}$ \\
\hline $\begin{array}{c}\text { steep: } \\
\lambda_{0}<\lambda_{\text {init }}\end{array}$ & \multicolumn{2}{|c|}{$\begin{array}{c}\text { Case I: } \\
\text { pushed dynamics, } \\
\lambda \longrightarrow \lambda_{+}\left(v^{\dagger}\right) \\
v(t)=v^{\dagger}+O\left(e^{-\sigma t}\right)\end{array}$} & $\begin{array}{c}\text { Case IV: } \\
\text { pulled dynamics, } \\
\lambda \longrightarrow \lambda^{*} \\
v(t)=v^{*}+O(1 / t)\end{array}$ \\
\hline $\begin{array}{c}\text { steep: } \\
\lambda_{-}<\lambda_{\text {init }}<\lambda_{0}\end{array}$ & \multicolumn{2}{|c|}{$\begin{array}{c}\text { Case II: } \\
\text { pushed dynamics, } \\
\lambda \longrightarrow \lambda_{+}\left(v^{\dagger}\right) \\
\text { generically: } v(t)=v^{\dagger}+O\left(e^{-\sigma t}\right)\end{array}$} & $\begin{array}{c}\text { not applicable } \\
\left(\lambda_{ \pm}\left(v^{*}\right)=\lambda_{0}=\lambda^{*}\right)\end{array}$ \\
\hline $\begin{array}{c}\text { flat: } \\
0<\lambda_{\text {init }}<\lambda_{-}\end{array}$ & $\begin{array}{c}\text { not applicable } \\
\left(\lambda_{-}\left(v^{\dagger}\right)<0\right)\end{array}$ & \multicolumn{2}{|c|}{$\begin{array}{c}\text { Case III: } \\
\text { leading edge dominated dynamics, } \\
\lambda \longrightarrow \lambda_{\text {init }}<\lambda_{-}\left(v^{\dagger}\right) \\
v \longrightarrow v\left(\lambda_{\text {init }}\right)>v_{\text {sel }} \\
\text { generically: } v(t)=v\left(\lambda_{\text {init }}\right)+O\left(e^{-\sigma t}\right)\end{array}$} \\
\hline
\end{tabular}

and for pulled fronts

$$
\lambda_{\text {steep }}=\lambda_{\text {sel }}=\lambda^{*}, \quad v_{\text {sel }}=v^{*},
$$

in the notation of (2.18)-(2.20).

A detailed discussion of the question to what extent one can understand the selection and rate of convergence of fronts following this line of analysis is given in Appendix $\mathrm{E}$ and summarized in Table 4. The two most important conclusions for our purposes concern fronts evolving from sufficiently steep initial conditions:

1. The gapped spectrum of the conventional Hilbert space for pushed fronts implies that the relaxation towards pushed front solutions is exponential in time.

2. Even after extending linear stability analysis beyond the Hilbert space, it is not possible to derive the rate of convergence of pulled fronts from the stability spectrum, since it is gapless, and generic perturbations cannot be decomposed into eigenmodes of the linear stability operator even in an enlarged functions space.

We finally note that the usual marginal stability viewpoint is to characterize the family of stable front solutions $\Phi_{v}$ by the velocity $v$; from this perspective, the front velocity $v_{\text {sel }}$ selected by the sufficiently steep initial conditions is at the edge of a continuous spectrum of stable solutions with $v \geq v_{\text {sel }}$. In this sense, both the pushed and the pulled attractors are marginally stable $[61,63,65]$. The picture changes, however, when the attractors are not characterized 
by the velocity $v$, but by their asymptotic steepness $\lambda$ (see Fig. 3). The pulled front then still is at the margin of a continuous spectrum, while the pushed front is isolated just like the bistable front.

\subsection{The dynamics of the leading edge of a front}

In this section, we reconsider the dynamics in the leading edge in more detail, first to demonstrate the conservation of steepness expressed by (2.35), second to clarify the dynamics that ensues from flat initial conditions, and third to lay the basis for the quantitative analysis of the relaxation of pulled fronts in Section 3.

\subsubsection{Equation linearized about $\phi=0$}

When we analyze the leading edge region of the front, where $|\phi| \ll 1$, to lowest order, we can neglect $o\left(\phi^{2}\right)$ in (2.11) and analyze

$$
\partial_{t} \phi=\partial_{x}^{2} \phi+\phi
$$

We first explore the predictions of this equation, before exploring the corrections due to the nonlinearity $f$ in Section 2.5.2.

Eq. (2.40) is a linear equation, so the superposition of solutions again is a solution. A generic solution is, e.g., an exponential $\mathrm{e}^{-\lambda x}$. It will conserve shape and propagate with velocity $v(\lambda)=\lambda+1 / \lambda(2.10)$ :

$$
\phi(x, t) \sim \exp \{-\lambda[x-v(\lambda) t]\} .
$$

The minimum of $v(\lambda)$ is given by $v^{*}=v\left(\lambda^{*}=1\right)=2$.

Consider now a superposition of two exponentials $c_{1} \mathrm{e}^{-\lambda_{1} x}+c_{2} \mathrm{e}^{-\lambda_{2} x}$. Without loss of generality, we can assume the maximum velocity to be $v_{\max }=\max \left[v\left(\lambda_{1}\right), v\left(\lambda_{2}\right)\right]=v\left(\lambda_{1}\right)$. In the coordinate system $\xi_{1}=x-v\left(\lambda_{1}\right) t$, the temporal evolution then becomes

$$
\begin{aligned}
& \phi(x, t)=c_{1} \mathrm{e}^{-\lambda_{1} \xi_{1}}+c_{2} \mathrm{e}^{-\lambda_{2} \xi_{1}} \mathrm{e}^{-\sigma t}, \\
& \sigma=\lambda_{2}\left(v\left(\lambda_{1}\right)-v\left(\lambda_{2}\right)\right)>0 .
\end{aligned}
$$

Clearly, the contribution of $\lambda_{2}$ decays on the timescale $1 / \sigma$, and so for large times $\gg 1 / \sigma$, the velocity of a so-called level curve of $\phi=$ const. $>0$ in an $x, t$ diagram will approach $v\left(\lambda_{1}\right)$ and the profile will converge to $\mathrm{e}^{-\lambda_{1} \xi_{1}}$ (see [75] for a similar type of analysis). The steepness of the leading edge at $\xi \rightarrow \infty$, on the other hand, will be given by $\lambda_{\min }=\min \left[\lambda_{1}, \lambda_{2}\right]$ for all times $t<\infty$.

This simple example already backs up much of our discussion of perturbations outside the Hilbert space in Appendix E that apply to the Cases II and III in Table 4:

1. The limits $\xi \rightarrow \infty$ and $t \rightarrow \infty$, in general, do not commute.

2. The steepness $\lambda=\min _{i}\left[\lambda_{i}\right]$ is a conserved quantity at $x \rightarrow \infty$ and $t<\infty$. As the explicit example of [102] shows, for equations for which one can derive a comparison theorem, the conservation of steepness can easily be derived rigorously.

3. The velocity of a constant amplitude $\phi=$ const. $>0$ will be governed by the quickest mode present $v=$ $\max _{i}\left[v\left(\lambda_{i}\right)\right]$ at large times $t \gg 1$.

Let us now analyze initial conditions steeper than any exponential. Quite generally, an initial condition $\phi(x, 0)$ evolves under (2.40)) as ${ }^{16}$

$$
\phi(x, t)=\int_{-\infty}^{\infty} \mathrm{d} y \phi(y, 0) \frac{\exp \left\{-\left[(x-y)^{2}-4 t^{2}\right] /(4 t)\right\}}{(4 \pi t)^{1 / 2}} .
$$

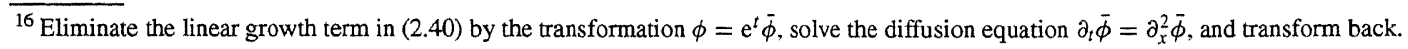


Assume for simplicity, that the initial condition $\phi(y, 0)$ is strongly peaked about $y=0$, so that for large times, we can neglect the spatial extent of the region where $\phi(y, 0) \neq 0$ initially. Upon introducing the coordinate $\xi=x-2 t$ we get

$$
\phi(x, t) \propto \frac{\exp \left\{-\xi-\xi^{2} /(4 t)\right\}}{\sqrt{t}} \text { for } t \gg 1 .
$$

This general expression leads to three important observations:

1. The steepness of the leading edge characterized by $\lambda=\infty$ at $\xi \rightarrow \infty$ indeed is conserved for all finite times $t<\infty$.

2. At finite amplitudes $\phi=$ const. $>0$ and large times $t$, the steepness of the front propagating towards $\xi \rightarrow \infty$ approaches $\lambda^{*}=1$ and the velocity approaches $v^{*}=2$.

3. Eq. (2.45) furthermore implies that a steep initial condition like $\phi(y, 0)$ approaches the asymptotic velocity $v^{*}$ as

$$
v(t)_{\text {lin }}=v^{*}+\dot{\xi}_{h}=2-\frac{1}{2 t}+\mathrm{O}\left(\frac{1}{t^{2}}\right),
$$

where we defined the position $\xi_{h}(t)$ of the amplitude $h$ in the comoving frame $\xi=x-2 t$ as $\phi\left(\xi_{h}(t), t\right)=h$. Eq. (2.46) is then obtained simply by solving $\ln \phi=-\xi_{h}-\xi_{h}^{2} / 4 t-\frac{1}{2} \ln t=$ const.

This algebraic convergence is consistent with the gapless spectrum of linear perturbations, and as such it identifies the missing link in the analysis of the relaxation of pulled fronts. However, Bramson's work [74] shows that the qualitative prediction of convergence as $1 / t$ is right, but the coefficient of $1 / t$ is wrong. In fact, the mathematical literature [53] has established (2.46) as an upper bound for the velocity of a pulled front in a nonlinear diffusion equation. The algebraic convergence clearly comes from the $1 / \sqrt{t}$ prefactor characteristic of the fundamental Gaussian solution of the diffusion Eq. (2.45) - this qualitative mechanism will be found to be right in Section 3.

We finish our discussion of solutions of the linearized equation (2.40) with another illustrative example. After the discussion of the solution (2.41) one might be worried about initial conditions with $\lambda \gg 1$. Such an initial condition is steep according to our definition, so it should approach the velocity $v^{*}$. But according to (2.41), it approaches the larger velocity $v(\lambda)$. However, even in the framework of the linearized equation, this paradox can be resolved: an initial condition $\mathrm{e}^{-\lambda x}$ on the whole real axis is, of course, unphysical, and we in fact only want this behavior at $x \gg 1$, where $\phi$ is small. Let us therefore truncate the exponential for small $x$ by writing, e.g., $\phi(x, 0)=\theta(x) \mathrm{e}^{-\lambda x}$, with $\theta$ the step function. Insertion into (2.44) yields the evolution

$$
\phi(x, t)=\exp \{-\lambda[x-v(\lambda) t]\} \frac{1+\operatorname{erf}[(x-2 \lambda t) / \sqrt{4 t}]}{2},
$$

where erf $x=2 \pi^{-1 / 2} \int_{0}^{x} \mathrm{~d} t \mathrm{e}^{-t^{2}}$ is the errorfunction. For $t \gg 1$, the crossover region where $x \approx 2 \lambda t$ separates two different asymptotic types of behavior:

$$
\phi(x, t) \approx \begin{cases}\exp \{-\lambda[x-v(\lambda) t]\} & \text { for } x \gg 2 \lambda t, \\ \frac{\exp \left\{-(x-2 t)-(x-2 t)^{2} / 4 t\right\}}{\sqrt{4 \pi t} \lambda(1-x /(2 \lambda t))} & \text { for } x \ll 2 \lambda t .\end{cases}
$$

In the region of $x \gg 2 \lambda t$, we find our previous solution (2.41) with conserved leading edge steepness and velocity $v(\lambda)$, while in the region of $x \ll 2 \lambda t$ we essentially recover (2.45), with $\xi=x-2 t$.

Considering the three different velocities $-v(\lambda)$ for the region of conserved $\lambda, v^{*}=2$ for the "Gaussian" region behind, and $2 \lambda$ for the crossover region between the two asymptotes - the distinction between flat and steep initial conditions now comes about quite naturally: 
1. For flat initial conditions, we have $\lambda<1$, and an ordering of velocities as $2 \lambda<v^{*}<v(\lambda)$. The crossover region then moves slower than both asymptotic regions, so for large times the region of finite $\phi$ will be dominated by $\exp \{-\lambda[x-v(\lambda) t]\}$.

2. For steep initial conditions, we have $\lambda>1$, and the velocities order as $v^{*}<v(\lambda)<2 \lambda$. The crossover region then will move quicker than both asymptotic regions, and the region of finite $\phi$ will be dominated by $\exp \left\{-\xi-\xi^{2} /(4 t)\right\} / \sqrt{t}$, where $\xi=x-2 t$.

We finally note that the above results can also be reinterpreted in terms of the intuitive picture advocated in [63,65]: the group velocity $v_{\mathrm{gr}}(\lambda)=\mathrm{d} v(\lambda) / \mathrm{d} \lambda$ of a near exponential profile in the leading edge is, according to (2.10), negative for $\lambda<1$ and positive for $\lambda>1$. In this way of thinking, the region with steepness $\lambda$ in the case considered above expands when $\lambda<1$ since the crossover region moves back in the comoving frame (case 1), and it moves out of sight towards $\xi \rightarrow \infty$ for $\lambda>1$ (case 2 ), since the crossover region moves faster than the local comoving frame.

\subsubsection{Leading edge representation of the full equation}

Just as the linear stability analysis of the front was insufficient to cover the full dynamical behavior of the nonlinear diffusion equation (2.1) and in particular the dynamics of the leading edge, so is the linearized equation (2.40). In Section 3, we will see that only through joining these complementary approaches, we can gain a quantitative understanding of the convergence of steep initial conditions towards a pulled front $\Phi^{*}$.

The shortcomings of the linearized equation (2.40) become quite clear by confronting it with what we will call the leading edge representation of the full equation (2.1):

$$
\partial_{t} \psi=\partial_{\xi}^{2} \psi+\vec{f}(\psi, \xi)
$$

where we transformed with

$$
\psi=\phi \mathrm{e}^{\lambda^{*} \xi}, \quad \xi=x-v^{*} t .
$$

The parameters are $\lambda^{*}=\frac{1}{2} v^{*}=f^{\prime}(0)^{1 / 2}$. This transformation eliminates the terms of order $\psi$ and $\partial_{\xi} \psi$ from the linear part of the equation. The nonlinearity is

$$
\bar{f}(\psi, \xi)=\mathrm{e}^{\lambda^{*} \xi}\left(f\left(\psi \mathrm{e}^{-\lambda^{*} \xi}\right)-f(0)-f^{\prime}(0) \psi \mathrm{e}^{-\lambda^{*} \xi}\right)=o\left(\psi^{2} \mathrm{e}^{-\lambda^{*} \xi}\right) .
$$

This transformation is quite comparable to the transformation of a linear perturbation $\eta$ into the Schrödinger picture as in (2.27) and (2.28). Here, however, we transform the full nonlinear equation, and not only the linearization about some asymptotic solution.

For, e.g., $f(\phi)=\phi-\phi^{3}$ we have $\bar{f}=-\psi^{3} \mathrm{e}^{-2 \xi}$. When we neglect $\bar{f}$ in (2.49), the equation is equivalent to the linearization about $\phi=0(2.40)$. The linearization is correct for $\xi \gg 1$, but the presence of the crossover towards a different behavior for smaller $\xi$ has important consequences for the solutions of the full nonlinear diffusion equation.

In particular, for the leading edge of a pulled front $\Phi^{*} \sim(\alpha \xi+\beta) \mathrm{e}^{-\xi}(2.17)$, we generically find $\alpha \neq 0$ and accordingly the leading edge behavior (2.21). This leading edge behavior will play a central role in Section 3 . In Section 2.2, we derived $\alpha \neq 0$ from the uniqueness of the trajectory in phase space, i.e., from the construction of the whole front from $\phi=0$ up to $\phi=1$. We now will give a different argument for $\alpha \neq 0$ from the analysis of (2.49), that does not rely on constructing the whole solution up to $\phi=1$.

The front $\Phi^{*}$ propagates uniformly with velocity $v^{*}=2$, so in the frame $\xi=x-2 t$, it is stationary. $\Psi^{*}=\Phi^{*} \mathrm{e}^{\xi}$ then solves

$$
\partial_{\xi}^{2} \Psi^{*}+\bar{f}\left(\Psi^{*}, \xi\right)=0 .
$$


The boundary conditions (2.14) for $\Phi^{*}$ imply for $\Psi^{*}$ :

$$
\Psi^{*}(\xi) \sim \begin{cases}\alpha \xi+\beta & \text { for } \xi \rightarrow \infty, \\ \mathrm{O}\left(\mathrm{e}^{\lambda^{*} \xi}\right) & \text { for } \xi \rightarrow-\infty .\end{cases}
$$

The solution $\Psi^{*}=\alpha \xi+\beta$ for $\xi \rightarrow \infty$ can directly be derived from (2.52) and the condition that $\Phi^{*}$ vanishes at $\xi \rightarrow \infty$. Now integrate (2.52) over the real $\xi$ axis, and find

$$
\alpha=-\int_{-\infty}^{\infty} \mathrm{d} \xi \bar{f}\left(\Psi^{*}, \xi\right) .
$$

The integral on the RHS is well defined, since $\bar{f}$ vanishes exponentially, both for $\xi \rightarrow-\infty$ and for $\xi \rightarrow \infty$. Clearly, a nonlinearity $\bar{f} \neq 0$ generically implies $\alpha \neq 0$ and hence the leading edge behavior (2.21) for a pulled $\Phi^{*}$ front. Only for particular nonlinearities $f$, we occasionally find $\alpha=0$ (see (3.67) and Appendix C). Having $\alpha=0$ is obviously only possible if $\bar{f}$ has terms of opposite sign, so that its spatial average vanishes. For the nonlinearity of form $f=\phi-\phi^{k}$ with $k>1(1.2)$, we find $\alpha \neq 0$ always, and in this case the term $\bar{f}$ acts like a localized sink term in the diffusion equation (2.52) for $\psi$. This interpretation is especially useful for the discussion of the non-uniformly translating fronts $[72,73,99]$.

\subsection{Concluding remarks - interior and edge dominated dynamics}

Table 4 summarizes the results of this section for various nonlinearities and initial conditions. For pushed fronts, the stability analysis gives essentially all the ingredients to determine the rate of convergence for pushed fronts. For pulled fronts emerging from sufficiently steep initial conditions one finds, however, (see Case IV of Appendix E) that the linear stability analysis is not the appropriate tool: the spectrum is gapless and the rate of convergence cannot be determined from the spectrum.

The crucial insight for the further analysis is that a relaxing front can be decomposed into different dynamical regions. Linear stability analysis is the appropriate tool for the interior dominated dynamics of a pushed front. Pulled fronts and fronts evolving from "flat" initial conditions are leading edge dominated. This calls for different methods of analysis. The relaxation of pulled fronts emerging from sufficiently steep initial conditions will be addressed in the next section.

\section{Universal pulled convergence of steep initial conditions in the nonlinear diffusion equation}

In the present section, we will combine our understanding of the dynamics of the leading edge and of the interior of a front into one consistent analytical frame, that allows us to calculate the long time convergence of steep initial conditions towards a pulled front - as we discussed in the previous section, the relaxation in this case cannot be obtained from the linear stability analysis of the asymptotic solution. The different dynamical regions of such a front are sketched in Fig. 2. We match an expansion in the interior, that resembles features of the linear stability analysis, to an expansion of the leading edge. Both expansions are asymptotic expansions in $1 / \sqrt{t}$. This approach allows us to derive the power law convergence of the front velocity and the front profile towards $\Phi^{*}$. This convergence is universal in leading and subleading order and we calculate all universal convergence terms analytically. For clarity, we present the detailed calculation for the nonlinear diffusion equation in this section first, and then discuss the generalization in Section 5 . 


\subsection{Observations which motivate our approach}

\subsubsection{Asymptotic steepness of leading edge determines rate of convergence}

Our calculation of the spreading of the leading edge under the linearized equation in Section 2.5 gave qualitatively the right results, but failed to reproduce the quantitative results for the nonlinear equation: inserting sufficiently steep initial conditions (2.7) into the linearized equation (2.11), we found that the asymptotic shape (2.45) approaches $\mathrm{e}^{-\xi}$ times a Gaussian for $t \rightarrow \infty$ and $\xi \gg 1$ and that this implies for the asymptotic convergence that $v(t)_{\operatorname{lin}}=$ $2-1 /(2 t)+\cdots(2.46)$. For the nonlinear equation, we know that the asymptotic front profile $\Phi^{*}$ behaves as $\Phi^{*} \sim \xi \mathrm{e}^{-\xi}$ for $\xi \gg 1(2.21)$ and Bramson [74] has derived with probabilistic methods that $v(t)=2-3 /(2 t)+\cdots$ independent of the height at which the velocity is measured.

How the exact result of Bramson [74] comes out naturally and generally is brought out quite clearly by rephrasing an argument of [65] as follows (see also [70,82]).

Let us work in the leading edge representation (2.49) and (2.50), and let us from here on use the comoving variable $\xi$ specifically for the frame moving with the pulled velocity $v^{*}=2$,

$$
\xi=x-v^{*} t=x-2 t
$$

The fundamental similarity solution of the diffusion-type equation (2.49) for the leading edge variable $\psi=\phi \mathrm{e}^{\lambda^{*} \xi}$ in the region where the nonlinearity can be neglected, is of course the Gaussian

$$
\psi_{0}(\xi, t)=\frac{\exp \left\{-\xi^{2} /(4 t)\right\}}{(4 \pi t)^{1 / 2}}
$$

It reproduces our solution (2.45) for $\phi$. But also any derivative of the Gaussian $\psi_{n}=\partial_{\xi}^{n} \psi_{0}$ solves (2.49) for $\xi \gg 1$. The $\psi_{n} / \psi_{0}$ are simply Hermite polynomials [98]. ${ }^{17}$ In particular, the dipole solution

$$
\psi_{1}(\xi, t)=\partial_{\xi} \psi_{0} \propto \frac{\xi \exp \left\{-\xi^{2} /(4 t)\right\}}{t^{3 / 2}}
$$

also solves the diffusion equation (2.49) for $\xi \gg 1$ and has the proper asymptotics $\Phi \mathrm{e}^{\xi} \propto \xi$ for $t \rightarrow \infty$. Transforming (3.3) back to $\phi$, we find

$$
\phi(x, t) \propto(x-2 t) \exp \left\{-\left[x-2 t+\frac{3}{2} \ln t\right]\right\} \exp \left\{\frac{-(x-2 t)^{2}}{4 t}\right\} .
$$

If we now trace the position $2 t+X_{h}(t)$ of the point where $\phi$ reaches the amplitude $h$ in the original $x$ frame, we find by solving $\phi\left(2 t+X_{h}, t\right)=h$ from (3.4) for $X_{h}(t) \ll(4 t)^{1 / 2}$

$$
v(t)=2+\dot{X}_{h}=2-\frac{3}{2 t}+\cdots,
$$

in agreement with Bramson's result. This indicates that for large times $t \gg 1$ and far in the leading edge $\xi \gg 1$, the converging front is approximately given by (3.3) if $\alpha \neq 0$ in (2.17) - remember that $\alpha \neq 0$ implies that $\Phi^{*}(\xi) \sim \xi \mathrm{e}^{-\xi}$ for large $\xi$. We will see indeed that (3.3) does emerge as the dominant term in a systematic asymptotic expansion in the leading edge region. For reasons explained below it is, however, more convenient to formulate this expansion in a slightly different frame.

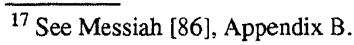




\subsubsection{Interior follows leading edge: uniform convergence}

The above argument shows that the leading $-3 /(2 t)$ velocity correction is due to the diffusion-type dynamics in the leading edge. Why would the convergence to the asymptotic profile be uniform, i.e., be independent of the height $\phi=h$ whose position is tracked? The answer to this question is intuitively quite simple. As $\dot{X}_{h} \simeq-3 /(2 t)$, $X_{h} \simeq-\frac{3}{2} \ln t$. If we compare the position $X_{h_{1}}$ of a height $h_{1}$ in the leading edge $\left(h_{1} \ll 1\right)$ with a position $X_{h_{2}}$ of a height $h_{2}$ in the interior $\left(h_{2}=\mathrm{O}(1)\right)$ where the dynamics of $\phi$ is described by the nonlinear equation, we will have $X_{h_{2}}=X_{h_{1}}-W\left(h_{1}, h_{2}\right)$, where $W$ is the width of the front between these two heights. Clearly, if $W$ approaches a finite value for long times, we need to have also $X_{h_{2}} \simeq \frac{3}{2} \ln t$ in dominant order as $t \rightarrow \infty$, and hence also $\dot{X}_{h_{2}}=-3 /(2 t)+\cdots$. But an equation of motion like (1.1) has front solutions whose width is finite, so we expect indeed that $W=\mathrm{O}(1)$ for large times. Our analysis will confirm this expectation. In other words, the leading order velocity correction as $-3 /(2 t)$ is set by the dynamics of the leading edge, and because of the finite asymptotic width of the front, the convergence is uniform, i.e., independent of $h$.

\subsubsection{Choose proper frame and subtraction for the interior}

The above observations have another important consequence. After the front has evolved for some time, we will find it self-consistent to assume, that its shape will resemble the asymptotic shape $\Phi^{*}$. If we want to understand the interior part of the front, it might at first sight seem appropriate to linearize the converging front $\phi$ about the asymptotic front $\Phi^{*}$. However, the profile $\Phi^{*}$ propagates uniformly with velocity 2 , while as we saw above, the transient profile $\phi$ propagates with velocity $v^{*}-3 /(2 t)$. Thus, if the interior regions of the $\phi$ - and the $\Phi^{*}$-fronts are at about the same part of space at time $t_{0}$, their distance will diverge as $\frac{3}{2} \ln \left(t / t_{0}\right)$ as $t$ grows! This was already illustrated in Fig. 1. Hence, linearization of $\phi$ about the asymptotic profile $\Phi^{*}$ during the whole time evolution requires to move $\Phi^{*}$ along with the velocity $2-3 /(2 t)+\cdots$ of $\phi$ and not with its proper velocity 2 . Our expansion is therefore based on writing $\phi$ as

$$
\phi(\xi, t)=\Phi^{*}\left(\xi_{X}\right)+\eta\left(\xi_{X}, t\right)
$$

where

$$
\xi_{X}=\xi-X(t)=x-2 t-X(t)
$$

This ansatz anticipates that we need to shift the profile $\Phi^{*}$ an appropriate distance $X(t) \propto \ln t$, and that with a proper choice of $X(t), \eta$ becomes a small and decaying perturbation.

\subsubsection{Choose proper expansions and match leading edge to interior}

We will need two different expansions for the leading edge and for the interior. The expansions have to be chosen such that they can be matched in overlapping intervals through resummation of the expansions.

Since we use the coordinate system (3.7) in the interior, we also should use it in the leading edge. The leading $1 / t$ contribution from the leading edge suggests to expand $\eta$ in the interior as $\eta_{1}\left(\xi_{X}\right) / t+\cdots$, and we shall see indeed that such a form emerges automatically from the ansatz (3.6). The appropriate variable for $\xi_{X} \gg \sqrt{t}$ in the leading edge, on the other hand, is the similarity variable of the diffusion equation

$$
z=\frac{\xi_{X}^{2}}{4 t},
$$

as suggested by (3.2)-(3.4). Expressing $\xi_{X}$ by $z$ and $t$ introduces a dependence on $1 / \sqrt{t}$. We find, that it is actually consistent to expand the interior in powers of $1 / \sqrt{t}$ (instead of $1 / t$ ) times functions of $\xi_{X}$, and the leading edge also in powers of $1 / \sqrt{t}$ times functions of $z$. 
The structure of these expansions is essentially our only input. Given this structure, the leading and subleading order universal terms of the expansions are uniquely determined.

\subsection{Expansion in the interior region}

We first analyze the interior part of the front where $\phi$ varies from close to 0 to close to 1 . We work in the comoving frame $\xi_{X}=x-v^{*} t-X(t)$ of (3.7), where $X$ will have to be determined. We expand $\phi$ about $\Phi^{*}\left(\xi_{X}\right)$ as in (3.6). Because of translation invariance, we have the freedom to fix the position of $\Phi^{*}$ and the zero of the coordinate system by imposing

$$
\phi(0, t)=\frac{1}{2} \quad \text { and } \quad \Phi^{*}(0)=\frac{1}{2} \Rightarrow \eta(0, t)=0 .
$$

For $\Phi^{*}$, one has $\Phi^{*}(-\infty)=1$, and we also assume that $\phi$ approaches 1 for $\xi_{X} \rightarrow-\infty$. This results in the second condition on $\eta$

$$
\lim _{\xi_{X} \rightarrow-\infty} \eta\left(\xi_{X}, t\right)=0
$$

We insert $\phi$ into the equation of motion (1.1), transform $x$ to the coordinate $\xi_{X}$ (3.7) and find for $\eta$ the equation ${ }^{18}$

$$
\partial_{t} \eta=\partial_{\xi}^{2} \eta+v^{*} \partial_{\xi} \eta+\dot{X} \partial_{\xi} \Phi^{*}+f\left(\Phi^{*}+\eta\right)-f\left(\Phi^{*}\right) .
$$

Once $\eta$ is small enough because time has evolved sufficiently long, $f\left(\Phi^{*}+\eta\right)$ can be expanded in $\eta$ and we find

$$
\partial_{t} \eta=\mathcal{L}^{*} \eta+\dot{X} \partial_{\xi} \Phi^{*}+\dot{X} \partial_{\xi} \eta+\frac{1}{2} f^{\prime \prime}\left(\Phi^{*}\right) \eta^{2}+O\left(\eta^{3}\right),
$$

where

$$
\mathcal{L}^{*}=\partial_{\xi}^{2}+v^{*} \partial_{\xi}+f^{\prime}\left(\Phi^{*}\left(\xi_{X}\right)\right)
$$

is the linearization operator (2.27) for $v=v^{*}$.

In Sections 2.5.1 and 3.1.1, we have argued that one expects $\dot{X}(t)=\mathrm{O}\left(t^{-1}\right)$. Asymptotic balancing in (3.12) then requires that the leading order term of $\eta$ is of the same order $\eta=O\left(t^{-1}\right)$. We therefore expand as $\eta=\eta_{1}\left(\xi_{X}\right) / t+\cdots$. We have argued that connecting the interior expansion to the leading edge expansion requires an ordering in powers of $1 / \sqrt{t}$. So we choose the ansatz

$$
\begin{aligned}
& \dot{X}=\frac{c_{1}}{t}+\frac{c_{3 / 2}}{t^{3 / 2}}+\frac{c_{2}}{t^{2}}+\cdots, \\
& \eta\left(\xi_{X}, t\right)=\frac{\eta_{1}\left(\xi_{X}\right)}{t}+\frac{\eta_{3 / 2}\left(\xi_{X}\right)}{t^{3 / 2}}+\cdots .
\end{aligned}
$$

Substitution of the above expansions into (3.13) and ordering in powers of $1 / \sqrt{t}$ yields a hierarchy of ODEs of second order:

$$
\begin{aligned}
& \mathcal{L}^{*} \eta_{1}=-c_{1} \partial_{\xi} \Phi^{*}, \\
& \mathcal{L}^{*} \eta_{3 / 2}=-c_{3 / 2} \partial_{\xi} \Phi^{*}, \\
& \mathcal{L}^{*} \eta_{2}=-\eta_{1}-c_{1} \partial_{\xi} \eta_{1}-c_{2} \partial_{\xi} \Phi^{*}-\frac{1}{2} f^{\prime \prime}\left(\Phi^{*}\right) \eta_{1}^{2}, \\
& \mathcal{L}^{*} \eta_{5 / 2}=-\frac{3}{2} \eta_{3 / 2}-c_{1} \partial_{\xi} \eta_{3 / 2}-c_{3 / 2} \partial_{\xi} \eta_{1}-c_{5 / 2} \partial_{\xi} \Phi^{*}-f^{\prime \prime}\left(\Phi^{*}\right) \eta_{1} \eta_{3 / 2},
\end{aligned}
$$

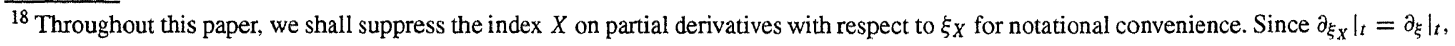
this does not lead to any ambiguities. 
etc. Generally,

$$
\begin{aligned}
\mathcal{L}^{*} \eta_{n / 2}= & -\frac{1}{2}(n-2) \eta_{(n-2) / 2}-\sum_{m=2}^{n-2} c_{m / 2} \partial_{\xi} \eta_{(n-m) / 2}-c_{n / 2} \partial_{\xi} \Phi^{*} \\
& -\left.\sum_{k=2}^{\infty} \frac{f^{(k)}\left(\Phi^{*}\right)}{k !}\left(\sum_{m_{k}} \eta_{m_{k}}\right)^{k}\right|_{\sum_{k} m_{k}=n / 2} \cdot
\end{aligned}
$$

It is important to realize that we do not need to drop nonlinear terms, but that the expansion of $f\left(\Phi^{*}+\eta\right)$ in powers of $\eta$ is also ordered in powers of $1 / \sqrt{t}$. So the higher order terms $\eta^{n}$ find their natural place as inhomogeneities in the equations for $\eta_{i}$ for $i \geq 2$. The hierarchy of ODEs is such that the differential equation for $\eta_{i}$ contains inhomogeneities that depend only on $\eta_{j}$ with $j<i$. The equations therefore can be solved successively. Each $\eta_{i}$ solves a second order differential equation, and the two constants of integration are fixed by the two conditions (3.9) and (3.10). ${ }^{19}$

Note also, that the time dependent collective coordinate $X(t)$ in $\xi_{X}=\xi-X(t)$ only enters Eqs. (3.16)-(3.20) in the form of the constants $c_{n / 2}$, which at this point are still undetermined, and that the functions $\eta_{n / 2}$ obey ODEs.

Let us now compare $\eta=\phi-\Phi^{*}$ to the variations of the profile shape with velocity $v$,

$$
\delta=\Phi_{v^{*}+\dot{X}}-\Phi^{*}=\dot{X} \eta_{\mathrm{sh}}+\frac{1}{2} \dot{X}^{2} \eta_{\mathrm{sh}}^{(2)}+\cdots,
$$

where $\eta_{\mathrm{sh}} \equiv \delta \Phi_{v} /\left.\delta v\right|_{v^{*}}$ is a "shape mode", which gives the change in the profile under a change in $v$. By considering variations of $v$ in the ODE for the profile $\Phi_{v}$, we find that $\eta_{\mathrm{sh}}$ and $\eta_{\mathrm{sh}}^{(2)}$ obey

$$
\begin{aligned}
& \mathcal{L}^{*} \eta_{\mathrm{sh}}+\partial_{\xi} \Phi^{*}=0 \\
& \mathcal{L}^{*} \eta_{\mathrm{sh}}^{(2)}+2 \partial_{\xi} \eta_{\mathrm{sh}}+f^{\prime \prime}\left(\Phi^{*}\right)\left(\eta_{\mathrm{sh}}\right)^{2}=0 .
\end{aligned}
$$

Comparing (3.22) and (3.23) with (3.16)-(3.18), we can identify

$$
\begin{aligned}
& \eta_{1}=c_{1} \eta_{\mathrm{sh}}, \\
& \eta_{3 / 2}=c_{3 / 2} \eta_{\mathrm{sh}} \\
& \eta_{2}=c_{2} \eta_{\mathrm{sh}}+\frac{1}{2} c_{1}^{2} \eta_{\mathrm{sh}}^{(2)}+c_{1} \rho,
\end{aligned}
$$

with $\rho$ a correction term, that solves the equation

$$
\mathcal{L}^{*} \rho+\eta_{\text {sh }}=0 .
$$

In these differential equations, $\eta_{\mathrm{sh}}, \eta_{\mathrm{sh}}^{(2)}$ and $\rho$ obey the conditions

$$
\begin{aligned}
& \eta_{\mathrm{sh}}(0)=0, \quad \eta_{\mathrm{sh}}^{(2)}(0)=0, \quad \rho(0)=0, \\
& \eta_{\mathrm{sh}}(-\infty)=0, \quad \eta_{\mathrm{sh}}^{(2)}(-\infty)=0, \quad \rho(-\infty)=0,
\end{aligned}
$$

cf. (3.9) and (3.10).

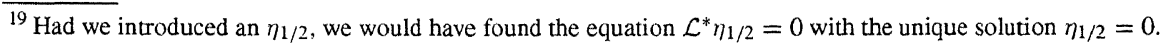


$\rho$ is the first non-vanishing term that indicates the difference between the transient profile $\phi(x, t)$ and the uniformly translating front solution with the instantaneous velocity

$$
v(t)=v^{*}+\dot{X},
$$

as resummation of $\phi$ yields

$$
\phi\left(\xi_{X}, t\right)=\Phi_{v(t)}\left(\xi_{X}\right)+\frac{c_{1}}{t^{2}} \rho\left(\xi_{X}\right)+\frac{t_{0}}{t^{2}} \eta_{\mathrm{sh}}\left(\xi_{X}\right)+\mathrm{O}\left(\frac{1}{t^{5 / 2}}\right) .
$$

This equation confirms that up to order $1 / t^{2}$, the profile shape is given by the solution $\Phi_{v(t)}$ of the ODE with the instantaneous velocity $v(t)$.

Some remarks on these results are in place:

1. We see, that the dynamics in the front interior is slaved to the evolution of $v(t)$ imposed by the leading edge, as we anticipated in Section 3.1.3.

2. The fact that the profile $\phi$ is up to order $t^{-2}$ given by $\Phi_{v(t)}\left(\xi_{X}\right)$ can be traced back to the fact that since $v(t)$ varies as $t^{-1}$, the time derivative term $\partial_{t} \phi$ in the dynamical equation generates terms of order $t^{-2}$. This is why the first two equations in the hierarchy, (3.16) and (3.17), coincide with the ODE (3.22) for the shape mode.

3. Based on numerical data, Powell et al. [67] have conjectured that $\phi$ converges along the trajectory in function space formed by the $\Phi_{v}$ 's with $v<v^{*}$. We here have derived this result analytically, and identify the velocity $v$ of the transients $\Phi_{v}$ with the actual instantaneous velocity $v=v^{*}+\dot{X}$ of the front. We find a non-vanishing correction of order $1 / t^{2}$ to $\phi \approx \Phi_{v^{*}+\dot{X}}$.

4. The transients $\Phi_{v}$ have always $v<v^{*}$ at late times, since we will find that $c_{1}=-\frac{3}{2}$, in accord with the discussion of Section 3.1.1. Note that as discussed in Section 2.2, such $\Phi_{v}$ 's are positive from $\xi_{X} \rightarrow-\infty$ up to a finite value of $\xi_{X}$ only. For the transient (3.31), we need only the positive part of $\Phi_{v}$. The transient (3.31) crosses over to a different functional form, before $\Phi_{v}$ becomes negative.

5. There is a non-universal contribution of order $1 / t^{2}$ to (3.31). It is non-universal, because it depends on initial conditions: the structure of our expansion (3.14) and (3.15) is an asymptotic expansion about $t \rightarrow \infty$, that does not fix $t=0$. We thus can expand in $1 /\left(t-t_{0}\right)=1 / t+t_{0} / t^{2}+\mathrm{O}\left(1 / t^{3}\right)$ just as well as in $1 / t$. This allows us to add an arbitrary multiple of $\eta_{\mathrm{sh}} / t^{2}$ to $\phi$ in Eq. (3.31). The order $1 / t^{2}$ term in (3.31) is thus always non-zero, because the function $\rho\left(\xi_{X}\right)$ in (3.32) is non-vanishing and not a multiple of $\eta_{\mathrm{sh}}\left(\xi_{X}\right)$, but its precise value will depend on initial conditions.

6. The expansion is an asymptotic expansion [100]. Thus, when we have determined the coefficients $c_{1}$ and $c_{3 / 2}$ in (3.14) later, these are the exact prefactors if we expand the velocity and shape in inverse powers of $t$ in the limit $t \rightarrow \infty$. However, the expansion will not have a finite radius of convergence in $1 / \sqrt{t}$.

\subsection{Interior shape expanded towards the leading edge}

We will now see that for $\xi_{X} \geq O(\sqrt{t})$, the structure of our expansion (3.15) breaks down. We then have to resum the terms and use a different expansion. ${ }^{20}$

Let us calculate the contributions $\eta_{i}$ from (3.16)-(3.20) explicitly in the leading edge region, where $\xi_{X} \gg 1$ and $\phi, \Phi^{*} \ll 1$. In this region $\mathcal{L}^{*}(3.13)$ and $\Phi^{*}(2.17)$ are

$$
\mathcal{L}^{*}=\partial_{\xi}^{2}+2 \partial_{\xi}+1, \quad \Phi^{*}=\left(\alpha \xi_{X}+\beta\right) \mathrm{e}^{-\xi_{X}}, \quad \text { for } \xi_{X} \gg 1 .
$$

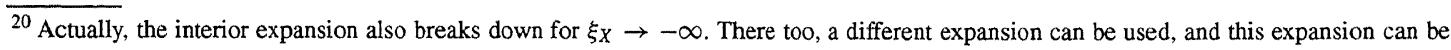
matched to the one we introduced for the interior region. We will not discuss this further here, as it is of no further consequence.
} 
We remove the exponential through the transformation

$$
\mathcal{L}^{*}=\mathrm{e}^{-\xi_{X}} \partial_{\xi}^{2} \mathrm{e}^{\xi_{X}}, \quad \eta_{n / 2}=\mathrm{e}^{-\xi_{X}} \psi_{n / 2}, \quad \phi=\mathrm{e}^{-\xi_{X}} \psi .
$$

The differential equations determining the $\psi_{n / 2}$ are explicitly

$$
\begin{aligned}
& \partial_{\xi}^{2} \psi_{1}=c_{1}\left(\alpha \xi_{X}+\gamma\right), \quad \gamma=\beta-\alpha \\
& \partial_{\xi}^{2} \psi_{3 / 2}=c_{3 / 2}\left(\alpha \xi_{X}+\gamma\right) \\
& \partial_{\xi}^{2} \psi_{2}=\left[-1+c_{1}\left(1-\partial_{\xi}\right)\right] \psi_{1}+c_{2}\left(\alpha \xi_{X}+\gamma\right), \\
& \partial_{\xi}^{2} \psi_{5 / 2}=\left[-\frac{3}{2}+c_{1}\left(1-\partial_{\xi}\right)\right] \psi_{3 / 2}+c_{3 / 2}\left(1-\partial_{\xi}\right) \psi_{1}+c_{5 / 2}\left(\alpha \xi_{X}+\gamma\right), \ldots
\end{aligned}
$$

Generally,

$$
\partial_{\xi}^{2} \psi_{n / 2}=\left[-\frac{1}{2}(n-2)+c_{1}\left(1-\partial_{\xi}\right)\right] \psi_{(n-2) / 2}+\sum_{m=3}^{n-2} c_{m / 2}\left(1-\partial_{\xi}\right) \psi_{(n-m) / 2}+c_{n / 2}\left(\alpha \xi_{X}+\gamma\right),
$$

where we have omitted exponentially small corrections of order $e^{-\xi x}$ in the inhomogeneities on the RHS of the equations. The conditions (3.9) and (3.10) on $\eta$ do not influence the solution in the leading edge.

Eqs. (3.34) are easily solved. For $\psi=\mathrm{e}^{\xi_{X}} \phi$, we find in the region $\xi_{X} \gg 1$

$$
\begin{aligned}
\psi= & \mathrm{e}^{\xi_{X}} \Phi^{*}+\sum_{n=2}^{\infty} \frac{\psi_{n / 2}}{t^{n / 2}}=\alpha \xi_{X}+\beta+\frac{c_{1} \alpha \xi_{X}^{3}}{3 ! t}+\frac{c_{1} \gamma \xi_{X}^{2}}{2 ! t}+\mathrm{O}\left(\frac{\xi_{X}}{t}\right)+\frac{c_{3 / 2} \alpha \xi_{X}^{3}}{3 ! t^{3 / 2}}+\mathrm{O}\left(\frac{\xi_{X}^{2}}{t^{3 / 2}}\right) \\
& +\frac{c_{1}\left(c_{1}-1\right) \alpha \xi_{X}^{5}}{5 ! t^{2}}+\frac{c_{1}\left[\left(c_{1}-1\right) \gamma-c_{1} \alpha\right] \xi_{X}^{4}}{4 ! t^{2}}+\cdots+\frac{c_{3 / 2}\left(2 c_{1}-\frac{3}{2}\right) \alpha \xi_{X}^{5}}{5 ! t^{5 / 2}}+\cdots \\
& +\frac{c_{1}\left(c_{1}-1\right)\left(c_{1}-2\right) \alpha \xi_{X}^{7}}{7 ! t^{3}}+\cdots .
\end{aligned}
$$

Obviously, for $\xi_{X} \geq \sqrt{t}$, the expansion is not properly ordered in powers of $1 / \sqrt{t}$ anymore, since, e.g., $\xi_{X}^{3} / t$ eventually will become larger than $\xi_{X}$. A quick inspection of (3.35) shows that we can continue to work in an $1 / \sqrt{t}$ expansion if we use the variable $z=\xi_{X}^{2} / 4 t$ (3.8) instead of $\xi_{X}$. The expression (3.35) can be identified with

$$
\begin{aligned}
\psi= & \sqrt{t} \alpha\left((4 z)^{1 / 2}+\frac{c_{1}(4 z)^{3 / 2}}{3 !}+\frac{c_{1}\left(c_{1}-1\right)(4 z)^{5 / 2}}{5 !}+\frac{c_{1}\left(c_{1}-1\right)\left(c_{1}-2\right)(4 z)^{7 / 2}}{7 !}+\cdots\right) \\
& +t^{0}\left(\beta+\frac{c_{1}(\beta-\alpha)(4 z)}{2 !}+\frac{c_{3 / 2} \alpha(4 z)^{3 / 2}}{3 !}+\cdots\right)+\mathrm{O}(1 / \sqrt{t}) .
\end{aligned}
$$

This resummed expansion anticipates the crossover to the expansion in $z$ and $1 / \sqrt{t}$ below for $\xi_{X}, t \gg 1$, $z=\xi_{X}^{2} / 4 t=\mathrm{O}(1)$, which will fix the coefficients $c_{1}$, etc. Note that for $c_{1}=-\frac{3}{2}$, terms of order $\sqrt{t}$ sum up to $\alpha(4 z t)^{1 / 2} \mathrm{e}^{-z}=\xi_{X} \exp \left\{-\xi_{X}^{2} /(4 t)\right\}$, which is, in dominant order, the behavior already anticipated in Section 3.1.1.

Instead of resumming the interior expansion explicitly, it is much more transparent to write an expansion directly in terms of powers of $1 / \sqrt{t}$ and the similarity variable $z$ of the diffusion equation. This approach, which amounts to a matching procedure, is the subject of the next subsection. 


\subsection{Analysis of the leading edge}

We now take up the analysis of the leading edge region $\xi_{X} \geq O(\sqrt{t})$ in the case that the initial conditions are sufficiently steep, so that for $\psi=\phi \mathrm{e}^{\xi_{X}}$

$$
\lim _{\xi_{X} \rightarrow \infty} \psi\left(\xi_{X}, t\right)<\mathrm{e}^{-\delta \xi_{X}}, \quad \delta>0 .
$$

Note that according to the discussion of Section 2.5, this condition holds at any finite time $t<\infty$ if it is obeyed initially at $t=0$.

We have already argued in Sections 2.5 and 3.1.1 that the asymptotic profile of the leading edge might be expected to be somewhat like a Gaussian in $\xi_{X}$ and $t$ times a Hermite polynomial. Also the resummation of the interior front solution suggests such a form for large $\xi_{X}$. We now investigate this expansion more systematically, and will show that it actually takes the form of a Gaussian times a generalization of Hermite polynomials, namely confluent hypergeometric functions [98].

In passing, we stress that the arguments from 3.1.1 can be compared directly to our calculation here only to lowest order, because we now work with the coordinate $z=(x-2 t-X(t))^{2} /(4 t)$, while we presented our earlier intuitive arguments in the coordinate $z^{*}=(x-2 t)^{2} /(4 t)$. Of course, one can also set up a systematic expansion in the latter coordinate $z^{*}$, but this requires the introduction of logarithmic terms for a proper matching to the interior part of the front. Working throughout in the shifted frames $\xi_{X}=x-2 t-X(t)$ or $z$ avoids this altogether.

In the coordinates $\xi_{X}$ and $t$, the equation of motion for $\psi$ in the leading edge region is (recall that the earlier leading edge representation in (2.49) was in the frame $\xi=x-2 t$ )

$$
\partial_{t} \psi=\partial_{\xi}^{2} \psi+\dot{X}\left(\partial_{\xi}-1\right) \psi+o\left(e^{-\xi X}\right) .
$$

The differential operators transform under change of coordinates to $z=\xi_{X}^{2} / 4 t$ and $t$ as

$$
\left.\partial_{t}\right|_{\xi}=\left.\partial_{t}\right|_{z}-\left.\frac{z}{t} \partial_{z}\right|_{t},\left.\quad \partial_{\xi}\right|_{t}=\left.\left(\frac{z}{t}\right)^{1 / 2} \partial_{z}\right|_{t} .
$$

Motivated by the form (2.45) and the discussion of Section 3.1.1, we extract the Gaussian $\exp \left\{-\xi_{X}^{2} /(4 t)\right\}=\exp \{-z\}$ from $\psi$ by writing:

$$
\psi(\xi, t)=\mathrm{e}^{-z} G(z, t), \quad z=\frac{\xi_{X}^{2}}{4 t} .
$$

This extraction also allows us to make contact later with functions tabulated in [98]. The dynamical equation (3.38) is equivalent to the equation for $G$ :

$$
\left[z \partial_{z}^{2}+\left(\frac{1}{2}-z\right) \partial_{z}-\frac{1}{2}-t \partial_{t}-c_{1}\right] G=\left[\left(\dot{X} t-c_{1}\right)+\dot{X} \sqrt{t} \sqrt{z}\left(1-\partial_{z}\right)\right] G
$$

The equation is organized such that the differential operators of order $t^{0}$ are on the LHS of the equation, while the RHS has the operators of order $t^{-1 / 2}$ and smaller.

In analogy to our earlier expansion (3.15), we now make an ansatz for $G$ in powers of $1 / \sqrt{t}$ times functions of $z$. A glimpse at the form of the interior shape expanded towards the leading edge (3.36) tells us, that the expansion should start with the order $\sqrt{t}$. We write

$$
G(z, t)=\sqrt{t} g_{-1 / 2}(z)+g_{0}(z)+\frac{g_{1 / 2}(z)}{\sqrt{t}}+\cdots .
$$

Insertion of this ansatz into (3.41) again results in a hierarchy of ordinary differential equations, that can be solved successively: 


$$
\begin{aligned}
& {\left[z \partial_{z}^{2}+\left(\frac{1}{2}-z\right) \partial_{z}-1-c_{1}\right] g_{-1 / 2}=0,} \\
& {\left[z \partial_{z}^{2}+\left(\frac{1}{2}-z\right) \partial_{z}-\frac{1}{2}-c_{1}\right] g_{0}=\left[c_{3 / 2}+c_{1} \sqrt{z}\left(1-\partial_{z}\right)\right] g_{-1 / 2},} \\
& {\left[z \partial_{z}^{2}+\left(\frac{1}{2}-z\right) \partial_{z}-c_{1}\right] g_{1 / 2}=\left[c_{2}+c_{3 / 2} \sqrt{z}\left(1-\partial_{z}\right)\right] g_{-1 / 2}+\left[c_{3 / 2}+c_{1} \sqrt{z}\left(1-\partial_{z}\right)\right] g_{0},}
\end{aligned}
$$

$\therefore$ The general solution of the homogeneous equations with two constants of integration $k_{n / 2}$ and $l_{n / 2}$ can be found [98], they are confluent hypergeometric functions. The special solutions $g_{n / 2}^{\mathrm{sp}}$ of the inhomogeneous equations $\mathrm{n}$ also generally be expressed in terms of double integrals over known functions, as discussed in Appendix $\mathrm{F}$. :low we will, however, just guess the series expansion of the special function $g_{0}^{\text {sp }}$ we need. We write the general lution as

$$
g_{n / 2}(z)=g_{n / 2}^{\mathrm{sp}}(z)+k_{n / 2} M\left(c_{1}+\frac{1}{2}(1-n), \frac{1}{2}, z\right)+l_{n / 2} \sqrt{z} M\left(c_{1}+\frac{1}{2}(2-n), \frac{3}{2}, z\right),
$$

zere the functions $M(a, b, z)$ can be expressed by the Kummer series [98]

$$
M(a, b, z)=1+\frac{a z}{b}+\frac{a(a+1) z^{2}}{b(b+1) 2 !}+\cdots+\frac{(a)_{n} z^{n}}{(b)_{n} n !}+\cdots, \quad \text { with } \quad(a)_{n}=\prod_{k=1}^{n}(a+k-1)=\frac{\Gamma(a+n)}{\Gamma(a)} .
$$

st as in the integration of the interior shape in Section 3.2, there are two constants of integration to be determined every solution $g_{n / 2}$. In addition, however, the $c_{i}$ are not just parameters of the equations as in Section 3.2 , but zy now have to be determined also. The conditions we use to determine these three constants per equation are now .36) and (3.37) in analogy to the two conditions (3.9) and (3.10) for the $\eta_{n / 2}$ : (i) The solution $g_{n / 2}$ has to agree th the expansion of the interior towards the leading edge (3.36) for $z \ll 1$. Then the coefficients of $z^{0}$ and $\sqrt{z}$ (3.36) determine the constants of integration $k_{n / 2}$ and $l_{n / 2}$. (ii) The transients have to be sufficiently steep in the nse that they obey (3.37) at any finite time $t$. Because of the form the expansions (3.41) and (3.43), we require that ch term $g$ in the expansion diverges for $z \gg 1$ at most as a power law of $z$, not exponentially as $\mathrm{e}^{z}$. In addition, $=\mathrm{e}^{z} G$ should not diverge as $t \rightarrow \infty$, but approach a time-independent limit. This gives another condition on $\geq$ constants of integration, that can be obeyed only for a particular choice of $c_{(n+3) / 2}$. With these choices of the nstants, the small $z$ expansion of $\psi=\mathrm{e}^{-z} G$ from (3.40) and (3.42) becomes identical with the interior shape panded towards the leading edge (3.36).

We will solve the first two equations (3.43) and (3.44) explicitly, since they determine the universal terms of the locity correction $\dot{X}$. In particular, the solution for $g_{-1 / 2}$ (3.43) will connect to our qualitative discussion of the Iding $1 / t$ velocity convergence term (3.5) in Section 3.1.1. Eq. (3.44) will give the universal subleading term ${ }^{21}$ order $1 / t^{-3 / 2}$

Let us now start with the solution of the homogeneous leading order equation (3.43), where $g_{-1 / 2}^{\mathrm{sp}}(z)=0$. The nstants of integration are fixed by (3.36) as $k_{-1 / 2}=0$ and $l_{-1 / 2}=2 \alpha$. Therefore $g_{-1 / 2}(z)$ is after matching to əinterior

$$
g_{-1 / 2}(z)=2 \alpha \sqrt{z} M\left(c_{1}+\frac{3}{2}, \frac{3}{2}, z\right) .
$$

order to analyze, how $c_{1}$ is determined by the matching and the requirement that all transients are exponentially seper for $\xi_{X} \rightarrow \infty$ than the asymptotic profile, we first recall the large $z$ behavior of Kummer functions $M(a, b, z)$ 8]: for positive $b$ each term of the series (3.47) is finite. If $a$ is neither zero nor a negative integer, the series is

Do not confuse the expansion in $1 / \sqrt{t}$ of the velocity in (3.5) or (3.14) with the denominators in (3.2) and (3.3). These powers of $1 / \sqrt{t}$ in $\xi^{*}=x-v^{*} t$ representation are absorbed into the $X(t)$ of $\xi$ in the $\xi$-representation, as sketched in (3.4). 
infinite. For $a$ zero or a negative integer $a=-n$, the series is finite, since all terms from order $z^{n+1}$ on contain the factor $(a+n)=0$, and for $b=\frac{1}{2}$ or $\frac{3}{2}$, these finite polynomials are Hermite polynomials. The large $z$ asymptotics of $M(a, b, z)$ for positive $b$ is

$$
M(a, b, z) \stackrel{z \rightarrow \infty}{\sim} \begin{cases}z^{a-b} \mathrm{e}^{z} \Gamma(b) / \Gamma(a) & \text { for }-a \notin \mathbb{N}_{0}, \\ z^{|a|}(a)_{|a|} /(b)_{|a|}(|a|) ! & \text { for }-a \in \mathbb{N}_{0},\end{cases}
$$

where $\mathbb{N}_{0}$ denotes the non-negative integers. If one inserts (3.49) into (3.48) one finds for $\xi_{X} \gg \sqrt{t}$

$$
\phi \propto \xi_{X} \mathrm{e}^{-\xi_{X}} \begin{cases}\left(\xi_{X}^{2} / t\right)^{c_{1}} & -c_{1}-\frac{3}{2} \notin \mathbb{N}_{0} \\ \left(\xi_{X}^{2} / t\right)^{-c_{1}-3 / 2} \exp \left\{-\xi_{X}^{2} /(4 t)\right\} & -c_{1}-\frac{3}{2} \in \mathbb{N}_{0} .\end{cases}
$$

For $-c_{1}-\frac{3}{2}$ not a Non-negative integer, we see from (3.50) that $\psi\left(\xi_{X}, t\right)$ does not converge exponentially fast to zero, in violation of the condition (3.37). Accordingly, for the so-called sufficiently steep initial conditions that obey (3.37), we conclude that $c_{1}+\frac{3}{2}$ has to be zero or a negative integer. Possible solutions are

$$
\begin{aligned}
& c_{1}=-\frac{3}{2}, \quad g_{-1 / 2}(z)=2 \alpha \sqrt{z}, \\
& c_{1}=-\frac{5}{2}, \quad g_{-1 / 2}(z)=2 \alpha \sqrt{z}\left(1-\frac{2}{3} z\right), \\
& c_{1}=-\frac{7}{2}, \quad g_{-1 / 2}(z)=2 \alpha \sqrt{z}\left(1-\frac{4}{3} z+\frac{4}{15} z^{2}\right),
\end{aligned}
$$

etc., with the $g_{-1 / 2}$ given by Hermite polynomials.

There are two ways to argue, why generically $c_{1}=-\frac{3}{2}$ is the appropriate solution. (a) If the initial condition is always non-negative, e.g., because $\phi$ is a density, the transient may not have nodes, so $c_{1}=-\frac{3}{2}$ is the only possible solution. (b) If one can create a front with nodes whose leading edge after some evolution is the superposition of the solutions in (3.51), the solution with $c_{1}=-\frac{3}{2}$ propagates quickest, so the other contributions will be convected to the back, and the $c_{1}=-\frac{3}{2}$ solution will dominate at large times [72]. This argument coincides with the argument from Section 2.3, that fronts with nodes generically are not attractors for the long time dynamics for the nonlinear diffusion equation (1.1). A similar reasoning for the leading edge region can be developed from the arguments in Section 6.6. Furthermore, we have checked various initial conditions with nodes numerically and we have found that either the node gets stuck behind the evolving front or moves away to $\xi \rightarrow \infty$ with velocity larger than $v^{*}$, leaving in both cases a leading edge of the front behind that develops with $c_{1}=-\frac{3}{2}$. We thus find for initial conditions (3.37) steeper than $\Phi^{*}$ generically

$$
c_{1}=-\frac{3}{2}, \quad g_{-1 / 2}(z)=2 \alpha \sqrt{z}
$$

This solution is identical with the order $\sqrt{t}$ of $\psi \mathrm{e}^{z}$ with $\psi$ from (3.36). For $\phi$, we find in the region $\xi_{X} \gg 1$ linearizable about the unstable state in leading order

$$
\begin{aligned}
& \phi=\alpha \xi_{X} \exp \left\{-\xi_{X}-\xi_{X}^{2} /(4 t)\right\}\left(1+\mathrm{O}\left(\frac{1}{\xi_{X}}\right)+\mathrm{O}\left(\frac{1}{\sqrt{t}}\right)\right), \\
& \xi_{X}=x-v^{*} t+\frac{3}{2} \ln t+\mathrm{O}\left(\frac{1}{\sqrt{t}}\right),
\end{aligned}
$$

consistent with the arguments from Section 3.1.1.

Integration of $g_{0}$ now gives the subleading universal terms, which are $O(1 / \sqrt{t})$ in (3.53) and (3.54). Insertion of (3.52) into (3.44) results in

$$
\left[z \partial_{z}^{2}+\left(\frac{1}{2}-z\right) \partial_{z}+1\right] g_{0}=2 \alpha\left(\frac{3}{4}+c_{3 / 2} \sqrt{z}-\frac{3}{2} z\right) .
$$


We now can follow Appendix F for the general solution of the inhomogeneous equation, or we rather can guess a special solution of the inhomogeneous equation by noting that the function

$$
F_{N}(z)=\sum_{n=N}^{\infty} \frac{(1)_{n-2} z^{n}}{(1 / 2)_{n} n !}
$$

is proportional to a truncated Kummer series $M\left(-1, \frac{1}{2}, z\right)(3.47)$ and solves

$$
\left[z \partial_{z}^{2}+\left(\frac{1}{2}-z\right) \partial_{z}+1\right] F_{N}(z)=\frac{z^{N-1}}{(1 / 2)_{N-1}(N-1)} .
$$

The special solution of the inhomogeneous equation (3.55) is then easily seen to be

$$
g_{0}^{\mathrm{sp}}(z)=2 \alpha\left(\frac{3}{4}+2 c_{3 / 2} \sqrt{z}-\frac{3}{4} F_{2}(z)\right) .
$$

Comparing (3.56) to (3.47) and (3.49), one finds

$$
g_{0}^{\mathrm{sp}}(z) \stackrel{z \rightarrow \infty}{\sim}-\frac{3}{2} \alpha \sqrt{\pi} z^{-3 / 2} \mathrm{e}^{z} .
$$

The general solution (3.46) of (3.55) is thus

$$
\begin{aligned}
g_{0}(z) & =g_{0}^{\mathrm{sp}}(z)+k_{0}(1-2 z)+l_{0} \sqrt{z} M\left(-\frac{1}{2}, \frac{3}{2}, z\right) \stackrel{z \lll 1}{=}\left(\frac{3}{2} \alpha+k_{0}\right)+\left(4 \alpha c_{3 / 2}+l_{0}\right) \sqrt{z}+\mathrm{O}(z) \\
& z \rightarrow \infty-\left(\frac{3}{2} \alpha \sqrt{\pi}+\frac{1}{4} l_{0}\right) z^{-3 / 2} \mathrm{e}^{z},
\end{aligned}
$$

where we have used (3.49) and (3.59) for the large $z$ asymptotics. Compare now the small $z$ expansion (3.60) with (3.36). One obviously has to identify

$$
\frac{3}{2} \alpha+k_{0}=\beta, \quad 4 \alpha c_{3 / 2}+l_{0}=0 .
$$

If $g_{0}$ would decay asymptotically as $z^{-3 / 2} \mathrm{e}^{z}$ for large $z$ (3.61), the subleading contribution of order $1 / \sqrt{t}$ in $\phi(3.53)$ would not decay like a Gaussian $\exp \left\{-\xi_{X}^{2} /(4 t)\right\}$ as the leading order term does, but it would decay algebraically like $\xi_{X}^{-3}(4 t)^{3 / 2}$. This would destroy the ordering of our expansion (3.42) and lead to a divergence of $\psi$ for $t \rightarrow \infty$. Thus the coefficient of the leading order term $z^{-3 / 2} \mathrm{e}^{z}$ in $g_{0}(3.61)$ has to vanish:

$$
\frac{3}{2} \alpha \sqrt{\pi}+\frac{1}{4} l_{0}=0 .
$$

Eqs. (3.62) and (3.63) fix all constants $k_{0}, l_{0}$ and $c_{3 / 2}$. The velocity correction of order $1 / t^{3 / 2}$ is

$$
c_{3 / 2}=\frac{3}{2} \sqrt{\pi},
$$

and the analytic solution for $g_{0}(z)$ is

$$
g_{0}(z)=\beta(1-2 z)+3 \alpha\left(z-\frac{1}{2} F_{2}(z)\right)+6 \alpha \sqrt{\pi z}\left(1-M\left(-\frac{1}{2}, \frac{3}{2}, z\right)\right),
$$

with $\alpha$ and $\beta$ the coefficients of the asymptotic leading edge shape $\Phi^{*}(\xi)=(\alpha \xi+\beta) \mathrm{e}^{-\xi}$ for $\Phi^{*} \ll 1$. Note that the subleading term $\beta$ contributes only the rather trivial $(1-2 z)$ term, while the coefficient of the leading $\alpha$ contains all non-trivial terms. The result (3.64) and (3.65) reproduces the order $t^{0}$ in (3.36) identically.

We summarize the results obtained from the analysis of the leading edge: the appropriate coordinate system is $\xi_{X}=x-v^{*} t-X(t)$, and the universal velocity correction is given by

$$
\dot{X}=-\frac{3}{2 t}\left(1-\left(\frac{\pi}{t}\right)^{1 / 2}\right)+\mathrm{O}\left(\frac{1}{t^{2}}\right) \text {. }
$$


The shape in the leading edge, where $\phi \ll 1$, is given in terms of the variables $\xi_{X}$ and $t$ by

$$
\begin{aligned}
\phi\left(\xi_{X}, t\right) & =\exp \left\{-\xi_{X}-\xi_{X}^{2} /(4 t)\right\} G\left(\frac{\xi_{X}^{2}}{4 t}, t\right) \\
& =\exp \left\{-\xi_{X}-\xi_{X}^{2} /(4 t)\right\}\left(\alpha \xi_{X}+g_{0}\left(\frac{\xi_{X}^{2}}{4 t}\right)+\frac{1}{\sqrt{t}} g_{1 / 2}\left(\frac{\xi_{X}^{2}}{4 t}\right)+\cdots\right),
\end{aligned}
$$

with $g_{0}(z)$ from (3.65).

Eqs. (3.65), (3.66) and (3.67) are the second part of our final result, valid in the leading edge of the front, where $\phi \ll 1$. It complements our earlier result (3.31), valid in the interior of the front, with functions $\kappa_{v}$ from (3.22) and $\rho_{v}$ from (3.27).

\subsection{Summarizing remarks}

Let us end this section by putting these analytical results into perspective:

1. The requirement that the leading edge remains steeper than the asymptotic profile $\Phi^{*}$ at any finite time together with the requirement that it converges to $\Phi^{*}$ as $t \rightarrow \infty$, determines the velocity convergence constants $c_{n / 2}$. These constants are thus determined in the leading edge by the initial conditions. They are just parameters in the equations for the interior (3.16)-(3.20).

2. The leading order velocity correction $c_{1}$ reproduces Bramson's result [74], which he derived through solving the (nonlinear) diffusion equation with probabilistic methods. The universal subdominant $1 / t^{3 / 2}$ is new.

3. According to our discussion in connection with the interior expansion, $g_{1 / 2}$ and $c_{2}$ should be termed non-universal, because the change from $1 / \sqrt{t}$ to $1 /\left(t-t_{0}\right)^{1 / 2}$ in the asymptotic expansion about $t \rightarrow \infty$ changes these terms. As for (3.31), we conclude that at least parts of these terms depend on initial conditions and are therefore non-universal.

4. We stress once more that the full expansion is only asymptotic in $1 / \sqrt{t}$, but that the prefactors of the $1 / t$ and $1 / t^{3 / 2}$ terms are exact.

5. The leading edge expansion is an intermediate asymptotics in $z$ valid for $1 \ll z \ll \sqrt{t}$ or $\sqrt{t} \ll \xi_{X} \ll t$, respectively. Above, we extensively made use of the crossover to the interior expansion for $z \ll 1$. Let us now look into the breakdown for $z \geq O(\sqrt{t})$, i.e., for $\xi_{X} \geq O(t)$. This second breakdown immediately follows from inserting into (3.42) our results $g_{-1 / 2}(z)=\mathrm{O}(\sqrt{z})$ and $g_{0}(z) \geq \mathrm{O}(z)$ (in fact, $g_{0}(z)=\mathrm{O}(z \ln z)$ according to Appendix F). This new crossover actually needs to exist in view of our discussion in Section 2.5.1: the steepness $\lambda$ is conserved for $x \rightarrow \infty$ for all times $t<\infty$. It will retain the information about the precise initial condition. This region of conserved steepness at $\xi_{X}>\mathrm{O}(t)$ crosses over to the universal Gaussian leading edge region for $\xi_{X}<\mathrm{O}(t)$, which determines the universal relaxation behavior as discussed above. The region of conserved steepness $\lambda$ at $\xi_{X}>\mathrm{O}(t)$ has no further consequence for the dynamics, if the initial steepness is $\lambda_{\text {init }}>\lambda^{*}$. It will disappear towards $\xi_{X} \rightarrow \infty$ by outrunning the leading edge region with an approximately constant speed. This scenario is sketched in Fig. 2.

6. Our result is valid in the pulled regime but it does not apply at the bifurcation point from the pulled to the pushed regime. For nonlinearity (1.10) this means, that the analysis applies for $\epsilon>\frac{3}{4}[65]$. Only then $\Phi^{*}(\xi) \propto \xi \mathrm{e}^{-\xi}$, which is one of the essential ingredients of our asymptotic analysis. For $\epsilon<\frac{3}{4}$, the front is pushed, and convergence is exponential, as discussed in Sections 2.3 and 2.4. For $\epsilon=\frac{3}{4}$, precisely at the pushed/pulled transition, $\Phi^{*}(\xi) \propto \mathrm{e}^{-\xi}$. In this case, convergence is still algebraic, but the analysis of this chapter does not apply exactly. The convergence analysis, however, can be set up along the same lines. As shown in Appendix G 
we then get instead of (3.66)

$$
\dot{X}=-\frac{1}{2 t}\left(1-\frac{1}{2}\left(\frac{\pi}{t}\right)^{1 / 2}\right)+\mathrm{O}\left(\frac{1}{t^{2}}\right) .
$$

Note that the factor $\frac{3}{2}$ of the $1 / t$ term is replaced by $\frac{1}{2}$ at the bifurcation point. Along the lines of the arguments of Section 3.1.1 this can be understood simply from the fact that at the bifurcation point the asymptotic behavior of $\Phi^{*}$ is as $\Phi^{*}(\xi) \sim \mathrm{e}^{-\xi}$, not as $\xi \mathrm{e}^{-\xi}$, and hence that the simple Gaussian leading edge solution $\exp \left\{-\xi-\xi^{2} /(4 t)\right\} / \sqrt{t}$ matches to the asymptotic front profile in leading order. However, the velocity relaxation (3.69) at the pushed/pulled transition does contain a universal subleading term of order $1 / t^{3 / 2}$ that is absent in the relaxation of the linear equation (2.46).

7. Up to now we excluded the particular initial conditions $\phi(x, 0) \simeq x^{-v} \mathrm{e}^{-x}$ from our discussion, since they are neither sufficiently steep nor flat according to our definition. It is amusing to see that also such initial conditions can be treated with our approach. For sufficiently steep initial conditions, we discarded the case that $-c_{1}-\frac{3}{2}$ would be different from an integer or zero after Eq. (3.50), because it would violate the exponential bound (3.37) for large $\xi_{X}$. However, for the above particular initial conditions, the asymptotic behavior (3.37) is replaced by $\psi \approx \xi_{X}^{-v}$ for $\xi_{X} \gg 1$. For any $\nu<2$ one concludes immediately from Eq. (3.50) that

$$
\phi(x, 0) \simeq x^{-v} \mathrm{e}^{-x} \Rightarrow v(t)=2-\frac{v+1}{2 t}+\cdots,
$$

a result also derived by Bramson [74]. In other words, in the case in which the initial conditions are intermediate between sufficiently steep and flat, the prefactor $c_{1}$ does depend on the initial conditions and may even change sign, but the relaxation is still power-law like. To get the next order term in the expansion for these special initial conditions, our expansion will probably have to be generalized. We will comment on this in Section 6 .

\section{Simulations of pulled fronts in the nonlinear diffusion equation}

In this section, we present simulation data for fronts in the nonlinear diffusion equation $\partial_{t} \phi=\partial_{x}^{2} \phi+f(\phi)(1.1)$ propagating into the unstable state $\phi=0$, and compare these with our analytical predictions. In particular, we thoroughly investigate fronts with the nonlinearity $f(\phi)=\phi-\phi^{3}$, so that the equation becomes

$$
\partial_{t} \phi=\partial_{x}^{2} \phi+\phi-\phi^{3} \text {. }
$$

This equation forms pulled fronts with $v^{*}=2$ and $\lambda^{*}=1=D$, if the initial conditions are sufficiently steep. As an example of a nonlinearity allowing for both pushed and pulled fronts, we also present data for $f(\phi)=\epsilon \phi+\phi^{3}-\phi^{5}$ for $\epsilon=0.56$ and 0.96 .

As an initial condition, we here always choose

$$
\phi(x, 0)=\frac{1}{1+\exp \left\{\lambda_{\text {init }}\left(x-x_{0}\right)\right\}} \rightarrow \begin{cases}\exp \left\{-\lambda_{\text {init }}\left(x-x_{0}\right)\right\}, & x \rightarrow \infty, \\ 1, & x \rightarrow-\infty .\end{cases}
$$

According to our analytical results, all initial conditions with initial steepness $\lambda^{*}<\lambda_{\text {init }} \leq \infty$ exhibit the same universal relaxation behavior asymptotically as $t \rightarrow \infty$, if the front is pulled. We indeed do find this in our simulations. Below we only present simulations for $\lambda_{\text {init }}=10$.

The section is organized into a discussion of the specific numerical features of pulled fronts (Section 4.1), the presentation of the raw simulation data for nonlinearities $f(\phi)=\phi-\phi^{3}$ and $f(\phi)=\epsilon \phi+\phi^{3}-\phi^{5}$ (Section 4.2), and a detailed comparison of the simulations for (4.1) with the analytical predictions (Section 4.3). 


\subsection{Numerical features specific to pulled fronts}

To integrate a given initial condition $\phi(x, 0)$ forward in time $t$ for a nonlinear diffusion equation, we use a semi-implicit algorithm which is explained in detail in Section 5.6.6 (Eq. (5.114)). When running the program, we have to choose a spatial and temporal discretization $\Delta x$ and $\Delta t$, a system size $0 \leq x \leq L$, and a position $x_{0}$ of the initial condition within the system. Comparing results for different parameters $\Delta x, \Delta t, L$, and $x_{0}$ to each other and to the analytical predictions in the extreme precision of often better than six significant figures, we find two features specific to the particular dynamic mechanism of pulled fronts.

\subsubsection{Effect of finite difference code}

The numerical results of the simulation depend of course on the step sizes $\Delta x$ and $\Delta t$ of the finite difference code. In fact, in Section 5.6.6, we will have collected all analytical tools to calculate the corrections to $v^{*}=2$, $\lambda^{*}=1$ and $D=1$, that depend on the numerical integration scheme and on the parameters $\Delta x$ and $\Delta t$. All data presented here are derived for $\Delta x=0.01=\Delta t$. For a pulled front in a nonlinear diffusion equation solved with a semi-implicit scheme, our analytical prediction (5.116) yields $v^{*}=2.000075, \lambda^{*}=0.999954$, and $D=1.00035$. We will need the accuracy of the data below, when we compare to our analytical relaxation prediction.

\subsubsection{Effect of finite system size}

In contrast to a pushed front, the final $t \rightarrow \infty$ relaxation of a pulled front very sensitively depends on system size $L$ and front position $x_{0}$. This effect is closely related to the pulled mode of propagation and the breakdown of the linear stability analysis. Because the half-infinite space $x \gg 1$ of the leading edge dominates the dynamics, the very long time dynamics of the front is sensitive to the region at $x \gg 1$, even though there $\phi \ll 1$. More precisely, the diffusive spreading of the linear perturbation as in Eq. (3.3) or (3.67) that determines the speed, strongly depends on the boundary conditions at $\xi_{X}=x-v^{*} t-X(t)=\mathrm{O}(\sqrt{4 D t})$.

For this reason, we shift the front back to its original position $x_{0}$ within the system after every time step $t_{2}-t_{1}=1$. This eliminates the $x$-interval $0 \leq x \leq x_{\text {shift }} \approx v^{*}$ on the back side of the front from our data, while a new $x$-interval $L-x_{\text {shift }} \leq x \leq L$ has to be created. One might assume that this procedure yields good results for integration times $T$ up $T=\mathrm{O}\left(\left(L-x_{0}\right)^{2} /(4 D)\right)$ because of the diffusive nature of the spreading. However, the precision noticeably breaks down earlier because of the arbitrariness of the newly created $x$-interval $L-x_{\text {shift }} \leq x \leq L$ in the shift process. Filling this region with the constant $\phi(x)=\phi\left(L-x_{\text {shift }}\right)$ creates a flat initial condition, and the front accelerates beyond $v^{*}$ for sufficiently long times. We therefore use $\phi(x) \equiv 0$ in this region. The observed velocities $v_{\phi}(t)$ for $L<\infty$ then always will stay below those in the infinite system $L \rightarrow \infty$. The simulations in the finite system are close to those in the infinite system up to times $T=\mathrm{O}\left(\left(L-x_{0}\right) / v^{*}\right)$.

\subsection{Simulation data}

\subsection{1. $f(\phi)=\phi-\phi^{3}$ : pulled fronts}

As an example, we will extensively discuss simulations of Eq. (4.1). We present data with initial conditions (4.2) and $\lambda_{\text {init }}=10$, where the initial condition is located at $x_{0}=100$ in a system of size $L=1000$. According to our estimate above, the simulations then should be reliable up to times $t$ of order $\frac{1}{2}\left(L-x_{0}\right)=450$. We present data up to $t=400$. The data from this simulation is evaluated in a sequence of figures showing increasing detail and precision.

Fig. 1 already showed the temporal evolution of a sufficiently steep initial condition under the equation of motion (4.1). It shows both, the total displacement of the front, and the evolution of the front shape. We now choose different presentations that show these two different aspects of the dynamics separately and in higher precision. 

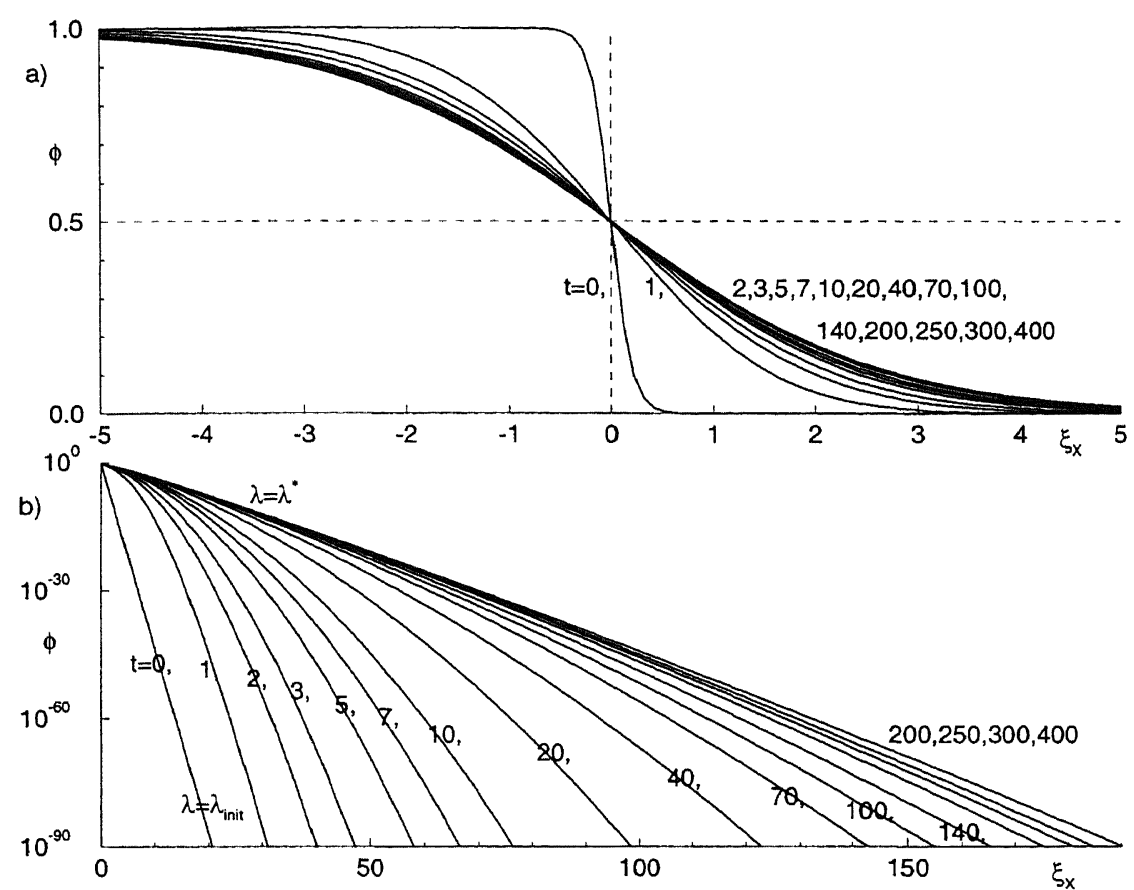

Fig. 4. Simulation of the evolution of the shape of a front under (4.1) at the times denoted in the figure. The initial condition is (4.2) with $\lambda_{\text {init }}=10$. The comoving frame $\xi_{X}$ is chosen in such a way that $\phi\left(\xi_{X}=0, t\right)=\frac{1}{2}$ for all $t$ : (a) a plot of $\phi$ versus $\xi_{X}$ shows mainly the interior of the front; (b) a plot of $\log \phi$ versus $\xi_{X}$ for sufficiently large $\xi_{X}$ shows mainly the leading edge of the front. Note the different scales of $\xi_{X}$.

Let us first study the evolution of the front shape: in Fig. 4, we present $\phi\left(\xi_{X}, t\right)$ as a function of $\xi_{X}$, where $\xi_{X}=x-v^{*} t-X(t)$, Eq. (3.7), is adjusted such that $\phi(0, t)=\frac{1}{2}(3.9)$ for all times $t$. The remaining dynamics in this frame is then the pure evolution of the shape from its steep initial profile $\phi\left(\xi_{X}, 0\right)$ towards its flatter asymptotic profile $\phi \rightarrow \Phi^{*}\left(\xi_{X}\right)$ as $t \rightarrow \infty$. Fig. 4(a) shows $\phi$ as a function of $\xi_{X}$ on the interval $-5<\xi_{X}<5$. One sees the interior or nonlinear part of the front. Fig. 4(b) shows $\log \phi$ in the range $10^{-90}<\phi<1$. This plot is appropriate to show the development of the leading edge, which here essentially determines the dynamics. Accordingly, a very different range of $\xi_{X}$ has to be plotted, namely $0<\xi_{X}<190$. As sketched already in Fig. 2, the leading edge consists of two regions, namely the "Gaussian" region, through which the asymptotic steepness $\lambda^{*}$ spreads in time towards larger $\xi_{X}$, and the region of conserved steepness $\lambda=\lambda_{\text {init }}$ in front of it. In fact, Fig. 4(b) shows that the initial $\lambda_{\text {init }}=10$ on the level $\phi=10^{-90}$ is still fully present for times $t=1$ and 2 , while at later times it gradually approaches $\lambda^{*}=1$. At higher levels, $\phi=10^{-10}$ say, this process of replacement of one steepness by the other is essentially completed at time $t=70$, while at level $10^{-90}$, it is not completed even at time $t=400$, where the simulation stops.

In Fig. 5, we focus on the second feature, namely the displacement of the front. We plot the velocity $v_{\phi}(t)$ of various amplitudes $\phi$ as a function of $t$. According to our previous definition, we identify $v_{1 / 2}(t)=v^{*}+\dot{X}(t)$. For comparison, the predicted asymptotic value $v^{*}$ is plotted as a dashed line. In Fig. 5(a), the non-universal initial transients up to time $t=20$ are shown on the range $0<v<3$. In Fig. 5(b), the velocities are plotted up to time $t=400$ on the velocity interval $1.97<v<2$. One observes,

- that for fixed $t$, the velocity $v_{\phi}(t)$ is the smaller, the larger $\phi$ is. This is an immediate consequence of the fronts becoming flatter in time, cf. Fig. 4 , 

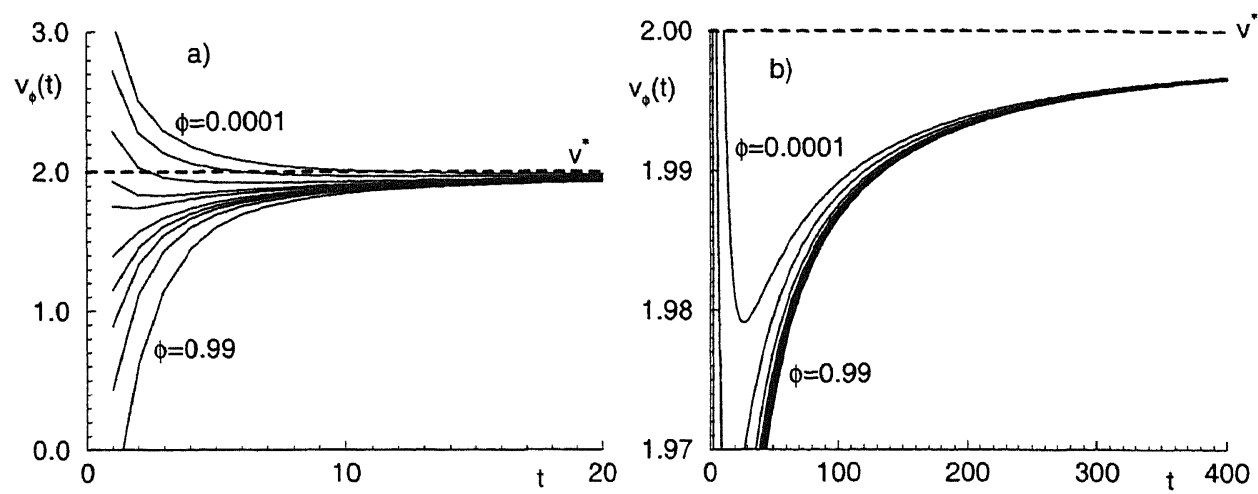

Fig. 5. The same simulation as in Fig. 4. Now the velocities $v_{\phi}(t)$ of amplitudes $\phi=0.99,0.9,0.7,0.5,0.3,0.1,0.05,0.01,0.001$, and 0.0001 (solid lines) are shown as a function of time $t$. The asymptotic velocity $v^{*}$ is marked by the dashed line: (a) initial transients for times $0 \leq t \leq 20$; (b) the same data plotted for longer times $0 \leq t \leq 400$ on an enlarged scale of $v$. The velocities $v_{\phi}(t)$ become largely independent of the "height" $\phi$, and together slowly approach $v^{*}$ in agreement with the predicted universal algebraic relaxation.

- that the $v_{\phi}(t)$ for large $t$ approach a value largely independent of $\phi$, that is still far from the asymptotic value $v^{*}$. We will see below, that this is the signature of the shape relaxing like $v_{\phi_{1}}(t)-v_{\phi_{2}}(t) \propto 1 / t^{2}$ as $t \rightarrow \infty$, while the overall relaxation is $v_{\phi}(t)-v^{*} \propto 1 / t$.

\subsection{2. $f(\phi)=\epsilon \phi+\phi^{3}-\phi^{5}$ : pushed versus pulled fronts}

A well-known example of a nonlinear diffusion equation (1.1) exhibiting both pushed and pulled fronts is given by the nonlinearity (1.10) with $n=2$ :

$$
\partial_{\tau} \varphi=\partial_{y}^{2} \varphi+\epsilon \varphi+\varphi^{3}-\varphi^{5}
$$

This equation for $\epsilon<0$ is often used as a phenomenological (Ginzburg-Landau type) mean field model for a first order transition. Likewise, its extension to a complex field is often used to model a subcritical bifurcation in pattern forming systems. According to arguments recalled in Appendix C, fronts of (4.3) are pushed for $\epsilon<\frac{3}{4}$, and pulled for $\epsilon>\frac{3}{4}$.

The rescaling necessary to bring (4.3) to our standard form (2.2) is discussed in (2.3) and (2.4), and yields

$$
\partial_{t} \phi=\partial_{x}^{2} \phi+\phi+\frac{1}{\bar{\epsilon}} \phi^{3}-\left(1+\frac{1}{\bar{\epsilon}}\right) \phi^{5},
$$

where

$$
\bar{\epsilon}=\frac{1}{2}\left((1+4 \epsilon)^{1 / 2}-1\right), \quad \varphi_{s}^{2}=1+\bar{\epsilon} .
$$

The critical $\bar{\epsilon}$, where the pushed/pulled transition occurs, is $\bar{\epsilon}_{\mathrm{c}}=0.5$.

We present data for the pushed front with $\bar{\epsilon}=0.4(\epsilon=0.56)$ and the pulled front with $\bar{\epsilon}=0.6(\epsilon=0.96)$. The initial condition is the one given before in (4.2). The system size is $L=250$ and the front is located at $x_{0}=50$. The data therefore should be reliable up to time of order 100 , so the data presented extend over $0 \leq t \leq 100$.

In Fig. 6, we plot $v_{\phi}(t)$ as a function of $t$ for both values of $\bar{\epsilon}$ as solid lines, in the same way as the plot of Fig. 6 for the other nonlinearity. The dashed lines denote the asymptotic pulled velocity $v^{*}=2$ predicted for $\bar{\epsilon}=0.6$, and the asymptotic pushed velocity (cf. Appendix C)

$$
v^{\dagger}=\frac{1+4 \bar{\epsilon}}{(3 \bar{\epsilon}(1+\bar{\epsilon}))^{1 / 2}}=2.00594 \text { for } \bar{\epsilon}=0.4
$$




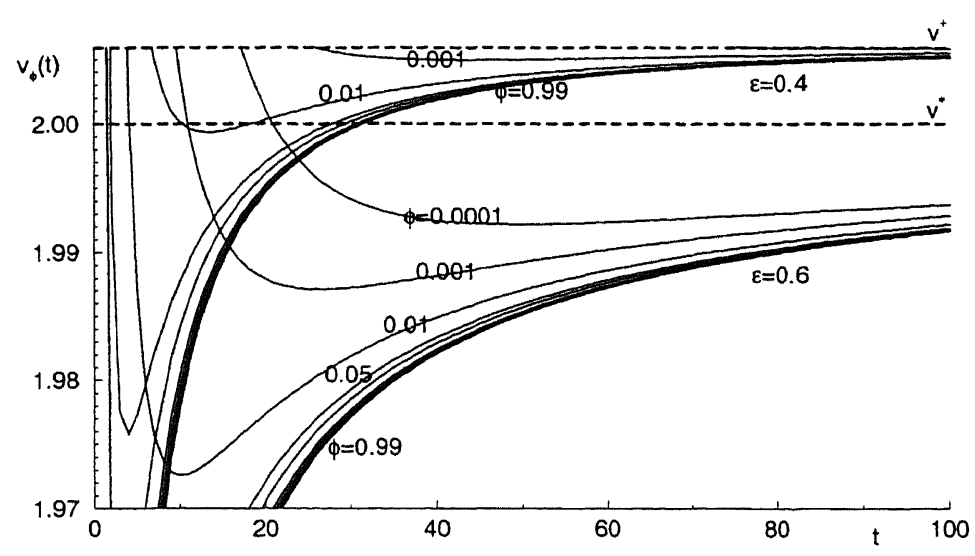

Fig. 6. Plot of $v_{\phi}(t)$ as a function of $t$ as in Fig. 5(b), but now for Eq. (4.3). Simulations for $\bar{\epsilon}=0.4(\epsilon=0.56)$ and $\bar{\epsilon}=0.6(\epsilon=0.96)$ are shown. The dashed lines denote the asymptotic pulled velocity $v^{*}=2$ of the front with $\bar{\epsilon}=0.6$, and the asymptotic pushed velocity $v^{\dagger}=2.00594$ of the front with $\bar{\epsilon}=0.4$. Note the quick exponential relaxation towards $v^{\dagger}$ in contrast to the slow algebraic relaxation towards $v^{*}$. Further away from the transition $\bar{\epsilon}=0.5(\epsilon=0.75)$ from pulled to pushed front propagation, the relaxation in the pushed regime is even faster and the difference $v^{\dagger}-v^{*}$ is larger.

Fig. 6 shows (i) that the simulated fronts in fact do approach the predicted asymptotic velocities, (ii) that up to time $t \leq 10$ both fronts show quite similar initial transients, (iii) that for time $t \gg 10$, however, the relaxation towards the asymptotic velocity $v^{\dagger}$ for $\bar{\epsilon}=0.4$ is much more rapid than that towards $v^{*}$ for $\bar{\epsilon}=0.6$. This very clearly illustrates the difference between pushed exponential and pulled algebraic relaxation, despite the tiny difference between $v^{*}$ and $v^{\dagger}$.

We do not plot the figures of shape relaxation equivalent to Fig. 4, the only difference being that in the pushed case the region of conserved steepness $\lambda=\lambda_{\text {init }}$ at $\xi_{X} \gg 1$ is invaded by a region of steepness $\lambda^{\dagger}$ rather than by the pulled steepness $\lambda^{*} . \lambda^{\dagger}$ is determined by the front interior, while $\lambda^{*}$ is determined by the Gaussian region of the leading edge.

\subsection{Comparison of simulations and analytical predictions}

We now return to our extensive simulation of the pulled front formed by the F-KPP equation $\partial_{t} \phi=\partial_{x}^{2} \phi+\phi-\phi^{3}$, and compare the simulation data to our analytical predictions from Table 2 with $v^{*}=2, \lambda^{*}=1=D$.

\subsubsection{Analysis of the velocity data}

We first concentrate on the analysis of the velocity data $v_{\phi}(t)$ from Fig. 5. The prediction for the velocities $v_{\phi}(t)$ of the amplitudes $\phi$ is derived from the expressions in Table 2 through $v_{\phi}(t)=-\partial_{t} \phi /\left.\partial_{x} \phi\right|_{\phi \text { fixed }}$. The result is

$$
v_{\phi}(t)=v^{*}+\dot{X}-\left.\ddot{X} \frac{\eta_{\mathrm{sh}}}{\partial_{\xi} \Phi^{*}}\right|_{\phi \text { fixed }}+\mathrm{O}\left(\frac{1}{t^{3}}\right) .
$$

Remember, that $\dot{X}$ is universal only till order $1 / t^{3 / 2}$ and will exhibit contributions in order $1 / t^{2}$, that depend on initial conditions. The difference $v_{\phi_{1}}(t)-v_{\phi_{2}}(t)$, however, will turn out to be independent of initial conditions up to order $1 / t^{5 / 2}$. Let us now test these predictions on the simulations in a series of plots with growing precision in Figs. 7-9. 
a) $5<\mathrm{t}<400$

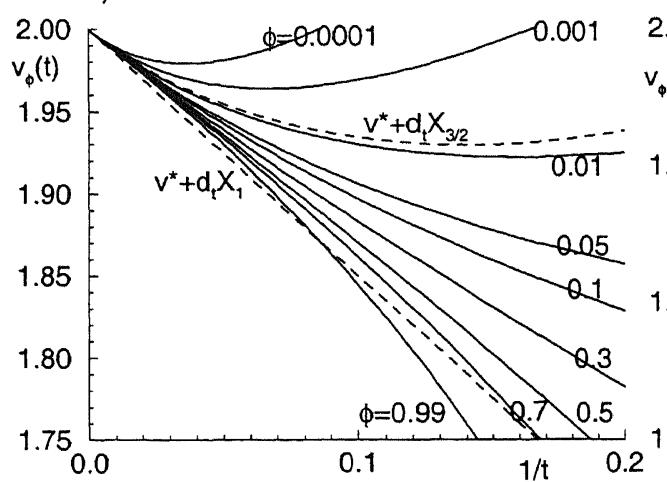

b) $100<\mathrm{t}<400$

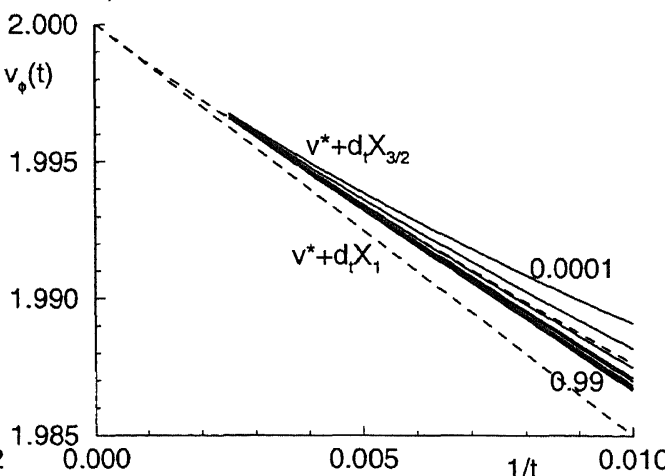

Fig. 7. The data $v_{\phi}(t)$ from Fig. 5, but now plotted over $1 / t$. The lower (straight) dashed line is the asymptote $v(t)=v^{*}+\dot{X}_{1}(t)(4.8)$, the upper (curved) dashed line is the asymptote $v(t)=v^{*}+\dot{X}_{3 / 2}(t)$ with $v^{*}=2$ : (a) time regime $5<t<400$; (b) time regime $100<t<400$. Note that due to the $t^{-3 / 2}$ correction term, the effective slope in this plot is less than $\frac{3}{2}$, even at these long times.

As the velocity correction $\dot{X}$ is $\mathrm{O}(1 / t)$ in leading order, we plot $v_{\phi}(t)$ as a function of $1 / t$ in Fig. 7, for the time range $5<t<400$ in Fig. 7(a), and for $100<t<400$ in Fig. 7(b). The dashed lines present the predicted asymptotes $v^{*}+\dot{X}=2+\dot{X}_{1}(t)$ (the lower dashed line), and $v^{*}+\dot{X}=2+\dot{X}_{3 / 2}(t)$ (the upper-dashed line), where we define

$$
\dot{X}_{1}(t)=-\frac{3}{2 t}, \quad \dot{X}_{3 / 2}(t)=-\frac{3}{2 t}\left(1-\left(\frac{\pi}{t}\right)^{1 / 2}\right) .
$$

First of all, in comparing Figs. 7(a) and (b), we recognize the asymptotic nature of the $1 / \sqrt{t}$ expansion: whether the $\dot{X}_{1}$ or the $\dot{X}_{3 / 2}$ asymptote gives the better prediction, depends on the timescale: if we neglect the upper three
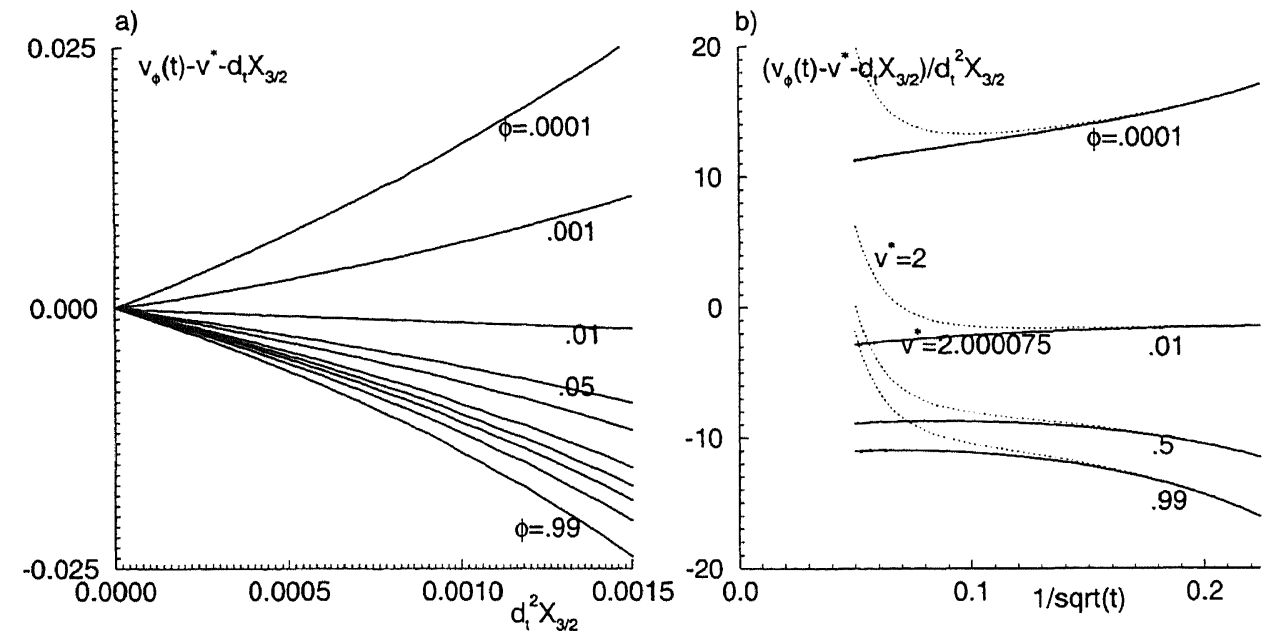

Fig. 8. The data $v_{\phi}(t)$ from Figs. 5 and 7 for times $20 \leq t \leq 400$ in different representations: (a) $v_{\phi}(t)-2-\dot{X}_{3 / 2}$ as a function of $\ddot{X}_{3 / 2}$ (see Eq. (4.8) for the definition of $\left.\dot{X}_{3 / 2}\right)$; (b) $\left(v_{\phi}(t)-v^{*}-\dot{X}_{3 / 2}\right) / \ddot{X}_{3 / 2}$ as a function of $1 / \sqrt{t}$ for $\phi=0.99,0.5,0.01$, and 0.0001 . Dotted lines: $v^{*}=2$, solid lines: with the corrected value $v^{*}=2.000075$ for our numerical scheme and grid size according to Section 5.6.6. 


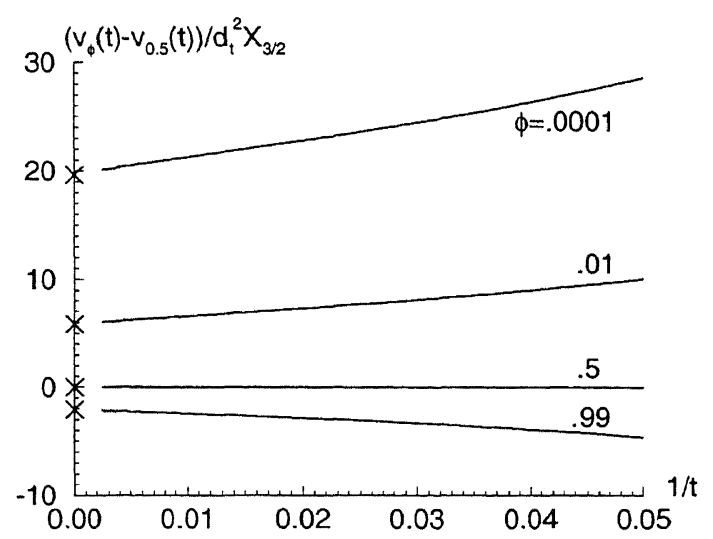

Fig. 9. The solid lines are again the data from Figs. 5,7 and 8 for times $20 \leq t \leq 400$, now plotted as $\left(v_{\phi}(t)-v_{0.5}(t)\right) / \ddot{X}_{3 / 2}$ over $1 / t$ for $\phi=0.99,0.5,0.01$, and 0.0001 . As explained in the text, this eliminates a non-universal $1 / \sqrt{t}$ term that depends on the initial conditions. The crosses result from solving the ODEs for $\Phi^{*}$ and $\eta_{\mathrm{sh}}$ numerically and plotting $-\eta_{\mathrm{sh}} /\left.\partial_{\xi} \Phi^{*}\right|_{\phi}$ for $\phi=0.99,0.5,0.01$, and 0.0001 . Eq. (4.11) predicts that the lines should extrapolate to the crosses. Since they do, and since $\ddot{X}(t)$ is of order $10^{-5}$ at the latest times, these data confirm our predictions with extreme precision.

solid lines with velocities $v_{\phi}(t)$ for the very small amplitudes $\phi=0.01,0.001$, and 0.0001 , the asymptote $2+\dot{X}_{1}$ clearly fits much better in Fig. 7(a) for times $5<t<400$ - while the asymptote $2+\dot{X}_{3 / 2}$ essentially coincides with $v_{0.001}(t)$, an observation we have no analytical explanation for. For times $100<t<400$ in Fig. 7(b), however, the coincidence with $2+\dot{X}_{3 / 2}$ is excellent for all $\phi$, and $2+\dot{X}_{1}$ very clearly is "far off" on this very detailed scale. Hence we will work below with the asymptote $2+\dot{X}_{3 / 2}(t)$, and we present data for the time regime $20<t<400$ in Figs. 8 and 9.

Let us now further zoom in on the $\phi$-dependent velocity corrections (4.7) to $\dot{X}$. Fig. 8(a) shows $v_{\phi}(t)-2-\dot{X}_{3 / 2}$ as a function of $\ddot{X}_{3 / 2}=3 /\left(2 t^{2}\right)\left(1-\frac{3}{2}(\pi / t)^{1 / 2}\right)$. According to the prediction (4.7), the plot for small values of $\ddot{X}_{3 / 2} \rightarrow 0$ should show essentially straight $\phi$-dependent lines, all approaching $v_{\phi}(t)-2-\dot{X}_{3 / 2} \rightarrow 0$ as $\ddot{X}_{3 / 2} \rightarrow 0$. Clearly, that is what they do.

Fig. 8(b) shows one further step of precision aiming now at the precise value of $v^{*}:(4.7)$ predicts

$$
\frac{v_{\phi}(t)-v^{*}-\dot{X}}{\ddot{X}}=-\left.\frac{\eta_{\text {sh }}}{\partial_{\xi} \Phi^{*}}\right|_{\phi \text { fixed }}+\mathrm{O}\left(\frac{g(\phi)}{t}\right) .
$$

However, the evaluation of this expression with $\dot{X}_{3 / 2}$ (4.8) yields $\phi$-independent corrections of order $1 / \sqrt{t}$ :

$$
\frac{v_{\phi}(t)-v^{*}-\dot{X}_{3 / 2}}{\ddot{X}_{3 / 2}}=-\left.\frac{\eta_{\mathrm{sh}}}{\partial_{\xi} \Phi^{*}}\right|_{\phi}+\frac{2 c_{2}}{3}+\frac{3 c_{2} \sqrt{\pi}+2 c_{5 / 2}}{3 \sqrt{t}}+\mathrm{O}\left(\frac{\bar{g}(\phi)}{t}\right) .
$$

Remember, that the constants $c_{2}, c_{5 / 2}$, etc. depend on the initial conditions. According to (4.10), if we plot $\left(v_{\phi}(t)-\right.$ $\left.v^{*}-\dot{X}_{3 / 2}\right) / \ddot{X}_{3 / 2}$ as a function of $1 / \sqrt{t}$, we expect these functions to approach a $\phi$-dependent constant as $1 / \sqrt{t} \rightarrow 0$.

Fig. 8(b) shows, that they in fact do so - but only if we choose the correct value of $v^{*}$ ! The dotted lines show the function for $v^{*}=2$, the fat solid lines for $v^{*}=2.000075$. The latter value is the analytical prediction of $v^{*}$ taking the finite grid size corrections of the numerical code into account, as explained in Sections 4.1.1 and 5.6.6. The two values of $v^{*}$ differ in the sixth significant figure. Fig. 8(b) thus is an extremely precise demonstration of the correctness of our analytical arguments from both Sections 3 and 5, since it clearly confirms our predictions to more than six significant figures! 
Our test in Fig. 8(b) is so sensitive, because we divide in Fig. 8(b) by the small quantities $\ddot{X}_{3 / 2}$, which are of order $10^{-5}$. Without this division the difference of the $v^{*}$ 's in Fig. 8(a) is not yet visible. The plot in Fig. 8(b) shows that we fully understand the specific numerical features of pulled front solutions, both the effect of the finite difference code and of the finite system size, cf. Section 4.1.

We can eliminate $v^{*}$ and the non-universal corrections $-c_{2} / t^{2}$, etc. by plotting $\left(v_{\phi}(t)-v_{0.5}(t)\right) / \ddot{X}_{3 / 2}(t)$ as a function of $1 / t$. Now (4.7) predicts

$$
\frac{v_{\phi}(t)-v_{0.5}(t)}{\ddot{X}_{3 / 2}}=-\left.\frac{\eta_{\text {sh }}}{\partial_{\xi} \Phi^{*}}\right|_{\phi}+\mathrm{O}\left(\frac{1}{t}\right) \text {. }
$$

Fig. 9 shows this plot with the solid lines for $\phi=0.99,0.5,0.01$, and 0.0001 . The crosses on the axis are not (!) extrapolated from the curves, but they mark the predicted asymptotes $-\eta_{\mathrm{sh}} /\left.\partial_{\xi} \Phi^{*}\right|_{\phi}$ for $\phi=0.99,0.5,0.01$, and 0.0001 . The necessary data on $\eta_{\mathrm{sh}}(\xi)$ and $\Phi^{*}(\xi)$ are derived from the numerical solution of the appropriate ODEs, and completely independent from the numerical integration of the PDE for the initial value problem. The coincidence of the extrapolated PDE data with the analytically predicted ODE asymptote is most convincing.

\subsubsection{Analysis of the shape data}

We now leave the analysis of the velocity data, and come back to the shape data from Fig. 4 . Table 2 immediately yields

$$
\frac{\phi\left(\xi_{X}, t\right)-\Phi^{*}\left(\xi_{X}\right)}{\dot{X} \eta_{\mathrm{sh}}\left(\xi_{X}\right)}=1+O\left(\frac{1}{t}\right) .
$$

This gives the clue on how to rewrite the shape data $\phi\left(\xi_{X}, t\right)$ at different times as a function of $\xi_{X}$. The solutions of the ODEs for $\eta_{\mathrm{sh}}$ and $\Phi^{*}$ that are needed for evaluating (4.12) are derived numerically. They have been used for generating the crosses in Fig. 9 and are now also used in Fig. 10.

Plotting the LHS of Eq. (4.12) allows us to combine the information about the interior from Fig. 4(a) and the information about the leading edge from Fig. 4(b) into one plot. In Fig. 10(a), we do not divide by $\dot{X}$, but present the data at the small times $t=1,2,3,5,7,10$, and 20 as $-\left(\phi-\Phi^{*}\right) / \eta_{\text {sh }}$ over $\xi_{X}$. In Fig. 10(b), the data at the large
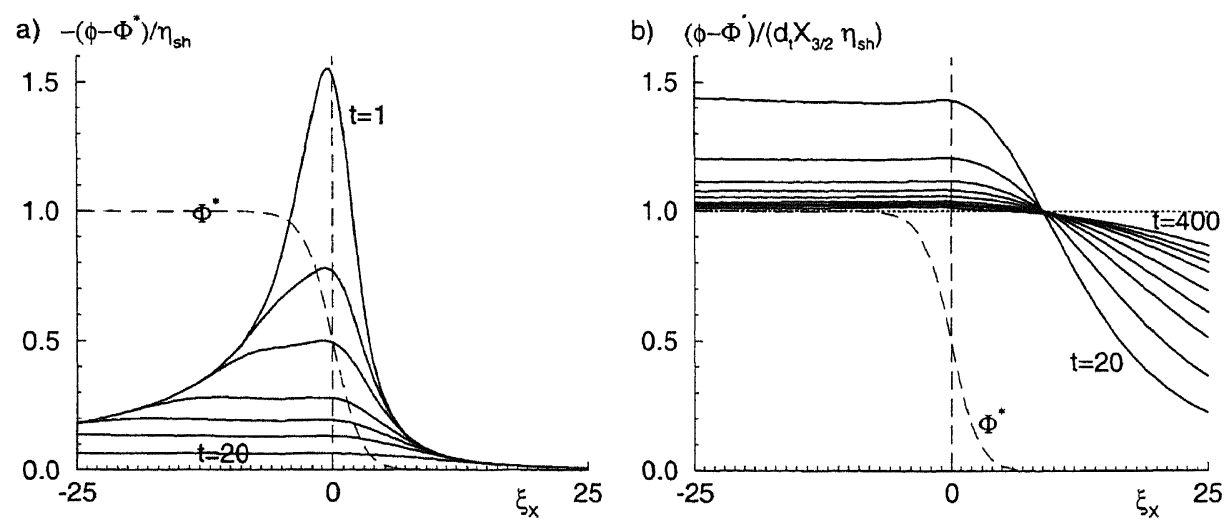

Fig. 10. In this figure, the shape data from Fig. 4 are represented differently using data from the numerical solution of the ODEs for $\Phi^{*}$ and $\eta_{\mathrm{sh}}$ : (a) $-\left(\phi-\Phi^{*}\right) / \eta_{\text {sh }}$ (solid) as a function of $\xi_{X}$ for times $t=1,2,3,5,7,10$, and 20; (b) $\left(\phi-\Phi^{*}\right) /\left(\dot{X}_{3 / 2} \eta_{\text {sh }}\right)$ (solid) as a function of $\xi_{X}$ for times $t=20,40,70,100,140,200,250,300,400$. Dotted line: predicted asymptote $\left(\phi-\Phi^{*}\right) /\left(\dot{X} \eta_{\mathrm{sh}}\right) \rightarrow 1$ as $t \rightarrow \infty$. Dashed lines in (a) and (b) give $\Phi^{*}\left(\xi_{X}\right)$ and $\xi_{X}=0$ for orientation. 
times $t=20,40,70,100,140,200,250,300$, and 400 are shown as $\left(\phi-\Phi^{*}\right) /\left(\dot{X} \eta_{\mathrm{sh}}\right)$ over $\xi_{X}$, where we use again the approximation $\dot{X}=\dot{X}_{3 / 2}$ (4.8). For comparison, both plots also show $\Phi^{*}\left(\xi_{X}\right)$ and the axis $\xi_{X}=0$ as dashed lines. Fig. 10(b) also shows the large time prediction $\left(\phi-\Phi^{*}\right) / \dot{X} \eta_{\text {sh }} \rightarrow 1$ as $t \rightarrow \infty$ as a dotted line.

Fig. 10(a) shows how the interior of the front rapidly relaxes. Fig. 10(b) demonstrates (i) that with $\dot{X}=\dot{X}_{3 / 2}$, we indeed have chosen the correct asymptote, and (ii) how the predicted asymptotic value $\left(\phi-\Phi^{*}\right) /\left(\dot{X} \eta_{\text {sh }}\right) \rightarrow 1$ as $t \rightarrow \infty$ is approached from above in the interior of the front and from below in the leading edge.

Note that in Fig. 10(b) all lines approximately cross one point of height unity far in the leading edge. We have no intuitive or analytical understanding of this observation.

\section{Generalization of pulling to higher order (sets of) equations}

\subsection{Introduction}

In the last 15 years, it has become clear that many of the observations and intuitive notions concerning the behavior of front solutions of the nonlinear diffusion equation (1.1) generalize to higher order equations or systems of coupled PDEs. First of all, taking the spreading velocity $v^{*}$ of a linear perturbation of the unstable state (Eqs. (1.4) and (1.5)) as the generalization of $v^{*}=2 f^{\prime}(0)^{1 / 2}$ for $(1.1)$, we observe that there are numerous examples $[9,12,17,22,61,64-66,116]$ of fronts whose asymptotic velocity approaches the pulled value $v^{*}$ given by (1.5). So there is no doubt that the mechanism of fronts "being pulled along" by the leading edge generalizes to a large class of equations. Second, there is also quite a bit of evidence for the existence of a pushed regime in more complicated equations. In a number of cases, the pushed regime was again found to be related to the existence of a strongly heteroclinic solution with velocity $v^{\dagger}>v^{*}$. An example of a non-monotonic but still uniformly translating pushed front solution in the EFK equation is shown in Fig. 7 of [65]. In the quintic complex Ginzburg-Landau equation, it has turned out to be possible to solve for a strongly heteroclinic front profile exactly, and in numerical simulations it was empirically found that this solution does play the same role in the front selection process as the pushed front $\Phi^{\dagger}$ in the nonlinear diffusion equation [66]. Pushed fronts also emerge in coupled amplitude equations for chaotic domain boundary motion [21]. For extensions of the Swift-Hohenberg equation there are numerical and perturbative indications that both pulled and pushed regimes occur, and that one can tune the front velocity from one regime to the other with one of the nonlinear terms in the equation [65].

Much of our understanding of the above general findings has been intuitive and empirical, or based on conjectures. We shall now show that many of our results for the second order nonlinear diffusion equation generalize to other equations, not only to (sets of) partial differential equations of higher order, but also to other types of equations like difference-differential equations [22,102], or differential equations with memory kernels [103]. We will concentrate here on equations whose relevant front solutions are uniformly translating. For PDEs in this class, essentially the whole classification of nonlinearities and initial conditions $\phi(x, 0)$ in Table 4 applies, provided the uniformly translating fronts $\Phi_{v}$, and in particular the fronts $\Phi^{*}$ and $\Phi^{\dagger}$ exist. Many aspects of the stability analysis can be generalized, while the relaxation of pulled fronts requires the generalization of the calculation in Section 3 . This generalization, that we will develop below, leads to new and explicit predictions for the front convergence in the pulled regime, as summarized in Table 2 . The fact that these predictions for various examples are fully corroborated numerically in Section 5.6 makes us conclude that the velocity selection and relaxation of uniformly translating fronts is now essentially understood even for general sets of equations.

While this paper was nearing completion, it was becoming increasingly clear that even though pattern forming fronts - both fronts leading to regular periodic patterns, as in the Swift-Hohenberg equation [60,65,71], and fronts leading to chaotic patterns as in some parameter ranges of the complex Ginzburg-Landau equation - present additional complications, our most central result for the universal algebraic velocity relaxation carries over even 
to these. We will leave this discussion to the future $[72,73,99]$, and focus here on PDEs whose asymptotic pulled fronts are uniformly translating front solutions of the type $\Phi^{*}\left(x-v^{*} t\right)$, just as in the nonlinear diffusion equation. In writing this section, we face the following two dilemmas:

The extension of both the stability considerations of uniformly translating front solutions of Section 2 and of the relaxation analysis of pulled fronts of Section 3 depends quite crucially on two ingredients: first, that the front propagation into unstable states is in the pulled regime, and, second, that there is a family of uniformly translating front solutions around $\Phi^{*}\left(x-v^{*} t\right)$ : only then the relaxation in the front interior can be along the manifold of front solutions according to $\phi(x, t)=\Phi_{v(t)}\left(\xi_{X}\right)+\mathrm{O}\left(1 / t^{2}\right)$. However, to our knowledge there is no general theory concerning the conditions under which fronts are pulled and concerning the multiplicity of front solutions: for particular equations under study or for some restricted classes of equations, one can often convince oneself that the front should be pulled and that $\Phi^{*}$ should be a member of a family of front solutions, but a general theory is lacking.

An immediate jump to the most general (but abstract) case is pedagogically not justified and moreover would assume knowledge of the derivation of the pulled velocity $v^{*}$ that most readers probably do not have.

We have chosen to deal with these dilemmas by simply summarizing our main assumptions and our results concerning the extensions of Section 2 to more general equations below, relegating the details of the analysis to Appendices. Then, we proceed with the relaxation analysis of pulled fronts in two steps. We first consider in Section 5.3 the analysis of a single PDE which is of first order in time but of arbitrary order in space. After that, the extension to PDEs that are of higher order in time is discussed in Section 5.4. The extension to even more general classes of equations, including difference equations or integro-differential equations, e.g., with memory kernels, is then immediate, as we discuss in Section 5.5. We there also discuss coupled equations. Section 5.6 contains the explicit analytical and numerical results for several of the equations listed in Table 1.

\subsection{Basic assumptions underlying the relaxation analysis of pulled fronts; generalization of Table 4}

Most of the results discussed in Section 2 for the nonlinear diffusion equation can be generalized to higher order nonlinear partial differential equations, as well as to difference or integro-differential equations and to coupled equations:

- The family of solutions can be parametrized as well by the steepness $\lambda$ which gives the rate of exponential decay of $\Phi_{v}(\xi)$ as $\xi \rightarrow \infty$.

- If there are one or more strongly heteroclinic solutions, then at each velocity where such a solution exists, there is a strongly heteroclinic mode of the linear stability operator which changes stability, i.e., which is such that the mode is stabilizing for fronts with velocities larger than this value and destabilizing for velocities less than this value. This implies in particular that the pushed velocity $v^{\dagger}$ is the largest velocity at which there is a strongly heteroclinic front solution $\Phi_{v}^{\dagger}$, and that front solutions with $v<v^{\dagger}$ are unstable (see Appendices $\mathrm{H}$ and I).

- The linear spreading velocity $v^{*}$, given by Eqs. (5.16) and (5.17) below, is the pulled front speed and coincides with the minimum of the velocities of uniformly translating fronts $v(\lambda)$ (see Section 5.3.2).

- If there are no strongly heteroclinic solutions with $v>v^{*}$, all front solutions with $v>v^{*}$ are stable to perturbations which are steeper than $\lambda^{*}$, while front solutions with $v<v^{*}$ are unstable: the pulled front solution is then the slowest and steepest solution which is stable.

- The fronts that dynamically emerge from steep initial conditions (falling off faster than $\mathrm{e}^{-\lambda^{*} x}$ ) converge to pulled fronts propagating with speed $v^{*}$.

In this section, we will investigate the front relaxation under the assumptions:

1. The front solutions are pulled, i.e., starting from a steep initial condition the asymptotic front speed $v_{\text {sel }}$ equals the linear spreading speed $v^{*}$ given by Eqs. (5.16) and (5.17) below. 
2. The asymptotic front is uniformly translating, i.e., of the form $\Phi^{*}\left(x-v^{*} t\right)$, and it is a member of a continuous family of uniformly translating solutions $\Phi_{v}(x-v t)$, parametrized by $v$.

To put our general assumptions 1 and 2 into perspective, we note that for a given equation the existence of a family of front solutions can often be demonstrated by counting arguments. This is shown in Appendix H for PDEs of first order in time that are invariant under space reflection. Such counting arguments also lead one to expect that generically either $\Phi^{*}\left(x-v^{*} t\right)$ is a member of a continuous family of front solutions, or there is no uniformly translating front solution $\Phi^{*}$ at all. For, if there is a discrete set of front solutions (solutions $\Phi_{v}$ exist at isolated values of the velocity), there is no particular symmetry reason to have one at $v=v^{*}$, since the existence of an isolated solution depends on the full nonlinear behavior of the ODE, not just on the properties near one of the asymptotic fixed points. We comment in Section 6 on what might happen when there is no uniformly translating front solution, even though the front dynamics is pulled.

\subsection{Pulled front relaxation in single PDEs of first order in time}

In this subsection, we discuss an arbitrary PDE

$$
F\left(\phi, \partial_{x} \phi, \ldots, \partial_{x}^{N} \phi, \partial_{t} \phi\right)=0
$$

for a single field $\phi(x, t)$. We assume that $F$ is analytic in all its arguments, and that the equation admits homogeneous steady-state solutions $\phi=0$ and $\phi=1$. Moreover, we assume $\phi=0$ to be linearly unstable and $\phi=1$ to be linearly stable, and we consider fronts connecting these two asymptotic states as in (2.14). Also, according to our assumption (B), Eq. (5.1) admits a continuous family of uniformly translating fronts $\phi(x, t)=\Phi_{v}(x-v t)$. The linearization of some front $\phi(x, t)$ about some $\Phi_{v}$ generalizes from (2.25)-(2.27) to

$$
\phi(x, t)=\Phi_{v}(\xi)+\eta(\xi, t), \quad \partial_{t} \eta=\mathcal{L}_{v}(\xi) \eta+\mathrm{O}\left(\eta^{2}\right),
$$

where the linear operator is now

$$
\mathcal{L}_{v}(\xi)=\sum_{n=0}^{N} f_{n}(\xi) \partial_{\xi}^{n}+v \partial_{\xi}, \quad f_{n}(\xi)=\frac{-F_{n}(\xi)}{F_{N+1}(\xi)} .
$$

Here the $F_{n}(\xi)$ denote the functional derivatives of $F$ :

$$
F_{n}(\xi)=\left.\frac{\delta F\left(\phi^{(0)}, \ldots, \phi^{(N+1)}\right)}{\delta \phi^{(n)}}\right|_{\phi^{(m)}=\partial_{\xi}^{m} \Phi_{v}(\xi), \quad m<N+1, \quad \phi^{(N+1)}=-v \partial_{\xi} \Phi_{v}(\xi)} .
$$

In order that the $F_{n}$ have no singularities, $F_{N+1}$ should be of one sign; for convenience, we take $F_{N+1}(\xi)<0$ for all $\xi$ and rescale time $t$ such that $F_{N+1}(\infty)=-1$. We also assume that $F_{N}(\xi)$ neither vanishes nor changes sign for any $\xi$.

\subsubsection{The pulled velocity $v^{*}$}

In the pulled regime and with steep initial conditions, the asymptotic front velocity equals the linear spreading velocity $v^{*}$, i.e., the velocity with which a localized perturbation spreads according to the linearized equations. Since the calculation of $v^{*}$ forms the basis of our subsequent analysis, we summarize its derivation in the context of our first order PDE (5.1). The general formulation in Section 5.5, which is necessary to treat difference equations or integro-differential equations, is closest to the original "pinch point" analysis [56,58], from which many of these ideas originally emerged. 
In the rest frame $(x, t)$, the equation linearized about $\phi=0$ is

$$
\partial_{t} \phi=\mathcal{L}_{0}(\infty) \phi=\sum_{n=0}^{N} a_{n} \partial_{x}^{n} \phi
$$

which is the generalization of (2.40), and where we introduced the short-hand notation $a_{n}=f_{n}(\infty)$. The dispersion relation $\omega(k)$ of a Fourier mode $\exp \{\mathrm{i} k x-\mathrm{i} \omega(k) t\}$ is given by

$$
-\mathrm{i} \omega(k)=\sum_{n=0}^{N} a_{n}(\mathrm{i} k)^{n}, \quad \text { for } \phi \sim \exp \{\mathrm{i} k x-\mathrm{i} \omega(k) t\} .
$$

Since we will later again characterize fronts by their exponential spatial decay rate $\lambda=-\mathrm{i} k$, we already define the growth rate $s(\lambda)$ of the steepness $\lambda$ as

$$
s(\lambda)=\operatorname{Re}(-\mathrm{i} \omega(\mathrm{i} \lambda))=\operatorname{Re} \sum_{n=0}^{N} a_{n}(-\lambda)^{n}
$$

for later use. We restrict the analysis to equations where the temporal growth rate $\operatorname{Re}(-\mathrm{i} \omega(k))$ in (5.6) will be negative for short wavelength Fourier modes $k$, i.e., where

$$
\operatorname{Re} a_{N}( \pm \mathrm{i})^{N}<0
$$

since otherwise all smooth solutions will be unstable against perturbations of arbitrarily short wavelengths.

An arbitrary initial condition $\phi(y, 0)$ will develop under (5.5) as

$$
\begin{aligned}
& \phi(x, t)=\int_{-\infty}^{\infty} \mathrm{d} y G(x-y, t) \phi(y, 0), \\
& G(x, t)=\int_{-\infty}^{\infty} \frac{\mathrm{d} k}{2 \pi} \exp \{\mathrm{i} k x-\mathrm{i} \omega(k) t\}
\end{aligned}
$$

in generalization of (2.44)

For sufficiently steep initial conditions $\phi(y, 0)$, the asymptotic behavior of $\phi(x, t)$ can be obtained from the large-time asymptotics of the Green's function $G(5.10)$ that can be evaluated by a saddle point integration [100] (also known as "steepest decent approximation"). The result will depend on the frame of reference. In an arbitrary coordinate system $\xi=x-v t$ with $v$ fixed, a saddle point $k_{n}$ is a saddle of $-\mathrm{i} \omega(k)+\mathrm{i} v k$,

$$
\left.\frac{\mathrm{d}}{\mathrm{d} k}(-\mathrm{i} \omega(k)+\mathrm{i} v k)\right|_{k_{n}}=\left.0 \Rightarrow \frac{\mathrm{d} \omega(k)}{\mathrm{d} k}\right|_{k_{n}}=v .
$$

A polynomial of degree $N(5.6)$ generically has $N-1$ saddle points $k_{n}, n=1, \ldots, N-1,(5.11)$ in the complex $k$-plane. The integral (5.10) is therefore dominated by the saddle point with the largest growth rate through which we can lead the $k$-contour by continuously deforming it off the real axis. If the contour can be deformed to go through several saddle points, the relevant one is thus that particular saddle point $k^{*}(v)$ of the ones we can reach that has the maximal growth rate:

$$
\operatorname{Re}\left(-\mathrm{i} \omega\left(k^{*}\right)+\mathrm{i} v k^{*}\right)=\max _{n} \operatorname{Re}\left(-\mathrm{i} \omega\left(k_{n}\right)+\mathrm{i} v k_{n}\right) .
$$

It will have

$$
D(v)=\left.\frac{1}{2} \frac{\mathrm{d}^{2} \mathrm{i} \omega(k)}{\mathrm{d} k^{2}}\right|_{k^{*}(v)}, \quad \operatorname{Re} D>0
$$


We stress that Eq. (5.11) only expresses the condition for the existence of a saddle point. Which saddle point the $k$-contour can be made to go through by contour deformation is a condition that depends on the global properties of $\omega(k)$ that can only be analyzed for a given dispersion relation. It is not a local condition. For a further discussion of this point we refer to Appendix M: we proceed by assuming that the saddle point indicated by a star $*$ is the one that obeys (5.12) and this condition, without distinguishing this underlying condition with our notation.

The expansion of the integral (5.10) about the saddle point $k^{*}(v)$ can be performed in a frame with arbitrary velocity $v$ and yields

$$
G(x, t)=\exp \left\{\mathrm{i} k^{*} \xi+\left(-\mathrm{i} \omega\left(k^{*}\right)+\mathrm{i} v k^{*}\right) t\right\} \mathcal{I}_{v}(\xi, t), \quad \xi=x-v t .
$$

The integral $\mathcal{I}_{v}(\xi, t)$ is expressed after substitution of $\left(k-k^{*}\right)=\kappa / \sqrt{t}$ as

$$
\mathcal{I}_{v}=\int_{-\infty}^{\infty} \frac{\mathrm{d} \kappa}{2 \pi \sqrt{t}} \exp \left\{\frac{\mathrm{i} \kappa \xi}{\sqrt{t}}-D \kappa^{2}+\mathrm{O}\left(\frac{D_{3} \kappa^{3}}{\sqrt{t}}\right)\right\}=\frac{\exp \left\{-\xi^{2} /(4 D t)\right\}}{(4 \pi D t)^{1 / 2}}\left(1+\mathrm{O}\left(\frac{D_{3} \xi}{D^{2} t}\right)\right)
$$

for large $t$ and arbitrary $\xi$. Obviously $D$ plays the role of a diffusion coefficient. $D_{3}$ is defined below in (5.28).

Generically, the growth (or decay) rate of the saddle point mode $\operatorname{Re}\left(-\mathrm{i} \omega\left(k^{*}(v)\right)+\mathrm{i} v k^{*}(v)\right)$ will be non-vanishing. We now define the particular linear spreading or pulled velocity $v^{*}$ through $\operatorname{Re}\left(-\mathrm{i} \omega\left(k^{*}\right)+\mathrm{i} v^{*} k^{*}\right)=0$, which is equivalent to

$$
v^{*}=\frac{\operatorname{Im} \omega\left(k^{*}\right)}{\operatorname{Im} k^{*}}=\frac{s\left(-\mathrm{i} k^{*}\right)}{\operatorname{Im} k^{*}}, \quad k^{*}=k^{*}\left(v^{*}\right) .
$$

This means, that in the frame moving with velocity $v^{*}$, the absolute value of the Green's function (5.14) neither grows nor decays in leading order. $v^{*}, k^{*}$ and $\omega\left(k^{*}\right)$ are determined by (5.12) and (5.16), and by Eq. (5.11) evaluated at $v^{*}$ :

$$
\left.\frac{\mathrm{d} \omega(k)}{\mathrm{d} k}\right|_{k^{*}}=v^{*}
$$

In addition, the solution determines $D=D\left(v^{*}\right)(5.14)$.

Note that the leading order large $t$ result (5.14) and (5.15) for the Green's function $G$ in (5.9) is diffusive just like in (2.45), despite the fact, that we are dealing here with an equation with higher spatial derivatives. We shall see in Sections 5.4 and 5.5 that this even remains true for much more general types of equations.

Note also that in our discussion of PDEs in this and the next section, we only take the spatial Fourier transform of $G(x, t)$, as in (5.10) above. However, the most general formulation, which also applies to difference equations or integro-differential equations, is most conveniently done by taking a Fourier transform in space and a Laplace transform in time. In the present context, the Green's function $\hat{G}(k, \omega)$ is then defined as

$$
\hat{G}(k, \omega)=\int_{0}^{\infty} \mathrm{d} t \int_{-\infty}^{\infty} \mathrm{d} x \exp \{-\mathrm{i} k x+\mathrm{i} \omega t\} G(x, t)=\frac{1}{S(k, \omega)}, \quad \text { where } S(k, \omega)=\mathrm{i} \omega(k)-\mathrm{i} \omega,
$$

and the long-time asymptotics is determined by the double roots of the characteristic equation $S(k, \omega(k))=0(5.6)$. We defer this type of formulation, which is closer to the "pinch point" analysis of $[56,58]$, to Section 5.5 .

In practice, one first will drop condition (5.12) and generically derive $N$ solutions $\left(k^{*}, v^{*}\right)$ from (5.16) and (5.17) for a given dispersion relation. But as we already pointed out above, not all of these may be appropriate saddle points for the dynamics. Typically there are solutions with $\lambda^{*} \equiv \operatorname{Im} k^{*}>0$ and $v^{*}>0$, which describe a profile spreading to the right and solutions with $\lambda^{*}<0$ and $v^{*}<0$ describing the spreading to the left, and the $k$-contour will have to be deformed through the appropriate one for the left- and right-moving front. These solutions are related by symmetry, if the original PDE is symmetric under space reflection: if (5.6) only contains even powers of $k$ and 
if the $a_{n}$ are real, then for every solution $\left(k^{*}, v^{*}\right)$ there is a solution $\left(-k^{*},-v^{*}\right)$. Moreover, as mentioned already above, there might be various non-trivial saddle point solutions which are not related by symmetry, if the degree $N$ of spatial derivatives is sufficiently large. The saddle point analysis as well as the arguments of Section 2.5.1 for the competition between different solutions of the linearized equations clearly show that the dynamically relevant solution is the one with the largest velocity $v^{*}$ through which the $k$-contour can be led.

However, choosing the saddle point with the largest $v^{*}$ might according to counting arguments (as in Appendix $\mathrm{H}$ ) be inconsistent with assumption 2 from Section 5.2 of the existence of a family of uniformly translating fronts, since one expects the multiplicity of front solutions to be different for every saddle point $\left(v^{*}, k^{*}\right)$. The discussion of this issue we defer to Section 6.4, as for the applications discussed in Section 5.6, this problem does not rise.

\subsubsection{Uniformly translating solutions $\Phi_{v}$}

In the analysis of the nonlinear diffusion equation in Section 2, we saw that the uniformly translating solution $\Phi_{v}$ decayed as $\mathrm{e}^{-\lambda \xi}$ with $\lambda$ real for $v \geq v^{*}$. Here, $\lambda=\lambda_{-}(v)(2.18)$ is the smallest root of $v=s(\lambda) / \lambda$, where $s(\lambda)$ (5.7) here equals $s(\lambda)=\lambda^{2}+1 . v \geq v^{*}$ implied $\operatorname{Re} k=0, \lambda=\operatorname{Im} k>0$. These front solutions were found to be stable to perturbations which are steeper than the front solution $\Phi_{v}$ itself provided there is no pushed front solution $v_{\mathrm{c}}=v^{\dagger}$. The solutions with $v<v^{*}$ had $\operatorname{Re} k \neq 0, \operatorname{Re} \omega \neq 0$, and were unstable.

We will focus here on the immediate generalization of these results, i.e., assume that fronts with $v \geq v^{*}$ have $\operatorname{Re} k=0$, so that their asymptotic spatial decay is as $\mathrm{e}^{-\lambda \xi}$. In particular, this gives for the pulled fronts

$$
\begin{aligned}
\operatorname{Re} k^{*} & =0, \quad \lambda^{*} \equiv \operatorname{Im} k^{*}>0, \quad \operatorname{Re} \omega\left(k^{*}\right)=0, \quad s\left(\lambda^{*}\right) \equiv \operatorname{Im} \omega\left(k^{*}\right)>0, \\
D & =\left.\frac{1}{2} \frac{\mathrm{d}^{2} s}{\mathrm{~d} \lambda^{2}}\right|_{\lambda^{*}}>0, \quad \operatorname{Im} D=0 .
\end{aligned}
$$

With this assumption we consider only the generic case, that dynamically accessible uniformly translating solutions of real equations will be characterized by a real spatial decay rate $\lambda$ and a real growth rate $s$, and that they will leave a homogeneous state $\phi=1$ behind. This might exclude some pathological cases of uniformly translating front solutions, that are not characterized by a real $\lambda .{ }^{22}$

If the saddle point obeys (5.19), the expression for $t \gg 1(5.14)$ and $v=v^{*}$ for the Green's function $G$ reduces to

$$
G(\xi, t)=\exp \left\{-\lambda^{*} \xi\right\} \frac{\exp \left\{-\xi^{2} /(4 D t)\right\}}{\sqrt{4 \pi D t}}\left(1+\mathrm{O}\left(\frac{D_{3} \xi}{D^{2} t}\right)\right), \quad \xi=x-v^{*} t .
$$

Except for a rescaling of time-and length-scales with the real constants $\lambda^{*}$ and $D$, this is precisely the functional form of (2.45).

If we consider the velocity $v(\lambda)$ of the family of front solutions whose asymptotic spatial decay is as $\mathrm{e}^{-\lambda \xi}$ with real $\lambda$, then it is straightforward to see that $\Phi^{*}$ is the slowest of all these uniformly translating fronts: according to the linearized equation (5.5) the solution in the leading edge is as $\exp \{-\lambda x-i \omega(\mathrm{i} \lambda) t\}$. The resulting velocity $v$ is

$$
v(\lambda)=\frac{-\mathrm{i} \omega(\mathrm{i} \lambda)}{\lambda}=\frac{s(\lambda)}{\lambda} \text { for all } \lambda .
$$

\footnotetext{
${ }^{22}$ Elsewhere [99], we will discuss an extension of the notion of uniformly translating fronts that allows to write pattern forming fronts in the Swift-Hohenberg equation as uniformly translating solutions of a suitable set of complex amplitude-like modes. For these we have Re $k \neq 0$. Similar considerations hold for fronts in the complex Ginzburg-Landau equation itself [66].
} 
The minimum of this curve is given by

$$
\begin{aligned}
& 0=\left.\frac{\partial v(\lambda)}{\partial \lambda}\right|_{\lambda^{*}}=\left.\frac{1}{\lambda}\left(\frac{\partial s(\lambda)}{\partial \lambda}-\frac{s(\lambda)}{\lambda}\right)\right|_{\lambda^{*}}, \\
& \left.\frac{\mathrm{d}^{2} v(\lambda)}{\mathrm{d} \lambda^{2}}\right|_{\lambda^{*}}=\frac{2 D}{\lambda^{*}}>0 .
\end{aligned}
$$

Taking into account that $\omega(k)$ is analytic, Eqs. (5.21) and (5.22) are equivalent to Eqs. (5.16) and (5.17), because at a saddle of an analytic function, the maximum as a function of real $k$ coincides with a minimum as a function of imaginary $k$.

The analysis of the stability of the uniformly translating solutions proceeds largely as in Section 2.3: the existence of a family of front solutions implies, according to counting arguments as given in Appendix $\mathrm{H}$, that there is at least a continuous spectrum of eigenmodes of the stability operator. Indeed, if we again write the temporal behavior of the stability eigenmodes as $\mathrm{e}^{-\sigma t}$ and the steepness of the modes as $\Lambda$, and if we first focus on the spectrum of perturbations that is also continuous in $\Lambda$, then we have for the front solutions with $v(\lambda) \geq v^{*}: \sigma=-(s(\Lambda)-v(\lambda) \Lambda)$. Expanding $\Lambda$ of the perturbation about $\lambda$ of the front, we get

$$
\sigma(\Lambda) \approx-\left(\frac{\partial s(\lambda)}{\partial \lambda}-v(\lambda)\right)(\Lambda-\lambda)=-\lambda \frac{\partial v(\lambda)}{\partial \lambda}(\Lambda-\lambda)
$$

using (5.21) for the second identity. Since we showed above that $\partial v / \partial \lambda<0$ for $\lambda<\lambda^{*}\left(v>v^{*}\right), \sigma(\Lambda)>0$ for $\Lambda>\lambda$. This generalizes the result (D.14) for the nonlinear diffusion equation that the front solutions $\Phi_{v}$ are stable to modes from the continuous spectrum which are steeper than the front itself. In addition to the continuous $\Lambda$ spectrum there again may be discrete perturbation modes associated with the existence of pushed front solutions.

We show in Appendix $H$ that the existence of a strongly heteroclinic front solution $\Phi^{\dagger}$ implies the existence of unstable strongly heteroclinic stability modes for $v<v^{\dagger}$, again in parallel to the results for the nonlinear diffusion equation. The central assumption of our further analysis is of course that we are in the pulled regime, and hence that such solutions are absent.

We finally note that the fact that $v(\lambda)$ has a minimum for $\lambda=\lambda^{*}, v=v^{*}$, implies that for $v<v^{*}$ front solutions decay to zero in an oscillatory manner for $\xi \rightarrow \infty$ as they have $\operatorname{Re} k \neq 0$. By expanding the function $v(\lambda)$ about the bifurcation point at $v^{*}, \lambda^{*}$, it is easy to show that for small $\left|v-v^{*}\right|$, this branch of solutions has $\operatorname{Im}\left(k-k^{*}\right)=\lambda-\lambda^{*} \approx\left(\lambda^{*}\right)^{2} v^{\prime \prime \prime} /(12 D)\left|v-v^{*}\right|, \operatorname{Re} k \approx\left(\lambda^{*}\left|v-v^{*}\right| / D\right)^{1 / 2}$, where $v^{\prime \prime \prime}=\left.\left(\partial^{3} v(\lambda) / \partial \lambda^{3}\right)\right|_{\lambda^{*}}$. One usually has $v^{\prime \prime \prime}<0$ and then such solutions are unstable according to a slight generalization of (5.24).

\subsubsection{The leading edge representation}

As in our analysis of the pulled dynamics of the nonlinear diffusion equation, we will find it expedient to study the large time asymptotics in the leading edge by using the leading edge representation $\psi$. For uniformly translating fronts, the immediate generalization of the transformation (2.50) from Section 2 is

$$
\psi(\xi, t)=\phi(x, t) \mathrm{e}^{\lambda^{*} \xi}, \quad \xi=x-v^{*} t
$$

The linearized dynamical evolution equation for the leading edge representation now generalizes (2.49) to

$$
\partial_{t} \psi=\mathcal{D} \psi+o\left(\psi^{2} \mathrm{e}^{-\lambda^{*} \xi}\right)
$$

where

$$
\mathcal{D}=\mathrm{e}^{\lambda^{*} \xi} \mathcal{L}_{v^{*}}(\infty) \mathrm{e}^{-\lambda^{*} \xi}=\sum_{n=2}^{N} D_{n} \partial_{\xi}^{n} .
$$


A short calculation (Appendix $\mathrm{J}$ ) reveals that the constants $D_{n}$ can be expressed in terms of the dispersion relation $\omega(k)(5.6)$ as

$$
D_{n}=\left.\frac{1}{n !} \frac{\partial^{n}}{\partial(-\lambda)^{n}}\left(-\mathrm{i} \omega(\mathrm{i} \lambda)-v^{*} \lambda\right)\right|_{\lambda=\lambda^{*}}=\left.\frac{1}{n !} \frac{\partial^{n}}{\partial(-\lambda)^{n}}\left(s(\lambda)-v^{*} \lambda\right)\right|_{\lambda=\lambda^{*}} .
$$

Note that in this generalized leading edge representation (5.26), the coefficients of $\psi$ and $\partial_{\xi} \psi$ again are vanishing. This is an immediate consequence of the proper choice of $v^{*}$ and $\lambda^{*}$. In fact, for uniformly translating fronts (5.19) $D_{0}=0$ is equivalent to the proper choice of the velocity $v^{*}(5.16)$ and $D_{1}=0$ is equivalent to the saddle point equation (5.17) fixing $\lambda^{*}$ for given $v^{*} . D_{2}$ is obviously identical to $D$ from (5.13). We will see below, that in the leading edge, the contribution proportional to $D_{2}=D$ gives the dominant contribution, while $D_{3}$ appears only in the subdominant term, similar to what we already observed in (5.15). We therefore will essentially recover the results of the nonlinear diffusion equation (1.1), which had the particular property of $D_{n}=0$ for $n>2$.

\subsubsection{The relaxation analysis}

We have now laid the basis for the extension of the analysis of the relaxation of pulled fronts for our more general equation (5.1) in the case of sufficiently steep initial conditions which as before are characterized by the requirement that

$$
\lim _{x \rightarrow \infty} \phi(x, 0) \mathrm{e}^{\lambda x}=0 \text { for some } \lambda>\lambda^{*} .
$$

The analysis in Section 3 for the nonlinear diffusion equation (1.1) was based on the following steps:

Step 1 . The proper choice of the comoving coordinate system

$$
\xi_{X}=x-v^{*} t-X(t), \quad \dot{X}=\frac{c_{1}}{t}+\frac{c_{3 / 2}}{t^{3 / 2}}+\cdots,
$$

allowing for a logarithmic shift $X(t) \propto \ln t$ relative to the asymptotic coordinate system $\xi=x-v^{*} t$.

Step 2. An expansion of $\phi$ in the nonlinear interior part of the front about the asymptotic front profile $\Phi^{*}\left(\xi_{X}\right)$, taken, however, not in the frame moving with velocity $v^{*}$, but in the frame $\xi_{X}=x-\int^{t} \mathrm{~d} t^{\prime} v\left(t^{\prime}\right)$ with velocity $v(t)=v^{*}+\dot{X}(t)$.

Step 3. A resummation of this expansion of $\phi$ in the crossover region towards the leading edge, where the new variable $z=\xi_{X}^{2} /(4 t)$ is introduced for the region with $\xi_{X} \geq \mathrm{O}(\sqrt{t})$.

Step 4. An analysis of the leading edge in variables $z$ and $t$, where $\phi$ is now linearized about the unstable state $\phi=0$, and not about $\Phi^{*}$. The two boundary conditions that $\phi$ crosses over to the functional form of Step 3 for $z \ll 1$, and that $\phi$ is steeper than $\Phi^{*}$ for $z \gg 1$, now determine both the functional form of $\phi$ and the constants $c_{n / 2}$ in $\dot{X}$. (We can think of this as a matching procedure.) In this analysis, the fact that the parameter $\alpha$ in the asymptotics $\Phi^{*}\left(\xi_{X}\right)=\left(\alpha \xi_{X}+\beta\right) \mathrm{e}^{-\lambda^{*} \xi_{X}}$ is non-zero (see Section 2.5.2) plays a central role.

The generalization of these steps to our equation (5.1) which is of higher order in space, is actually quite straightforward. We again use the general coordinate $\xi_{X}(5.30)$ with $\dot{X}(t)$ to be determined. The interior expansion $\eta\left(\xi_{X}, t\right)=\phi-\Phi^{*}\left(\xi_{X}\right)$ from Section 3.2 applies literally, except that we now need to use the linear operator $\mathcal{L}^{*}=\mathcal{L}_{v^{*}}\left(\xi_{X}\right)$ from (5.3). Accordingly, also the resummation (3.31) again is valid, and we again have

$$
\phi=\Phi_{v(t)}\left(\xi_{X}\right)+\mathrm{O}\left(\frac{1}{t^{2}}\right)
$$

with $\Phi_{v}$ a uniformly translating solution of (5.1) with velocity $v$. The correction $\mathrm{O}\left(1 / t^{2}\right)$ is again non-vanishing and non-universal, as it depends on the precise initial conditions. 
The expansion of the interior shape towards the leading edge (3.36) depends on both the differential operator $\mathcal{L}^{*}$ for $\xi \rightarrow \infty$ and on the shape of the asymptotic front $\Phi^{*}$ (3.32). Eq. (3.32) is generalized to

$$
\Phi^{*}(\xi) \stackrel{\xi \gg 1}{\sim}(\alpha \xi+\beta) \mathrm{e}^{-\lambda^{*} \xi}+\cdots
$$

since the saddle point expansion in Section 5.3.2 implies that for a pulled front $\Phi^{*}$, two roots of the dispersion relation coincide. Generally,

$$
\alpha \neq 0
$$

since a calculation resulting in a generalization of (2.54) can be set up along similar lines: if there is a bounded uniformly translating solution $\Phi^{*}(\xi)$, then upon going to the leading edge representation and integrating the equation for $\Psi^{*}(\xi)$ once over $\xi$, we find that $\alpha$ can be expressed in terms of the spatial integral over the nonlinear terms.

How does the leading edge develop under inclusion of the higher spatial derivatives? First of all we observe that the large- $t$-solutions (5.20) and (2.45) of the linearized equation (5.5) are in leading order identical up to rescaling. In other words, the saddle point approximation renders the spreading around the asymptotic exponential solution diffusive. This suggests that the leading edge can be analyzed by the same type of similarity variables $(z, t)$ as in (3.40). In fact, in our shifted coordinate frame $\xi_{X}$ (5.30) the leading edge representation is

$$
\begin{aligned}
& \phi(x, t)=\mathrm{e}^{-\lambda^{*} \xi_{X}} \psi\left(\xi_{X}, t\right), \\
& \partial_{t} \psi=\mathcal{D} \psi+\dot{X}\left(\partial_{\xi}-\lambda^{*}\right) \psi+o\left(\mathrm{e}^{-\lambda^{*} \xi_{X}}\right)
\end{aligned}
$$

with the differential operator $\mathcal{D}$ from Eq. (5.27). After a rescaling with

$$
\begin{aligned}
& \zeta Y=\lambda^{*} \xi_{X}, \quad \tau=D_{2} \lambda^{*^{2}} t, \quad d_{n}=\frac{D_{n} \lambda^{*^{n}}}{D_{2} \lambda^{*^{2}}}, \quad \dot{Y}=\frac{\dot{X} \lambda^{*}}{D_{2} \lambda^{*^{2}}}=\frac{C_{1}}{\tau}+\frac{C_{3 / 2}}{\tau^{3 / 2}}+\cdots, \\
& C_{n}=c_{n} \lambda^{*}\left(D_{2} \lambda^{*^{2}}\right)^{n-1},
\end{aligned}
$$

this equation takes the form

$$
\partial_{\tau} \psi=\left(\partial_{\zeta}^{2}+\sum_{n=3}^{N} d_{n} \partial_{\zeta}^{n}\right) \psi+\dot{Y}\left(\partial_{\zeta}-1\right) \psi
$$

that is the same as Eq. (3.38), except that there are now higher derivatives $\partial_{\zeta}^{n}$. As we show explicitly in Appendix $\mathrm{K}$, the leading edge can be analyzed with the same ansatz as in (3.40) and (3.42),

$$
\begin{aligned}
& \psi\left(\zeta_{Y}, \tau\right)=\mathrm{e}^{-z} G(z, \tau), \quad z=\frac{\zeta_{Y}^{2}}{4 \tau}, \\
& G(z, \tau)=\sqrt{\tau} g_{-1 / 2}(z)+g_{0}(z)+\frac{g_{1 / 2}(z)}{\sqrt{\tau}}+\cdots,
\end{aligned}
$$

and in rescaled variables, one gets

$$
\begin{aligned}
& C_{1}=-\frac{3}{2}, \quad C_{3 / 2}=\frac{3}{2} \sqrt{\pi}, \quad g_{-1 / 2}(z)=2 \alpha \sqrt{z} \\
& g_{0}(z)=\beta(1-2 z)+3 \alpha\left(1+d_{3}\right) z-2 \alpha d_{3} z^{2}-\frac{3}{2} \alpha F_{2}(z)+6 \alpha \sqrt{\pi z}\left(1-M\left(-\frac{1}{2}, \frac{3}{2}, z\right)\right) .
\end{aligned}
$$

In these variables the result is identical with that for the nonlinear diffusion equation in Section 3 , except for the additional terms proportional to $d_{3}$ in $g_{0}(z)$. In particular, the velocity parameters $C_{1}$ and $C_{3 / 2}$ and the leading order 
contribution $g_{-1 / 2}(z)$ are independent of the value of $d_{3}$, just like the subdominant term $\beta$ from (5.32) enters $g_{0}(z)$ but not the other quantities. That for the problem written in variables $z$ and $\tau, d_{3}$ can only contribute in subleading order, is in fact immediately obvious after the transformation. It is surprising, however, that the subleading velocity coefficient $C_{3 / 2}$ is independent of the value of $d_{3}$. We will find it to be unchanged even for much more general equations.

In terms of the unscaled variables, the universal algebraic convergence of the velocity is given by

$$
v(t)=v^{*}-\frac{3}{2 \lambda^{*} t}\left(1-\sqrt{\frac{\pi}{\left(\lambda^{*}\right)^{2} D t}}\right)+\cdots,
$$

where $v^{*}$ and $\lambda^{*}$ are determined by the saddle point equations (5.16) and (5.17) together with (5.12), and where the diffusion coefficient $D(5.13)$ equals $D_{2}$ from (5.28). The central results of this analysis are summarized in Table 2.

\subsection{Generalization to single PDEs of higher order in time}

We now proceed in two further steps of generalization. In this subsection, we first discuss partial differential equations for a single field $\phi(x, t)$, which include higher order temporal derivatives as well as mixed temporal and spatial derivatives. These are of the form

$$
F\left(\phi, \partial_{x} \phi, \ldots, \partial_{x}^{N} \phi, \partial_{t} \phi, \ldots, \partial_{t}^{M} \phi, \partial_{t} \partial_{x} \phi, \ldots, \partial_{t}^{M} \partial_{x}^{N} \phi\right)=0,
$$

generalizing (5.1) to $M \geq 1$. In Section 5.5, we also deal with difference or integro-differential equations and coupled equations.

The extension to equations of type (5.41) presents no conceptual difficulty - we will follow here a route that is the immediate generalization of the discussion in the previous section. The new elements in the discussion will be the fact that higher order temporal derivatives and mixed spatial and temporal derivatives are generated in the dynamical equation for the leading edge representation $\psi$, but as we shall see these turn out not to affect the expression for the velocity relaxation and for the relaxation of the shape in the interior front region. The notation in (5.48)-(5.54), which may strike the reader at first sight as unnecessarily heavy, prepares for the discussion of even more general equations and sets of equations in Section 5.5, where finding a proper scalar leading edge representation is less straightforward than here.

If we linearize (5.41) about $\phi=0$, we get an equation of the form

$$
\sum_{m=0}^{M} \sum_{n=0}^{N} a_{m n} \partial_{t}^{m} \partial_{x}^{n} \phi(x, t)+\mathrm{o}\left(\phi^{2}\right)=0
$$

For solving the initial value problem in time, it is convenient to Fourier-transform in space

$$
\phi(x, t)=\int_{-\infty}^{\infty} \frac{\mathrm{d} k}{2 \pi} \mathrm{e}^{\mathrm{i} k x} \tilde{\phi}(k, t) .
$$

Below we will use the superscript $\sim$ to denote a quantity Fourier transformed in space.

The Fourier transformation of (5.42) results in an ODE of order $M$ for every Fourier mode $\tilde{\phi}(k, t)$ :

$$
\sum_{m=0}^{M} A_{m}(k) \partial_{t}^{m} \tilde{\phi}(k, t)=0, \quad A_{m}(k)=\sum_{n=0}^{N} a_{m n}(\mathrm{i} k)^{n} .
$$

Obviously, we need $M$ functions to specify the initial conditions. We write these as an $M$-dimensional vector:

$$
\tilde{\Phi}=\left(\tilde{\phi}, \partial_{t} \tilde{\phi}, \ldots, \partial_{t}^{M-1} \tilde{\phi}\right)
$$


The equation of motion (5.44) can now be written in Fourier space as

$$
\partial_{t} \underline{\tilde{\phi}}(k, t)=-\underline{\tilde{T}}(k) \cdot \underline{\tilde{\phi}}(k, t)
$$

with the $M \times M$ matrix

$$
\underline{\underline{T}}(k)=\left(\begin{array}{ccccc}
0 & -1 & 0 & \cdots & 0 \\
0 & 0 & -1 & & 0 \\
& \vdots & & \ddots & \\
0 & 0 & 0 & \cdots & -1 \\
A_{0} / A_{M} & A_{1} / A_{M} & A_{2} / A_{M} & \cdots & A_{M-1} / A_{M}
\end{array}\right) .
$$

For later use, we here define the matrix

$$
\underline{\underline{\hat{S}}}(k, \omega)=A_{M}(k)(\underline{\underline{\underline{T}}}(k)-\mathrm{i} \omega \underline{\underline{1}}),
$$

which later will result from a Fourier-Laplace transformation as in (5.18). Here and below, we use the superscript ^ to denote a Fourier-Laplace transformed quantity to distinguish it from spatially Fourier transformed quantities, which are indicated with a tilde. $\underline{\underline{1}}$ is the $M \times M$ identity matrix $1_{m n}=\delta_{m n}$.

The $M$ eigenvalues $\omega_{m}(k)(m=1, \ldots, M)$ of the matrix $\underline{\underline{\tilde{T}}}(k)$ are determined by the characteristic equation $S\left(k, \omega_{m}(k)\right)=0$, where $S(k, \omega)$ is the characteristic polynomial

$$
S(k, \omega)=\operatorname{det} \underline{\underline{\hat{S}}}(k, \omega)=\sum_{m=0}^{M} A_{m}(k)(-\mathrm{i} \omega)^{m}=\sum_{m=0}^{M} \sum_{n=0}^{N} a_{m n}(-\mathrm{i} \omega)^{m}(\mathrm{i} k)^{n} .
$$

Defining the eigenvectors $\underline{\tilde{U}}_{m}(k)$ of the matrix $\underline{\underline{T}}(k)$ through

$$
\underline{\underline{T}}(k) \cdot \underline{\tilde{U}}_{m}(k)=\mathrm{i} \omega_{m}(k) \underline{\tilde{U}}_{m}(k),
$$

and their adjoints through

$$
\underline{\tilde{U}}_{m}^{\dagger}(k) \cdot \underline{\tilde{U}}_{n}(k)=\delta_{m n},
$$

the matrix $\underline{\underline{\tilde{T}}}(k)$ can be written as

$$
\underline{\underline{T}}(k)=\sum_{m=1}^{M} \mathrm{i} \omega_{m}(k) \underline{\tilde{U}}_{m}(k) \times \underline{\tilde{U}}_{m}^{\dagger}(k),
$$

where $x$ denotes the outer product.

Now (5.46) is easily integrated in time and the Fourier transformation inverted. We find in generalization of (5.9) and (5.10):

$$
\begin{aligned}
& \underline{\phi}(x, t)=\int \mathrm{d} y \underline{\underline{G}}(x-y, t) \cdot \underline{\phi}(y, 0), \\
& \underline{\underline{G}}(x, t)=\sum_{m=1}^{M} \int \frac{\mathrm{d} k}{2 \pi} \exp \left\{\mathrm{i} k x-\mathrm{i} \omega_{m}(k) t\right\} \underline{\tilde{U}}_{m}(k) \times \underline{\tilde{U}}_{m}^{\dagger}(k) .
\end{aligned}
$$

Obviously, the quickest growing mode $\underline{\tilde{U}}_{m}(k)$, characterized now by Fourier mode $k$ and branch of solutions $m$, again will be determined by a saddle point (5.16) and (5.17). Even more than in the case of a first-order equation, we 
can in general have more than one saddle point, as each branch of the dispersion relation can in principle have one or more saddle points (a trivial example for two coupled equations is discussed in Appendix L). Again, the relevant saddle point is the one through which the $k$-contour can be deformed and which has the largest velocity $v^{*}$ in the comoving frame. The associated saddle point values are denoted as $k^{*}=\mathrm{i} \lambda^{*}, D$, etc. As before, we assume uniform translation as in (5.19), so that $k^{*}$ and $\omega\left(k^{*}\right)$ are purely imaginary. Suppose that $v^{*}$ lies on the branch $\omega_{1}(k)$. We then find in the comoving frame $\xi=x-v^{*} t$ for long times $t$ :

$$
\underline{\underline{G}}(\xi, t)=\mathrm{e}^{-\lambda^{*} \xi} \frac{\exp \left\{-\xi^{2} /(4 D t)\right\}}{\sqrt{4 \pi D t}} \underline{\tilde{U}}_{1}\left(k^{*}\right) \times \underline{\tilde{U}}_{1}^{\dagger}\left(k^{*}\right)+\cdots
$$

in generalization of (5.20).

This result shows that in the long time limit, the Green's function $\underline{G}$ projects onto the eigendirection $\underline{\tilde{U}}_{1}\left(k^{*}\right)$. The result (5.55) is not restricted to the explicit form (5.47) of the matrix $\underline{T}$, hence it applies to sets of coupled PDEs just as they also can be written in the form (5.46). Projection onto the eigendirection $\tilde{U}_{1}\left(k^{*}\right)$ then defines the scalar leading edge equation resulting from coupled PDEs. We will further exploit this property in the following section.

In this section, we just use (5.55) to calculate $v^{*}$ and $\lambda^{*}$, and to demonstrate why the leading edge transformation catches the relevant dynamics. Proceeding as in earlier sections, the scalar equation (5.42) now transforms under the leading edge transformation with $v^{*}$ and $\lambda^{*}$ to

$$
\begin{aligned}
& \phi(x, t)=\mathrm{e}^{-\lambda^{*} \xi} \psi(\xi, t), \quad \xi=x-v^{*} t, \\
& 0=\sum_{m=0}^{M} \sum_{n=0}^{N} a_{m n}\left(\partial_{t}-v^{*} \partial_{\xi}+v^{*} \lambda^{*}\right)^{m}\left(\partial_{\xi}-\lambda^{*}\right)^{n} \psi=\sum_{m=0}^{M} \sum_{n=0}^{M+N} b_{m n} \partial_{t}^{m} \partial_{\xi}^{n} \psi(\xi, t) .
\end{aligned}
$$

Just as the $a_{m n}$ from Eq. (5.42) can be written in terms of derivatives of the characteristic polynomial $S(k, \omega)$ (5.49) as

$$
a_{m n}=\left.\frac{\left(\mathrm{i} \partial_{\omega}\right)^{m}}{m !} \frac{\left(-\mathrm{i} \partial_{k}\right)^{n}}{n !} S(k, \omega)\right|_{(k=\omega=0)}
$$

so the $b_{m n}$ can be written as derivatives as well, similar to (5.28). In showing this, it simplifies the notation to use coordinates expanded about the saddle point by introducing the variables

$$
\Omega=\omega-v^{*} k, \quad q=k-k^{*}=k-\mathrm{i} \lambda^{*},
$$

and by defining

$$
S^{*}(q, \Omega)=S\left(k^{*}+q, \omega^{*}+v^{*} q+\Omega\right), \quad k^{*}=\mathrm{i} \lambda^{*}, \quad \omega^{*}=v^{*} k^{*} .
$$

When we will later consider the Fourier-Laplace transform of $\psi(\xi, t)$ in the frame $\xi$, the frequency in this frame will turn out to be $\Omega$ and the wave number will turn out to be $q$, since $\exp \{-\mathrm{i} \omega t+\mathrm{i} k x\}=\exp \left\{-\lambda^{*} \xi\right\}(\exp \{-\mathrm{i} \Omega t+\mathrm{i} q \xi\})$. Accordingly, the long-time-small-gradient expansion of $\psi(\xi, t)$ will correspond to a small- $\Omega$-small- $q$ expansion. Indeed, in line with this interpretation, inspection of (5.57) shows that the $b_{m n}$ are simply

$$
b_{m n}=\left.\frac{\left(i \partial_{\omega}\right)^{m}}{m !} \frac{(-\mathrm{i})^{n}\left(\partial_{k}+v^{*} \partial_{\omega}\right)^{n}}{n !} S(k, \omega)\right|_{\left(k^{*}, v^{*} k^{*}\right)}=\left.\frac{\left(\mathrm{i} \partial_{\Omega}\right)^{m}}{m !} \frac{\left(-\mathrm{i} \partial_{q}\right)^{n}}{n !} S^{*}(q, \Omega)\right|_{(q=\Omega=0)} .
$$

We will discuss the precise correspondence between the formulation in terms of $S$ and the dispersion relation $\omega_{1}(k)$ below, and just note here that the saddle point equations that determine $\lambda^{*}$ and $v^{*}$ are expressed by

$$
b_{00}=S^{*}(0,0)=0, \quad b_{01}=-\left.\mathrm{i} \partial_{q} S^{*}(q, \Omega)\right|_{q=\Omega=0}=0 .
$$


After dividing the whole equation (5.57) by $b_{10}$ and introducing the notations

$$
D_{n}=-\frac{b_{0 n}}{b_{10}}, \quad w=\frac{b_{11}}{b_{10}}, \quad \tau_{1}=\frac{b_{20}}{b_{10}}, \quad \text { etc. }
$$

the terms with the lowest derivatives are

$$
\left(\partial_{t}+\tau_{1} \partial_{t}^{2}+\cdots-D_{2} \partial_{\xi}^{2}-D_{3} \partial_{\xi}^{3}+\cdots+w \partial_{t} \partial_{\xi}+\cdots\right) \psi+o\left(\psi^{2} \mathrm{e}^{-\lambda^{*} \xi}\right)=0
$$

This is the leading edge equation in its most general form. Note that after the leading edge transformation, the coefficient $w$ may be non-zero even if the coefficient $a_{11}=0$ of $\partial_{t} \partial_{x} \phi$ in the original equation of motion (5.44) vanishes.

To show the connection with our discussion of first-order equations in earlier sections, it is instructive to analyze the relation between $S$ and the dispersion relation. The various branches $\omega_{m}(k)$ or $\Omega_{m}(q)$ of the dispersion relation are defined implicitly through the roots of

$$
S\left(k, \omega_{m}(k)\right)=0 \Leftrightarrow S^{*}\left(q, \Omega_{m}(q)\right)=0,
$$

As before, let $\omega_{1}(k)\left(\Omega_{1}(q)\right)$ be the branch on which the saddle point determining $v^{*}$ lies. Upon differentiating (5.65) once with respect to $k$ or $q$ and using Eqs. (5.61) and (5.62), we get our familiar result

$$
\left.\frac{\mathrm{d} \omega_{1}(k)}{\mathrm{d} k}\right|_{k^{*}}=\left.v^{*} \Leftrightarrow \frac{\mathrm{d} \Omega_{1}(q)}{\mathrm{d} q}\right|_{q=0}=0 .
$$

Likewise, by differentiating (5.65) twice we get

$$
\left.\frac{\mathrm{d}^{2} \Omega_{1}(q)}{\mathrm{d} q^{2}}\right|_{q=0}=\left.\frac{\mathrm{d}^{2} \omega_{1}(k)}{\mathrm{d} k^{2}}\right|_{k^{*}}=-\left.\frac{\partial_{q}^{2} S(q, \Omega)}{\partial \Omega S(q, \Omega)}\right|_{q=\Omega_{1}(0)=0} .
$$

If we combine this with the expression $D=-b_{02} / b_{10}$, we recover our familiar expression

$$
D=\left.\frac{\partial_{q}^{2} S(q, \Omega)}{2 \mathrm{i} \partial_{\Omega} S(q, \Omega)}\right|_{q=\Omega_{1}(0)=0}=\left.\frac{\mathrm{id}^{2} \Omega_{1}(q)}{2 \mathrm{~d} q^{2}}\right|_{q=0}=\left.\frac{\mathrm{id}^{2} \omega_{1}(k)}{2 \mathrm{~d} k^{2}}\right|_{k^{*}} .
$$

For the case of an equation which is of first order in time, one can easily check that our general expression for $D_{n}$ reduces to the one given before in (5.28), $D_{n}=(-\mathrm{i} / n !) \mathrm{d}^{n} \omega /\left.\mathrm{d}(\mathrm{i} k)^{n}\right|_{k^{*}}$.

Before we discuss the consequences of (5.64), we note in passing that formally we could have proceeded directly from the linearized equation of motion (5.42) to the leading edge representation (5.57) and hence to (5.64), by choosing the two parameters $v^{*}$ and $\lambda^{*}$ such that the two conditions $b_{00}=0=b_{01}$ are obeyed. The detour from this straightforward transformation via the saddle point analysis was taken to bring out the physical origin of the transformation in this context and to show why one has to use the saddle point $\left(v^{*}, \lambda^{*}\right)$ with the largest $v^{*}$ through which the contour can be deformed. In addition, it explicitly shows how a particular "direction" $\underline{U}_{1}\left(k^{*}\right)$ of the vector field $\phi$ corresponds to the slow leading edge dynamics. We will see in the next section that for coupled equations there is some freedom in choosing the projection onto a scalar leading edge variable.

Let us now analyze the implications of the leading edge representation (5.64). First of all, we observe that a uniformly translating pulled front $\Phi^{*}(\xi)=\mathrm{e}^{-\lambda^{*} \xi} \Psi^{*}(\xi)$ still will have the form (5.32) $\Psi^{*}(\xi)=\alpha \xi+\beta$, and that the argument for $\alpha \neq 0$ from Section 2.5 .2 still does apply.

Can the extra terms $\tau_{1} \partial_{t}^{2} \psi, w \partial_{t} \partial_{\xi} \psi$, etc. change our relaxation prediction from Section 5.3? A short inspection shows that after rewriting the equation in variables $z$ and $t$, cf. (5.36)-(5.38) and (3.39), $w \partial_{t} \partial_{\xi} \psi$ will be of the same subleading order in $1 / \sqrt{t}$ as $D_{3} \partial_{\xi}^{3} \psi$, while both the terms $\tau_{1} \partial_{t}^{2} \psi$ and $D_{4} \partial_{\xi}^{4} \psi$ will be one order lower. Also, when 
rewriting the equation in the variable $\xi_{X}=x-v^{*} t-X(t)$, higher temporal derivatives will create terms like $\ddot{X}$ and $\dot{X}^{2}$ from the exponential factor in the leading edge transformation $\phi\left(\xi_{X}, t\right)=\exp \left\{-\lambda^{*} \xi_{X}\right\} \psi\left(\xi_{X}, t\right)$. Since these are of order $1 / t^{2}$, they do not influence the leading and subleading terms.

We do not repeat the detailed calculation here, because it completely follows the lines of the earlier one. One finds that the result again is given by $(5.39)$, except that the subleading $g_{0}(z)$ picks up another polynomial contribution from $w$ besides the one from $D_{3}$, namely

$$
g_{0}(z)=g_{0}(z)[(5.39)]+2 \alpha w \lambda^{*}\left(z^{2}-\frac{3}{4}\right) .
$$

The uniform velocity relaxation is invariably

$$
v(t)=v^{*}-\frac{3}{2 \lambda^{*} t}\left(1-\left(\frac{\pi}{\left(\lambda^{*}\right)^{2} D t}\right)^{1 / 2}\right)+\cdots,
$$

and the interior part of the front is again slaved to the tip like

$$
\phi(x, t)=\Phi_{v(t)}\left(\xi_{X}\right)+\mathrm{O}\left(\frac{1}{t^{2}}\right),
$$

so the predictions from Table 2 also apply to PDEs with higher temporal derivatives like (5.40), if the front is pulled.

Thus we reach the important conclusion that the universal power law convergence is not an artifact of the diffusion-type character of the nonlinear diffusion equation: it holds generally in the pulled regime of uniformly translating fronts, because the expansion about the saddle point, which governs the dynamics of the leading edge representation $\psi$, is essentially diffusive.

\subsection{Further generalizations}

We now complete the last step in our discussion, and show that our results hold much more generally: even if the original dynamical equation is not a PDE, the dynamical equation for the appropriate leading edge variable $\psi$ is still the same diffusion type equation (5.64), and consequently, our results for the velocity and shape relaxation from Table 2 do apply.

When we have a set of coupled equations, we can view them as components of a vector field, using a notation as in (5.46) with a different matrix $\underline{\underline{\underline{T}}}(k)$. The main complication we are facing in this case is that the leading edge dynamics then not only "selects" a velocity $v^{*}$ in the pulled regime, but also an associated eigendirection $\underline{\tilde{U}}_{m}(k)$ in this vector space - this eigendirection determines the relative values of the various fields in the leading edge of the front. The long-time dynamics in the frame moving with the pulled velocity $v^{*}$ is then associated with a slow dynamics along this eigendirection, while the dynamics along the other eigendirections is exponentially damped. The appropriate scalar leading edge variable $\psi$ will then turn out to be nothing but the projection of the dynamics along this slow direction.

The second complication is that we now consider equations whose temporal dependence is not necessarily of differential type $\partial_{t}^{N}$ : they may just as well be of difference type or contain memory kernels. To treat such equations, we also perform a Laplace transformation in time besides the Fourier transformation in space just as in (5.18) by defining

$$
\hat{\phi}_{m}(k, \omega)=\int_{0}^{\infty} \mathrm{d} t \mathrm{e}^{\mathrm{i} \omega t} \tilde{\phi}_{m}(k, t) .
$$

We thus consider dynamical systems that after the Fourier-Laplace-transformation of the equations, linearized about 
the unstable state, are of the form

$$
\sum_{m=1}^{M} \hat{S}_{n m}(k, \omega) \hat{\phi}_{m}(k, \omega)=\sum_{m=1}^{M} \tilde{H}_{n m}(k) \tilde{\phi}_{m}(k, t=0), \quad n=1, \ldots, M .
$$

The terms on the RHS generally arise upon partial integration of temporal derivative terms, when we take the Laplace transform. They contain the initial conditions. Before exploring the implications of (5.73), we first discuss in more detail the type of systems whose linear dynamical equations can be written in the above form.

Sets of PDEs. Single or coupled PDEs can generally be written in the matrix notation $\left(\partial_{t}+\underline{\underline{T}}(k)\right)$. $\underline{\tilde{\phi}}(k, t)=0$ (Eq. (5.44)) and after Laplace transformation immediately yield (5.73), with the matrices $\underline{\underline{S}}(k, \omega)=$ $\bar{A}_{M}(k)(\underline{\tilde{T}}(k)-i \omega \underline{\tilde{1}})$ as before in (5.48), and $\underline{\underline{\tilde{H}}}(k)=A_{M}(k) \underline{\underline{1}}$. The leading edge behavior of single PDEs, where the matrix $\underline{\overline{\tilde{T}}}(k)$ has the explicit form (5.47), was discussed in the previous section. For coupled PDEs, the derivation of a scalar leading edge equation is not as straightforward, and also leaves some freedom, as we discuss below and for an example in Appendix L. Nevertheless, we will see that the results summarized in Table 2 are robust, in that they do not depend on the particular choice made. We discuss examples of single PDEs in Sections 5.6.1 and 5.6.4, and an example of sets of PDEs in 5.6.2. Of course, if one has a PDE for a single scalar field $\phi$, one can directly take the Fourier-Laplace transform without writing $\phi$ as a vector field. This yields a slight generalization of (5.73), the most important difference being that $H$ then also depends on $\omega$. Our results can obviously also be obtained via this route (see Section 5.5.2 for further details).

Difference-differential equations. When we have difference equations in space, the equations can also be reduced to the above form - the only difference is that upon Fourier transformation in space, the $k$-values can be restricted to lie in a finite interval (the "Brillouin zone", in physics terminology). An example will be discussed in Section 5.6.3. Likewise, when we analyze a dynamical equation with finite time difference, the Laplace integral can be replaced by a sum over integer times but the "frequency" remains a continuous variable. The only difference is that upon Laplace inversion, the integral is over a finite interval of $\omega$ values. Examples of difference equations in both space and time, arising from numerical schemes, can be found in Section 5.6.6.

Equations with memory or spatial kernels. If the equation has memory and/or spatial kernels of the type $\int \mathrm{d} x^{\prime} \int_{0}^{t} \mathrm{~d} t^{\prime} K\left(x-x^{\prime}, t-t^{\prime}\right) \phi\left(x^{\prime}, t^{\prime}\right)[103,104]$, then upon Fourier-Laplace transformation these just give rise to terms of the form $\hat{K}(k, \omega) \hat{\phi}(k, \omega)$ in (5.73), as will be illustrated with a simple example in Section 5.6.5. The only difference with the case of PDEs from this point of view then is that the elements $\hat{S}_{m n}$ are not polynomials in $\omega$ and $k$, but more general functions of these arguments.

\subsubsection{Long-time asymptotics of the Green's function via a Fourier-Laplace transformation}

We now return to the problem of extracting the long-time behavior of the dynamical equation (5.73) in LaplaceFourier representation. In analogy with our earlier analysis of PDEs, and following [56,58], we introduce the Green's function $^{23} \underline{\underline{G}}(k, \omega)$ of the linear equations, defined by

$$
\underline{\underline{\hat{G}}}(k, \omega)=\underline{\underline{\hat{S}}}(k, \omega)^{-1} .
$$

$\underline{\underline{S}}^{-1}$ is the inverse of the matrix $\underline{\underline{\hat{S}}}$. Eq. (5.73) now immediately can be solved as

$$
\underline{\hat{\phi}}(k, \omega)=\underline{\underline{\hat{G}}}(k, \omega) \cdot \underline{\underline{\tilde{H}}}(k) \cdot \underline{\tilde{\phi}}(k, t=0) .
$$

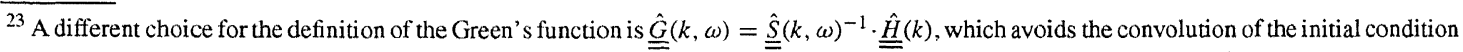
with $\underline{\underline{H}}(z)$ in (5.79), and also for equations of the form (5.44) leads to the easier expression $\underline{\underline{\hat{G}}}(k, \omega)=(\underline{\underline{\tilde{T}}}(k)-\mathrm{i} \omega \underline{\underline{\tilde{I}}})^{-1}$. The advantage of the choice (5.74) is that we consistently work with derivatives of $S=\operatorname{det} \underline{\underline{\hat{S}}}$.
} 
We write the eigenvectors and eigenvalues of $\underline{\underline{\hat{S}}}$ in analogy to (5.50)-(5.52) as

$$
\underline{\underline{S}}(k, \omega) \cdot \underline{\hat{U}}_{m}(k, \omega)=u_{m}(k, \omega) \underline{\hat{U}}_{m}(k, \omega) .
$$

The determinant of $\hat{S}$ can now be written as

$$
S(k, \omega)=\operatorname{det} \underline{\underline{\hat{S}}}(k, \omega)=\prod_{m=1}^{M} u_{m}(k, \omega),
$$

and the characteristic equation

$$
u_{m}\left(k, \omega_{m}(k)\right)=0
$$

determines the dispersion relation $\omega_{m}(k)$ of the mode with eigendirection $\underline{\hat{U}}_{m}(k, \omega)$. Note that each eigenvalue $u_{m}(k, \omega)$ may be a nonlinear function of $k$ and $\omega$. Therefore it can happen that the equation $u_{m}(k, \omega)=0$ specifies more than one branch $\omega(k)$ of the dispersion relation. For simplicity, we will not distinguish this possibility with our notation, but we stress that our results are generally valid. For equations of the form (5.46), we can identify $u_{m}(k, \omega)=A_{M}(k)\left(i \omega_{m}(k)-\mathrm{i} \omega\right)$ and $\underline{\hat{U}}_{m}(k, \omega)=\underline{\tilde{U}}_{m}(k)$.

Upon inverting the Fourier and Laplace transformations, where the Laplace inversion requires a sufficiently large real $\gamma$, we now find for the Green's function in the comoving frame $\xi=x-v t$ :

$$
\begin{aligned}
& \underline{\phi}(\xi, t)=\int \mathrm{d} y \underline{\underline{G}}(\xi-y, t) \cdot \int \mathrm{d} y^{\prime} \underline{\underline{H}}\left(y-y^{\prime}\right) \cdot \underline{\phi}\left(y^{\prime}, 0\right), \\
& \underline{\underline{G}}(\xi, t)=\int_{-\mathrm{i} \gamma-\infty}^{-\mathrm{i} \gamma+\infty} \frac{\mathrm{d} \omega}{2 \pi} \int_{-\infty}^{\infty} \frac{\mathrm{d} k}{2 \pi} \exp \{\mathrm{i} k \xi-\mathrm{i}(\omega-v k) t\} \underline{\underline{\hat{G}}}(k, \omega), \\
& \underline{\underline{\hat{G}}}(k, \omega)=\sum_{m=1}^{M} \frac{\hat{\underline{U}}_{m}(k, \omega) \times \underline{\hat{U}}_{m}^{\dagger}(k, \omega)}{u_{m}(k, \omega)} .
\end{aligned}
$$

The expression for $\underline{\underline{G}}(\xi, t)$ is the immediate generalization of (5.54). When we evaluate the Fourier-Laplace inversion of $\underline{G}(\xi, t)$ in the long-time limit, each term in the sum (5.79) can be evaluated by the so-called "pinch point" analysis [56,58] making use of expansions about zeroes of $u_{m}(k, \omega)$. We then need to deform not only the contour of $k$-integration, as in the saddle point analysis in the previous sections, but also the contour of $\omega$ integration. The pinch point analysis is based on first evaluating the $k$-integral, and then the resulting $\omega$-integral. Alternatively, we can extract the long-time dynamics by first closing the $\omega$-contour, and then performing the $k$-integral. This last route is closer to that of Section 5.4. For a further discussion of both approaches and of the global conditions that determine which of the saddle points or pinch points is dynamically relevant, we refer to Appendix M. As before, we use $*$ to denote the appropriate solution that satisfies these conditions.

As always, there can in principle be several saddle point or pinch point solutions through which the integration contour can be deformed, and if this happens, the relevant one is the one corresponding to the largest velocity $v^{*}$. If we again write $u_{1}(k, \omega)$ for the eigenvalue on which this solution lies and as before use a superscript * for functions which are written in terms of the transformed variable $\Omega$ and $q$ as in (5.59) and (5.60), the saddle or pinch point equations assume their familiar form

$$
\begin{aligned}
& u_{1}\left(k^{*}, \omega^{*}\right)=0 \Leftrightarrow u_{1}^{*}(0,0)=0, \\
& \left.\left(\partial_{k}+v^{*} \partial_{\omega}\right) u_{1}(k, \omega)\right|_{k^{*}, \omega^{*}}=\left.0 \quad \Leftrightarrow \quad \partial_{q} u_{1}^{*}(q, \Omega)\right|_{0,0}=0 .
\end{aligned}
$$


Note that since $S$ is the product of all eigenvalues, cf. Eq. (5.77), these equations are equivalent to those given before in terms of $S$, Eq. (5.62). Likewise, we get for the long-time asymptotics of the Green's function the immediate generalization of (5.55),

$$
\underline{\underline{G}}(\xi, t)=\mathrm{e}^{-\lambda^{*} \xi} \frac{\exp \left\{-\xi^{2} /(4 D t)\right\}}{(4 \pi D t)^{1 / 2}} \frac{\tilde{U}_{1}\left(k^{*}, \omega^{*}\right) \times \underline{\tilde{U}}_{1}^{\dagger}\left(k^{*}, \omega^{*}\right)}{\left.\mathrm{i} \partial_{\omega} u_{1}(k, \omega)\right|_{\left(k^{*}, \Omega^{*}\right)}}+\cdots,
$$

which is our usual Gaussian expression again, with $D$ given by its familiar expression (5.68).

Our strategy in deriving the long-time front dynamics is always to use the long-time evaluation of the Green's function just to show how the pulled velocity $v^{*}$ and the dominant exponential behavior $\mathrm{e}^{-\lambda^{*} \xi}$ emerge, and to motivate why the leading edge variables $\psi(\xi, t)$ have essentially slow diffusive dynamics. The analysis of the slow $\psi$ dynamics and the matching to the front interior is most properly done by going back to the PDE(s) for the spatio-temporal evolution of $\psi$. Switching back to the space-time formulation for $\psi$ comes out most directly from Fourier-Laplace inversion of the small- $q$ and small- $\Omega$ expansion of the $\psi$-equation. Indeed, for $\psi(\xi, t)$ the appropriate Green's function is $\mathrm{e}^{\lambda^{*} \xi} \underline{\underline{G}}(\xi, t)$ and according to (5.79), we have

$$
\mathrm{e}^{\lambda^{*} \xi} \underline{\underline{G}}(\xi, t)=\int \frac{\mathrm{d} \Omega}{2 \pi} \int \frac{\mathrm{d} q}{2 \pi} \exp \{\mathrm{i} q \xi-\mathrm{i} \Omega t\} \underline{\underline{\hat{G}}}^{*}(q, \Omega),
$$

which confirms that $\Omega$ and $q$ are the proper Fourier-Laplace variables of the leading edge variable $\psi$.

\subsubsection{The case of a single field}

In contrast to our earlier matrix notation, a single equation for a single field $\phi(x, t)$ after Fourier-Laplace transformation can also be written in a scalar form:

$$
S(k, \omega) \hat{\phi}(k, \omega)=\text { function }\left\{k, \tilde{\phi}(k, 0),\left.\partial_{t} \tilde{\phi}(k, t)\right|_{t=0}, \ldots\right\} .
$$

The most common and direct way to arrive at the above equation is by performing a Fourier-Laplace transformation on the original dynamical equation. In this case one immediately gets the characteristic function $S(k, \omega)$ on the LHS, while the partial integrations (or partial summations in the case of difference equations, where also the derivatives in the initial condition terms are replaced by finite difference versions) of higher order temporal derivatives yield $\omega$-dependent initial condition terms on the RHS in (5.83). Of course, we can also arrive at this equation via the route of Section 5.4, where we introduced a vector notation for a scalar PDE of higher order in time, so that the dynamical equation is of the matrix form (5.73). Indeed, when we calculate det $\underline{\underline{S}}(k, \omega)$ with $\underline{\underline{S}}(k, \omega)=A_{M}(k)(\underline{\underline{\hat{T}}}(k)-i \omega \underline{\underline{1}})$ by developing the determinant along the last row of the matrix, one easily sees that one just retrieves the above result.

Of course, the asymptotic analysis of $\phi(\xi, t)$ parallels the earlier discussion of Section 5.4, irrespective of whether or not the equation is written in vector form. Again, the asymptotic spreading speed is given by a saddle point of $S(k, \omega)$. However, as we have seen, for analyzing the proper front dynamics we want to return to the dynamical equation for the leading edge variable $\psi$. For the case of a PDE, this can be done simply by transforming the original equation for $\phi$ to the leading edge representation $\psi(\xi, t)=\mathrm{e}^{\lambda^{*} \xi} \phi(\xi, t)$, but for difference equations or equations with memory terms, additional steps are clearly necessary. The general analysis is based on the observation that in the leading edge representation, the dynamical equation is of the form

$$
S^{*}(q, \Omega) \hat{\psi}(q, \Omega)=\text { initial condition terms. }
$$

If we expand $S^{*}$ in $q$ and $\Omega_{m}$ and perform an inverse Fourier-Laplace transform, we immediately arrive at the $\operatorname{PDE}(5.57)$ for $\psi(\xi, t)$ with coefficients $b_{m n}$ given in terms of the derivatives of $S^{*}$ according to (5.61)! From there 
on, the analysis completely follows the one in the last part of Section 5.4, and we recover again all our familiar expressions for the relaxation of the front velocity and the profile.

We stress that for a given equation, the transformation to the leading edge variable can be done exactly. If this is done for a PDE, we again get a PDE of finite order. As no approximations are made, the resulting equation still allows one to study the fast or small-scale dynamics in the linear region as well. For finite difference equations or for integro-differential equations, the transformation to the leading edge variable $\psi$ still results in a finite difference equation or an integro-differential equation: the usual PDE for $\psi$ then only emerges if in addition a gradient expansion is made for $\psi$. Such an expansion will obviously contain an infinite number of terms. (We will see explicit examples of this in Sections 5.6.3, 5.6.5 and 5.6.6.) Normally, such an expansion is not of much use. However, when we turn to the long-time relaxation towards pulled fronts in the leading edge, $\psi$ becomes arbitrarily smooth and slow and hence the derivatives become nicely ordered. Moreover, the long-time large-scale relaxation of $\psi$ corresponds precisely to the low-frequency small-wave number behavior of the Fourier-Laplace transform and that is why the expansion of $S^{*}$ gives the proper evolution equation to analyze the front relaxation: as (5.61) shows, the coefficients $b_{m n}$ in this equation are then nothing but the expansion coefficients of the characteristic equation $S^{*}(q, \Omega)$ for small $q$ and $\Omega$. In other words, independently of whether we started from a differential, a difference or an integro-differential equation, we find at this point always the same PDE for the leading edge variable $\psi$, and hence the same expression for the velocity relaxation!

Let us finally remark, that instead of the leading edge transformation, we could also have performed a leading edge projection onto the slow dynamics, as discussed in the following section. We will show, that the results of Table 2 do not depend on this choice.

\subsubsection{The case of a set of fields and possible projections}

For dynamical equations which inherently consist of sets of equations for more than one field, one obviously can only arrive at an equation for a scalar variable $\psi$ by some kind of projection onto the slow direction. The way in which one projects out the slow dynamics clearly entails a certain freedom of choice. For a given equation the "best" choice may be obvious, but in general there is some ambiguity. We illustrate this explicitly in Appendix L.

We note first, that a vector field $\underline{\hat{\psi}}(q, \Omega)$ can be decomposed into its dynamical components $\hat{\pi}_{m}(q, \Omega)$ as

$$
\begin{aligned}
& \underline{\hat{\psi}}(q, \Omega)=\sum_{m=1}^{M} \hat{\pi}_{m}(q, \Omega) \underline{\hat{U}}_{m}^{*}(q, \Omega), \\
& \hat{\pi}_{m}(q, \Omega)=\underline{\hat{U}}_{m}^{* \dagger}(q, \Omega) \cdot \underline{\hat{\psi}}(q, \Omega),
\end{aligned}
$$

where the superscript ${ }^{*}$ on the eigenvectors $\underline{U}_{m}$ and eigenvalues $u_{m}$ is to remind us that these are written in terms of the variables $q$ and $\Omega$.

Each $\hat{\pi}_{m}(q, \Omega)$ has its own dynamics, cf. (5.84),

$$
u_{m}^{*}(q, \Omega) \hat{\pi}_{m}(q, \Omega)=\text { initial condition terms } m .
$$

The natural projection onto a scalar leading edge variable is thus onto the eigendirection with the largest $v^{*}$, which we denote with $\hat{U}_{1}^{*}(q, \Omega)$. We then identify the scalar leading edge variable with $\hat{\pi}_{1}(q, \Omega)$. Inverting now the Fourier-Laplace transformation, we find a PDE for $\pi_{1}(\xi, t)$ of the form (5.57) with the coefficients

$$
b_{m n}^{(1)}=\left.\frac{\left(\mathrm{i} \partial_{\Omega}\right)^{m}}{m !} \frac{\left(-\mathrm{i} \partial_{q}\right)^{n}}{n !} u_{1}^{*}(q, \Omega)\right|_{(q=\Omega=0)} .
$$


Table 5

The saddle or pinch point equations, determining $v^{*}, k^{*}=\mathrm{i} \lambda^{*}$ and $D$ for a given characteristic function $S(k, \omega)=\operatorname{det} \underline{\underline{S}}(k, \omega)$. If there are several dynamically relevant saddle point solutions (for relevance see Appendix $\mathrm{M}$ ), take the one with the largest $v^{*}$

$$
\begin{aligned}
& \text { Definition of } \omega_{m}(k) \text { : } \\
& \left.S\left(k, \omega_{m}(k)\right)=0 \quad \text { (def.: } \lambda^{*}=i k^{*}\right) \\
& \text { Saddle point eqs.: } \\
& S\left(k^{*}, \omega^{*}\right)=0 \quad \Longleftrightarrow \quad \omega^{*}=\omega_{m}\left(k^{*}\right) \\
& \left.\left(\partial_{k}+v^{*} \partial_{\omega}\right) S\right|_{\left(k^{*}, \omega^{*}\right)}=0 \Longleftrightarrow v^{*}=\left.\frac{\partial \omega_{m}(k)}{\partial k}\right|_{\left(k^{*}, \omega^{*}\right)} \\
& \operatorname{Im}\left(\omega^{*}-v^{*} k^{*}\right)=0 \Longleftrightarrow v^{*}=\frac{\operatorname{Im} \omega_{m}\left(k^{*}\right)}{\operatorname{Im} k^{*}} \\
& D=\left.\frac{-i\left(\partial_{k}+v^{*} \partial_{\omega}\right)^{2} S}{2 \partial_{\omega} S}\right|_{\left(k^{*}, \omega^{*}\right)} \Longleftrightarrow D=\left.\frac{i \partial^{2} \omega_{m}(k)}{2 \partial k^{2}}\right|_{\left(k^{*}, \omega^{*}\right)}
\end{aligned}
$$

In general, only saddle points with $\operatorname{Re} D>0$ are relevant. In this paper only saddle points with real $D$ are considered.

Defining the saddle point parameters just as in (5.62) and (5.63) for Eq. (5.57), they in general will depend on whether we derived the coefficients from $S$ or from $u_{1}$. However, we will argue below that the saddle point parameters $v^{*}$, $\lambda^{*}$ and $D$ do not depend on this choice.

Though the projection onto $\hat{U}_{1}^{*}(q, \Omega)$ is formally the simplest one, the direction of projection is actually not very practical, as it depends on $q$ and $\Omega$. In practice, one will want to project along a fixed direction. Our previous analysis, summarized by Eq. (5.81), indeed suggested to project the long-time dynamics of the Green's function onto $\underline{U}_{1}\left(k^{*}, \omega^{*}\right)=\underline{\hat{U}}_{1}^{*}(0,0)$. Projection of $\underline{\hat{\psi}}(q, \Omega)$ onto this eigendirection yields

$$
\hat{\psi}^{\mathrm{p}}(q, \Omega)=\underline{\hat{U}}_{1}^{*}(0,0) \cdot \underline{\hat{\psi}}(q, \Omega)=\sum_{m=1}^{M} \hat{\pi}_{m}(q, \Omega) \underline{\hat{U}}_{1}^{*^{\dagger}}(0,0) \cdot \underline{\hat{U}}_{m}^{*}(q, \Omega) .
$$

Now only for $q \approx 0 \approx \Omega$, we have $\hat{\psi}^{\mathrm{p}}(q, \Omega) \approx \hat{\pi}_{1}(q, \Omega)$, while for finite $q$ and $\Omega$, also $\hat{\pi}_{m}(q, \Omega)$ with $m>1$ will contribute. Inverting the Fourier-Laplace transform and working in the frame $\xi=x-v^{*} t$, we find the contributions from $\hat{\pi}_{m>1}$ to decay exponentially in time. Such contributions we encountered already a number of times before, for the first time in Section 2.5. The more important contribution comes from the coefficient of $\hat{\pi}_{1}$, which is $\hat{\hat{U}}_{1}^{* \dagger}(0,0) \cdot \hat{U}_{1}^{*}(q, \Omega)=1-\mathrm{O}(q, \Omega)$. These algebraic corrections in $q$ and $\Omega$ actually modify $b_{m n}$ for the projection $\hat{\psi}^{\mathrm{p}}(q, \Omega)$ in comparison with (5.88), except for the diffusion coefficient $D$, as we will see below.

Still, other projections might be physically useful as illustrated in the explicit example of Appendix L. We now turn to the consequences of all these different choices.

\subsubsection{The freedom of projection and the universality of Tables 2 and 5}

At first sight, the leading edge transformation or the different leading edge projections each determine their own saddle or pinch point equations or expansion parameters $b_{m n}$; compare, e.g., (5.61) with (5.88).

Nevertheless, the definition of the saddle or pinch point parameters $v^{*}, \lambda^{*}$ and $D$ in Table 5 does not depend on the choice of the leading edge transformation or projection, and hence the universal relaxation results for the velocity $v(t)$ and the shape $\Phi_{v(t)}$ in Table 2 are independent of these as well.

For the saddle/pinch point equations of Table 5 this conclusion is based on two observations: (i) $S(k, \omega)$ contains $u_{1}(k, \omega)$ as a factor (5.77). The saddle point is determined by a double root in $k$ of $u_{1}(k, \omega)$, which can be 
written as

$$
u_{1}^{*}(q, \Omega)=b_{10}^{(1)}\left(-\mathrm{i} \Omega+D q^{2}+\cdots\right)=u_{1}(k, \omega)=b_{10}^{(1)}\left(-\mathrm{i}\left(\omega-v^{*} k\right)+D\left(k-k^{*}\right)^{2}+\cdots\right) .
$$

$D$ here obviously is defined as $D=-b_{02}^{(1)} / b_{10}^{(1)}$ with $b_{m n}^{(1)}$ from (5.88). The root (5.90) fully determines the lowest derivatives of $S=\prod_{m} u_{m}$ at the saddle point $q=0=\Omega-$ up to a constant prefactor, resulting from the other factors in $S$. (ii) The saddle point parameters are defined by homogeneous equations (5.62) or ratios of derivatives (5.63). So the prefactors depending on differentiation of either $u_{1}$ or $S$ will cancel in the equations that determine $v^{*}, \lambda^{*}$ and $D$. In particular, $D$ defined by $D=-b_{02} / b_{10}$ in (5.63) is identical with $D=-b_{02}^{(1)} / b_{10}^{(1)}$ here and with other $D$ 's resulting from different projections.

The subleading terms $D_{3}$ and $w$ for the scalar leading edge variable in (5.64), in contrast, do depend on the choice of projection. Hence, as there always will be a leading edge equation of the form (5.64), and as the universal results summarized in Table 2 do not depend on the values of $D_{3}$ or $w$, Table 2 is a universal result, independent of the particular projection chosen. The subleading contribution $g_{0}(z)$ in the leading edge will always be solved as in (5.69), so it will not depend on initial conditions, but will depend on the direction of projection through the parameters $D_{3}$ and $w$.

In conclusion, we reiterate that the relaxation results also apply to dynamical equations other than PDEs, because the dynamics of the leading edge representation $\psi$ becomes arbitrarily slow and diffusive for long times. This allows one to do a gradient expansion in time and space for $\psi$, even if the original equations are not PDEs! In this case the path of analysis via the Fourier-Laplace transformation and pinch point analysis is necessary. For equations that are of differential form in time, Fourier transformation in space and saddle point analysis is sufficient.

\subsection{Applications}

In this section, we support the above arguments by summarizing the results of numerical simulations of three equations - a spatially fourth order PDE, a set of two coupled PDEs and a difference-differential equation which are all in complete agreement with our predicted universal relaxation trajectory as in Table 2, consisting of the velocity convergence (5.70), the slaved interior (5.71), and the crossover to a diffusive type of dynamics in the leading edge for $\xi \gtrsim \sqrt{t}$. We also briefly consider a PDE with second order temporal derivatives, an extension of the nonlinear diffusion equation with a memory kernel, and the discretization corrections in the Euler and in the semi-implicit numerical integration method for a nonlinear diffusion equation. The last results were used already in Section 4 in our numerical study of the nonlinear diffusion equation.

\subsubsection{The EFK equation}

The EFK ("extended Fisher Kolmogoroff") equation is an extension of the nonlinear diffusion equation $[64,65]$, which has been investigated quite intensely in the mathematical literature [101]. It reads

$$
\partial_{t} \phi=\partial_{x}^{2} \phi-\gamma \partial_{x}^{4} \phi+\phi-\phi^{3}
$$

A straightforward calculation [65] shows that the saddle point equations (5.13), (5.16) and (5.17) yield

$$
v^{*}=2 \lambda^{*}\left(1-2 \gamma \lambda^{*^{2}}\right), \quad \lambda^{*}=\left(\frac{1-(1-12 \gamma)^{1 / 2}}{6 \gamma}\right)^{1 / 2}, \quad D=(1-12 \gamma)^{1 / 2} \text { for } \gamma<\frac{1}{12} .
$$

For $\gamma>\frac{1}{12}$, the saddle point solution has $\operatorname{Re} k^{*} \neq 0$, and in agreement with this, the pulled fronts in this equation are then found to be non-uniformly translating and to generate periodic patterns [64]. We will therefore focus here on the regime $\gamma<\frac{1}{12}$. The arguments of the Appendix of [65] for the multiplicity of front solutions (summarized 


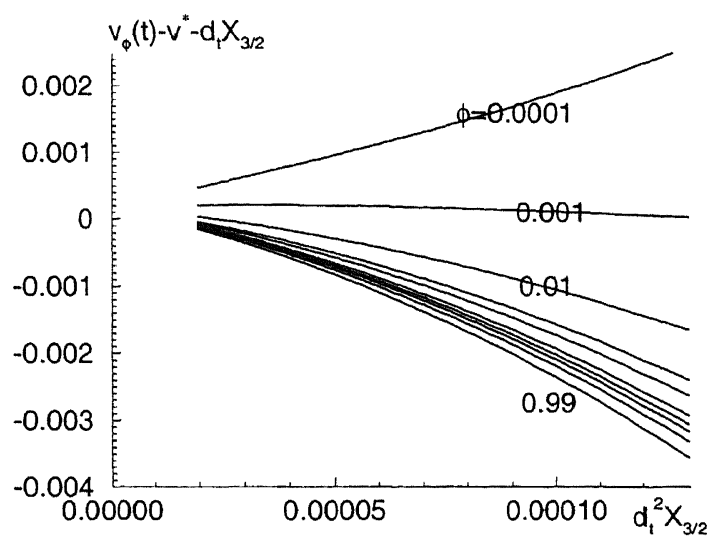

Fig. 11. Velocity relaxation in the EFK equation (5.91) for $\gamma=0.08$ : plot of $v_{\phi}(t)-v^{*}-\dot{X}_{3 / 2}$ as a function of $\ddot{X}_{3 / 2}$ as in Fig. 8(a) for times $60 \leq t \leq 200$. System size $L=200$, front position $x_{0}=25$, initial steepness $\lambda_{\text {init }}=20$ in (4.2). Grid sizes $\Delta x=0.01=\Delta t$.

in Appendix $\mathrm{H}$ ) give evidence that this equation indeed admits a family of uniformly translating fronts in this regime. One also can prove that the front cannot propagate with a velocity larger than $v^{*}$ if the initial conditions are sufficiently steep [72,99]. The convergence towards the pulled front solution should therefore be given by Eq. (5.40) for $v(t)$ and Eq. (5.31) or Eqs. (5.38) and (5.39) for the interior of the leading edge of the front profile. Fig. 11 shows some of the results of our numerical simulations for $v(t)$ at $\gamma=0.08$. This value of $\gamma$ is just below the bifurcation value $\gamma_{c}=\frac{1}{12}=0.083$. The plot is of the same type as in Fig. 8(a) for the nonlinear diffusion equation.

The numerical grid sizes of the simulation are $\Delta x=0.01=\Delta t$. The system size is $L=200$, the initial condition is characterized by $\lambda_{\text {init }}=20$ and $x_{0}=25$. The analytical prediction for $\gamma=0.08$ is, according to (5.92), in the limit $\Delta x \rightarrow 0, \Delta t \rightarrow 0: D=0.2, \lambda^{*}=\left(\frac{5}{3}\right)^{1 / 2}=1.29$, and $v^{*}=\frac{4.4}{3} \lambda^{*}=1.89$. The ratio between the $1 / t$ - and the $1 / t^{3 / 2}$-contribution in $v(t)$ according to (5.40) is measured on the timescale

$$
T=\frac{1}{\lambda^{*^{2}} D}
$$

as in the dimensional analysis (5.36). For $\gamma=0.08$, we have $T=3$. The plot of Fig. 8(a) gave good results from time $t=20$ on, where $T=1$. It is therefore consistent that the plot of Fig. 11 with $T=3$ is good from times $t=60 \mathrm{on}$. We thus plot here the time interval $60 \leq t \leq 200$. One can already anticipate from the plot that again a correction of $v^{*}$ for the numerical finite difference code will be required if we proceed to even higher precision. In conclusion, we find the results to be in full accord with our analytical predictions.

\subsubsection{The streamer equations}

Streamers are discharge patterns which result from the competition between an electron avalanche formation due to impact ionization, and the screening of the electric field by space charges in the ionized region. For planar streamer fronts, the equation for the electron density $\sigma$ and electric field $E$ are [15]

$$
\partial_{t} \sigma=D_{\sigma} \partial_{x}^{2} \sigma+\partial_{x}(\sigma E)+\sigma f_{\mathrm{str}}(E), \quad \partial_{t} E=-D_{\sigma} \partial_{x} \sigma-\sigma E,
$$

where we have assumed that in the region $x \gg 1$, where the electron density vanishes $\sigma^{+}=\sigma(x \rightarrow \infty, t)=0$, the electric field $E^{+}=E(x \rightarrow \infty, t)$ does not change in time: $\partial_{t} E^{+}=0$. The field-dependent ionization rate has a functional form like $f_{\text {str }}(E)=|E| \exp \{-1 /|E|\}$. This is the functional form we use in our simulations. The state $(\sigma, E)=\left(0, E^{+}\right)$is unstable, and also for these equations, it is known [15] that they admit a one parameter 


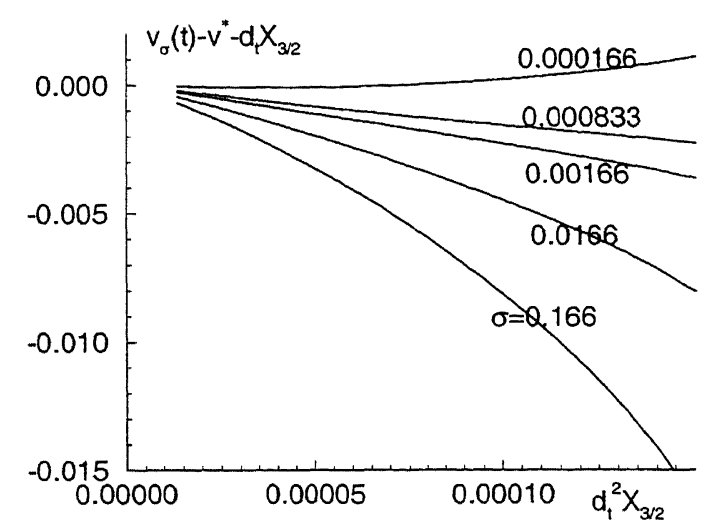

Fig. 12. Velocity relaxation in the streamer equations (5.94) for $E^{+}=-1$ and $D=0.1$, plotted as in Figs. 8 (a) and 11 for times $40 \leq t \leq 200$ Initial condition: Gaussian electron density $\sigma(x, 0)=0.9 \exp \left\{-x^{2}\right\}$ (thus $\lambda_{\text {init }}=\infty$ ), $E(x, 0)=-1$. System size $L=400$, front position shifted back to $x_{0}=100$, after it is reached. Grid sizes: $\Delta x=0.01, \Delta t=0.0025$.

family of uniformly translating front solutions. The dispersion relation for linear perturbations about the unstable state $\sigma=0, E=E^{+}<0$ reads $-\mathrm{i} \omega(k)=\mathrm{i} k E^{+}+f_{\text {str }}\left(E^{+}\right)-D_{\sigma} k^{2}$, where we choose to analyze the leading edge in a projection onto the $\sigma$-axis. The saddle point equations (5.13), (5.16) and (5.17) then yield

$$
\begin{aligned}
& v^{*}=-E^{+}+2\left(D_{\sigma} f_{\mathrm{str}}\left(E^{+}\right)\right)^{1 / 2}, \quad \lambda^{*}=\left(\frac{f_{\mathrm{str}}\left(E^{+}\right)}{D_{\sigma}}\right)^{1 / 2}, \\
& D=D_{\sigma} .
\end{aligned}
$$

Again, the simulations of these equations show that the velocity convergence follows our analytical prediction (5.40). An example of our results is shown in Fig. 12 in a plot as in Figs. 8(a) and 11, where we track various levels of the electron density $\sigma$. The dimensionless time is $T=1 / f_{\text {str }}\left(E^{+}\right)=\mathrm{e}^{1}=2.718$ for $E^{+}=-1$. We plot our data for times $40 \leq t \leq 200$, and again find our predictions to hold.

\subsubsection{A difference-differential equation}

We now summarize some key elements of our analysis [102] of the difference-differential equation,

$$
\partial_{t} C_{j}(t)=-C_{j}+C_{j-1}^{2}, \quad C_{0}(t)=0, \quad C_{j \gg 1}(t)=1,
$$

with $j$ integer. This equation originates from kinetic theory [17]. If we transform with $\phi_{j}(t)=1-C_{j}(t)$ to

$$
\partial_{t} \phi_{j}(t)=-\phi_{j}+2 \phi_{j-1}-\phi_{j-1}^{2}, \quad \phi_{0}(t)=1, \quad \phi_{j \gg 1}(t)=0,
$$

we have our usual notation with the state $\phi_{j}=0$ being unstable and the state $\phi_{j}=1$ stable. As usual, we consider fronts between these states starting from sufficiently steep initial conditions. It is easy to see that such initial conditions will create a pulled front [102].

Eq. (5.98) provides the first illustration of our argument from Section 5.5 that our analysis applies to difference equations as well - with the only difference that the spatial Fourier modes $k$ now extend over a finite interval or "Brillouin zone" $0 \leq k<2 \pi$ only. Substitution of the Fourier ansatz $\phi_{j} \sim \exp \{-\mathrm{i} \omega t+\mathrm{i} k j\}$ into the equation of motion linearized about the unstable state $\phi_{j}=0$,

$$
\partial_{t} \phi_{j}=-\phi_{j}+2 \phi_{j-1},
$$


yields the dispersion relation

$$
-\mathrm{i} \omega(k)=2 \mathrm{e}^{-\mathrm{i} k}-1 \Leftrightarrow s(\lambda)=2 \mathrm{e}^{\lambda}-1
$$

As discussed before, the long-time asymptote of the leading edge is again determined by the saddle point which obeys (5.13), (5.16) and (5.17). This results in

$$
v^{*}=2 \mathrm{e}^{\lambda^{*}}=\frac{2 \mathrm{e}^{\lambda^{*}}-1}{\lambda^{*}}
$$

When we choose the solution with $v^{*}>0$, the saddle point equations are solved by

$$
\begin{aligned}
-\mathrm{i} k^{*} & =\lambda^{*}>0 \text { real }, & v^{*}=2 \mathrm{e}^{\lambda^{*}}=4.31107, & \lambda^{*}=\frac{2 \mathrm{e}^{\lambda^{*}}-1}{2 \mathrm{e}^{\lambda^{*}}}=0.768039, \\
D & =D_{2}=\frac{1}{2} v^{*}, & D_{n} & =\frac{(-)^{n} v^{*}}{n !} .
\end{aligned}
$$

The $D_{n}$ values are determined from (5.28). We now perform the leading edge transformation

$$
\phi_{j}(t)=\mathrm{e}^{-\lambda^{*} \xi} \psi(\xi, t), \quad \xi=j-v^{*} t .
$$

The large-time, small-gradient expansion in the leading edge now results in the PDE

$$
\partial_{t} \psi=D \partial_{\xi}^{2} \psi+D_{3} \partial_{\xi}^{3} \psi+\cdots
$$

The velocity convergence is again given by (5.40), with $v^{*}, \lambda^{*}$ and $D$ given by (5.102). We do find indeed that the fronts in this equation are pulled, and that the velocity convergence follows (5.40). This is illustrated in Fig. 13, where we plot $\left(v(t)-v^{*}+3 /\left(2 \lambda^{*} t\right)\right) / t^{-3 / 2}$ as a function of $1 / \sqrt{t} . v(t)=\dot{x}(t)$ is the velocity of the front defined as $x(t)=\sum_{j=0}^{\infty} \phi_{j}(t)$. The curve in Fig. 13 should extrapolate to $3 /\left(2 \lambda^{*}\right)\left(\pi /\left(\lambda^{*^{2}} D\right)\right)^{1 / 2}=3.0699$ as $1 / \sqrt{t} \rightarrow 0$. This predicted asymptote is marked by the cross on the axis. Indeed, the data of $\left(v(t)-v^{*}+3 /\left(2 \lambda^{*} t\right)\right) / t^{-3 / 2}$ for $40 \leq t \leq 4000$ extrapolate very well to the predicted asymptote, especially in view of the fact, that $t^{3 / 2} \approx 2 \times 10^{5}$ at the latest times. The slight offset at the end might be due either to finite system size $L$ or to finite numerical discretization $\Delta t$.

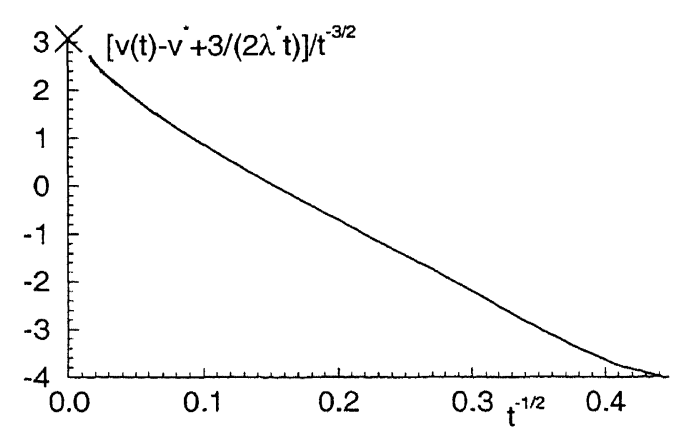

Fig. 13. Velocity relaxation for the difference-differential equation (5.97), where $v(t)=\dot{x}(t)$, and $x(t)=\sum_{j=0}^{\infty} \phi_{j}(t)$, see Eq. (5.98). Plot of $\left(v(t)-v^{*}+3 /\left(2 \lambda^{*} t\right)\right) / t^{-3 / 2}$ as a function of $1 / \sqrt{t}$ for times $40 \leq t \leq 4000$. The curve is predicted to extrapolate to $c_{3 / 2}$ as $1 / \sqrt{t} \rightarrow 0$. The predicted value of $c_{3 / 2}$ is marked by the cross on the axis. Initial condition $\phi_{j}(0)=\exp \left\{-j^{2}\right\}$. System size $N=4000$ grid points. Front shifted back to $n_{0}=75$, after it has been reached. Temporal grid size $\Delta t=0.0005$. 
5.6.4. Diffusion equation with second order time derivative

Quite recently, it was shown [77] that, not surprisingly, fronts in a second order extension of the F-KPP equation,

$$
\tau_{2} \frac{\partial^{2} \phi}{\partial t^{2}}+\frac{\partial \phi}{\partial t}=\frac{\partial^{2} \phi}{\partial x^{2}}+\phi-\phi^{3}
$$

are also pulled. One interesting aspect of this equation is that while the diffusive spreading in a first order diffusion equation is, in a sense, infinitely fast, the second order term gives a finite speed of propagation of the disturbances.

As discussed in Section 5.4, our results immediately apply to this equation, so the velocity and front relaxation is then given by Eqs. (5.70) and (5.71), with

$$
v^{*}=\frac{2}{\left(1+4 \tau_{2}\right)^{1 / 2}}, \quad \lambda^{*}=\left(1+4 \tau_{2}\right)^{1 / 2}, \quad D=\frac{1}{\left(1+4 \tau_{2}\right)^{2}} .
$$

The expression for $D$ nicely illustrates the effective renormalization of the diffusion coefficient due to the second order time derivative.

5.6.5. An extension of the F-KPP equation with a memory kernel

As an example of an equation with a memory kernel, consider the extension of the F-KPP equation

$$
\partial_{t} \phi(x, t)=\partial_{x}^{2} \phi(x, t)+\int_{0}^{t} \mathrm{~d} t^{\prime} K\left(t-t^{\prime}\right) \phi\left(x, t^{\prime}\right)-\phi^{k}(x, t) \quad(k>1) .
$$

Upon Fourier-Laplace transformation as in (5.18), this equation is a scalar version of (5.73) with $S(k, \omega)=$ $i \omega-k^{2}+\tilde{K}(\omega)$, and so according to our discussion of Section 5.5 , our analysis directly applies. If we take for instance

$$
K\left(t-t^{\prime}\right)=\frac{1}{\pi^{1 / 2} \tau_{3}} \exp \left\{\frac{-\left(t-t^{\prime}\right)^{2}}{4 \tau_{3}^{2}}\right\},
$$

the equation reduces to the F-KPP equation in the limit $\tau_{3} \rightarrow 0$, and the characteristic equation becomes

$$
\lambda^{2}-s+\exp \left\{\tau_{3}^{2} s^{2}\right\} \operatorname{erfc}\left(\tau_{3} s\right)=0,
$$

where we follow the notation of Section 5.3.2 in writing $s=\operatorname{Im} \omega, \lambda=\operatorname{Im} k$, and where erfe is the complementary error function. The results for $v^{*}, \lambda^{*}$ and $D$, obtained by solving (5.109) together with the saddle point condition $\partial s / \partial \lambda=s /\left.\lambda\right|_{\lambda *}$ numerically, are shown in Fig. 14 .

Other examples of equations with memory kernels can be found, e.g., in [103,104].

\subsubsection{Exact results for numerical finite difference schemes}

The fact that our results also apply to finite difference equations has the important implication that if we study a PDE with pulled fronts numerically using a finite difference approximation with grid size $\Delta x$ and timestep $\Delta t$, we can calculate $v^{*}(\Delta x, \Delta t)$ as well as $v(t ; \Delta x, \Delta t)$ exactly. This allows us to estimate analytically the intrinsic discretization error in these quantities, and hence to decide beforehand which grid and step size are needed to obtain a given accuracy.

As a first illustration, suppose that one integrates the F-KPP equation (1.1) numerically with an explicit Euler scheme. This amounts to approximating the PDE by

$$
\frac{u_{j}(t+\Delta t)-u_{j}(t)}{\Delta t}=\frac{u_{j+1}(t)-2 u_{j}(t)+u_{j-1}(t)}{(\Delta x)^{2}}+u_{j}(t)-u_{j}^{k}(t) .
$$




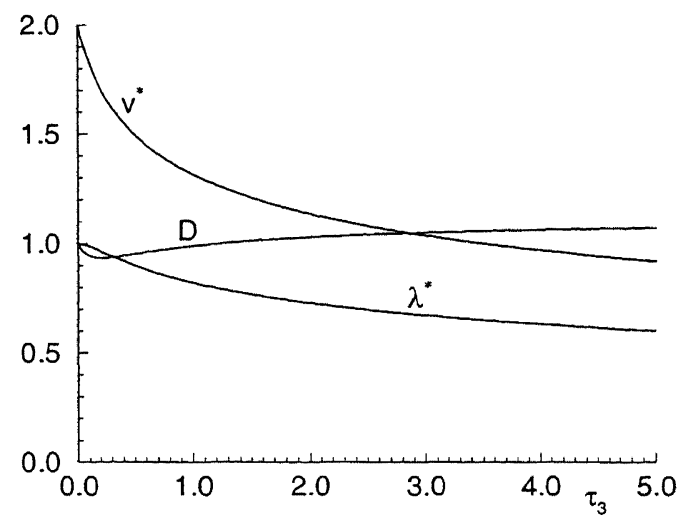

Fig. 14. Plot of $v^{*}, \lambda^{*}$, and $D$ as a function of $\tau_{3}$ for the extension (5.107) of the F-KPP equation with a memory kernel (5.108).

Upon substitution of $u_{j}(t) \sim \exp \{s t-\lambda x\}, x=j \Delta x$ into the linearized equation (we again follow the notation of Section 5.3 .2 by writing $s=\operatorname{Im} \omega, \lambda=\operatorname{Im} k$ ), we obtain

$$
\frac{\exp \{s \Delta t\}-1}{\Delta t}=1+\left(\frac{\sinh \frac{1}{2} \lambda \Delta x}{\frac{1}{2} \Delta x}\right)^{2},
$$

which is straightforward to solve for $s(\lambda ; \Delta x, \Delta t)$. As we emphasized above, by solving the saddle point condition $\partial s / \partial \lambda=s /\left.\lambda\right|_{\lambda^{*}}=v^{*}$, we can obtain the exact values of $v^{*}, \lambda^{*}$ and $D$ for any step and grid size, and in this way determine the accuracy of the numerical scheme. In general, these equations have to be solved by a simple numerical iteration routine, but for small $\Delta x$ and $\Delta t$, the result can easily be calculated analytically: expanding in $\Delta x$ and $\Delta t$, we find the dispersion relation

$$
s(\lambda ; \Delta x, \Delta t)=1+\lambda^{2}+\frac{1}{12} \lambda^{4}(\Delta x)^{2}-\frac{1}{2}\left(1+\lambda^{2}\right)^{2} \Delta t+\cdots .
$$

For $\Delta t \rightarrow 0, \Delta x \rightarrow 0$, this reduces to the continuum result $s(\lambda)=1+\lambda^{2}$, as it should. For the saddle point parameters, we find

$$
\begin{aligned}
& v^{*}=2-2 \Delta t+\frac{1}{12}(\Delta x)^{2}+\cdots, \\
& \text { Euler }: \quad \lambda^{*}=1+\Delta t-\frac{1}{8}(\Delta x)^{2}+\cdots, \\
& D=1-4 \Delta t+\frac{1}{2}(\Delta x)^{2}+\cdots .
\end{aligned}
$$

In practice, the Euler scheme is not used very often, because it is numerically very unstable and not very accurate. We have done all our simulations in Section 4 and in this section with a more stable and accurate semi-implicit method [105], ${ }^{24}$ which for the F-KPP equation amounts to the discretization

$$
\begin{aligned}
\frac{u_{j}(t+\Delta t)-u_{j}(t)}{\Delta t}= & \frac{1}{2}\left[\frac{u_{j+1}(t)-2 u_{j}(t)+u_{j-1}(t)}{(\Delta x)^{2}}\right] \\
& +\frac{1}{2}\left[\frac{u_{j+1}(t+\Delta t)-2 u_{j}(t+\Delta t)+u_{j-1}(t+\Delta t)}{(\Delta x)^{2}}\right]+\frac{1}{2}\left[u_{j}(t)+u_{j}(t+\Delta t)\right] \\
& -\frac{1}{2}\left[2 u_{j}^{k}(t)+k u_{j}^{k-1}(t)\left(u_{j}(t+\Delta t)-u_{j}(t)\right)\right] .
\end{aligned}
$$

\footnotetext{
${ }^{24}$ For the diffusion term, this method amounts to the Crank-Nicholson scheme, see, e.g., Section 17.2 of [118].
} 
The last term is obtained by expanding $u_{j}^{k}(t+\Delta t)$ about $u_{j}^{k}(t)$ to first order in $u_{j}(t+\Delta t)-u_{j}(t)$, so that one obtains a linear equation for the $u_{j}(t+\Delta t)$. This expansion makes what would otherwise have been an implicit method, into a semi-implicit method. This difference, however, does not matter for the leading edge dynamics evaluated below.

The dispersion relation is now given by

$$
\frac{\tanh \frac{1}{2} s \Delta t}{\frac{1}{2} \Delta t}=1+\left(\frac{\sinh \frac{1}{2} \lambda \Delta x}{\frac{1}{2} \Delta x}\right)^{2},
$$

which immediately yields $s(\lambda ; \Delta x, \Delta t)$. For small $\Delta x$ and $\Delta t$, the result is

$$
s(\lambda ; \Delta x, \Delta t)=1+\lambda^{2}+\frac{1}{12} \lambda^{4}(\Delta x)^{2}+\frac{1}{12}\left(1+\lambda^{2}\right)^{3}(\Delta t)^{2}+\cdots .
$$

For this integration scheme, it is now straightforward to find

$$
\begin{aligned}
& v^{*}=2+\frac{2}{3}(\Delta t)^{2}+\frac{1}{12}(\Delta x)^{2}+\cdots, \\
& \text { Semi-implicit : } \quad \lambda^{*}=1-\frac{2}{3}(\Delta t)^{2}-\frac{1}{8}(\Delta x)^{2}+\cdots, \\
& D=1+3(\Delta t)^{2}+\frac{1}{2}(\Delta x)^{2}+\cdots .
\end{aligned}
$$

We stress that these are the exact expressions for the application of this numerical scheme to the nonlinear diffusion equation, scaled to the normal form (2.1) and (2.2). They are therefore the "ideal" finite difference correction terms in the absence of numerical instabilities, round-off errors, etc. The correctness and accuracy of the prediction (5.117) for $v^{*}$ is demonstrated in Section 4 in Fig. 8(b).

We finally note that an early example of pulled front relaxation observed in a finite difference equation in space and time was seen in a mean-field model of ballistic growth [22]. In this paper, the prefactor of the $1 / t$ term, obtained by plotting $v$ versus $1 / t$, was found to be about $9 \%$ too small. Presumably, this discrepancy is due to the corrections from the $1 / t^{3 / 2}$ term: according to $(5.70)$, the term $\left(1-\left(\pi /\left(\lambda^{*^{2}} D t\right)\right)^{1 / 2}\right)$ generally gives rise to a lowering of the effective slope in a $v$ versus $1 / t$ plot, as Fig. 7 (b) clearly demonstrates.

\section{Summary and outlook}

\subsection{Summary of the main results}

The essential result of this paper is that for front propagation into unstable states, starting from steep initial conditions, the convergence of front velocity and shape is given in the pulled regime by the universal expressions

$$
\begin{aligned}
& v(t)=v^{*}+\dot{X}(t), \\
& \dot{X}(t)=-\frac{3}{2 \lambda^{*} t}\left(1-\left(\frac{\pi}{\left(\lambda^{*}\right)^{2} D t}\right)^{1 / 2}\right)+O\left(\frac{1}{t^{2}}\right), \\
& \phi=\Phi_{v(t)}\left(\xi_{X}\right)+O\left(\frac{1}{t^{2}}\right), \quad \text { for } \xi_{X} \lesssim \sqrt{t}, \\
& \xi_{X}=x-v^{*} t-X(t),
\end{aligned}
$$

provided the asymptotic front profile is uniformly translating. All terms in the expression for $v(t), \lambda^{*}, v^{*}$ and $D$ are given explicitly in terms of the dispersion relation of dynamical equation, linearized about the unstable state (see 
Eqs. (5.16), (5.17) and (5.19) or Table 5). These results are also summarized in Table 2. The dependence on pushing or pulling and on the initial conditions is sketched in Table 4.

With universal we mean that not only the asymptotic profile is unique, but also the relaxation towards it, provided we start with sufficiently steep initial conditions which decay exponentially faster than $\mathrm{e}^{-\lambda^{*} x}$ for $x \rightarrow \infty$. Moreover, the relaxation is universal in that it is independent of the precise nonlinearities in the equation, and of the precise form of the equation: it holds for PDEs, sets of PDEs, difference-differential equations, equations with memory kernels, etc., provided fronts are pulled and that the asymptotic front solution is uniformly translating, and provided that we are not at the bifurcation point from the pulled to the pushed regime, or at the bifurcation point $D=0$ towards pattern forming fronts (e.g., at $\gamma=\frac{1}{12}$ in the EFK-equation). The fact that the results also apply to finite difference equations has a nice practical consequence: if a PDE is studied numerically using a finite difference approximation scheme, both $v^{*}$ and the prefactors of the algebraic relaxation terms can also be calculated exactly for the numerical scheme. This allows one to estimate in advance how big step and grid sizes need to be, in order to achieve a particular numerical accuracy (see Section 5.6.6).

The remarkable relaxation properties are reminiscent of the universal corrections to scaling in critical phenomena, if we think of the relaxation as the approach to a unique fixed point in function space along a unique trajectory. An alternatively way to express this in more mathematical terms is to say that we have constructed the center manifold for front relaxation in the pulled regime.

The above expressions contain all universal terms: those of order $t^{-2}$ depend on the precise initial conditions and on the nonlinearities in the equations. The order of the limits is important here: our results are the exact expressions in a $1 / t$ expansion, i.e., when we take the large time limit while tracking the velocity of a particular fixed value of $\phi$. To order $1 / t^{2}$, this is equivalent to keeping $\xi_{X}$ fixed. When we interchange the limits by taking $\xi_{X}$ large at fixed time, there is a crossover to a different intermediate asymptotic regime for $\xi_{X} \gtrsim \sqrt{t}$. The different dynamical regions of a pulled front are sketched in Fig. 2.

The slow algebraic convergence of pulled fronts to the asymptotic velocity has important consequences, as it prohibits the derivation of a standard moving boundary approximation for patterns in more than one dimension that consist of propagating pulled fronts whose width is much smaller than their radius of curvature [37].

While we have limited the analysis in this paper to equations that admit uniformly translating front solutions, it turns out that most elements of our analysis can be extended to pattern forming fronts for which $\operatorname{Re} k^{*} \neq 0$ and $\operatorname{Re} \omega^{*} \neq 0$. In this case, the expression (6.2) with $1 / \sqrt{D}$ replaced by $\operatorname{Re}(1 / \sqrt{D})$ applies $[72,73,99]$.

In addition to our derivation of the above expressions for the convergence of pulled fronts, we have reformulated and extended the connection between front selection and the stability properties of fronts. This leads to an essentially complete picture also of front relaxation in the pushed regime and in the case of leading edge dominated dynamics resulting from flat initial conditions. For an interpretation of these results, again a consideration of the different dynamical regions of a front as in Fig. 2 is helpful. The relaxation behavior in the pulled regime with sufficiently steep initial conditions cannot be obtained simply from the properties of the stability operator of the pulled front solution, and therefore had to be obtained along a different route, which is summarized below.

\subsection{Summary of the main conceptual steps of the analysis}

The derivation of our central result on pulled front relaxation is based on the following steps:

1. From the dispersion relation $\omega(k)$ or from the characteristic function $S(k, \omega)$, we obtain $v^{*}, \lambda^{*}$ and $D$ (see Table 5). 
2. The double root condition which determines $v^{*}$ and $\lambda^{*}$ implies that the asymptotic large $\xi$ behavior of uniformly translating front solutions is as $\Phi^{*}(\xi)=(\alpha \xi+\beta) \mathrm{e}^{-\lambda^{*} \xi}$, where generically $\alpha \neq 0$.

3. The double root condition which determines $v^{*}$ and $\lambda^{*}$ also implies that the lowest order spatial derivative term in the dynamical equation for the leading edge representation $\psi=\mathrm{e}^{\lambda^{*} \xi} \phi(\xi, t)$ is of the diffusion type, $D \partial^{2} \psi / \partial \xi^{2}$ (see Sections 5.3.3, 5.4 and 5.5).

4. The diffusion type dynamics implied by 3 shows that in the comoving frame $\xi=x-v^{*} t$, the front profile shifts back with the collective coordinate $X(t)$ which grows logarithmically in time. Linearization about the asymptotic front solution $\Phi^{*}(\xi)$ in the $\xi$ frame is therefore impossible (see Sections 3.1.3 and 3.1.4). Instead, we introduce the frame $\xi_{X}=x-v^{*} t-X(t)$ with the expansion $\dot{X}(t)=c_{1} / t+c_{3 / 2} / t^{3 / 2}+\cdots$ and the corresponding leading edge transformation $\psi\left(\xi_{X}, t\right)=\mathrm{e}^{\lambda^{*} \xi_{X}} \phi(x, t)$.

5. In the front interior, the long-time expansion for $\dot{X}$ generates an expansion for the corrections to the front profile in inverse powers of $t$. To order $t^{-2}$ temporal derivatives of the front corrections do not come in, so that to this order the equations for the profile shape reduce to those for $\Phi_{v(t)}$. This immediately leads to (6.3) for the time dependence of the front profile.

6. In the leading edge, where nonlinearities can be neglected, we use an asymptotic expansion for $\psi\left(\xi_{X}, t\right)$, linearized about $\psi=0$, in terms of functions of the similarity variable $z=\xi_{X}^{2} /(4 D t)$ of the diffusion equation. Now for small values of $z$, the expansion has to match the boundary condition $\psi \approx \mathrm{e}^{-\lambda^{*} \xi_{X}} \Phi^{*}\left(\xi_{X}\right) \approx \alpha \xi_{X}+\beta$ (implied by observation 2), and for large $\xi_{X}$, the terms in the (intermediate) asymptotic expansion have to decay as a Gaussian $\mathrm{e}^{-z}=\exp \left\{-\xi_{X}^{2} /(4 D t)\right\}$ times a polynomial in the similarity variable $z$. These two requirements fix the constants $c_{1}, c_{3 / 2}, \ldots$ in the expansion of $\dot{X}$, and hence (6.2).

\subsection{Open problems}

What one considers as remaining open problems concerning pulled front propagation, will depend largely on one's background and standards regarding the desired mathematical rigor. While our results are exact and yield an almost complete understanding of the general mechanism of pulled front propagation, they have, of course, not been derived rigorously. In physics, such a situation is often not just quite acceptable but even quite gratifying, but more mathematically inclined readers may wish to take up the challenge to provide a more rigorous justification. More work could also be done on enlarging the classes of equations for which the assumptions underlying our approach can be shown to hold, i.e., for which one can show that fronts are pulled and that there exists a family of uniformly translating front solutions.

Within the realm of our approach, one can consider slight extensions of our method to two non-generic special cases. First of all, we have focused on the case of sufficiently steep initial conditions such that the steepness $\lambda=-\lim _{x \rightarrow \infty} \ln \phi(x, 0)$ is larger than $\lambda^{*}$. As we discussed at the end of Section 3 , the intermediate case in which for large $x, \phi(x, 0) \simeq x^{-\nu} \mathrm{e}^{-\lambda^{*} x}$ with $v<2$, does give a $\nu$-dependent result for the coefficient of the $1 / t$ term. According to Bramson [74], the next order correction is of order $1 /(t \ln t)$. This suggests that for this special case logarithmic terms will have to be included in the expansion. Second, at the bifurcation point from uniformly translating solutions to pattern forming fronts, which in the EFK equation (5.91) happens at $\gamma=\frac{1}{12}$, the diffusion coefficient $D$ vanishes (see Eq. (5.92)). At this bifurcation point, the equation for the leading edge representation $\psi$ is not of the diffusion type, so our asymptotic expansion breaks down right at this point. We have not investigated what happens then.

As mentioned before, we will elsewhere address what we consider the most interesting remaining challenges, the extension of (part of) these results to pattern forming and chaotic fronts [72,73,99] and the question whether weakly curved fronts can be analyzed with a moving boundary approximation [37], an issue which is of central importance for understanding fronts in two and three dimensions like streamers [15]. 
6.4. The multiplicity of front solutions and of solutions of the saddle point equations

As we discussed in Section 5.2, our general discussion of the convergence of pulled fronts to their asymptotic velocity and shape is based on the assumption that a uniformly translating front solution $\Phi^{*}(\xi)$ exists (see (5.19) for a definition), and that it is a member of a one-parameter family of front solutions. What happens if this family of front solutions does not exist has, to our knowledge, not been investigated systematically for real equations. However, experience with various pattern generating fronts - especially with a similar case in which no generalized uniformly translating solutions exist in the quintic complex Ginzburg-Landau equation [66], even though the dynamics is pulled - yields the scenario that the leading edge just spreads according to the linearized equations, and that the front interior "just follows", in the sense that if there are uniformly translating front solutions, the front interior and the region behind it relax smoothly, while if there are none, it is forced to follow the spreading in some other way. This leads one to conjecture that if there is no family of uniformly translating front solutions, the velocity relaxation will still be described by Eqs. (6.1) and (6.2) in the leading edge, but that in the interior front region the dynamics will be inherently time-dependent, e.g., incoherent [73].

This can occur in particular in the following situation: as mentioned in Section 5.3.1, it can happen that the dispersion relation is such that there is more than one allowed non-trivial solution for the equations for $v^{*}$ and $\lambda^{*}$. According to the linearized equation, arbitrary, sufficiently steep initial conditions will spread out asymptotically with the largest speed $v^{*}$. Hence the asymptotic spreading speed of pulled fronts emerging from steep initial conditions is simply the largest velocity $v^{*}$. Now, according to a counting argument for the multiplicity of uniformly translating front solutions, the multiplicity of front solutions associated with different solutions of the saddle point equations for $v^{*}$ will differ: if there are two solutions $v_{1}^{*}$ and $v_{2}^{*}$ with $\lambda_{1}^{*}<\lambda_{2}^{*}$, the multiplicity of front solutions with velocity near $v_{2}^{*}$ and an asymptotic spatial decay rate near $\lambda_{2}^{*}$ will be smaller than that of those with velocity near $v_{1}^{*}$ and a spatial decay rate near $\lambda_{1}^{*}$. Investigations of the issue of the competition between various solutions $v^{*}$ will therefore also bear on the issue raised in the beginning of this section, the question what happens when there is no uniformly translating solution $\Phi^{*}$. In particular, the dynamics in an equation that has a family of uniformly translating fronts associated with the solution $v_{1}^{*}$, should show a transition from smoothly relaxing interior dynamics for $v_{1}^{*}>v_{2}^{*}$ to incoherent interior dynamics for $v_{1}^{*}<v_{2}^{*}$.

\subsection{A step-by-step guideline for applying these results}

If one just wants to apply our results to a given dynamical equation with a given initial condition without worrying about the derivation and justification, one can simply follow the following guidelines:

1. Linearize the dynamical equation about the unstable state, and determine the characteristic equation $S(k, \omega)=0$ for modes $\exp \{-\mathrm{i} \omega t+\mathrm{i} k x\}$ in the linearized equation.

2. Solve the double root or saddle point conditions from Table 5 to determine $v^{*}, k^{*}$ and $D$.

3. Check whether the leading edge of the initial conditions is steeper than $\mathrm{e}^{-\lambda^{*} x}$ with $\operatorname{Im} k^{*}=\lambda^{*}$. Only then the front is a candidate for pulling with an asymptotic velocity $v^{*}$.

4. Check whether the conditions (5.19) under which fronts are expected to be uniformly translating, $\operatorname{Re} k^{*}=$ $\operatorname{Re} \omega^{*}=0, \operatorname{Im} D=0$ are satisfied. If not, the fronts will be pattern generating rather than uniformly translating (see Section 6.4 above).

5. Assuming the conditions under 4 are obeyed, so that the asymptotic front is expected to be uniformly translating, investigate by a counting argument or otherwise whether there is a one-parameter family of uniformly translating front profiles $\Phi_{v}(\xi)$ that includes $\Phi^{*}(\xi)$.

6. Determine, by using bounds, comparison theorems or physical arguments, whether the fronts will be pushed or pulled. This determines, which particular regime from Table 4 applies. 
7. If according to points 4-6 there is a family of front solutions that include $\Phi^{*}$, and if the dynamics is pulled, then our predictions (6.1)-(6.3) or Table 2 apply. If the conditions under 4 are satisfied but there is no family of uniformly translating solutions according to 5, then our formula (6.2) should apply but one can then expect intrinsic non-trivial dynamics in the front interior to remain, so that (6.3) does not apply. If 4 is not satisfied (as for the EFK equation (5.91) for $\gamma>\frac{1}{12}$ ), one expects pattern generating fronts with a similar algebraic convergence (see $[72,73,99])$.

\subsection{The subtle role of the nonlinearities: an alternative intuitive explanation}

As we have seen in (2.46) and (5.20), the convergence of the linear spreading velocity to the asymptotic value $v^{*}$ is as $v(t)=v^{*}-1 /\left(2 \lambda^{*} t\right)+\cdots$, while the convergence of nonlinear fronts is as $v(t)=v^{*}-3 /\left(2 \lambda^{*} t\right)+\cdots$. The prefactor of the $1 / t$ in the latter case is just three times larger than for the linear spreading velocity. What is this subtle difference due to?

In this paper, we have attributed the difference to the presence of the term $\alpha \xi$ in the large- $\xi$ asymptotics $(\alpha \xi+\beta) \mathrm{e}^{-\lambda^{*} \xi}$ of $\Phi^{*}(\xi)$. We used an argument closely related to the one presented below, to prove in Section 2.5.2, that $\alpha \neq 0$. The functional form of $\Phi^{*}$ leads to the requirement that the leading term in the expansion in similarity solutions in the leading edge is $\left(\xi / t^{3 / 2}\right) \exp \left\{-\xi^{2} /(4 D t)\right\}$, not $(1 / \sqrt{t}) \exp \left\{-\xi^{2} /(4 D t)\right\}$ (see Section 3.1.1). Nevertheless, one may want to have a better intuitive understanding of why the asymptotics of the linear spreading velocity is not correct for the nonlinear front relaxation - after all, one might at first sight think that the linear spreading results should be correct sufficiently far forward in the leading edge, where the nonlinearities can be neglected. The following picture allows us to understand why this is wrong, and why the same type of algebraic convergence also applies to pattern forming and chaotic fronts [72,73,99].

Consider for simplicity the F-KPP equation (1.1). As discussed in the introduction and Section 2.5.2, the dynamical equation for the leading edge representation $\psi(\xi, t)$ of $\phi$ is

$$
\partial_{t} \psi(\xi, t)=\partial_{\xi}^{2} \psi(\xi, t)-\psi^{3}(\xi, t) \mathrm{e}^{-2 \lambda^{*} \xi}
$$

We can think of the nonlinear $\psi^{3} \mathrm{e}^{-2 \lambda^{*} \xi}$ term as a localized sink term in the diffusion equation for $\psi$ : the term vanishes for positive $\xi$ due to the exponential term, and for large negative $\xi$ since $\psi$ vanishes exponentially in the region to the left where $\phi$ saturates (see (2.54)). Thus, if we think of $\psi$ as representing the density of diffusing particles, then in the region where this term is non-vanishing it describes the annihilation of particles. For the half space to the right of it, where the particles freely diffuse, this term therefore acts like an absorbing boundary on the left. This is actually all that remains of the nonlinearities in the equation! Whenever the integrated sink strength $\alpha$ (the spatial integral of the nonlinear term, in agreement with (2.54)) is non-zero, the problem in the leading edge reduces to that of the build-up of a diffusion field in the presence of an absorbing boundary (and at the same time, as (2.54) shows, $\alpha \neq 0$ ). In this language, the pulled to pushed transition occurs precisely when the absorption strength $\alpha$ vanishes, and indeed precisely at this point the velocity convergence is as $v(t)=v^{*}-1 /\left(2 \lambda^{*} t\right)+\cdots$ (see Eq. (3.66)).

There is one complication: unlike the usual problems of diffusion in the presence of a given absorbing boundary, the "sink" in (6.5) depends on the relaxing field $\psi$ itself. In fact, as we discussed extensively in the paper, the diffusive dynamics of $\psi$ leads to a logarithmic shift of the sink in time, in the frame $\xi$. That is why in this interpretation we have to go, for self-consistency, to the frame $\xi_{X}=\xi-X(t)$. In this frame, the "sink" or "absorbing wall" remains essentially fixed in time, and so the dynamics of $\psi$ is, in leading order, that of a diffusion field in the presence of a fixed absorbing wall. As it is well known, in such a case a linear gradient $\psi \propto \xi_{X}$ will build-up in front of the wall, to balance the constant annihilation of particles in the wall region. 
Clearly, even if the "sink" strength is not stationary in time, the build-up of the linear diffusion gradient far ahead of it will not be affected. The present interpretation therefore yields a natural starting point for analyzing the velocity relaxation of non-uniformly translating fronts. This will be explored elsewhere $[72,73,99,102]$.

We end this paper by stressing that while we have shown that nonlinear fronts relax according to the " $\frac{3}{2}$ law" $v(t)=v^{*}-3 /\left(2 \lambda^{*} t\right)+\cdots$, one cannot apply this result completely with closed eyes. An amusing illustration of this warning is the following. It has been noted that the spreading velocity in the equation

$$
\frac{\partial \phi}{\partial t}=\frac{\partial^{2} \phi}{\partial x^{2}}+\phi+e\left(\frac{\partial \phi}{\partial x}\right)^{2}
$$

follows the " $\frac{1}{2}$ law" $v(t)=v^{*}-1 /\left(2 \lambda^{*} t\right) \cdots=2-1 /(2 t)+\cdots[106,119]$. At first sight, this equation therefore might appear to yield a counterexample to our assertions. In fact, it does not. Our results only hold for equations where the growth of the dynamical field saturates behind the front, not in the case in which the growth is unbounded. If the growth is unbounded, our arguments for why $\alpha \neq 0$, and hence for the " $\frac{3}{2}$ law", break down. The above equation is precisely an example in which the growth does not saturate: for $e>0$ and positive $\phi$, the nonlinear term only increases the growth. Hence there is no saturation and the spreading velocity $v_{\mathrm{nl}}(t)$ in the presence of the nonlinearities is larger than the one of the linear equation: $v_{\mathrm{nl}}(t) \geq v^{*}-1 /(2 t)+\cdots$. Apparently, in practice the equality is obeyed asymptotically. Of course, if we add a saturation term of the type $-\phi^{k}$ with $k>1$ to the RHS of (6.6), we obtain regular fronts and our usual expression for $v(t)$ is recovered.

\section{Acknowledgements}

The work described in this paper started from discussions with C. Caroli. The work of UE was supported by the Dutch Science Foundation (NWO) and the EU-TMR-network "Patterns, Noise, and Chaos".

\section{Appendix A. An upper bound for $v_{c}$ in the nonlinear diffusion equation}

With a generalization of the leading edge transformation introduced in Section 2.6, it is straightforward to prove the well-known upper bound $v_{\mathrm{c}} \leq v_{\text {sup }}$, where

$$
v_{\text {sup }}=2 \sup _{0 \leq \phi \leq 1}\left(\frac{f(\phi)}{\phi}\right)^{1 / 2}
$$

for the selected front velocity in the nonlinear diffusion equation, if the initial conditions have steepness $\lambda>\frac{1}{2} v_{\text {sup }}$. The steepness $\lambda$ of a front is defined in (2.6). To prove this bound, transform (2.1) to a frame $\xi=x-v t$, and write

$$
\psi(\xi, t)=\exp \left\{\frac{1}{2} \nu \xi\right\} \phi(x, t)
$$

The equation of motion is now

$$
\partial_{t} \psi=\partial_{\xi}^{2} \psi-\frac{1}{4} v^{2} \psi+\exp \left\{\frac{1}{2} v \xi\right\} f\left(\psi \exp \left\{-\frac{1}{2} v \xi\right\}\right) .
$$

If the initial steepness is $\lambda>\frac{1}{2} v$, then

$$
\lim _{\xi \rightarrow \pm \infty} \psi(\xi, t)=0 \quad \text { for all } 0 \leq t<\infty
$$


since the steepness of the leading edge $(\xi \rightarrow \infty)$ is conserved for all finite times, cf. the discussion in Section 2.5; and since convergence at $\xi \rightarrow-\infty$ is guaranteed by $\phi \rightarrow 1$ behind the front together with the transformation (A.2). Thus the decay of $\psi$ at $\xi \rightarrow \pm \infty$ is exponential in $\xi$ for $t<\infty$. Hence, the whole equation can be multiplied by $\psi$ and integrated over $\xi$. This yields

$$
\partial_{t} \int_{\xi} \frac{\psi^{2}}{2}=-\int_{\xi}\left\{\left(\partial_{\xi} \psi\right)^{2}+\psi^{2}\left[\frac{v^{2}}{4}-\frac{f\left(\psi \exp \left\{-\frac{1}{2} v \xi\right\}\right)}{\psi \exp \left\{-\frac{1}{2} v \xi\right\}}\right]\right\},
$$

where all integrals are finite. The RHS of this equation is strictly negative, if $v>v_{\text {sup }}$ (A.1). Therefore, in a frame moving with velocity $v>v_{\text {sup }}$, the integral $\int_{\xi} \psi^{2}$ decays in time. This means, that the frame is propagating too rapidly, so that the front shrinks away in the leading edge representation $\psi$ (A.2). Only a frame moving with velocity $v \leq v_{\text {sup }}$ can propagate along with the speed of the front. $v_{\text {sup }}$ is therefore an upper bound for the asymptotic velocity of any initial condition with $\lambda>\frac{1}{2} v_{\text {sup. }}$.

For nonlinearity $f_{\mathrm{KPP}}=\phi-\phi^{k}$, we have $v_{\text {sup }}=2$. But on the other hand, we know (see Section 2 ) that $v_{\text {sup }} \geq v_{\mathrm{c}} \geq v^{*}=2$. Hence, these fronts are pulled with $v_{\mathrm{c}}=v^{*}=2$. For nonlinearity $f_{\epsilon}=\epsilon \phi+\phi^{n+1}-\phi^{2 n+1}$, we have $v_{\text {sup }}=(1+4 \epsilon)^{1 / 2}>2 \sqrt{\epsilon}=v^{*}$.

This version of the argument for $v<v_{\text {sup }}[72]$ can be generalized for equations with higher spatial derivatives, forming both uniformly translating fronts or pattern forming fronts $[73,99]$.

\section{Appendix B. The generalized nonlinear diffusion equation}

Analyze a general equation with first temporal and second spatial derivative:

$$
F\left(\phi, \partial_{x} \phi, \partial_{x}^{2} \phi, \partial_{t} \phi\right)=0
$$

A front translating uniformly with velocity $v$ solves

$$
F\left(\Phi_{v}, d_{\xi} \Phi_{v}, d_{\xi}^{2} \Phi_{v},-v d_{\xi} \Phi_{v}\right)=0, \quad \xi=x-v t .
$$

The stability analysis of such a solution and the further treatment of convergence is identical with what we did for the nonlinear diffusion equation (1.1) in Sections 2 and 3. We only need to transform the linear operators as discussed below. Our analysis is directly relevant for the equation studied in [107].

We use the definition of functional derivatives as in (5.32)-(5.36). A linear perturbation $\eta(\xi, t)(2.25)$ about a uniformly translating state $\Phi_{v}$ then solves the linear equation $\partial_{t} \eta=\mathcal{L}_{v} \eta(2.26)$, respectively, (5.30) with the linear operator being now

$$
\mathcal{L}_{v}=f_{2}(\xi) \partial_{\xi}^{2}+f_{1, v}(\xi) \partial_{\xi}+f_{0}(\xi), \quad f_{1, v}=v+f_{1} .
$$

For transforming to a Schrödinger problem $\partial_{t} \psi=\mathcal{H}_{v} \psi+\mathrm{o}\left(\psi^{2} \mathrm{e}^{-\alpha}\right), \mathcal{H}_{v}=-\partial_{y}^{2}+V_{v}(y)$, we now have to make the coefficient of the first order derivative $\partial_{\xi}$ vanish, and the coefficient of the second-order derivative $\partial_{\xi}^{2}$ constant. This can be achieved through a transformation similar to (2.28) and (2.29), combined with a nonlinear transformation $y(\xi)$ of the lengthscale $\xi$ :

$$
\begin{aligned}
& \psi=\mathrm{e}^{\alpha} \eta, \quad \mathrm{d} \alpha(\xi)=\frac{2 f_{1, v}-\partial_{\xi} f_{2}}{4 f_{2}} \mathrm{~d} \xi \\
& \mathcal{H}_{v}(y)=-\mathrm{e}^{\alpha(\xi)} \mathcal{L}_{v} \mathrm{e}^{-\alpha(\xi)}=-\partial_{y}^{2}+V_{v}(y),
\end{aligned}
$$




$$
\begin{aligned}
& \mathrm{d} y(\xi)=\frac{\mathrm{d} \xi}{\left(f_{2}(\xi)\right)^{1 / 2}}\left(\Leftrightarrow \partial_{y}=\sqrt{f_{2}(\xi)} \partial_{\xi}\right), \\
& V_{v}(y(\xi))=\frac{f_{1, v}^{2}-4 f_{0} f_{2}}{4 f_{2}}+\frac{f_{2} d_{\xi} f_{1, v}-f_{1, v} d_{\xi} f_{2}}{2 f_{2}}+\frac{3\left(d_{\xi} f_{2}\right)^{2}-4 f_{2} d_{\xi}^{2} f_{2}}{16 f_{2}} .
\end{aligned}
$$

We use again the convention $\lim _{\xi \rightarrow \infty} \Phi_{v}(\xi)=0$. By construction, the pulled velocity $v^{*}$ is the velocity, where $V_{v^{*}}(\infty)=0$. Accordingly, now

$$
v^{*}=2 \sqrt{f_{0}(\infty) f_{2}(\infty)}-f_{1}(\infty)
$$

The steepness of the leading edge is

$$
\lambda^{*}=\left.\partial_{\xi} \alpha\right|_{\xi \rightarrow \infty, v=v^{*}}=\left(\frac{f_{0}(\infty)}{f_{2}(\infty)}\right)^{1 / 2}
$$

(In the convention of Section 5.2: $f_{n}(\infty)=c_{n}$.) In the leading edge region, the relation between $y$ and $\xi$ is linear: $y=\xi /\left(f_{2}(\infty)\right)^{1 / 2}$.

If $V_{v^{*}}(y) \geq 0$ for all $y$, there are no destablizing linear modes within the Hilbert space of (B.5). Then the front propagating with $v^{*}$ is stable. The remaining analysis translates from Sections 2 and 3 step-by-step with only the explicit form of the linear operators $\mathcal{L}_{v}$ and $\mathcal{H}_{v}$ and the transformation operator $\mathrm{e}^{\alpha}$ being more involved.

If there is a range of $y$ such that $V_{v^{*}}(y)$ becomes negative, there might be a destabilizing mode in the spectrum of linear perturbations. In this case, there must be a pushed front solution with some velocity $v^{\dagger}>v^{*}$ with steepness $\lambda=\lambda_{+}\left(v^{\dagger}\right)>\lambda_{0}\left(v^{\dagger}\right)=\frac{1}{2} v^{\dagger}$. Such a pushed front might even be integrated analytically, if one can find an analytic solution $\psi(\phi)$ of

$$
F\left(\phi, \psi, \psi \frac{\mathrm{d} \psi}{\mathrm{d} \phi},-v \psi\right)=0,
$$

equivalent to (C.3). $\xi(\phi)$ can then be integrated as in (C.5). (Again, a closed form for $\psi(\phi)$ cannot be found for pulled fronts, except possibly for equations at the pushed/pulled transition.)

\section{Appendix C. Analytical solutions for pushed nonlinear diffusion fronts and transition to pulling}

We here discuss, how to find analytical solutions for uniformly translating fronts $\phi(\xi)$ in the equation

$$
\partial_{\xi}^{2} \phi+v \partial_{\xi} \phi+f(\phi)=0
$$

- We rephrase and straighten the method from [65] (see also [108,109]) how to find analytical front solutions.

- We recall that analytical solutions can be found only for pushed fronts (propagating either into a meta- or into an unstable state, Cases I and II from Table 4), but not for pulled fronts (Case IV).

- We recall that only a strongly heteroclinic orbit, i.e., a front approaching $\phi=0$ with $\lambda>\lambda_{0}(v)$, is a candidate for a pushed front. This allows us to calculate the critical $\epsilon$ for the pushed/pulled transition in the case of the nonlinearity (1.10).

Write the equation as a flow in phase space as in (2.24)

$$
\partial_{\xi}\left(\begin{array}{c}
\psi \\
\phi
\end{array}\right)=\left(\begin{array}{c}
-v \psi-f(\phi) \\
\psi
\end{array}\right),
$$


where $\xi$ parametrizes the flow. If $\phi$ is monotonic in $\xi, \psi$ can be parametrized by $\phi$ instead of by $\xi$. This substitution yields for $\psi(\phi)$

$$
\psi \frac{\partial \psi}{\partial \phi}+v \psi+f(\phi)=0
$$

This is the differential equation for the trajectory in phase space, where now the translational degree of freedom is removed together with the parametrization $\xi$ of the flow. The resulting differential equation is one order lower than the original differential equation (C.1). According to (2.16), the initial condition for the integration at $\phi \approx 1$ is

$$
\psi(\phi=1-\delta)=-\tilde{\lambda}_{-} \delta+o\left(\delta^{2}\right), \quad \tilde{\lambda}_{-}=\frac{1}{2} v-\left(\frac{1}{4} v^{2}-f^{\prime}(1)\right)^{1 / 2},
$$

so the front trajectory is unique and can be integrated. In some cases, the integration can be done analytically, if one is lucky enough to find an analytical solution $\psi(\phi)$ of Eq. (C.3) for a given $f(\phi)$. If we have a solution $\psi(\phi)$, then the function $\xi(\phi)$ can be integrated as

$$
\xi=\int_{\phi(0)}^{\phi(\xi)} \frac{\mathrm{d} \phi}{\psi(\phi)} .
$$

The final step consists in finding the inverse function $\phi=\phi(\xi)$, if this is possible.

Note now that solutions $\psi(\phi)$ can be found analytically only, if $\phi$ approaches $\phi=0$ with a single exponential $\phi \propto \mathrm{e}^{-\lambda \xi}$, since only then $\psi(\phi)$ has the simple analytic form $\psi(\phi)=-\lambda \phi+o\left(\phi^{2}\right)$ at $\phi \rightarrow 0$. Any other form of the approach to $\phi=0$, cf. (2.17), would not be expressible in a simple analytic expression for $\psi(\phi)$. In particular, a generic $\Phi^{*}$ front with $\Phi^{*} \alpha(\alpha \xi+\beta) \mathrm{e}^{-\lambda^{*} \xi}$ in the leading edge does not have a simple analytical expression for $\psi(\phi)$ since $\psi(\phi)=-\lambda^{*} \phi+\alpha \phi /(\alpha \xi+\beta)+\mathrm{o}\left(\phi^{2}\right)$, so a pulled front generically cannot be integrated analytically. Again $\alpha \neq 0$ spoils the conventional tools of analysis!

Given an analytical front solution with velocity $v$ and decay rate $\lambda$, one has to check the nature of the front. A pushed front is a strongly heteroclinic front, i.e., it has leading edge steepness $\lambda=\lambda_{+}(v)>\lambda_{0}(v)$. (For the notation of $\lambda$ 's, compare Eq. (2.20).) If $\lambda=\lambda_{0}(v)=\lambda_{ \pm}(v)$, we have found a front at the transition point from pushed to pulled with leading edge behavior $\phi \propto \mathrm{e}^{-\lambda^{*} \xi}$. This is the only pulled front, we can integrate. If $\lambda_{-}=\lambda_{-}(v)<\lambda_{0}(v)$, we have a particular flat front, that has evolved from an initial condition with the same flatness in the leading edge.

Finding analytical solutions for pushed fronts can even be turned into a machinery, if we do not fix $f$ and look for a $\psi$, but if we define $\psi(\phi)$ and then calculate $f(\phi)$. For

$$
\psi=-\lambda \phi\left(1-\phi^{n}\right),
$$

we calculate, e.g.,

$$
f(\phi)=\lambda(v-\lambda) \phi+\lambda(\lambda(n+2)-v) \phi^{n+1}-\lambda^{2}(n+1) \phi^{2 n+1}=\bar{\epsilon} \phi+\phi^{n+1}-(1+\bar{\epsilon}) \phi^{2 n+1},
$$

where we have to identify $v=(n+2) \lambda-1 / \lambda$, and $\bar{\epsilon}=\lambda(v-\lambda)$. The analytic front solution for (C.6) can be calculated from (C.5) and inverted to yield

$$
\phi(\xi)=\left[1+\left(\phi(0)^{-n}-1\right) \mathrm{e}^{\lambda n \xi}\right]^{-1 / n} .
$$

This solution is a pushed front, if $\lambda \geq \lambda_{0}(v)=\frac{1}{2} v$, which implies $\bar{\epsilon} \leq 1 / n$. For such $\bar{\epsilon}$, we find pushed fronts with decay rate $\lambda=((\bar{\epsilon}+1) /(n+1))^{1 / 2}$, velocity $v=(1+\bar{\epsilon}(n+2)) /((n+1)(1+\bar{\epsilon}))^{1 / 2}$, and analytical form (C.8). For $\bar{\epsilon}=1 / n$, the solution is a front on the transition point from pushed to pulled fronts with asymptotic decay $\phi \propto \mathrm{e}^{-\lambda^{*} \xi}+\mathrm{o}\left(\phi^{2}\right)$. For $\bar{\epsilon}>1 / n$, the solution (C.8) is a flat front evolving from flat initial conditions. Fronts evolving from sufficiently steep initial conditions are then pulled, propagate with velocity $2 \bar{\epsilon}^{1 / 2}$, have decay rate $\lambda^{*}=\bar{\epsilon}^{1 / 2}$ and no analytic form of the front solution can be found. 
Eq. (1.1) with nonlinearity (C.7) can also be rescaled to bring the equation to the more familiar form

$$
\begin{aligned}
& \partial_{\tau} \varphi=\partial_{y}^{2} \varphi+\epsilon \varphi+\varphi^{n+1}-\varphi^{2 n+1}, \\
& \epsilon=\bar{\epsilon}(1+\bar{\epsilon}), \quad(1+\bar{\epsilon})=\frac{t}{\tau}=\left(\frac{x}{y}\right)^{2}=\left(\frac{\varphi}{\phi}\right)^{n} .
\end{aligned}
$$

This reproduces precisely the form of the nonlinearity (1.10) with the stable state now at $\varphi_{\mathrm{s}}=\left[\frac{1}{2}\left(1+(1+4 \epsilon)^{1 / 2}\right)\right]^{1 / n}$. Accordingly, the critical $\epsilon$ is now $\epsilon_{\mathrm{c}}=(n+1) / n^{2}$. Fronts propagate for $\epsilon<\epsilon_{\mathrm{c}}$ with the pushed velocity $v^{\dagger}=$ $\left[(n+2)(1+4 \epsilon)^{1 / 2}-n\right] /\left[2(n+1)^{1 / 2}\right]$ and decay rate $\lambda_{+}\left(v^{\dagger}\right)=\left[1+(1+4 \epsilon)^{1 / 2}\right] /\left[2(n+1)^{1 / 2}\right]$. For $\epsilon>\epsilon_{\mathrm{c}}$, they propagate with the pulled velocity $v^{*}=2 \epsilon^{1 / 2}$ and decay rate $\lambda^{*}=\epsilon^{1 / 2}$.

\section{Appendix D. Linear stability analysis of moving front solutions}

In this appendix, we study the linear perturbations $\eta$ of a uniformly translating front $\Phi_{v}(\xi)$ in the nonlinear diffusion equation. The problem is defined in Eqs. (2.25)-(2.27) and can be transformed to a Schrödinger problem (2.28)-(2.31) with linear operator $\mathcal{H}_{v}$. Since $\mathcal{H}_{v}$ is self-adjoint, we can decompose functions that lie in the Hilbert space of $\mathcal{H}_{v}$, into the orthonormal set of eigenfunctions of $\mathcal{H}_{v}$. Eigenfunctions in this Hilbert space form a complete set. However, it is obvious that not all linear perturbations with $|\eta| \ll 1$ are in this space: only perturbations with

$$
\lim _{\xi \rightarrow \infty}|\eta| \mathrm{e}^{\lambda_{0}(v) \xi}<\infty \quad \text { with } \quad \lambda_{0}(v)=\frac{1}{2} v
$$

can lie in the Hilbert space (which consists of square integrable functions and of solutions proportional to plane waves $\mathrm{e}^{\mathrm{i} k \xi}$ as $\left.\xi \rightarrow \pm \infty\right)$.

\section{D.1. Schrödinger stability analysis}

The general properties of the spectrum and eigenfunctions of $\mathcal{H}_{v}$ within the Hilbert space can be immediately obtained from a few well-known results which to physicists are known from quantum mechanics (see, e.g., [86]), since $\mathcal{H}_{v}$ is the Hamiltonian operator for a (quantum) wave in a potential in one dimension. The potential is asymptotically lower on the right than on the left, since

$$
V(\infty)=\frac{1}{4} v^{2}-f^{\prime}(0)<\frac{1}{4} v^{2}-f^{\prime}(1)=V(-\infty)
$$

according to (2.2). If we write the temporal behavior of an eigenfunction as $\tilde{\psi}_{\sigma}(\xi) \mathrm{e}^{-\sigma t}$, one finds that the spectrum of

$$
\mathcal{H}_{v} \tilde{\psi}_{\sigma}=\sigma \tilde{\psi}_{\sigma}
$$

is continuous for $\sigma \geq V(\infty)$, and that the eigenfunctions are distributions, i.e., essentially plane waves $\mathrm{e}^{\mathrm{i} k \xi}$ with $k= \pm(\sigma-V(\infty))^{1 / 2}$ as $\xi \rightarrow \infty$. One immediately concludes that a front $\Phi_{v}$ with velocity $v<v^{*}=2\left(f^{\prime}(0)\right)^{1 / 2}$ will be unstable against the continuous spectrum of linear perturbations with "energies" $V(\infty)<\sigma<0$.

For a front $\Phi_{v}$ with velocity $v \geq v^{*}=2\left(f^{\prime}(0)\right)^{1 / 2}$, there still might be a point spectrum of bound and square integrable states with $\sigma<0$. Bound states have a finite number of nodes, and there is a one-to-one correspondence between the number of nodes and the eigenvalue of the bound state "wave function" $\psi_{\sigma}$ : the eigenfunction with the lowest eigenvalue $\sigma$ is nodeless (if it exists), the eigenfunction corresponding to the next largest bound state eigenvalue has one node, etc. Therefore, the point spectrum is bounded from below by the "energy" $\sigma$ of the nodeless 
eigenfunction, if it exists. Now, one eigenfunction is known: the translation mode $\tilde{\psi}_{0}$ clearly has $\sigma=0$. It can be generated by an infinitesimal translation of $\Phi_{v}$ :

$$
\tilde{\psi}_{0}=\mathrm{e}^{\lambda_{0}(v) \xi} \partial_{\xi} \Phi_{v}, \quad \mathcal{H}_{v} \tilde{\psi}_{0}=0 .
$$

If $\Phi_{v}$ is monotonic, $\tilde{\psi}_{0}$ will be nodeless. If $\Phi_{v}$ is non-monotonic, $\tilde{\psi}_{0}$ will have nodes.

From this one might be tempted to immediately draw conclusions on the stability of monotonic or non-monotonic front solutions. However, this is only possible if $\tilde{\psi}_{0}$ is in the Hilbert space! Comparison with (2.17) shows, that this is the case, if either $v=v^{*}$ and $\alpha=0$, or if $v>v^{*}$ and $A_{v}=0$, i.e., for one of the strongly heteroclinic orbits.

If a front $\Phi_{v}$ obeys one of these conditions and if it is monotonic, then $\tilde{\psi}_{0}$ is the eigenfunction in the Hilbert space with the lowest "energy" $\sigma=0$. Therefore all other eigenfunctions will have $\sigma>0$ and will decay in time as $\mathrm{e}^{-\sigma t}$. An arbitrary linear perturbation in the Hilbert space can be decomposed into the complete set of eigenfunctions, and therefore it will decay too (apart of course from the non-decaying translation mode $\tilde{\psi}_{0}$ ).

If such a front $\Phi_{v}$ is non-monotonic it will have $n$ extrema, with $n>0$ some integer. The translation mode then has $n$ nodes, and hence there are then $n$ bound eigenfunctions $\tilde{\psi}_{\sigma}$ with negative $\sigma$. The front profile is then linearly unstable with respect to these modes. Since any generic initial condition will have a non-vanishing contribution from these destabilizing modes, a non-monotonic $\Phi_{v}$ will generically not be approached for long times. Such a $\Phi_{v}$ is called dynamically unstable.

The analysis of the spectrum and eigenfunctions of the Schrödinger operator in the Hilbert space therefore yields the following results:

1. A front $\Phi_{v}$ with velocity $v<v^{*}$ is intrinsically unstable against a continuous band of linear perturbations from the Hilbert space. Such a front generically will not be approached under the dynamics.

2. A front $\Phi^{*}$ with velocity $v=v^{*}$ and $\alpha=0$ is unstable against perturbations from the Hilbert space, if it is non-monotonic, and it is stable, if it is monotonic. There is a continuous band of linear perturbations with $\sigma \geq 0$ that continuously extends down to $\sigma=0$. Accordingly, there is no gap in the excitation spectrum, which already hints at the non-exponential convergence towards a monotonic $\Phi^{*}$.

3. A strongly heteroclinic orbit $\Phi_{v}$ with $v>v^{*}$ and $A_{v}=0$, if it exists, is unstable against perturbations from the Hilbert space, if it is non-monotonic, and it is stable, if it is monotonic. If strongly heteroclinic orbits exist, by construction (see Section 2.2) only the one with the largest velocity $v=v^{\dagger}$ is monotonic, and the front $\Phi^{*}$ with velocity $v=v^{*}<v^{\dagger}$ is non-monotonic and thus unstable. So only for $\Phi^{\dagger}$, the spectrum of linear perturbations is purely positive: $\sigma \geq 0$. For $\Phi^{\dagger}$ there is at best a discrete spectrum of linear perturbations in the Hilbert space in the range $0<\sigma<V(\infty)=\frac{1}{4}\left(v^{\dagger^{2}}-v^{*^{2}}\right)$, and the continuous spectrum begins at $\sigma \geq V(\infty)$. Convergence of all perturbations in the Hilbert space will thus be exponential in time like $\mathrm{e}^{-\sigma t}$, with $\sigma$ the smallest positive eigenvalue.

Note the restrictions of this analysis:

1. Up to now, we have no predictions for fronts with velocity $v \geq v^{*}$, whose translation mode $\tilde{\psi}_{0}$ (D.4) is outside the Hilbert space. We will see that the equivalence of stability and monotonicity extends beyond the Hilbert space analysis.

2. The analysis of general initial conditions might require linear perturbations, that lie outside the Hilbert space, even if $\tilde{\psi}_{0}$ is in the Hilbert space.

\section{D.2. Linear perturbations outside the Hilbert space}

The mapping to the Schrödinger problem is a powerful method for perturbations $\eta$ about a front $\Phi_{v}$, that lie within the Hilbert space, because we then can work with a complete set of orthogonal functions. In general, however, this space of perturbations needs to be completed by functions from outside the Hilbert space. 
To see this, consider for simplicity an initial condition, that is close to some $\Phi_{v}$ with $v \geq v^{*}$, but steeper than this asymptotic front: $\lim _{x \rightarrow \infty} \phi(x, 0) / \Phi_{v}(x)=0$. Then the steepness in the leading edge of $\eta=\phi-\Phi_{v}$ will be dominated by $\Phi_{v}$, and

$$
\psi=\eta \mathrm{e}^{\lambda_{0}(v) \xi} \stackrel{\xi \rightarrow \infty}{\sim} \begin{cases}\alpha \xi+\beta & \text { for } v=v^{*} \\ \mathrm{e}^{-\mu(v) \xi} & \text { for } v=v^{\dagger} \text { or generally, for } v>v^{*} \text { and } A_{v}=0 \\ \mathrm{e}^{\mu(v) \xi} & \text { for } v>v^{*} \text { and } A_{v} \neq 0 .\end{cases}
$$

with $\mu(v)=\left(\frac{1}{4} v^{2}-1\right)^{1 / 2}>0$ from (2.17). Accordingly, only for a pushed front propagating with velocity $v=v^{\dagger}$ (or more generally for a strongly heteroclinic orbit with $v>v^{*}$ and $A_{v}=0$ ) or for a pulled front with velocity $v^{*}$ and $\alpha=0$, the linear perturbation $\eta \mathrm{e}^{\lambda_{0}(v) \xi}$ is in the Hilbert space of $\mathcal{H}_{v}$. The decay of the zero mode $\tilde{\psi}_{0}$ (D.4) is asymptotically the same as that of $\psi$ in (D.5). So a treatment of linear perturbations outside the Hilbert space is clearly called for.

In general, we want to decompose perturbations $\eta$ that obey

$$
\lim _{\xi \rightarrow \pm \infty}|\eta(\xi, t)| \ll 1
$$

This is required for the linearization of $\phi$ about $\Phi_{v}$ in (2.26). We aim at a decomposition of $\eta(\xi, t)$ into eigenfunctions $\eta_{\sigma}(\xi) \mathrm{e}^{-\sigma t}$. We therefore return to the eigenvalue equation for such an eigenmode, which according to (2.26) and (2.27) is given by

$$
\left[\partial_{\xi}^{2}+v \partial_{\xi}+f^{\prime}\left(\Phi_{v}(\xi)\right)+\sigma\right] \eta_{\sigma}=0 .
$$

Our previous analysis in the Hilbert space already has identified many of these eigenmodes, in fact all those, which obey (D.1). This criterium on $\eta_{\sigma}$ is too strict at $\xi \rightarrow \infty$, so we now need to additionally analyze perturbations with $\mathrm{e}^{-\lambda_{0}(v) \xi}<\left|\eta_{\sigma}(\xi)\right|<1$ as $\xi \rightarrow \infty$, which lie outside the Hilbert space. On the other hand, for $\xi \rightarrow-\infty$, Eq. (D.1) is less restrictive than (D.6). This gives us the freedom to impose only $\left|\eta_{\sigma}(\xi)\right| \lesssim \mathrm{e}^{\lambda_{0}(v)|\xi|}$ as $\xi \rightarrow-\infty$, since such a divergence can be compensated for by perturbations from inside the Hilbert space, where we make use of its completeness. We therefore now impose the boundary conditions

$$
\lim _{\xi \rightarrow \infty}\left|\eta_{\sigma}(\xi)\right|<\infty, \quad \lim _{\xi \rightarrow-\infty} \mathrm{e}^{\lambda_{0}(v) \xi}\left|\eta_{\sigma}(\xi)\right|<\infty
$$

where perturbations that additionally obey $\mathrm{e}^{-\lambda_{0}(v) \xi}\left|\eta_{\sigma}(\xi)\right|<\infty$ as $\xi \rightarrow \infty$, are in the Hilbert space of $\mathcal{H}_{v}$.

First of all, we note that the translation mode $\eta_{0}(\xi)=\partial_{\xi} \Phi_{v}(\xi)$ (D.4) now is always included in the larger space (D.8) of perturbations.

Second, solve (D.7) for $\xi \rightarrow \infty$ and find in analogy to (2.17) that

$$
\eta_{\sigma}(\xi)=A_{\sigma} \mathrm{e}^{-\Lambda-\xi}+B_{\sigma} \mathrm{e}^{-\Lambda_{+} \xi},
$$

with

$$
\Lambda_{ \pm}(\sigma, v)=\frac{1}{2} v \pm\left(\frac{1}{4} v^{2}-f^{\prime}(0)-\sigma\right)^{1 / 2} .
$$

For brevity of notation, we here allowed $\Lambda_{ \pm}(\sigma, v)$ to be complex. In Fig. 15, we plot $\Lambda_{ \pm}$versus $\sigma$, both for the case of a front propagating into an unstable state $\left(f^{\prime}(0)>0\right)$, and for the case of a front between a stable and a metastable state $\left(f^{\prime}(0)<0\right)$, and for $f^{\prime}(0)>0$, we furthermore distinguish between $v>v^{*}$ and $v=v^{*}$. The leading edge solution (D.9), of course, precisely coincides with the leading edge behavior of the Hilbert space functions, except that one case was excluded from the Hilbert space: a leading edge with $A_{\sigma} \neq 0$ and $\sigma \leq V(\infty)=\frac{1}{4} v^{2}-f^{\prime}(0)$ does 

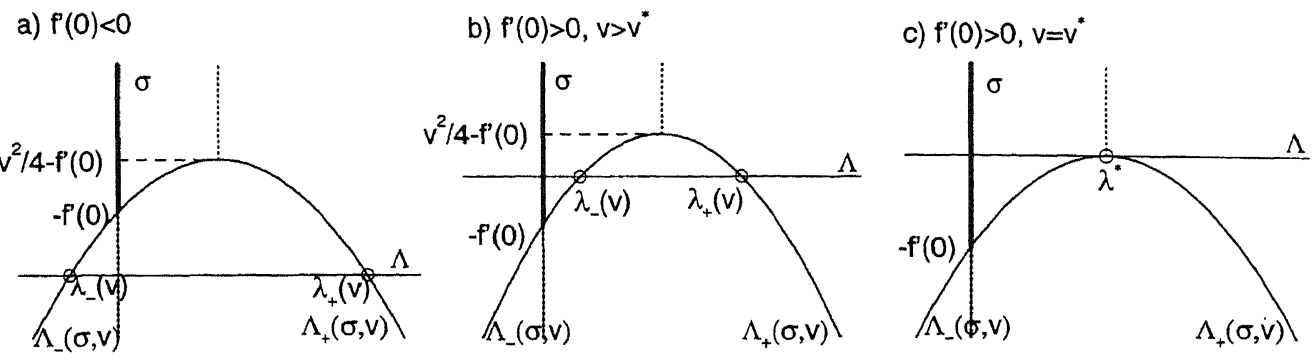

Fig. 15. Steepness $\Lambda(\sigma, v)$ (D.10) versus decay rate $\sigma$ of linear perturbations $\eta_{\sigma}$ ((D.7) and (D.8)) of a given front $\Phi_{v}$ with velocity $v \geq v^{*}$ The solid curve denotes real $\Lambda$, the dotted curve denotes the real part of complex $\Lambda . \lambda_{ \pm}(v)$ and $\lambda^{*}$ are the steepnesses of $\Phi_{v}$ and of the zero mode $\eta_{0}=\partial_{\xi} \Phi_{v}$. They are marked by circles on the $\Lambda$-axis. The generic steepness of a front $\Phi_{v}$ with $v>v^{*}$ is $\lambda_{-}(v)$, while in the particular case of $A_{v}=0$, it is $\lambda_{+}(v)$. The continuous spectrum of $\sigma$ is denoted by a fat solid line on the $\sigma$-axis, the interval in which there may be discrete eigenvalues $\sigma$ by the fat dotted line. The continuous spectrum within the Hilbert space of $\mathcal{H}_{v}$ exists only at $\frac{1}{4} v^{2}-f^{\prime}(0) \leq \sigma$. The continuous spectrum for $-f^{\prime}(0)<\sigma<\frac{1}{4} v^{2}-f^{\prime}(0)$ is on the $\Lambda_{-}$-branch. There might be discrete solutions characterized by $A_{\sigma}=0$. They lie on the $\Lambda_{+}$-branch, might exist for all $\sigma<\frac{1}{4} v^{2}-f^{\prime}(0)$, and need to be constructed: (a) the front $\Phi_{v}$ propagates into a metastable state $\left(f^{\prime}(0)<0\right)$. Its steepness is $\lambda_{+}(v)$. It is stable against all linear perturbations with $\Lambda<\lambda_{+}(v)$. The discrete spectrum of steep perturbations with $\Lambda>\lambda_{+}(v)$ needs to be investigated; (b) the front propagates into an unstable state $\left(f^{\prime}(0)>0\right)$ with velocity $v>v^{*}$. It is stable against all linear perturbations with $\lambda_{-}(v)<\Lambda<\lambda_{+}(v)$, it is unstable against the continuous spectrum of very flat perturbations with $0<\Lambda<\lambda_{-}(v)$, which might be excluded by the initial conditions. The discrete spectrum of steep perturbations with $\Lambda>\lambda_{+}(v)$ needs to be investigated; (c) the front propagates into an unstable state $\left(f^{\prime}(0)>0\right)$ with velocity $v=v^{*}$. The discussion is as for (b) after identifying $\lambda_{ \pm}\left(v^{*}\right)=\lambda^{*}$.

not obey the boundary condition (D.1). It does obey the boundary condition (D.6), if $\sigma \geq-f^{\prime}(0)$. Let us therefore now focus on the additional perturbations with

$$
-f^{\prime}(0)<\sigma \leq V(\infty)=\frac{1}{4} v^{2}-f^{\prime}(0) .
$$

If $A_{\sigma} \neq 0$, such perturbations are outside the Hilbert space, but they do obey (D.8).

Are there such perturbations for a given $\sigma$, and how many? For answering this question we need to analyze $\eta_{\sigma}$ globally, in close analogy to the global analysis of the $\Phi_{v}$ as a function of $v$ in Section 2.2. Solving (D.7) at $\xi \rightarrow-\infty$ yields two exponents

$$
\tilde{\Lambda}_{ \pm}(\sigma, v)=\frac{1}{2} v \pm\left(\frac{1}{4} v^{2}-f^{\prime}(1)-\sigma\right)^{1 / 2}=\lambda_{0}(v) \pm(V(-\infty)-\sigma)^{1 / 2},
$$

in analogy with (2.15) and (2.16). Since $V(-\infty)>V(\infty)$ (D.3), for $\sigma \leq V(\infty)$ we certainly have $V(-\infty)-\sigma>0$. The coefficient of $\mathrm{e}^{-\tilde{\Lambda}_{+}(\sigma, v) \xi}$ therefore needs to vanish for $\eta_{\sigma}$ to obey (D.8). Behind the front for $\xi \rightarrow-\infty$, we therefore find that

$$
\eta_{\sigma}(\xi)= \pm \mathrm{e}^{-\tilde{\Lambda}_{-}\left(\xi-\xi_{0}\right)}+o\left(\mathrm{e}^{-2 \tilde{\Lambda}_{-} \xi}\right)
$$

for an $\eta_{\sigma}$ obeying (D.8) and (D.11). Eq. (D.13) determines $\eta_{\sigma}$ uniquely because the arbitrary constant coefficient $\pm \mathrm{e}^{\tilde{\Lambda}-\xi_{0}}$ can be scaled out of a linear equation like (D.7). Such a linear equation can always be integrated towards $\xi \rightarrow \infty$, where it uniquely determines the coefficients $A_{\sigma}$ and $B_{\sigma}$ in (D.9). Accordingly, $A_{\sigma}$ and $B_{\sigma}$ generically are non-vanishing, in complete analogy to the argument for $A_{v}$ and $B_{v}$ in (2.17) to be generically non-vanishing in $\Phi$.

What do we gain with these extra solutions? The eigenfunctions in the Hilbert space had a continuous spectrum for $\sigma \geq V(\infty)=\frac{1}{4}\left(v^{2}-v^{*^{2}}\right) \geq 0$ and at best a discrete spectrum defined by $A_{\sigma}=0$ for $\sigma<V(\infty)$. Adding the solutions, that obey (D.8), we extend the continuous spectrum down to $\sigma \geq-f^{\prime}(0)=V(\infty)-\frac{1}{4} v^{2}<0$ and find at best a discrete spectrum defined by $A_{\sigma}=0$ for $\sigma<-f^{\prime}(0)$. These discrete solutions for $\sigma<-f^{\prime}(0)$ all lie in the Hilbert space. 
Let us now look at the steepness in the leading edge of the solutions outside the Hilbert space. They have a $\sigma$ from the interval (D.11), and $A_{\sigma} \neq 0$. For these we observe (cf. Fig. 15) that

$$
\begin{array}{llll}
\Lambda_{-}(\sigma, v)>\lambda_{-}(v) & \text { for } \quad \sigma>0 & \text { (decaying), } \\
\Lambda_{-}(\sigma, v)<\lambda_{-}(v) & \text { for } \quad \sigma<0 & \text { (destabilizing), } \\
\Lambda_{-}(0, v)=\lambda_{-}(v) & \text { for } \quad \sigma=0 & \text { (marginal), }
\end{array}
$$

with $\lambda_{-}(v)$ from (2.16). This means, that these linear eigenmodes $\eta_{\sigma}$ of $\Phi_{v}$ will decay $(\sigma>0)$, if they are steeper than $\mathrm{e}^{-\lambda_{-}(v) \xi}$, and that they will destabilize a front $\Phi_{v}$, if they are flatter. Note that the spectrum of decaying modes is continuous down to zero, as $\sigma \downarrow 0$ as $\Lambda_{-} \downarrow \lambda_{-}$.

It is tempting to conclude here immediately, that a front $\Phi_{v}(\xi)$ with velocity $v \geq v^{*}$ will be stable against all perturbations, which are steeper in the leading edge than $\mathrm{e}^{-\lambda_{-}(v) \xi}$. However, the possible existence of the discrete set of solutions with $A_{\sigma}=0$ and $\sigma<0$ requires special attention, since these perturbations are steeper than $\mathrm{e}^{-\lambda_{0}(v) \xi}$, but destabilizing $(\sigma<0)$. Now, if $\Phi_{v}$ is strongly heteroclinic $\left(A_{v}=0\right)$, we already found in Section D.1, that such destabilizing perturbations exist, if and only if $\Phi_{v}$ is non-monotonic. We now need to show that this argument also holds for fronts $\Phi_{v}$ with $v>v^{*}$ and $A_{v} \neq 0$ or for fronts $\Phi^{*}$ with velocity $v^{*}$ and $\alpha \neq 0$. The following five steps (i)-(v) prove this: (i) Impose (D.13) at $\xi \rightarrow-\infty$. This defines a unique solution of Eq. (D.7) for $\eta_{\sigma}$ for every $\sigma<V(-\infty)$. In fact, we only need to analyze $\sigma<V(\infty)$, since we know the spectrum for larger $\sigma$. (ii) Integrate (D.7) forward towards $\xi \rightarrow \infty$ for a very large negative $\sigma$. The variation of $f^{\prime}\left(\Phi_{v}(\xi)\right)$ in space then can be almost neglected. Therefore at $\xi \rightarrow \infty$, we will find (D.9) with $\left|A_{\sigma} / B_{\sigma}\right| \gg 1$. For our further construction it is crucial to observe that such a perturbation for sufficiently large negative $\sigma$ will be nodeless. It does not matter, on the other hand, that this solution typically will not obey our bound (D.8), since we only use it as a means for constructing the solutions with $A_{\sigma}=0$, which will not only obey (D.8), but even lie inside the Hilbert space. (iii) Upon increasing $\sigma$ continuously, at discrete values of $\sigma<V(\infty), \eta_{\sigma}$ will gain an extra node. Since the generation of every new node is associated with a change of sign of the perturbation at $\xi \rightarrow \infty$, if the sign at $\xi \rightarrow-\infty$ is kept fixed, the appearance of an additional mode can only occur at a $\sigma$, where the sign of $A_{\sigma}$ changes. (iv) We know the number of nodes of the zero mode $\eta_{0}$. It is identical to the number of extrema of $\Phi_{v}$. We therefore know the number of particular perturbations with $A_{\sigma}=0$ and $\sigma<0$. (v) From this it follows that if $\Phi_{v}$ is monotonic, there are no particular perturbations with $A_{\sigma}=0$ and $\sigma<0$. If $\Phi_{v}$ is non-monotonic, there are such perturbations.

In Section 2.2, we have counted the multiplicity of front solutions $\Phi_{v}$ as a function of $v$. Here we have counted the multiplicity of perturbations $\eta_{\sigma}$ of a front $\Phi_{v}$ as a function of $\sigma$. This counting was based on the proper asymptotics of the solutions at $\xi \rightarrow \pm \infty$, which is of the same structure for both $\Phi_{v}$ and $\eta_{\sigma}$, so the counting argument follows exactly the same lines in both cases.

The conclusions from this appendix are summarized in Section 2.3.

\section{Appendix E. Stability analysis, selection and rate of convergence}

In this appendix, we analyze the implications of the results of the stability analysis of Appendix D for understanding the selection of fronts and for the rate of convergence towards the asymptotic front solution. For pushed fronts, the stability analysis implies that the relaxation towards the pushed front solution is exponentially fast, while for pulled fronts the spectrum is gapless, and the convergence cannot be obtained from the stability spectrum.

\section{E.1. Pushed regime: $v_{\mathrm{c}}=v^{\dagger}$}

We first consider equations with the nonlinearity $f(\phi)$ such that the slowest stable front is a strongly heteroclinic orbit in phase space with $A_{v \dagger}=0$ in (2.17). We have denoted this asymptotic front with $\Phi^{\dagger}$ and its velocity with 
$v^{\dagger}$. Its steepness is $\lambda_{+}\left(v^{\dagger}\right)=\lambda_{0}\left(v^{\dagger}\right)+\mu\left(v^{\dagger}\right)$, cf. (2.23). There is a continuous family of stable front solutions $\Phi_{v}$ with velocity $v>v^{\dagger}$ which are all flatter than $\lambda_{-}\left(v^{\dagger}\right)=\lambda_{0}\left(v^{\dagger}\right)-\mu\left(v^{\dagger}\right)$. Their steepness $\lambda=\lambda_{-}\left(v^{\dagger}\right)$ is related to their velocity $v$ through

$$
v(\lambda)=\lambda+\frac{1}{\lambda},
$$

as can be obtained by inverting (2.18).

Case I. Consider an initial condition with steepness $\lambda_{\text {init }}>\lambda_{0}\left(v^{\dagger}\right)$. We let $\phi$ evolve sometime, and then linearize it about $\Phi^{\dagger}$. According to (2.37), the perturbation $\eta$ will have steepness $\lambda_{\eta}>\lambda_{0}\left(v^{\dagger}\right)$. It then is in the Hilbert space analyzed in Section D.1. We can decompose the perturbation into the known eigenperturbations. The spectrum of decay rates has no negative eigenvalues, one eigenvalue zero and then a gap above zero. A contribution from the zero mode can be made vanishing by adjusting the position of the subtracted asymptotic front $\Phi_{v}$, by making use of the translational freedom of $\Phi_{v}$. The perturbation then can be decomposed into Hilbert space functions $\eta_{\sigma}$ with $\sigma$ all positive and bounded away from zero. Thus, for large times the perturbation will decay exponentially. This means that an initial condition with $\lambda_{\text {init }}>\lambda_{0}\left(v^{\dagger}\right)$ will converge to $\Phi^{\dagger}$ exponentially in time, generically with $\mathrm{e}^{-\sigma_{1} t}$, where $\sigma_{1}$ is the smallest positive eigenvalue.

Case II. If the initial steepness is $\lambda_{-}\left(v^{\dagger}\right)<\lambda_{\text {init }} \leq \lambda_{0}\left(v^{\dagger}\right)$, the perturbation of $\phi$ about $\Phi^{\dagger}$ will not be in the Hilbert space. However, we do know from the results illustrated in Fig. 15 that there is an eigenmode $\eta_{\sigma}$ of the linear stability operator of $\Phi^{\dagger}$ with the proper steepness $\lambda_{\text {init }}=\lambda_{\eta}$, that will decay exponentially in time (see Section D.2). The remaining linear perturbation $\eta-\eta_{\sigma}$ might lie in the Hilbert space, in which case we are back to Case I. If it does not, we have to identify the subleading $\lambda$, its corresponding eigenmode $\eta_{\sigma}$, etc. The iteration of this construction leads us to conclude that the perturbation indeed will decay exponentially in time. (Examples of exponential convergence towards pushed fronts which is dominated by such modes can be found in Fig. 19 of [65].) Another way of putting the argument is that only perturbations with $\lambda<\lambda_{-}\left(v^{\dagger}\right)$ can grow in time, but these cannot be involved in the decomposition of a perturbation with $\lambda_{\eta}>\lambda_{-}\left(v^{\dagger}\right)$. A more elegant way of analyzing this case and the following ones is discussed in Section 2.5.

Case III. If the initial steepness is $\lambda_{\text {init }}<\lambda_{-}\left(v^{\dagger}\right)$, and we linearize $\phi$ about $\Phi^{\dagger}$, there is a perturbation $\eta_{\sigma}$ with steepness $\lambda_{\eta}=\lambda_{\text {init }}$ that is growing in time $(\sigma<0)$. So such an initial condition cannot approach $\Phi^{\dagger}$ or any other asymptotic front $\Phi_{v}$ with steepness $\lambda_{\text {asympt }}>\lambda_{\text {init }}$. If we linearize $\phi$ about the asymptotic front $\Phi_{v}$ with the same steepness $\lambda_{\text {init }}=\lambda_{\text {asympt }}$, the remaining perturbation will be steeper, so contributions from the zero mode are excluded by construction, and the perturbation can be decomposed into eigenperturbations of $\Phi_{v}$, which all decay in time.

In summary: all initial conditions with $\lambda_{\text {init }}>\lambda_{-}\left(v^{\dagger}\right)$ converge exponentially in time to the "selected" front with velocity $v_{\text {sel }}=v^{\dagger}$ and steepness $\lambda_{\text {sel }}=\lambda_{+}\left(v^{\dagger}\right)$. Initial conditions with $\lambda_{\text {init }}<\lambda_{-}\left(v^{\dagger}\right)$ will converge to a quicker asymptotic front with steepness $\lambda_{\text {asympt }}=\lambda_{\text {init }}$ and velocity $v\left(\lambda_{\text {init }}\right)$ given by (E.1).

In Section 2.1, we have termed an initial condition sufficiently steep $\left(\lambda_{\text {init }}>\lambda_{\text {steep }}\right)$, if it approached the "selected" front for large times. We have denoted the steepness of the selected front with $\lambda_{\text {sel }}$. In the pushed regime, one can thus identify these parameters with

$$
\begin{array}{ll}
\lambda_{\text {steep }}=\lambda_{-}\left(v^{\dagger}\right)=\frac{1}{2} v^{\dagger}-\mu\left(v^{\dagger}\right), & \mu\left(v^{\dagger}\right)=\left(\frac{1}{4}\left(v^{\dagger}-4\right)\right)^{1 / 2}, \\
\lambda_{\text {sel }}=\lambda_{+}\left(v^{\dagger}\right)=\frac{1}{2} v^{\dagger}+\mu\left(v^{\dagger}\right), & v_{\text {sel }}=v^{\dagger} .
\end{array}
$$

\section{E.2. Fronts into metastable states}

The only difference between a pushed front propagating into an unstable state, i.e., with a nonlinearity $f$ such that $f^{\prime}(0)>0$ and $v_{\mathrm{c}}=v^{\dagger}>v^{*}$, and a front propagating into a metastable state, i.e., with $f^{\prime}(0)<0$, is the sign 
of $\lambda_{-}(v)$ : for a front into a metastable state, we have

$$
\mu(v)=\left(\frac{1}{4}\left(v^{2}-4 f^{\prime}(0)\right)\right)^{1 / 2}>\frac{1}{2} v \text { for } f^{\prime}(0)<0,
$$

so $\lambda_{-}(v)<0$ and $\lambda_{+}(v)>0$ for all $v>0$ (the sign of $\lambda_{0}(v)$ is the same as the sign of $v$ ). Suppose, that the selected front still travels with positive speed $v_{\text {sel }}=v^{\dagger}$ (otherwise reverse $x$ ). Because now $\lambda_{-}(v)<0$,

$$
\lambda_{\text {steep }}=0,
$$

so all initial conditions are sufficiently steep and converge to $\Phi^{\dagger}$. The continuous spectrum of asymptotic solutions $\Phi_{v}$ with $\lambda_{\text {asympt }}<\lambda_{\text {steep }}$ ceases to exist, and the asymptotic front $\Phi^{\dagger}$ therefore now is unique.

For the convergence of an initial condition $\phi$ towards $\Phi^{\dagger}$ we still need to distinguish whether $\lambda_{\text {init }}$ is larger or smaller than $\lambda_{0}\left(v^{\dagger}\right)=\frac{1}{2} v^{\dagger}$. If $\lambda_{\text {init }}>\lambda_{0}\left(v^{\dagger}\right)$, the perturbation about $\Phi^{\dagger}$ lies in the Hilbert space, while for $\lambda_{\text {init }}<\lambda_{0}\left(v^{\dagger}\right)$, it does not. This corresponds to the Cases I and II for $v_{c}=v^{\dagger}$ above, which apply literally. In both cases the initial conditions converge to $\Phi^{\dagger}$ exponentially in time. Case III does not occur for fronts into metastable states.

\section{E.3. Pulled regime: $v_{\mathrm{c}}=v^{*}$}

At the transition from fronts propagating into metastable towards fronts into unstable states, $f^{\prime}(0)$ changes sign, and so does $\lambda_{-}(v)$. At this point a continuum of possible attractors $\Phi_{v}$ of the dynamics comes into existence, but the convergence behavior of sufficiently steep initial conditions is completely unchanged. In other words: Cases I and II are completely unchanged and only Case III needs to be considered additionally for initial conditions with $\lambda_{\text {init }}<\lambda_{\text {steep. }}$.

A qualitative change in the convergence behavior of sufficiently steep initial conditions $\lambda_{\text {init }}>\lambda_{\text {steep }}$ only takes place at the transition from the pushed to the pulled regime. This happens for $f$ changing such that $v^{\dagger}$ approaches $v^{*}$. Then

$$
\lambda_{\text {steep }}=\lambda_{0}\left(v^{*}\right)=\lambda_{\text {sel }} .
$$

This transition leaves the multiplicity of possible attractors unchanged, but the resulting changes in the spectrum have deep consequences for the convergence behavior of sufficiently steep initial conditions.

We now need to distinguish but two cases for the initial condition, namely $\lambda \geq \lambda^{*}$ and $\lambda<\lambda^{*}$, where we use the short-hand notation $\lambda^{*}=\lambda_{0}\left(v^{*}\right)=\lambda_{ \pm}\left(v^{*}\right)=\frac{1}{2} v^{*}$.

For flat initial conditions $\lambda_{\text {init }}<\lambda^{*}$, the arguments from Case III above apply literally. Such an initial condition will approach a front $\Phi_{v}$ with velocity $v\left(\lambda_{\text {init }}\right)>v^{*}$ given by (E.1) and with steepness $\lambda_{\text {asympt }}=\lambda_{\text {init }}$. Sufficiently steep initial conditions, however, exhibit a new behavior.

Case IV. Consider a sufficiently steep initial condition with $\lambda>\lambda^{*}$. As before we linearize the profile $\phi(x, t)$ about the selected front $\Phi^{*}$ after a sufficient evolution time. The corresponding perturbation $\eta=\phi-\Phi^{*}$ then decays like $\Phi^{*}(2.21)$, because the steepness of $\phi(x, t)$ remains larger than that of $\Phi^{*}$ at any finite time $t$ (cf. Eq. (2.37)). As a result, $\eta$ is just outside the Hilbert space in the generic case of $\alpha \neq 0$ (2.17), just like the zero mode (D.4). The Hilbert space has a continuous spectrum for all decay rates $\sigma>0$, and there are no growing perturbations with $\sigma<0$. The perturbation $\eta$ can be written as a multiple of the zero mode $\eta_{0}$ plus a remainder inside the Hilbert space. From this we might be tempted to argue that the perturbation will decay, and that we only cannot tell how quickly - probably non-exponential, because the spectrum is gapless. However, in contrast to Cases I-III, there is no way to get rid of the zero mode, because no matter at which position $\xi_{0}$ one places the subtracted $\Phi^{*}\left(\xi-\xi_{0}\right), \Phi^{*}$ will always dominate the large $\xi$ behavior, and therefore the coefficient of the zero mode 
in the decomposition of the perturbation will always be non-vanishing. A convergence argument based on simply neglecting the contribution from the zero mode is bound to be wrong: in the very same way we could argue that a steep initial condition converges to $\Phi_{v}$ with just any $v \geq v^{*}$. Strictly speaking, the linear stability analysis does not even allow us to conclude that sufficiently steep initial conditions approach $\Phi^{*}$ at all. We only can reason that there is no steeper attractor than $\Phi^{*}$, and that one therefore expects that the pulled front solution $\Phi^{*}$ is selected from steep initial conditions. The different analytical tools that are developed in Section 2.5 to analyze the convergence behavior confirm this.

The results of this appendix are summarized in Table 4 (in Section 2).

\section{Appendix F. General integration of $g_{n / 2}^{\text {sp }}(z)$}

We here show how to find special solutions $g_{n / 2}^{\mathrm{sp}}(z)$ of inhomogeneous equations like (3.44) or (3.45) in general. The general form of such an equation is

$$
\hat{T}_{n}\left[z, d_{z}\right] g(z)=i_{n}(z),
$$

with $i_{n}(z)$ the inhomogeneity and $\hat{T}_{n}\left[z, d_{z}\right]$ the operator

$$
\hat{T}_{n}\left[z, d_{z}\right]=z \frac{\mathrm{d}^{2}}{\mathrm{~d} z^{2}}+\left(\frac{1}{2}-z\right) \frac{\mathrm{d}}{\mathrm{d} z}+\frac{1}{2} n \text {. }
$$

We search for a particular solution $g(z)$ of Eq. (F.1). A particular solution of the homogeneous equation $\left(i_{n}(z)=0\right)$ can be expressed by Hermite polynomials:

$$
\hat{T}_{n}\left[z, d_{z}\right] h_{n}(z)=0, \quad h_{0}(z)=1, \quad h_{1}(z)=\sqrt{2 z}, \quad h_{2}(z)=1-2 z, \text { etc. }
$$

The ansatz $g(z)=h_{n}(z) u_{n}(z)$ reduces (F.1) to an equation for $d_{z} u_{n}(z)$ of first order:

$$
\hat{T}_{n} g=z h_{n}(z)\left(\frac{\mathrm{d}}{\mathrm{d} z}+\frac{\mathrm{d} \ln h_{n}(z)}{\mathrm{d} z}+\frac{1-2 z}{2 z}\right) \frac{\mathrm{d} u_{n}(z)}{\mathrm{d} z}=z h_{n}(z) \frac{d_{z}\left(M_{n}(z) d_{z} u_{n}(z)\right)}{M_{n}(z)},
$$

where in the last line we introduced the integrating factor

$$
M_{n}(z)=h_{n}^{2}(z) \sqrt{z} \mathrm{e}^{-z} .
$$

Identify now $\hat{T}_{n} g_{n}=i_{n}$, integrate twice, and substitute $M_{n}$ by the full expression. A special solution of (F.1) then reads

$$
g(z)=h_{n}(z) \int_{a}^{z} \mathrm{~d} x \frac{\int_{b}^{x} \mathrm{~d} y i_{n}(y) h_{n}(y) \mathrm{e}^{-y} / \sqrt{y}}{h_{n}^{2}(x) \sqrt{x} \mathrm{e}^{-x}}
$$

where the integration constants $a$ and $b$ are free. If we in particular choose $b=\infty$, the integrated exponential $\exp \{x-y\}$ cannot exceed unity, and $g_{n}^{\mathrm{sp}}(z)$ can at most diverge algebraically, if the integrated inhomogeneity $i_{n}(z)$ is algebraic.

Integrating Eq. (3.55) for $g_{0}(z)$ as in (F.6) with $b=\infty$, we find for the algebraic divergence of $g_{0}(z)$ for large $z$ :

$$
g_{0}(z) \sim 3 \alpha z \ln z \quad \text { as } z \rightarrow \infty
$$

while the solution of the homogeneous equation diverges only as $h_{2}(z) \sim z$. For determining the small $z$ expansion of (F.6), it must be noted that the factor $h_{n}(x)^{-2}$ is singular at the zeroes of $h_{n}(x)$. Hence, (F.6) needs to be evaluated separately in each interval between the zeroes of $h_{n}(x)$. This can be done by a proper choice of $a$. It can be shown that the results in each interval join smoothly. 


\section{Appendix G. Algebraic convergence at the pushed/pulled transition}

In Section 3, we have analyzed equations that are within the pulled regime. We here analyze equations that are at the pushed/pulled transition. Leading edges of fronts within the pulled regime have the form $\Phi^{*}=(\alpha \xi+\beta) \mathrm{e}^{-\xi} \propto \xi \mathrm{e}^{-\xi}$ $(\xi \gg 1)$, cf. (2.17). Leading edges of fronts within the pushed regime are given by $\Phi^{\dagger} \propto \mathrm{e}^{-\lambda_{+}\left(v^{\dagger}\right) \xi}$, cf. (2.23). Leading edges of fronts at the pushed/pulled transition accordingly behave as

$$
\Phi^{*}=\beta \mathrm{e}^{-\xi} \text { for } \xi \gg 1, \quad \lambda_{+}\left(v^{*}\right)=\lambda^{*}=1 .
$$

For our example nonlinearity (1.10), fronts are within the pulled regime for $\epsilon>(n+1) / n^{2}$ and at the pushed/pulled transition for $\epsilon=(n+1) / n^{2}$. The analysis below can again be extended to more general equations along the lines of Section 5 . We will come back to this at the end of this appendix.

At the pushed/pulled transition, the spectrum of linear perturbations is still gapless, and convergence therefore is algebraic. On the other hand, the form of the leading edge played a crucial role in determining the velocity corrections $\dot{X}$. Compare our qualitative discussion in Section 3.1.1. The leading edge behavior (G.1) immediately lets us expect, that now $v(t)=2-1 /(2 t)+\cdots$, in contrast to (3.5) and (3.66) for fronts within the pulled regime, and in agreement with (2.46) for the spreading of perturbations under the linearized equation. Intuitively, we can argue that the slower convergence of fronts within the pulled regime is due to the leading edge having to pull the interior part of the front along. This also makes the leading edge flatter. The quicker convergence of fronts at the pushed/pulled transition and in the linearized equation then resembles the fact that the leading edge and the interior part of the front "impose the same speed".

Let us now do the explicit convergence analysis for fronts developing from initial conditions steeper than $\mathrm{e}^{-x}$ for $x \gg 1$ and approaching (G.1) for large times. The analysis of the interior is identical with Section 3.2, where constants $c_{n / 2}$ are yet undetermined. When expanding the interior shape towards the leading edge as in Section 3.3, the inhomogeneities created by $\Phi^{*}$ (G.1) are different, because now $\alpha=0$. The differential equations for the $\psi_{n / 2}$ result from (3.34) with $\alpha=0, \gamma=\beta$ and start with

$$
\partial_{\xi}^{2} \psi_{1}=c_{1} \beta, \quad \partial_{\xi}^{2} \psi_{3 / 2}=c_{3 / 2} \beta, \quad \partial_{\xi}^{2} \psi_{2}=\left[-1+c_{1}\left(1-\partial_{\xi}\right)\right] \psi_{1}+c_{2} \beta+\mathrm{o}\left(\mathrm{e}^{-\xi_{X}}\right), \quad \text { etc. }
$$

Integrating and resumming, we now find for $\xi \gg 1$

$$
\begin{aligned}
\psi= & \beta+\frac{c_{1} \beta \xi_{X}^{2}}{2 ! t}+\frac{c_{1} \delta \xi_{X}}{t}+\mathrm{O}\left(\frac{1}{t}\right)+\frac{c_{3 / 2} \beta \xi_{X}^{2}}{2 ! t^{3 / 2}}+\mathrm{O}\left(\frac{\xi_{X}}{t^{3 / 2}}\right)+\frac{c_{1}\left(c_{1}-1\right) \beta \xi_{X}^{4}}{4 ! t^{2}} \\
& +\frac{c_{1}\left(c_{1} \delta-\delta-c_{1} \beta\right) \xi_{X}^{3}}{3 ! t^{2}}+\mathrm{O}\left(\frac{\xi_{X}^{2}}{t^{2}}\right)+\frac{c_{3 / 2}\left(2 c_{1}-\frac{3}{2}\right) \beta \xi_{X}^{4}}{4 ! t^{5 / 2}}+\mathrm{O}\left(\frac{\xi_{X}^{3}}{t^{5 / 2}}\right)+\cdots+\cdots
\end{aligned}
$$

Here $\delta$ is an unknown integration constant fixed by condition (3.9). We will see below, that it is not involved in fixing the velocity, just as also the subleading $\beta$ for the leading edge (3.32) within the pulled regime is not involved in fixing the velocity, cf. the calculation till (3.65).

Again for $\xi_{X} \gg \sqrt{t}$, we have to reorder the expansion in powers of $\sqrt{z}=\left(\xi_{X}^{2} /(4 t)\right)^{1 / 2}$ and $1 / \sqrt{t}$, and find

$$
\begin{aligned}
\psi= & \beta\left(1+\frac{c_{1}(4 z)}{2 !}+\frac{c_{1}\left(c_{1}-1\right)(4 z)^{2}}{4 !}+\mathrm{O}\left(z^{3}\right)\right) \\
& +\frac{1}{\sqrt{t}}\left(c_{1} \delta(4 z)^{1 / 2}+\frac{c_{3 / 2} \beta(4 z)}{2 !}+\frac{c_{1}\left(c_{1} \delta-\delta-c_{1} \beta\right)(4 z)^{3 / 2}}{3 !}+\frac{c_{3 / 2}\left(2 c_{1}-\frac{3}{2}\right) \beta(4 z)^{2}}{4 !}\right)+\mathrm{O}\left(\frac{1}{t}\right) .
\end{aligned}
$$


The structure of the expansion is the same as in (3.42), except that now the leading order term is of order $t^{0}$ :

$$
G(z, t)=\mathrm{e}^{z} \psi=g_{0}(z)+\frac{g_{1 / 2}(z)}{\sqrt{t}}+\cdots
$$

The equations of motion for the leading and subleading term are derived from (3.43)-(3.45) through putting $g_{-1 / 2}=0$. For $g_{0}$, we find now the homogenous equation

$$
\left[z \partial_{z}^{2}+\left(\frac{1}{2}-z\right) \partial_{z}-\frac{1}{2}-c_{1}\right] g_{0}=0 .
$$

Just like (3.43) was solved by (3.52), we now solve (G.6) with

$$
c_{1}=-\frac{1}{2}, \quad g_{0}(z)=\beta .
$$

The equation for $g_{1 / 2}$ is now, cf. (3.45) and (G.7),

$$
\left[z \partial_{z}^{2}+\left(\frac{1}{2}-z\right) \partial_{z}+\frac{1}{2}\right] g_{1 / 2}=\beta\left[c_{3 / 2}-\frac{1}{2} \sqrt{z}\right] .
$$

Again a special solution of the inhomogeneous equation can be found, and the general solution contains the constants of integration $k_{1 / 2}$ and $l_{1 / 2}$ :

$$
\begin{aligned}
& g_{1 / 2}=\beta\left[2 c_{3 / 2}-\frac{\sqrt{z}}{2} \sum_{n=1}^{\infty} \frac{(1)_{n-1} z^{n}}{\left(\frac{3}{2}\right)_{n} n !}\right]+k_{1 / 2} M\left(-\frac{1}{2}, \frac{1}{2}, z\right)+l_{1 / 2} \sqrt{z} \\
& \stackrel{z}{=} 2 \beta c_{3 / 2}+k_{1 / 2}+l_{1 / 2} \sqrt{z}+\mathrm{O}(z) \\
& \underset{z \rightarrow \infty}{\sim} \frac{-\beta \sqrt{\pi}}{4 z} \mathrm{e}^{z}-\frac{k_{1 / 2}}{2 z} \mathrm{e}^{z} .
\end{aligned}
$$

Comparing (G.10) to the order $1 / \sqrt{t}$ in (G.4) and imposing proper convergence of (G.11) for $z \rightarrow \infty$, we find

$$
2 \beta c_{3 / 2}+k_{1 / 2}=0, \quad l_{1 / 2}=-\delta, \quad \beta \sqrt{\pi}+2 k_{1 / 2}=0 .
$$

With these constants, the velocity correction $c_{3 / 2}$ is

$$
c_{3 / 2}=\frac{1}{4} \sqrt{\pi},
$$

and for $g_{1 / 2}$ we find

$$
g_{1 / 2}=\frac{\beta \sqrt{\pi}}{2}\left[1-M\left(-\frac{1}{2}, \frac{1}{2}, z\right)-\left(\frac{z}{\pi}\right)^{1 / 2} \sum_{n=1}^{\infty} \frac{(1)_{n-1} z^{n}}{\left(\frac{3}{2}\right)_{n} n !}\right]-\delta \sqrt{z} .
$$

In summary, we find for the convergence to a front at the pushed/pulled transition, whose leading edge accordingly takes the form (G.1), that the velocity correction is given by

$$
\dot{X}=-\frac{1}{2 t}\left(1-\frac{1}{2}\left(\frac{\pi}{t}\right)^{1 / 2}\right)+\mathrm{O}\left(\frac{1}{t^{2}}\right) \text {. }
$$

In the interior, i.e., for $\xi_{X} \ll \sqrt{t}$, the front is given by (3.31) just like a front within the pulled regime. In the leading edge, where $\xi_{X} \gg \sqrt{t}$, the front is given by

$$
\phi\left(\xi_{X}, t\right)=\exp \left\{\xi_{X}-\xi_{X}^{2} /(4 t)\right\} G\left(\frac{\xi_{X}^{2}}{4 t}, t\right)
$$


where

$$
G(z, t)=\beta+\frac{g_{1 / 2}(z)}{\sqrt{t}}+\mathrm{O}\left(\frac{1}{t}\right)
$$

The extension along the lines of Section 5 to more general equations is straightforward. The general expression for $\dot{X}(t)$ is

$$
\dot{X}=-\frac{1}{2 \lambda^{*} t}\left(1-\frac{1}{2 \lambda^{*}}\left(\frac{\pi}{D t}\right)^{1 / 2}\right)+\mathrm{O}\left(\frac{1}{t^{2}}\right)
$$

but the subleading function $g_{1 / 2}(z)$ will depend on the additional terms in the expansion, just like the subleading $g_{0}(z)$ in Section 5.3.

Appendix H. Multiplicity of fronts and linear eigenmodes for reflection symmetric equations of first order in time

The generical multiplicity of uniformly translating fronts $\Phi_{v}$ can be determined by counting arguments analogous to those performed in Section 2.2. Uniformly translating solutions $\Phi_{v}(\xi)$ of (5.1) can be understood as a heteroclinic orbit in $N$-dimensional phase space between fixed points characterized by $\Phi_{v}=1$ at $\xi \rightarrow-\infty$ and $\Phi_{v}=0$ at $\xi \rightarrow \infty$. For a linear perturbation $\delta=1-\Phi_{v}$ about the fixed point $\phi=1$ from (5.2), we get the equation

$$
\mathcal{L}_{v}(-\infty) \delta+\mathrm{O}\left(\delta^{2}\right)=0
$$

which is a linear ODE with constant coefficients with the linear operator $\mathcal{L}$ being defined in (5.3). The same is true for a linear perturbation $\Phi_{v}=0+\delta$ of the fixed point $\phi=0$, which solves

$$
\mathcal{L}_{v}(\infty) \delta+\mathrm{O}\left(\delta^{2}\right)=0
$$

In linear order of $\delta$, each of these equations has $N$ solutions $\mathrm{e}^{-\lambda_{n}(v) \xi}, n=1, \ldots, N$.

Let us restrict the analysis to real equations which are isotropic in space, i.e., where (5.41) is invariant under $x \rightarrow-x$. Such equations are even in $\partial_{x}$, so $N$ needs to be even. According to arguments presented in Appendix A of [65], (H.1) and (H.2) for $v>0$ will have $\frac{1}{2} N+1$ eigenvalues $\lambda_{n}$ with positive real part and $\frac{1}{2} N-1$ ones with a negative real part, if the state, about which we linearize, is linearly unstable against a range of Fourier modes. If it is stable, we will have $\frac{1}{2} N$ eigenvalues with positive real part and $\frac{1}{2} N$ ones with a negative real part. We assume $\phi=1$ to be stable, so at $\xi \rightarrow-\infty$ there are $\frac{1}{2} N$ directions in phase space with negative real part of $\lambda$, that need to be excluded. If $\phi=0$ is unstable, we have only $\frac{1}{2} N-1$ bad eigendirections at $\xi \rightarrow \infty$. We then generically have a front connecting these fixed points for arbitrary values of $v$. If, however, the state $\phi=0$ is metastable, there are $\frac{1}{2} N$ bad eigendirections at $\xi \rightarrow \infty$. Then also $v$ needs to be tuned to find a solution. So for fronts propagating into unstable states, we generically have a front solution $\Phi_{v}$ for a continuum of velocities, while for fronts into metastable states, there are solutions $\Phi_{v}$ only for discrete values of $v$, in generalization of the arguments from Section 2.2.

The multiplicity of linear perturbations is determined along the same lines. We again decompose the linear perturbations $\eta(5.30)$ into $\eta(\xi, t)=\eta_{\sigma}(\xi) \mathrm{e}^{-\sigma t}$ by separation of variables. The $\eta_{\sigma}$ then solve the ODE

$$
\left[\mathcal{L}_{v}(\xi)+\sigma\right] \eta_{\sigma}(\xi)=0 .
$$

For counting the generic multiplicity of solutions, we need to linearize the equations about $\xi \rightarrow \pm \infty$, which amounts to a problem equivalent to (H.1) and (H.2), except for a shift of the constant contribution of $\mathcal{L}_{v}(\xi)$ by $\sigma$. For fronts propagating into unstable states, we in general expect a continuous spectrum $\sigma$ of linear perturbations at least in some finite interval of $\sigma$, in generalization of Section 2.3. 
Appendix I. Strongly heteroclinic orbits and change of stability at $v^{\dagger}$

According to the counting argument from Appendix $\mathrm{H}$, the front $\Phi^{*}(\xi)$ propagating uniformly with velocity $v^{*}$ does exist. The question is now, whether it is stable and whether it will be approached by steep initial conditions. In particular, we want to analyze initial conditions $\phi(x, 0)$, that are steeper than $\mathrm{e}^{-\lambda^{*} x}$ in the leading edge.

This amounts to the question, whether in the spectral decomposition $\eta_{\sigma}(\mathrm{H} .3)$ of a generic $\phi(x, 0)-\Phi^{*}(x)$, there are destablizing modes with $\sigma<0$. As in Section 2.3, the contributing modes in general will all decay at least as quick as $\Phi^{*}$ in the leading edge. The leading edge properties of the $\eta_{\sigma}$ in general will depend smoothly on $\sigma$, just as in (D.14), so generically $\Phi^{*}$ will still be stable against all perturbations, that in the leading edge decay quicker than $\Phi^{*}$.

An exemption is again the generalization of $A_{\sigma}=0$ from (D.9). For an equation of order $N$ with $\phi=0$ unstable, there are $\frac{1}{2} N+1$ exponents $\Lambda_{n}(\sigma, v)>0$. The leading edge will be a superposition of all the exponentials

$$
\eta_{\sigma}(\xi)=\sum_{n=1}^{\frac{1}{2} N+1} A_{\sigma}^{(n)} \mathrm{e}^{-\Lambda_{n} \xi} \quad \text { as } \quad \xi \gg 1
$$

The condition $A_{\sigma}^{(1)}=0$, where $\Lambda_{1}$ is the smallest one of the positive $\Lambda_{n}$, fixes a discrete set (which can be either empty or not empty) of negative $\sigma$ 's whose eigenfunctions $\eta_{\sigma}$ have a steepness in the leading edge larger than $\Phi^{*}$. The stability of the pulled front $\Phi^{*}$ thus again depends on the "strongly heteroclinic" perturbations.

If there are strongly heteroclinic perturbations, that destabilize the pulled front propagating with velocity $v^{*}$, then there will be a steeper and quicker front $\Phi^{\dagger}$, which can be constructed as a strongly heteroclinic orbit of (5.41). The zero mode $\partial_{\xi} \Phi^{\dagger}$ then again is a strongly heteroclinic perturbation, and as discussed in Appendix D on the stability of front solutions, we can conclude that the quickest of all strongly heteroclinic orbits cannot be destabilized, so it will attract all sufficiently steep initial conditions.

We conclude, that Table 4 generalizes to higher order equations, which form uniformly translating fronts, if we only appropriately adjust the explicit definitions of the velocities $v$ and steepnesses $\lambda$.

\section{Appendix J. Relation between the generalized diffusion constants $D_{n}$ and the dispersion relation}

If we use the expansion (5.6) for the dispersion relation $\omega(k)$, we get

$$
\begin{aligned}
\mathcal{D} & =\mathrm{e}^{\lambda^{*} \xi}\left(\sum_{m=0}^{N} a_{m} \partial_{\xi}^{m}-v^{*} \partial_{\xi}\right) \mathrm{e}^{-\lambda^{*} \xi}=\sum_{m=0}^{N} a_{m}\left(\partial_{\xi}-\lambda^{*}\right)^{m}-v^{*}\left(\partial_{\xi}-\lambda^{*}\right) \\
& =\sum_{m=0}^{N} \sum_{n=0}^{m} a_{m} \frac{m !}{n !(m-n) !}\left(-\lambda^{*}\right)^{(m-n)} \partial_{\xi}^{n}-v^{*}\left(\partial_{\xi}-\lambda^{*}\right) \\
& =\sum_{n=0}^{N}\left(\frac{\partial^{n}}{\partial\left(-\lambda^{*}\right)^{n}} \sum_{m=n}^{N} a_{m}\left(-\lambda^{*}\right)^{m}\right) \frac{1}{n !} \partial_{\xi}^{n}-v^{*}\left(\partial_{\xi}-\lambda^{*}\right) .
\end{aligned}
$$

This immediately yields the expansion (5.27) with the identification (5.28). 


\section{Appendix K. Edge analysis of uniformly translating pulled fronts with $M=1$}

We analyze the leading edge representation (5.37) for a uniformly translating front whose equation of motion (5.41) is of arbitrary order $N$ in space and of first order in time $M=1$ :

$$
\partial_{\tau} \psi=\left(\partial_{\zeta}^{2}+\sum_{n=3}^{N} d_{n} \partial_{\zeta}^{n}\right) \psi+\dot{Y}\left(\partial_{\zeta}-1\right) \psi
$$

We generalize the leading edge analysis from Sections 3.3 and 3.4.

With the notions and ansatz

$$
\begin{aligned}
& \mathcal{D}=\partial_{\zeta}^{2}+\sum_{n=3}^{N} d_{n} \partial_{\zeta}^{n}, \quad \dot{Y}=\sum_{n=2}^{\infty} \frac{C_{n / 2}}{\tau^{n / 2}}, \\
& \psi\left(\zeta_{Y}, \tau\right)=\alpha \zeta Y+\beta+\frac{\psi_{1 / 2}}{\tau^{1 / 2}}+\frac{\psi_{1}}{\tau}+\frac{\psi_{3 / 2}}{\tau^{3 / 2}}+\cdots,
\end{aligned}
$$

the expansion of the interior in the region of $\zeta Y \gg 1$ at the crossover towards the leading edge reads

$$
\mathcal{D} \psi_{1 / 2}=0, \quad \mathcal{D} \psi_{1}=C_{1}\left(\alpha \zeta_{Y}+\gamma\right), \quad \gamma=\beta-\alpha, \quad \mathcal{D} \psi_{3 / 2}=C_{3 / 2}\left(\alpha \zeta_{Y}+\gamma\right), \ldots
$$

in generalization of (3.34). These equations can be integrated explicitly. The result can be written in leading edge variables $z=\zeta_{Y}^{2} /(4 \tau)$ as

$$
\psi=\sqrt{\tau} \alpha\left((4 z)^{1 / 2}+\frac{C_{1}(4 z)^{3 / 2}}{3 !}+\cdots\right)+\tau^{0}\left(\beta+\frac{C_{1}\left(\beta-\alpha\left(1+d_{3}\right)\right)(4 z)}{2 !}+\cdots\right)+\mathrm{O}\left(\frac{1}{\sqrt{\tau}}\right) .
$$

This generalizes the results of Section 3.3 and supplies us with the small $z$ expansion of the leading edge function

$$
\psi\left(\zeta_{4}, \tau\right)=\mathrm{e}^{-z} G(z, \tau), \quad z=\frac{\zeta_{4}^{2}}{4 \tau}
$$

$G$ solves (compare Eq. (3.41))

$$
\begin{aligned}
& {\left[z \partial_{z}^{2}+\left(\frac{1}{2}-z\right) \partial_{z}-\frac{1}{2}-\tau \partial_{\tau}-C_{1}\right] G} \\
& \quad=\frac{1}{\sqrt{\tau}}\left[C_{3 / 2}+C_{1} \sqrt{z}\left(1-\partial_{z}\right)\right] G-\frac{d_{3} \sqrt{z}}{\sqrt{\tau}}\left[\frac{3}{2}\left(\partial_{z}-1\right)^{2}+z\left(\partial_{z}-1\right)^{3}\right] G+\mathrm{O}\left(\frac{1}{\tau}\right),
\end{aligned}
$$

where we wrote all operators of order $\tau^{0}$ on the LHS of the equation and the operators of order $\tau^{-1 / 2}$ on the RHS. With the ansatz

$$
G(z, \tau)=\sqrt{\tau} g_{-1 / 2}(z)+g_{0}(z)+\frac{g_{1 / 2}(z)}{\sqrt{\tau}}+\cdots
$$

as in (3.42), we find that $g_{-1 / 2}(z)$ solves again (3.43), so we copy from Section 3.4 , that

$$
C_{1}=-\frac{3}{2}, \quad g_{-1 / 2}(z)=2 \alpha \sqrt{z} .
$$

For $g_{0}(z)$ we then find instead of (3.55):

$$
\left[z \partial_{z}^{2}+\left(\frac{1}{2}-z\right) \partial_{z}+1\right] g_{0}=2 \alpha\left[\frac{3}{4}\left(1+d_{3}\right)+c_{3 / 2} \sqrt{z}-\frac{3}{2} z+d_{3}\left(z^{2}-3 z\right)\right] .
$$


A special solution of the inhomogeneous equation is now instead of (3.58):

$$
g_{0}^{\mathrm{sp}}(z)=2 \alpha\left(\frac{3}{4}\left(1+d_{3}\right)+2 c_{3 / 2} \sqrt{z}-\frac{3}{4} F_{2}(z)-d_{3} z^{2}\right),
$$

with $F_{2}(z)$ from (3.56). The general solution is

$$
\begin{aligned}
g_{0}(z) & =g_{0}^{\mathrm{sp}}(z)+k_{0}(1-2 z)+l_{0} \sqrt{z} M\left(-\frac{1}{2}, \frac{3}{2}, z\right) \\
& \stackrel{z}{=}\left(\frac{3}{2} \alpha\left(1+d_{3}\right)+k_{0}\right)+\left(4 \alpha c_{3 / 2}+l_{0}\right) \sqrt{z}+\mathrm{O}(z) \\
& z \rightarrow \infty-\left(\frac{3}{2} \alpha \sqrt{\pi}+\frac{1}{4} l_{0}\right) z^{-3 / 2} \mathrm{e}^{z},
\end{aligned}
$$

Note, that $d_{3} \neq 0$ does not cause any divergences at $z \rightarrow \infty$. It only shifts the constant contribution at $z \rightarrow 0$.

Suppressing the divergence at $z \rightarrow \infty$ in (K.12), and comparing its small $z$ expansion to (K.5) yields again

$$
\begin{aligned}
& C_{3 / 2}=\frac{3}{2} \sqrt{\pi}, \\
& g_{0}(z)=\beta(1-2 z)+3 \alpha\left(1+d_{3}\right) z-2 \alpha d_{3} z^{2}-\frac{3}{2} \alpha F_{2}(z)+6 \alpha \sqrt{\pi z}\left(1-M\left(-\frac{1}{2}, \frac{3}{2}, z\right)\right) .
\end{aligned}
$$

\section{Appendix L. Leading edge projections for coupled equations: an example}

As a simple illustration of the various questions related to the projection discussed in Section 5.5.3, we consider two coupled F-KPP equations,

$$
\partial_{t} \phi_{1}=\partial_{x}^{2} \phi_{1}+\phi_{1}-\phi_{1}^{3}, \quad \partial_{t} \phi_{2}=D \partial_{x}^{2} \phi_{2}+\phi_{2}-\phi_{2}^{3}+K \phi_{1} .
$$

The dynamics of this set of equations for fronts propagating into the state $\phi_{1}=\phi_{2}=0$ with steep initial conditions, is of course immediately obvious: when $K=0$, the two equations are uncoupled, and fronts in the first equation propagate with speed $v_{1}^{*}=2$, while those in the second equation propagate with speed $v_{2}^{*}=2 D^{1 / 2}$. The dynamics of $\phi_{1}$ is always independent of that of $\phi_{2}$, even for $K \neq 0$, so for $K>0$ and $D<1$, the dynamics of the coupled equations amounts to a normal F-KPP $\phi_{1}$ front, with relaxation given by our usual expressions. This front entrains a front with speed $v=v_{1}^{*}=2$ in $\phi_{2}$. For $D>1$, the $\phi_{1}$ and $\phi_{2}$ fronts keep on propagating with different speeds. We consider the case $D<1$ and make a leading edge transformation $\phi_{1}=\mathrm{e}^{-\xi} \psi_{1}, \phi_{2}=\mathrm{e}^{-\xi} \psi_{2}$ (where $\lambda^{*}=1$ ) to the frame moving with velocity $\xi=x-v_{1}^{*} t$. The linearized equations then become

$$
\partial_{t} \psi_{1}=\partial_{\xi}^{2} \psi_{1}, \quad \partial_{t} \psi_{2}=D \partial_{\xi}^{2} \psi_{2}+2(D-1) \partial_{\xi} \psi_{2}+(D-1) \psi_{2}+K \psi_{1} .
$$

The matrix $\underline{S}^{*}(q, \Omega)$ of the linearized equations is in this case

$$
\underline{S}^{*}(q, \Omega)=\left(\begin{array}{cc}
\mathrm{i} \Omega-q^{2} & 0 \\
K & \mathrm{i} \Omega-q^{2}+J(q)
\end{array}\right),
$$

where $J(q)=(D-1)\left(1+2 \mathrm{i} q-q^{2}\right)$. Since the element $S_{12}^{*}(q, \Omega)=0$, the eigenvalues $u_{1}^{*}$ and $u_{2}^{*}$ are simply the diagonal element of $\underline{S}^{*}(q, \Omega), u_{1}^{*}(q, \Omega)=\mathrm{i} \Omega-q^{2}$ and $u_{2}^{*}(q, \Omega)=\mathrm{i} \Omega-q^{2}+J(q)$. However, the eigenvectors are not both along the $\psi_{1}$ and $\psi_{2}$ axis. Indeed, we have in the notation of Section 5.5

$$
\begin{aligned}
& \underline{U}_{1}^{*}(q)=\left(\begin{array}{c}
1 \\
-K / J(q)
\end{array}\right), \quad \underline{U}_{1}^{* \dagger}=(1,0), \\
& \underline{U}_{2}^{*}=\left(\begin{array}{l}
0 \\
1
\end{array}\right), \quad \underline{U}_{2}^{* \dagger}(q)=(K / J(q), 1),
\end{aligned}
$$


The appropriate saddle point is $\Omega=q=0$, and since $J(0)=(D-1)$, we have

$$
\underline{U}_{1}^{*}(0)=\left(\begin{array}{c}
1 \\
-K /(D-1)
\end{array}\right) .
$$

The fact that the second component is non-zero just expresses the fact that the variable $\psi_{2}$ is entrained by the leading edge in $\psi_{1}$. We can now illustrate our assertion that different choices of projection lead to different dynamical equations for the projected leading edge variable $\psi^{\mathrm{p}}$, but that the universal results from Table 2 are independent of the particular choice of projection. Clearly, one obvious intuitively appealing choice is to take $\psi^{\mathrm{p}}=\psi_{1}$, since the $\psi_{1}$ dynamics is independent of that of $\psi_{2}$. In this case, the dynamical equation for $\psi^{\mathrm{P}}$ is nothing but the single F-KPP equation, and all the results for this equation carry over in detail. Likewise, the choice $\psi^{\mathrm{P}}=\pi_{1}(q, \Omega)(5.86)$ leads to the linearized F-KPP equation for $\psi^{\mathrm{p}}$ since $u_{1}^{*}(q, \Omega(q))=0$ gives the dispersion relation of the F-KPP equation. However, this choice is more formal than practical, since the direction in the vector space $\left(\psi_{1}, \psi_{2}\right)$ is not fixed, but depends on the variable $q$ which influences the dynamics. A more practical choice for the coupled variables would be to take $\psi^{\mathrm{p}}$ as the component along $\underline{U}_{1}^{*}(0)$, as this corresponds to a fixed ratio of $\psi_{1}$ and $\psi_{2}$. Since $\underline{U}_{2}^{* \dagger} \cdot \underline{U}_{1}^{*}=K / J(q)-K / J(0)=-2 K \mathrm{i} q /(D-1)+\mathrm{O}\left(q^{2}\right)$, the projected equation in this case picks up a third order derivative term $D_{3} \partial_{\xi}^{3} \psi^{\mathrm{p}}$, among other ones.

Thus, we observe in this particular example, that indeed the universal results from Table 2 on velocity and shape relaxation are independent of the choice of projection, while the subleading contribution $g_{0}(z)$ in the leading edge is universal in the sense, that it is independent of the precise initial conditions, but it does depend on the direction of projection.

\section{Appendix M. Pinch point versus saddle point analysis}

In this appendix, we briefly discuss the major differences and similarities between the saddle point and the pinch point approach for evaluating the integral

$$
\mathcal{I}_{m}=\int_{\mathrm{i} \gamma-\infty}^{\mathrm{i} \gamma+\infty} \frac{d \omega}{2 \pi} \int_{-\infty}^{\infty} \frac{\mathrm{d} k}{2 \pi} \exp \{\mathrm{i} k \xi-\mathrm{i}(\omega-v k) t\} \frac{\hat{\underline{M}}_{m}(k, \omega)}{u_{m}(k, \omega)}
$$

from Eq. (5.79) on a given branch $m$. Here $\gamma>0$ needs to be large enough, that the integrand is analytic along and above the path of $\omega$ integration in the complex $\omega$ plane. We introduced the abbreviation $\underline{\underline{M}}_{m}(k, \omega)=\underline{\hat{U}}_{m}(k, \omega) \times$ $\hat{\underline{U}}_{m}^{\dagger}(k, \omega)$. In the moving frame $\xi$, it is obviously convenient to transform to the variable $\Omega=\omega-v k$, and to introduce

$$
u_{m}^{v}(k, \Omega)=u_{m}(k, \Omega+v k)=u_{m}(k, \omega), \quad \Omega=\omega-v k
$$

The characteristic equation

$$
u_{m}\left(k, \omega_{m}(k)\right)=0 \Leftrightarrow u_{m}^{v}\left(k, \Omega_{m}(k)\right)=0
$$

defines the dispersion relation $\omega_{m}(k)$ or $\Omega_{m}(k)$. The integrals are now of the form

$$
\mathcal{I}_{m}=\int_{\mathrm{i} \gamma-\infty}^{\mathrm{i} \gamma+\infty} \frac{\mathrm{d} \Omega}{2 \pi} \int_{-\infty}^{\infty} \frac{\mathrm{d} k}{2 \pi} \exp \{\mathrm{i} k \xi-\mathrm{i} \Omega t\} \frac{\hat{\underline{M}}_{m}(k, \Omega+v k)}{u_{m}^{v}(k, \Omega)} .
$$


The "saddle point" type approach, that we follow in Sections 5.3,5.4 and 5.5 of this paper, is based on first evaluating the $\Omega$ integral by closing the $\Omega$ contour in the lower half plane for $t>0$ around the simple pole $\propto\left(\Omega-\Omega_{m}(k)\right)$. The integral then yields

$$
\mathcal{I}_{m}=\int_{-\infty}^{\infty} \frac{\mathrm{d} k}{2 \pi} \exp \left\{\mathrm{i} k \xi-\mathrm{i} \Omega_{m}(k) t\right\} \frac{\hat{\underline{M}}_{m}\left(k, \Omega_{m}(k)+v k\right)}{\mathrm{i} \partial \Omega_{m} u_{m}^{v}\left(k, \Omega_{m}(k)\right)},
$$

where $\gamma$ needs to be larger than $\max _{k}$ real $\left(\operatorname{Im} \Omega_{m}(k)\right)$. From here on, the saddle point analysis proceeds essentially as in Section 5.4: the $k$-contour is deformed continuously such that it passes through a saddle point of $\Omega_{m}(k)$ that allows for a steepest descent evaluation of the $k$-integral. A saddle point is a double root in $k$ of $u_{m}^{v}(k, \Omega)$, so that

$$
\begin{aligned}
& \left.u_{m}^{v}(k, \Omega)\right|_{\mathrm{sp}}=0 \quad \Leftrightarrow \quad \omega_{\mathrm{sp}}=\omega_{m}\left(k_{\mathrm{sp}}\right) \quad \Leftrightarrow \quad \Omega_{\mathrm{sp}}=\omega_{m}\left(k_{\mathrm{sp}}\right)-v k_{\mathrm{sp}}, \\
& \left.\partial_{k} u_{m}^{v}(k, \Omega)\right|_{\mathrm{sp}}=\left.0 \quad \Leftrightarrow \quad\left(\partial_{k}+v \partial_{\omega}\right) u_{m}(k, \omega)\right|_{\mathrm{sp}}=0 \quad \Leftrightarrow \quad v=-\frac{\left.\partial_{k} u_{m}(k, \omega)\right|_{\mathrm{sp}}}{\left.\partial_{\omega} u_{m}(k, \omega)\right|_{\mathrm{sp}}} .
\end{aligned}
$$

By expanding about such a saddle point, we get for large $t$ to leading order

$$
\mathcal{I}_{m}=\left.\frac{\hat{\underline{M}}_{m}(k, \omega)}{\mathrm{i} \partial_{\omega} u_{m}(k, \omega)}\right|_{\mathrm{sp}} \exp \left\{\mathrm{i} k_{\mathrm{sp}} \xi-\mathrm{i} \Omega_{\mathrm{sp}} t\right\} \int_{q} \exp \left\{\mathrm{i} q \xi-D_{\mathrm{sp}} q^{2} t\right\}+\cdots,
$$

with the diffusion constant

$$
D_{\mathrm{sp}}=\frac{-\left.\mathrm{i}\left(\partial_{k}+v \partial_{\omega}\right)^{2} u_{m}\right|_{\mathrm{sp}}}{\left.2 \partial_{\omega} u_{m}\right|_{\mathrm{sp}}}=\frac{-\left.\mathrm{i} \partial_{k}^{2} u_{m}^{v}\right|_{\mathrm{sp}}}{\left.2 \partial_{\omega} u_{m}^{v}\right|_{\mathrm{sp}}} .
$$

The remaining integral over real $q=k-k_{\mathrm{sp}}$ is a simple Gaussian integral of the form discussed previously in Section 5.3.1. As before, we are in the comoving frame, if

$$
\operatorname{Im} \Omega_{\mathrm{sp}}=0 \Leftrightarrow v=\frac{\operatorname{Im} \omega_{m}\left(k_{\mathrm{sp}}\right)}{\operatorname{Im} k_{\mathrm{sp}}} .
$$

Differentiating the dispersion relation $u\left(k, \omega_{m}(k)\right)=0$ with respect to $k: \partial_{k} u\left(k, \omega_{m}(k)\right)=0$, and comparing to (M.7), we can immediately identify

$$
v=\left.\frac{\partial \omega_{m}(k)}{\partial k}\right|_{\mathrm{sp}}
$$

From $\partial_{k}^{2} u\left(k, \omega_{m}(k)\right)=0$ and (M.9), we get

$$
D=\left.\frac{\mathrm{i} \partial^{2} \omega_{m}(k)}{2 \partial k^{2}}\right|_{\mathrm{sp}} .
$$

Choosing in (5.79) the branch $m$ with the largest velocity $v_{\mathrm{sp}}=v^{*}$, Eq. (5.81) immediately results.

If the denominator of an integral like (M.4) contains a product of characteristic functions $\prod_{m=1}^{M} u_{m}^{v}(k, \Omega)$, then each factor $u_{m}^{v}(k, \Omega)$ will contribute with its pole and yield an integral as in (M.5), so that the total integral amounts to a sum of $M$ integrals of the form (M.5). Again the dominating contribution for $\xi$ fixed and $t \gg 1$ will be the one with the largest velocity $v_{\mathrm{sp}}$ through which the contour of $k$-integration can be deformed.

The pinch point analysis [56] is based on evaluating (M.1) by a different order of the integrations, i.e., by first closing the $k$-contour to get $k=k(\Omega)$ and then evaluating the remaining $\Omega$ integral. (For $\xi>0$, the $k$-contour must be closed in the upper half plane.) As discussed most clearly by Bers [56], this is done as follows. $\gamma$ in (M.4) has 
to be large enough to lie above the maxima of the dispersion relation $\Omega_{m}(k)$ for real $k$. When $\Omega$ varies along the integration path, the poles in the $k$-plane move. Now when $\gamma$ is lowered sufficiently, that it approaches the maximum of the line $\Omega_{m}(k)$ traced out by the real $k$ values, a pole in the $k$-plane will approach the real $k$-axis. When that happens, the $k$-contour can be continuously deformed to avoid this pole. This in turn allows one to lower the value of $\gamma$. This process can continue until two poles in the $k$-plane approach the $k$-contour from opposite sides, and "pinch off" the $k$-contour at a particular value of $\Omega^{*}$. Clearly, that point corresponds to a double root, since for that given value of $\Omega$ the two $k$ roots coincide. When the $k$-contour is closed, this point generates a branch-cut in the $\Omega$ plane, since near $\Omega^{*}$ we have $k-k^{*}= \pm\left(\left(\Omega-\Omega^{*}\right) / D\right)^{1 / 2}$. When the $\Omega$ contour is subsequently closed in the lower half $\Omega$ plane, these branch points then generate the usual leading asymptotic behavior ((5.14) and (5.15)).

In both approaches, there are conditions for a saddle or pinch point to be dynamically relevant; these arise from the global properties of the dispersion relation $\omega(k)$. In the saddle point approach, only saddle points that will dominate the $k$-integral along the deformed contour of integration, are relevant for the dynamics. Pictorially, a saddle point that obeys these conditions is located between "valleys" of $\operatorname{Im} \omega(k)$ in the direction of real $k$ that are not completely separated by "ridges" from the real $k$-axis. In this formulation, the condition $\operatorname{Re} D>0$ naturally comes out. If there is more than one such saddle point, the one with the highest velocity $v^{*}$ determines the asymptotic spreading velocity. In the pinch point formulation, the condition usually mentioned is that the poles in the $k$-plane "pinch off" the $k$-contour, while the condition $\operatorname{Re} D>0$ is usually not mentioned, but it is actually hidden in the formulation as well: it just expresses that the pinch point is associated with a point of the dispersion relation, where the growth rate is maximal. In fact, the examples discussed in [[56], pp. 466-467] for solutions of the saddle point equations which are no pinch points, are just cases where $\operatorname{Re} D<0$, i.e., solutions which are excluded by a saddle point formulation as well. In the pinch point formulation, the improper solutions of the saddle point equations correspond to solutions where two poles in the $k$-plane do not "pinch off" the deformed $k$-contour, but instead just merge by themselves.

\section{References}

[1] C. Mathis, M. Provansal, L. Boyer, J. Phys. Lett. 45 (1984) L483.

[2] G. Ahlers, D.S. Cannell, Phys. Rev. Lett. 50 (1983) 1583.

[3] K.L. Babcock, G. Ahlers, D.S. Cannell, Phys. Rev. Lett. 67 (1992) 3388.

[4] J. Fineberg, V. Steinberg, Phys. Rev. Lett. 58 (1987) 1332

[5] A. Tsameret, V. Steinberg, Phys. Rev. E 49 (1994) 1291.

[6] R.C. Ball, R.L.H. Essery, J. Phys. Cond. Mat. 2 (1990) 10303.

[7] L. Limat, P. Jenffer, B. Dagens, E. Touron, M. Fermigier, J.E. Wesfreid, Physica D 61 (1992) 166.

[8] J.S. Langer, H. Müller-Krumbhaar, Phys. Rev. A 27 (1983) 499.

[9] T.R. Powers, R.E. Goldstein, Phys. Rev. Lett. 78 (1997) 2555.

[10] R.J. Deissler, Phys. Lett. A 120 (1987) 334.

[11] R.J. Deissler, J. Stat. Phys. 54 (1989) 1459.

[12] K. Nozaki, N. Bekki, Phys. Rev. Lett. 51 (1983) 2171.

[13] E.K.H. Salje, J. Phys. Cond. Mat. 5 (1993) 4775.

[14] S.J. Di Bartolo, A.T. Dorsey, Phys. Rev. Lett. 77 (1996) 4442, and references therein.

[15] U. Ebert, W. van Saarloos, C. Caroli, Phys. Rev. Lett. 77 (1996) 4178.

[16] M. Niklas, M. Lücke, H. Müller-Krumbhaar, Phys. Rev. A 40 (1989) 493.

[17] R. van Zon, H. van Beijeren, Ch. Dellago, Phys. Rev. Lett. 80 (1998) 2035.

[18] C. Uhlig, J. Eggers, Z. Phys. B 103 (1997) 69.

[19] J.E. Maclennan, N.A. Cark, T. Carlsson, in: Solitons in Liquid Crystals, Springer, Berlin, 1991.

[20] W. van Saarloos, M. van Hecke, R. Hołyst, Phys. Rev. E 52 (1995) 1773.

[21] Y. Tu, M.C. Cross, Phys. Rev. Lett. 69 (1992) 2515.

[22] J. Krug, P. Meakin, Phys. Rev. A 43 (1991) 900.

[23] A. Torcini, P. Grassberger, A. Politi, J. Phys. A 28 (1995) 4533.

[24] M.L. van Hecke, E. de Wit, W. van Saarloos, Phys. Rev. Lett. 75 (1995) 3830.

[25] A. Couairon, J.M. Chomaz, Physica D 108 (1997) 236. 
[26] M. van Hecke, C. Storm, W. van Saarloos, Physica D 134 (1999) 1.

[27] D. Carpentier, P. Le Doussal, Phys. Rev. Lett. 81 (1998) 2558.

[28] M.C. Cross, P.C. Hohenberg, Rev. Mod. Phys. 65 (1992) 851

[29] Ya.B. Zeldovich, G.I. Barenblatt, V.B. Librovich, G.M. Makhviladze, The Mathematical Theory of Combustion and Explosions, Consultants Bureau. New York, 1985.

[30] P.C. Fife, Mathematical Aspects of Reacting and Diffusing Systems, Lecture Notes in Biomathematics, Vol. 28, Springer, Berlin, 1979.

[31] R.J. Field, M. Burger (Eds.), Oscillations and Traveling Waves in Chemical Systems, Wiley, New York, 1985.

[32] N.F. Britton, Reaction-Diffusion Equations and their Applications to Biology, Academic Press, New York, 1986.

[33] D. Horváth, V. Petrov, S.K. Scott, K. Showalter, J. Chem. Phys. 98 (1993) 6332.

[34] A. Malevanets, A. Careta, R. Kapral, Phys. Rev. E 52 (1995) 4724.

[35] D. Horváth, A. Tóth, J. Chem. Phys. 108 (1998) 1447.

[36] J.A. Murray, Mathematical Biology, Springer, Berlin, 1989.

[37] U. Ebert, W. van Saarloos, Phys. Rep. 337 (2000) 139-156.

[38] P.C. Fife, Dynamics of Internal Layers and Diffusive Interfaces, SIAM, Philadelphia, PA, 1988.

[39] A. Karma, W.-J. Rappel, , Phys. Rev. E 53 (1996) R3017.

[40] G.F. Mazenko, O.T. Valls, P. Ruggiero, Phys. Rev. B 40 (1989) 384.

[41] O.T. Valls, L.M. Lust, Phys. Rev. B 44 (1991) 4326.

[42] A. Lemarchand, A. Lesne, A. Perera, M. Moreau, M. Mareschal, Phys. Rev. E 48 (1993) 1568.

[43] H. Breuer, W. Huber, F. Petruccione, Physica D 73 (1994) 259.

[44] J. Riordan, C.R. Doering, D. ben-Avraham, Phys. Rev. Lett. 75 (1995) 565.

[45] J. Armero, J.M. Sancho, J. Casademunt, A.M. Lacasta, L. Ramirez-Piscina, F. Sagues, Phys. Rev. Lett. 76 (1996) 3045.

[46] G. Tripathy, W. van Saarloos, Fluctuation and relaxation properties of pulled fronts: a possible scenario for non-KPZ behavior cond-mat/000513, to appear in Phys. Rev. Lett.

[47] J. Armero, J. Casademunt, L. Ramrez-Piscina, J.M. Sancho, Phys. Rev. E 58 (1998) 5494.

[48] A. Rocco, U. Ebert, W. van Saarloos, Phys. Rev. E 62 (2000) R13.

[49] A. Kolmogoroff, I. Petrovsky, N. Piscounoff, in: P. Pelcé (Ed.), Dynamics of Curved Fronts, Academic Press, San Diego, 1988 [translated from: Bulletin de l'Université d'État à Moscou, Ser. Int., Section A, Vol. 1, 1937].

[50] R.A. Fisher, Ann. Eugenics 7 (1937) 355.

[51] D.G. Aronson, H.F. Weinberger, Adv. Math. 30 (1978) 33.

[52] P. Collet, J.P. Eckmann, Instabilities and Fronts in Extended Systems, Princeton University Press, Princeton, NJ, 1990.

[53] H.P. McKean, Commun. Pure Appl. Math. 28 (1975) 323.

[54] R.D. Benguria, M.C. Depassier, Phys. Rev. Lett. 77 (1996) 1171.

[55] T. Ogiwara, H. Matano, Discr. Cont. Dyn. Syst. 5 (1999) 1.

[56] A.N. Bers, in: M.N. Rosenbluth, R.Z. Sagdeev (Eds.), Handbook of Plasma Physics, North-Holland, Amsterdam, 1983.

[57] P. Huerre, in: J.E. Wesfreid, H.R. Brand, P. Manneville, G. Albinet, N. Boccara (Eds.), Propagation in Systems Far from Equilibrium, Springer, New York, 1988.

[58] L.D. Landau, E.M. Lifshitz, Course of Theoretical Physics, Vol. 10, Pergamon, New York, 1981.

[59] A.N. Stokes, Math. Biosci. 31 (1976) 307.

[60] G. Dee, J.S. Langer, Phys. Rev. Lett. 50 (1983) 383.

[61] E. Ben-Jacob, H.R. Brand, G. Dee, L. Kramer, J.S. Langer, Physica D 14 (1985) 348.

[62] G. Dee, J. Stat. Phys. 39 (1985) 705.

[63] W. van Saarloos, Phys. Rev. A 37 (1988) 211.

[64] G. Dee, W. van Saarloos, Phys. Rev. Lett. 60 (1988) 2641.

[65] W. van Saarloos, Phys. Rev. A 39 (1989) 6367.

[66] W. van Saarloos, P.C. Hohenberg, Physica D 56 (1992) 303

[67] J.A. Powell, A.C. Newell, C.K.R.T. Jones, Phys. Rev. A 44 (1991) 3636.

[68] G.C. Paquette, L.-Y. Chen, N. Goldenfeld, Y. Oono, Phys. Rev. Lett. 72 (1994) 76.

[69] G.C. Paquette, Y. Oono, Phys. Rev. E 49 (1994) 2368.

[70] E. Brunet, B. Derrida, Phys. Rev. E 56 (1997) 2597.

[71] J. Swift, P.C. Hohenberg, Phys. Rev. A 15 (1977) 319.

[72] W. Spruijt, Master's Thesis, Leiden University, Leiden, 1998, unpublished.

[73] C. Storm, W. Spruijt, U. Ebert, W. van Saarloos, Phys. Rev. E 61 (2000) R6063.

[74] M. Bramson, Mem. Am. Math. Soc. 44 (1983) 285.

[75] D.A. Kessler, Z. Ner, L.M. Sander, Phys. Rev. E 58 (1998) 107.

[76] R. Durrett, Stoch. Proc. Appl. 9 (1979) 117.

[77] Th. Gallay, G. Raugel, http://xxx.lanl.gov/patt-sol/9809007 and 9812007.

[78] T.R. Powers, D. Zhang, R.E. Goldstein, H.A. Stone, Phys. Fluids 10 (1998) 1052

[79] J.S. Langer, in: G. Grinstein, G. Mazenko (Eds.), Directions in Condensed Matter Physics, World Scientific, Singapore, 1986.

[80] P.W. Bates, P.C. Fife, R.A. Gardner, C.K.R.T. Jones, Physica D 104 (1997) 1. 
[81] J.D. Buckmaster, G.S.S. Lundford, Theory of Laminar Flames, Cambridge University Press, Cambridge, 1982.

[82] U. Ebert, W. van Saarloos, Phys. Rev. Lett. 80 (1998) 1650.

[83] W. van Saarloos, Phys. Rep. 301 (1998) 9.

[84] G.I. Barenblatt, Scaling, Self-similarity and Intermediate Asymptotics, Cambridge University Press, Cambridge, 1996.

[85] J. Canosa, IBM J. Res. Dev. 17 (1973) 307.

[86] A. Messiah, Quantum Mechanics, North-Holland, Amsterdam, 1974.

[87] D.A. Larson, SIAM J. Appl. Math. 34 (1978) 93.

[88] D.H. Sattinger, J. Diff. Eqn. 25 (1977) 130.

[89] W.I. Newman, J. Theor. Biol. 104 (1983) 473.

[90] K. Kirchgässner, J. Diff. Eqn. 96 (1992) 256.

[91] J.-P. Eckmann, C.E. Wayne, Commun. Math. Phys. 161 (1994) 323.

[92] T. Kapitula, J. Diff. Eqns. 112 (1994) 179.

[93] V.I. Arnold, Ordinary Differential Equations, MIT Press, Cambridge, MA, 1978.

[94] J. Guckenheimer, P. Holmes, Nonlinear Oscillations, Dynamical Systems, and Bifurcations of Vector Fields, Springer, New York, 1983.

[95] J.D. Gunton, M. San Miguel, P.S. Sahni, in: C. Domb, J.L. Lebowitz (Eds.), Phase Transitions and Critical Phenomena, Vol. 8, Academic Press, New York, 1983.

[96] J.S. Langer, in: C. Godreche (Ed.), Solids Far from Equilibrium, Cambridge University Press, Cambridge, 1992.

[97] A.J. Bray, Adv. Phys. 43 (1994) 357.

[98] M. Abramowitz, I.S. Stegun (Eds.), Handbook of Mathematical Functions, Dover, New York, 1972.

[99] W. Spruijt, U. Ebert, W. van Saarloos, unpublished.

[100] C.M. Bender, S.A. Orszag, Advanced Mathematical Methods for Scientists and Engineers, McGraw-Hill, New York, 1978.

[101] L.A. Peletier, W.C. Troy, SIAM J. Math. Anal. 28 (1997) 1317.

[102] U. Ebert, W. van Saarloos, L.A. Peletier, Universal algebraic convergence in time of pulled fronts: the common mechanism for difference-differential and partial differential equations, Eur. J. Appl. Math., submitted for publication.

[103] O. Diekmann, J. Diff. Eqs. 33 (1979) 58.

[104] S. Fedotov, cond-mat/9807352.

[105] D. Potter, Computational Physics, Wiley, New York, 1973.

[106] D. Carpentier, private communication.

[107] X.Y. Wang. S. Fan, T. Kyu, Phys. Rev. E 56 (1997) R4931.

[108] P. Kaliappan, Physica D 11 (1984) 368.

[109] M. Otwinowski, R. Paul, W.G. Laidlaw, Phys. Lett. A 128 (1988) 483.

[110] G.S. Triantafyllou, K. Kupfer, A. Bers, Phys. Rev. Lett. 59 (1987) 1914.

[111] R.A.L. Jones, L.J. Norton, E.J. Kramer, F.S. Bates, P. Wiltzius, Phys. Rev. Lett. 66 (1991) 1326.

[112] U. Ebert, W. van Saarloos, C. Caroli, Phys. Rev. E 55 (1997) 1530.

[113] A. Torcini, S. Lepri, Phys. Rev. E 55 (1997) R3805.

[114] J.D. Logan, An Introduction to Nonlinear Partial Differential Equations, Wiley, New York, 1994.

[115] R.D. Benguria, M.C. Depassier, Phys. Rev. E 57 (1998) 6493.

[116] W. van Saarloos, P.C. Hohenberg, Physica D 69 (1993) 209 [Erratum to Physica D 56 (1992) 303]

[117] V.I. Arnold, Geometrical Methods in the Theory of Ordinary Differential Equations, Springer, New York, 1983.

[118] W.H. Press, B.P. Flannery, S.A. Teukolsky, W.T. Vetterling, Numerical Recipes, Cambridge University Press, Cambridge, 1986.

[119] D. Carpentier, P. Le Doussal, unpublished.

[120] P.E. Cladis, W. van Saarloos, in: Solitons in Liquid Crystals, Springer, Berlin, 1991. 